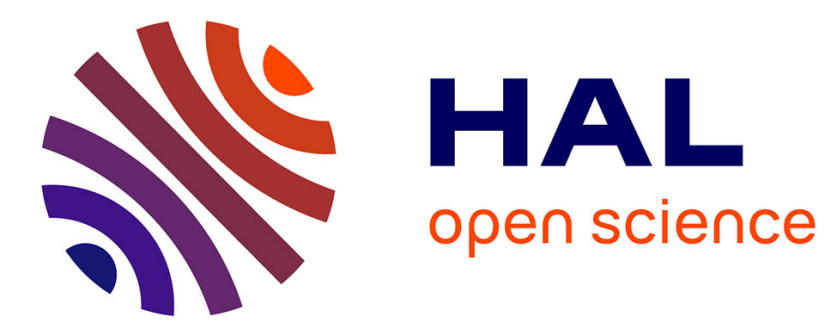

\title{
Lie Symmetries and CR Geometry
}

Joël Merker

\section{To cite this version:}

Joël Merker. Lie Symmetries and CR Geometry. Journal of Mathematical Sciences, 2008, 154 (6), pp.817-922. 10.1007/s10958-008-9201-5 . hal-03286232

\section{HAL Id: hal-03286232}

https:

\section{//hal-universite-paris-saclay.archives-ouvertes.fr/hal-03286232}

Submitted on 17 Jul 2021

HAL is a multi-disciplinary open access archive for the deposit and dissemination of scientific research documents, whether they are published or not. The documents may come from teaching and research institutions in France or abroad, or from public or private research centers.
L'archive ouverte pluridisciplinaire HAL, est destinée au dépôt et à la diffusion de documents scientifiques de niveau recherche, publiés ou non, émanant des établissements d'enseignement et de recherche français ou étrangers, des laboratoires publics ou privés. 


\title{
I: LIE SYMMETRIES OF PARTIAL DIFFERENTIAL EQUATIONS AND CR GEOMETRY
}

\author{
JOËL MERKER
}

Table of contents

1. Completely integrable systems of partial differential equations ....................

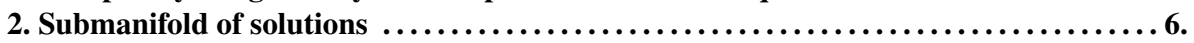

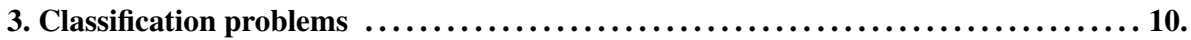

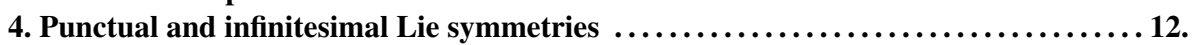

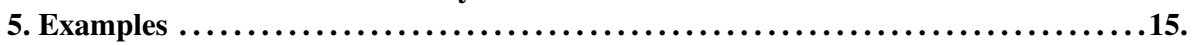

6. Transfer of Lie symmetries to the parameter space $\ldots \ldots \ldots \ldots \ldots \ldots \ldots \ldots \ldots \ldots \ldots . .18$.

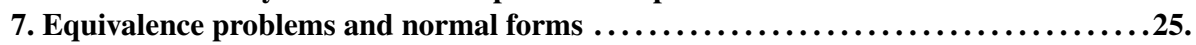

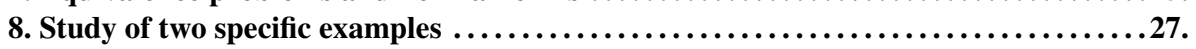

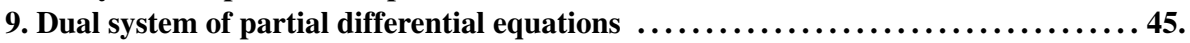

10. Fundamental pair of foliations and covering property $\ldots \ldots \ldots \ldots \ldots \ldots \ldots \ldots \ldots . \ldots . .46$.

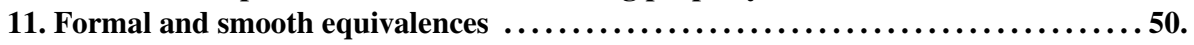

Journal of Mathematical Sciences (N. Y.), to appear

This memoir is divided in three parts ${ }^{1}$. Part I endeavours a general, new theory (inspired by modern CR geometry) of Lie symmetries of completely integrable PDE systems, viewed from their associated submanifold of solutions. Part II builds general combinatorial formulas for the prolongations of vector fields to jet spaces. Part III characterizes explicitly flatness of some systems of second order. The results presented here are original and did not appear in print elsewhere; most formulas of Parts II and III were checked by means of Maple Release 7.

\section{$\S 1$. COMPLETELY INTEGRABLE SYSTEMS OF PARTIAL DIFFERENTIAL EQUATIONS}

1.1. General systems. Let $\mathbb{K}=\mathbb{R}$ or $\mathbb{C}$. Let $n \in \mathbb{N}$ with $n \geqslant 1$ and let $x=\left(x^{1}, \ldots, x^{n}\right) \in$ $\mathbb{K}^{n}$. Also, let $m \in \mathbb{N}$ with $m \geqslant 1$ and let $y=\left(y^{1}, \ldots, y^{m}\right) \in \mathbb{K}^{m}$. For $\alpha \in \mathbb{N}^{n}$, we denote by a subscript $y_{x^{\alpha}}$ the partial derivative $\partial^{|\alpha|} y / \partial x^{\alpha}$ of a local map $\mathbb{K}^{n} \ni x \mapsto y(x) \in \mathbb{K}^{m}$.

Let $\kappa \in \mathbb{N}$ with $\kappa \geqslant 1$, let $p \in \mathbb{N}$ with $p \geqslant 1$, choose a collection of $p$ multiindices $\beta(1), \ldots, \beta(p) \in \mathbb{N}^{n}$ with $|\beta(q)| \geqslant 1$ for $q=1, \ldots, p$ and $\max _{1 \leqslant q \leqslant p}|\beta(q)|=\kappa$, and choose integers $j(1), \ldots, j(p)$ with $1 \leqslant j(q) \leqslant m$ for $q=1, \ldots, p$. In the present Part I, we study the Lie symmetries of a general system of analytic partial differential equations of the form:

$$
y_{x^{\alpha}}^{j}(x)=F_{\alpha}^{j}\left(x, y(x),\left(y_{x^{\beta(q)}}^{j(q)}(x)\right)_{1 \leqslant q \leqslant p}\right),
$$

where $j$ with $1 \leqslant j \leqslant m$ and $\alpha \in \mathbb{N}^{n}$ satisfy

$$
(j, \alpha) \neq(j, 0) \quad \text { and } \quad(j, \alpha) \neq(j(q), \beta(q)) .
$$

In particular, all $(\kappa+1)$-th partial derivatives of the unknown $y=y(x)$ depend on a certain precise set of derivatives of order $\leqslant \kappa$ : the system is complete. In addition, all the other partial derivatives of order $\leqslant \kappa$ do also depend on the same precise set of derivatives.

Here, we assume that $u=0$ is a local solution of the system $(\mathcal{E})$ and that the functions $F_{\alpha}^{j}$ are $\mathbb{K}$-algebraic (in the sense of Nash) or $\mathbb{K}$-analytic, in a neighborhood of the origin in

Date: 2011-5-27.

${ }^{1}$ Part II of [Me2005a] already appeared as [Me2005b]. 
$\mathbb{K}^{n+m+p}$. Even if our concern will be local throughout, we will not introduce any special notation to speak of open subsets and simply refer to various $\mathbb{K}^{\mu}$. We will study five concrete instances, the first three ones being classical.

Example 1.3. With $n=m=\kappa=1$, a second order ordinary differential equation

$$
y_{x x}=F\left(x, y, y_{x}\right)
$$

and more generally $y_{x^{\kappa+1}}=F\left(x, y, y_{x}, \ldots, y_{x^{\kappa}}\right)$, where $x, y \in \mathbb{K}$, see [Lie1883, EL1890, Tr1896, Ca1924, Se1931, Ca1932a, O11986, Ar1988, BK1989, GTW1989, HK1989, Ib1992, O11995, N2003].

Example 1.4. With $n \geqslant 2, m=1$ and $\kappa=1$, a complete system of second order equations

$$
y_{x^{i_{1}} x^{i_{2}}}=F_{i_{1}, i_{2}}\left(x^{i}, y, y_{x^{k}}\right), \quad 1 \leqslant i_{1}, i_{2} \leqslant n
$$

see [Ha1937, Ch1975, Su2001] and Part III below.

Example 1.5. Dually, with $n=1, m \geqslant 2$ and $\kappa=1$, an ordinary system of second order

$$
y_{x x}^{j}=F^{j}\left(x, y^{j_{1}}, y_{x}^{j_{1}}\right), \quad j=1, \ldots, m,
$$

see $[\mathrm{Fe} 1995, \mathrm{Me} 2004]$ and the references therein.

Example 1.6. With $n=1, m=2$ and $\kappa=1$, a system of the form

$$
\left\{\begin{array}{c}
y_{x}^{2}=F\left(x, y^{1}, y^{2}, y_{x}^{1}\right) \\
y_{x x}^{1}=G\left(x, y^{1}, y^{2}, y_{x}^{1}\right) .
\end{array}\right.
$$

Differentiating the first equation with respect to $x$ and substituting, we get the missing equation:

$$
\begin{aligned}
y_{x x}^{2} & =F_{x}+y_{x}^{1} F_{y^{1}}+y_{x}^{2} F_{y^{2}}+y_{x x}^{1} F_{y_{x}^{1}} \\
& =F_{x}+y_{x}^{1} F_{y^{1}}+y_{x}^{2} F_{y^{2}}+G F_{y_{x}^{1}} \\
& =: H\left(x, y^{1}, y^{2}, y_{x}^{1}\right) .
\end{aligned}
$$

Example 1.8. With $n=2, m=1$ and $\kappa=2$, a system of the form

$$
\left\{\begin{aligned}
y_{x^{2}} & =F\left(x^{1}, x^{2}, y, y_{x^{1}}, y_{x^{1} x^{1}}\right) \\
y_{x^{1} x^{1} x^{1}} & =G\left(x^{1}, x^{2}, y, y_{x^{1}}, y_{x^{1} x^{1}}\right) .
\end{aligned}\right.
$$

Here, five equations are missing. Differentiating the first equation with respect to $x^{1}$ and substituting:

$$
\begin{aligned}
y_{x^{1} x^{2}} & =F_{x^{1}}+y_{x^{1}} F_{y}+y_{x^{1} x^{1}} F_{y_{x^{1}}}+y_{x^{1} x^{1} x^{1}} F_{y_{x^{1} x^{1}}} \\
& =F_{x^{1}}+y_{x^{1}} F_{y}+y_{x^{1} x^{1}} F_{y_{x^{1}}}+G F_{y_{x^{1} x^{1}}} \\
& =: H\left(x^{1}, x^{2}, y, y_{x^{1}}, y_{x^{1} x^{1}}\right),
\end{aligned}
$$

and then similarly for $y_{x^{2} x^{2}}, y_{x^{1} x^{1} x^{2}}, y_{x^{1} x^{2} x^{2}}, y_{x^{2} x^{2} x^{2}}$.

1.10. Finitely nondegenerate generic submanifolds of $\mathbb{C}^{n+m}$. Examples $1.3,1.4,1.6$ and 1.8 (but not 1.5) are intrinsically linked to real submanifolds of complex submanifolds.

Let $M$ be a real algebraic or analytic local generic $\mathrm{CR}^{2}$ submanifold of $\mathbb{C}^{n+m}$ of codimension $m \geqslant 1$ and of CR dimension $n \geqslant 1$, and let $p \in M$. Classically, there exists local

\footnotetext{
${ }^{2}$ Fundamentals about Cauchy-Riemann geometry may be found in [Bo1991, BER1999, Me2005a, Me2005b, MP2005].
} 
holomorphic coordinates $t=(z, w) \in \mathbb{C}^{n} \times \mathbb{C}^{m}$ centered at $p$ in which $M$ is represented by

$$
w^{j}=\bar{\Theta}^{j}(z, \bar{z}, \bar{w}), \quad j=1, \ldots, m,
$$

for some local $\mathbb{C}$-analytic map $\Theta=\left(\Theta^{1}, \ldots, \Theta^{m}\right)$ satisfying the identity

$$
w \equiv \bar{\Theta}(z, \bar{z}, \Theta(\bar{z}, z, w))
$$

reflecting the fact that $M$ is real.

Definition 1.13. ([BER1999, Me2005a, Me2005b, MP2005]) $M$ is finitely nondegenerate if there exists an integer $\kappa \geqslant 1$ such that the local holomorphic map

$$
(\bar{z}, \bar{w}) \longmapsto\left(\bar{\Theta}_{z^{\beta}}^{j}(0, \bar{z}, \bar{w})\right)_{|\beta| \leqslant \kappa}^{1 \leqslant j \leqslant m}
$$

is of rank $n+m$ at $(\bar{z}, \bar{w})=(0,0)$.

From (1.12), the map $\bar{w} \mapsto \bar{\Theta}(0,0, \bar{w})$ is already of rank $m$ at $\bar{w}=0$. One then verifies ([BER1999, Me2005a, Me2005b, MP2005]) that there exist multiindices $\beta(1), \ldots, \beta(n) \in \mathbb{N}^{n}$ with $|\beta(k)| \geqslant 1$ for $k=1, \ldots, n$ and $\max _{1 \leqslant k \leqslant n}|\beta(k)|=\kappa$ together with integers $j(1), \ldots, j(n)$ with $1 \leqslant j(k) \leqslant m$ such that the local holomorphic map

$$
\mathbb{C}^{n+m} \ni(\bar{z}, \bar{w}) \longmapsto\left(\left(\bar{\Theta}^{j}(0, \bar{z}, \bar{w})\right)^{1 \leqslant j \leqslant m},\left(\bar{\Theta}_{z^{\beta(k)}}^{j(k)}(0, \bar{z}, \bar{w})\right)_{1 \leqslant k \leqslant n}\right) \in \mathbb{C}^{m+n}
$$

is of rank $n+m$ at $(\bar{z}, \bar{w})=(0,0)$.

1.16. Associated system of partial differential equations. Generalizing an idea which goes back to B. Segre in [Se1931, Se1932] $(n=m=1)$, applied by É. Cartan in [Ca1932a] and studied more recently in [Su2001, GM2003a], we may associate to $M$ a system of partial differential equations of the form $(\mathcal{E})$ as follows. Complexifying the variables $\bar{z}$ and $\bar{w}$, we introduce new independent variables $\zeta \in \mathbb{C}^{n}$ and $\xi \in \mathbb{C}^{m}$ together with the complex algebraic or analytic $m$-codimensional submanifold $\mathcal{M}$ of $\mathbb{C}^{2(n+m)}$ defined by

$$
w^{j}=\bar{\Theta}^{j}(z, \zeta, \xi), \quad j=1, \ldots, m .
$$

We then consider the "dependent variables" $w^{j}$ as algebraic or analytic functions of the "independent variables" $z^{k}$, with additional dependence on the extra "parameters" $(\zeta, \xi)$. Then by applying the differentiation $\partial^{|\alpha|} / \partial z^{\alpha}$ to (1.17), we get $w_{z^{\alpha}}^{j}(z)=\bar{\Theta}_{z^{\alpha}}^{j}(z, \zeta, \xi)$. Assuming finite nondegeneracy and writing these equations for $(j, \alpha)=(j(k), \beta(k))$, we obtain a system of $m+n$ equations:

$$
\left\{\begin{aligned}
w^{j}(z) & =\bar{\Theta}^{j}(z, \zeta, \xi), \quad j=1, \ldots, m, \\
w_{z^{\beta(k)}}^{j(k)}(z) & =\bar{\Theta}_{z^{\beta(k)}}^{j(k)}(z, \zeta, \xi), \quad k=1, \ldots, n .
\end{aligned}\right.
$$

By means of the implicit function theorem we can solve:

$$
(\zeta, \xi)=R\left(z^{k}, w^{j}(z), w_{z^{\beta(k)}}^{j(k)}(z)\right) .
$$

Finally, for every pair $(j, \alpha)$ different from $(j, 0)$ and from $(j(k), \beta(k))$, we may replace $(\zeta, \xi)$ by $R$ in the differentiated expression $w_{z^{\alpha}}^{j}(z)=\bar{\Theta}_{z^{\alpha}}^{j}(z, \zeta, \xi)$, which yields

$$
\begin{aligned}
w_{z^{\alpha}}^{j}(z) & =\bar{\Theta}_{z^{\alpha}}^{j}\left(z, R\left(z^{k}, w^{j}(z), w_{z^{\beta(k)}}^{j(k)}(z)\right)\right) \\
& =: F_{\alpha}^{j}\left(z^{k}, w^{j}(z), w_{z^{\beta(k)}}^{j(k)}(z)\right) .
\end{aligned}
$$

This is the system of partial differential equations associated to $M$. 
Example 1.21. (Continued) With $n=m=1$, i.e. $M \subset \mathbb{C}^{2}$ and $\kappa=1$, i.e. $M$ is Levi nondegenerate of equation

$$
w=\bar{w}+i z \bar{z}+\mathrm{O}_{3}
$$

where $z, \bar{z}$ are assigned weight 1 and $w, \bar{w}$ weight 2, B. Segre [Se1931] obtained $w_{z z}=$ $F\left(z, w, w_{z}\right)$. J. Faran [Fa1980] found some examples of such equations that cannot come from a $M \subset \mathbb{C}^{2}$. But the following was left unsolved.

Open problem 1.23. Characterize equations $y_{x x}=F\left(x, y, y_{x}\right)$ associated to a real analytic, Levi nondegenerate (i.e. $\kappa=1$ ) hypersurface $M \subset \mathbb{C}^{2}$. Can on read the reality condition (1.12) on $F$ ? In case of success, generalize to arbitrary $M \subset \mathbb{C}^{n+m}$.

Example 1.24. (Continued) Similarly, the system $\left(\mathcal{E}_{2}\right)$ comes from a Levi nondegenerate hypersurface $M \subset \mathbb{C}^{n+1}$ ([Ha1937, CM1974, Ch1975, Su2001]. Exercise: why $\left(\mathcal{E}_{3}\right)$ cannot come from any $M \subset \mathbb{C}^{\nu}$ ?

Example 1.25. (Continued) With $n=1, m=2$ and $\kappa=1$, the system $\left(\mathcal{E}_{4}\right)$ comes from a $M \subset \mathbb{C}^{3}$ which is Levi nondegenerate and satisfies

$$
T^{c} M+\left[T^{c} M, T^{c} M\right]+\left[T^{c} M,\left[T^{c} M, T^{c} M\right]\right]=T M
$$

at the origin, namely which has equations of the following form, after some elementary transformations ([Be1997, BES2005]):

$$
\begin{aligned}
& w^{1}=\bar{w}^{1}+i z \bar{z}+\mathrm{O}_{4}, \\
& w^{2}=\bar{w}^{2}+i z \bar{z}(z+\bar{z})+\mathrm{O}_{4},
\end{aligned}
$$

where $z, \bar{z}$ are assigned weight 1 and $w^{1}, w^{2}, \bar{w}^{1}, \bar{w}^{2}$ weight 2 .

Example 1.28. (Continued) With $n=2, m=1$ and $\kappa=2$, the system $\left(\mathcal{E}_{5}\right)$ comes from a hypersurface $M \subset \mathbb{C}^{3}$ of equation ([Eb1998, GM2003b, FK2005a, FK2005b, Eb2006, GM2006]):

$$
w=\bar{w}+i \frac{2 z^{1} \bar{z}^{1}+z^{1} z^{1} \bar{z}^{2}+\bar{z}^{1} \bar{z}^{1} z^{2}}{1-z^{2} \bar{z}^{2}}+\mathrm{O}_{4},
$$

where $z_{1}, \bar{z}_{1}, z_{2}, \bar{z}_{2}$ are assigned weight 1 and $w, \bar{w}$ weight 2 , with the assumption that the Levi form has rank exactly one at every point, and with the assumption that $M$ is 2-nondegenerate at 0 .

1.30. Jet spaces, contact forms and Frobenius integrability. Throughout the present Part I, we assume that the system $(\mathcal{E})$ is completely integrable, namely that the Pfaffian system naturally associated to $(\mathcal{E})$ in the appropriate jet space is involutive in the sense of Frobenius. This holds automatically in case $(\mathcal{E})$ comes from a generic submanifold $M \subset \mathbb{C}^{n+m}$. In general, we will construct a submanifold of solutions associated to $(\mathcal{E})$. So, we must explain complete integrability.

We denote by $\mathcal{J}_{n, m}^{\kappa}$ the space of $\kappa$-th jets of maps $\mathbb{K}^{n} \ni x \mapsto y(x) \in \mathbb{K}^{m}$. Let

$$
\left(x^{i}, y^{j}, y_{i_{1}}^{j}, y_{i_{1}, i_{2}}^{j}, \ldots \ldots, y_{i_{1}, i_{2}, \ldots, i_{\kappa}}^{j}\right) \in \mathbb{K}^{n+m+m n+m n^{2}+\cdots+m n^{\kappa}},
$$

denote the natural coordinates on $\mathcal{J}_{n, m}^{\kappa} \simeq \mathbb{K}^{n+m\left(1+n+\cdots+n^{\kappa}\right)}$. For instance, $\left(x, y, y_{1}\right) \in$ $\mathcal{J}_{1,1}^{1}$. We shall sometimes write them shortly:

$$
\left(x^{i}, y^{j}, y_{\beta}^{j}\right) \in \mathbb{K}^{n+m+m\left(n+\cdots+n^{\kappa}\right)},
$$

where $\beta \in \mathbb{N}^{n}$ varies and satisfies $|\beta| \leqslant \kappa$. Sometimes also, we consider these jet coordinates only up to their symmetries $y_{i_{1}, i_{2}, \ldots, i_{\lambda}}^{j}=y_{i_{\sigma(1)}, i_{\sigma(2)}, \ldots, i_{\sigma(\lambda)}}$, where $\sigma$ is a permutation

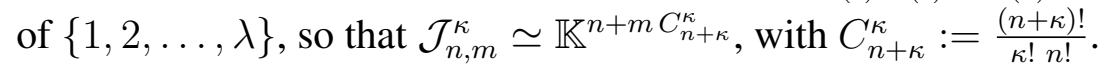


Having these notations at hand, we may develope the canonical system of contact forms on $\mathcal{J}_{n, m}^{\kappa}([\mathrm{O} 11995]$, [Stk2000]):

$$
\left\{\begin{aligned}
\theta^{j} & :=d y^{j}-\sum_{k=1}^{n} y_{k}^{j} d x^{k}, \\
\theta_{i_{1}}^{j} & :=d y_{i_{1}}^{j}-\sum_{k=1}^{n} y_{i_{1}, k}^{j} d x^{k} \\
\ldots \ldots \ldots \ldots \ldots \ldots \ldots \ldots \ldots \ldots \ldots \ldots \ldots \ldots \ldots, \ldots \ldots, i_{\kappa-1}, k & \ldots \ldots
\end{aligned}\right.
$$

For instance, with $n=m=1$ and $\kappa=2$, we have $\theta^{1}=d y-y_{1} d x$ and $\theta_{1}^{1}=d y_{1}-y_{2} d x$. These (linearly independent) one-forms generate a subspace $\mathcal{C} \mathcal{T}_{n, m}^{\kappa}$ of the cotangent $T^{*} \mathcal{J}_{n, m}^{\kappa}$ whose dimension equals $m C_{n+\kappa-1}^{\kappa-1}$. For the duality between forms and vectors, the orthogonal $\left(\mathcal{C} \mathcal{T}_{n, m}^{\kappa}\right)^{\perp}$ in $T \mathcal{J}_{n, m}^{\kappa}$ is spanned by the $n+m C_{n+\kappa-1}^{\kappa}$ vector fields:

$$
\left\{\begin{aligned}
D_{i} & :=\frac{\partial}{\partial x^{i}}+\sum_{j_{1}=1}^{m} y_{i}^{j_{1}} \frac{\partial}{\partial y^{j_{1}}}+\cdots+\sum_{j_{1}=1}^{m} \sum_{k_{1}, \ldots, k_{\kappa-1}=1}^{n} y_{i, k_{1}, \ldots, k_{\kappa-1}}^{j_{1}} \frac{\partial}{\partial y_{k_{1}, \ldots, k_{\kappa-1}}^{j_{1}}}, \\
T_{i_{1}, \ldots, i_{\kappa}}^{j_{1}} & :=\frac{\partial}{\partial y_{i_{1}, \ldots, i_{\kappa}}^{j_{1}}},
\end{aligned}\right.
$$

the first $n$ ones being the total differentiation operators, considered in Part II. For $n=$ $m=1, \kappa=2$, we get $\frac{\partial}{\partial x}+y_{1} \frac{\partial}{\partial y}+y_{2} \frac{\partial}{\partial y_{1}}$ and $\frac{\partial}{\partial y_{2}}$.

Classically ([O11986, BK1989, Ol1995]), one associates to $(\mathcal{E})$ its skeleton $\Delta_{\mathcal{E}}$, namely the $(n+m+p)$-dimensional submanifold of $\mathcal{J}_{n, m}^{\kappa+1}$ simply defined by the graphed equations:

$$
y_{\alpha}^{j}=F_{\alpha}^{j}\left(x, y,\left(y_{\beta(q)}^{j(q)}\right)_{1 \leqslant q \leqslant p}\right),
$$

for $(j, \alpha) \neq(j, 0)$ and $\neq(j(q), \beta(q))$ with $|\alpha| \leqslant \kappa+1$. Clearly, the natural coordinates on $\Delta_{\mathcal{E}}$ are:

$$
\left(x, y,\left(y_{\beta(q)}^{j(q)}\right)_{1 \leqslant q \leqslant p}\right) \equiv\left(x, y,\left(y_{l_{1}(q), \ldots, l_{\lambda_{q}}(q)}^{j(q)}\right)_{1 \leqslant q \leqslant p}\right) \in \mathbb{K}^{n} \times \mathbb{K}^{m} \times \mathbb{K}^{p},
$$

where $\lambda_{q}:=|\beta(q)|$ and $\left(l_{1}(q), \ldots, l_{\lambda_{q}}(q)\right):=\beta(q)$.

Next, in view of the form (1.34) of the generators of $\left(\mathcal{C} \mathcal{T}_{n, m}^{\kappa+1}\right)^{\perp}$ and in view of the equations of $\Delta_{\mathcal{E}}$, the intersection

$$
\left(\mathcal{C} \mathcal{T}_{n, m}^{\kappa+1}\right)^{\perp} \cap T \Delta_{\mathcal{E}}
$$

is a vector subbundle of $T \Delta_{\mathcal{E}}$ that is generated by $n$ linearly independent vector fields obtained by restricting the $D_{i}$ to $\Delta_{\mathcal{E}}$, which yields:

$$
\left\{\begin{aligned}
\mathrm{D}_{i}=\frac{\partial}{\partial x^{i}}+ & \sum_{j=1}^{m} \mathbf{A}_{i}^{j}\left(x^{i_{1}}, y^{j_{1}}, y_{\beta\left(q_{1}\right)}^{j\left(q_{1}\right)}\right) \frac{\partial}{\partial y^{j}}+ \\
& +\sum_{q=1}^{p} \mathbf{B}_{i}^{q}\left(x^{i_{1}}, y^{j_{1}}, y_{\beta\left(q_{1}\right)}^{j\left(q_{1}\right)}\right) \frac{\partial}{\partial y_{\beta(q)}^{j(q)}}
\end{aligned}\right.
$$


$i=1, \ldots, n$, where the coefficients $\mathbf{A}_{i}^{j}$ and $\mathbf{B}_{i}^{q}$ are given by:

$$
\begin{aligned}
& \mathbf{A}_{i}^{j}:=\left\{\begin{array}{l}
y_{i}^{j} \text { if the variable } y_{i}^{j} \text { appears among the } p \text { variables } y_{\beta\left(q_{1}\right)}^{j\left(q_{1}\right)} \\
F_{i}^{j} \text { otherwise } ;
\end{array}\right. \\
& \mathbf{B}_{i}^{q}:=\left\{\begin{array}{l}
y_{i, l_{1}(q), \ldots, l_{\lambda_{q}}(q)}^{j(q)} \text { if } y_{l_{1}(q), \ldots, l_{\lambda_{q}(q)}^{j(q)}}^{j(q)} \\
F_{i, l_{1}(q), \ldots, l_{\lambda_{q}}(q)}^{j\left(q_{1}\right.} \text { otherwise. }
\end{array}\right.
\end{aligned}
$$

Example 1.40. For $\left(\mathcal{E}_{1}\right)$, we get $\mathrm{D}=\frac{\partial}{\partial x}+y_{1} \frac{\partial}{\partial x}+F\left(x, y, y_{1}\right) \frac{\partial}{\partial y_{2}}$; exercise: treat $\left(\mathcal{E}_{2}\right)$ and $\left(\mathcal{E}_{3}\right)$. For $\left(\mathcal{E}_{4}\right)$, we get $\mathrm{D}=\frac{\partial}{\partial x}+y_{1}^{1} \frac{\partial}{\partial y^{1}}+F \frac{\partial}{\partial y^{2}}+G \frac{\partial}{\partial y_{1}^{1}}$. For $\left(\mathcal{E}_{5}\right)$, whose skeleton is written $y_{2}=F, y_{1,1,1}=G, y_{1,2}=H, y_{1,1,2}=K$, with $F, G, H, K$ being functions of $\left(x^{1}, x^{2}, y, y_{1}, y_{1,1}\right)$, we get

$$
\begin{aligned}
& \mathrm{D}_{1}=\frac{\partial}{\partial x^{1}}+y_{1} \frac{\partial}{\partial y}+y_{1,1} \frac{\partial}{\partial y_{1}}+G \frac{\partial}{\partial y_{1,1}}, \\
& \mathrm{D}_{2}=\frac{\partial}{\partial x^{2}}+F \frac{\partial}{\partial y}+H \frac{\partial}{\partial y_{1}}+K \frac{\partial}{\partial y_{1,1}} .
\end{aligned}
$$

Definition 1.42. The system $(\mathcal{E})$ is completely integrable if the $n$ vector fields (1.38) satisfy the Frobenius integrability condition, namely every Lie bracket $\left[\mathrm{D}_{i_{1}}, \mathrm{D}_{i_{2}}\right], 1 \leqslant$ $i_{1}, i_{2} \leqslant n$, is a linear combination of the vector fields $\mathrm{D}_{1}, \ldots, \mathrm{D}_{n}$.

Because of their specific form (1.38), we must then have in fact $\left[D_{i_{1}}, D_{i_{2}}\right]=0$. For $n=1$, the condition is of course void.

\section{$\S 2$. SUBMANIFOLD OF SOLUTIONS}

2.1. Fundamental foliation of the skeleton. As the vector fields $D_{i}$ commute, they equip the skeleton $\Delta_{\mathcal{E}} \simeq \mathbb{K}^{n+m+p}$ with a foliation $\mathrm{F}_{\Delta_{\mathcal{E}}}$ by $n$-dimensional integral manifolds which are (approximately) directed along the $x$-axis. We draw a diagram (see only the left side).

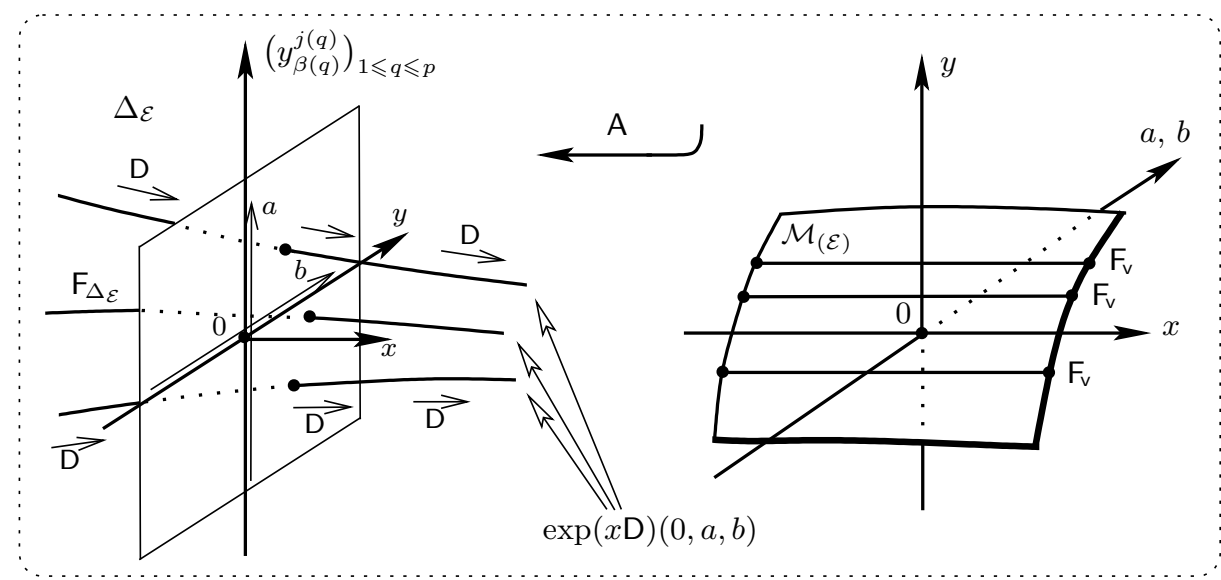

The (abstract, not numerical) integration of $(\mathcal{E})$ is thus straightforwardly completed: the set of solutions coincides with the set of leaves of $F_{\Delta_{\mathcal{E}}}$. This is the true geometric content, viewed in the appropriate jet space, of the assumption of complete integrability. 
2.2. General solution and submanifold of solutions. To construct the submanifold of solutions $\mathcal{M}_{(\mathcal{E})}$ associated to $(\mathcal{E})$ (sketched in the right hand side), we execute some elementary analytico-geometric constructions.

At first, we duplicate the coordinates $\left(y_{\beta(q)}^{j(q)}, y^{j}\right) \in \mathbb{K}^{p} \times \mathbb{K}^{m}$ by introducing a new subspace of coordinates $(a, b) \in \mathbb{K}^{p} \times \mathbb{K}^{m}$; thus, on the left diagram, we draw a vertical plane together with $a$ - and $b$-axes. The leaves of the foliation $\mathrm{F}_{\Delta_{\mathcal{E}}}$ are uniquely determined by their intersections with this plane, consisting of points of coordinates $(0, a, b) \in \mathbb{K}^{n} \times$ $\mathbb{K}^{p} \times \mathbb{K}^{m}$.

Such points $(0, a, b)$ correspond to the initial conditions $\left(y_{x^{\beta(q)}}^{j(q)}(0), y(0)\right)$ for the general solution of $(\mathcal{E})$. In fact, the (concatenated, multiple) flow of $\left\{D_{1}, \ldots, D_{n}\right\}$ is given by (2.3)

$\exp \left(x^{n} \mathrm{D}_{n}\left(\cdots\left(\exp \left(x^{1} \mathrm{D}_{1}(0, a, b)\right)\right) \cdots\right)\right)=(x, \Pi(x, a, b), \Omega(x, a, b)) \in \mathbb{K}^{n} \times \mathbb{K}^{m} \times \mathbb{K}^{p}$, for some two local analytic maps $\Pi=\left(\Pi^{1}, \ldots, \Pi^{m}\right)$ and $\Omega=\left(\Omega^{1}, \ldots, \Omega^{p}\right)$ and the next lemma is straightforward.

Lemma 2.4. The general solution of $(\mathcal{E})$ is

$$
y(x):=\Pi(x, a, b),
$$

where $(a, b)$ varies in $\mathbb{K}^{p} \times \mathbb{K}^{m}$. Furthermore, for $q=1, \ldots, p$ :

$$
\Omega^{q}(x, a, b) \equiv \Pi_{x^{\beta(q)}}^{j(q)}(x, a, b) .
$$

This leads to introducing a fundamental geometric object.

Definition 2.7. The submanifold of solutions $\mathcal{V}_{\mathcal{S}}(\mathcal{E})$ associated to $(\mathcal{E})$ is the analytic submanifold of $\mathbb{K}_{x}^{n} \times \mathbb{K}_{y}^{m} \times \mathbb{K}_{a}^{p} \times \mathbb{K}_{b}^{m}$ defined by the Cartesian equations:

$$
0=-y^{j}+\Pi^{j}(x, a, b), \quad j=1, \ldots, m .
$$

There is a strong interplay between the study of $(\mathcal{E})$ and the geometry of $\mathcal{V}_{\mathcal{S}}(\mathcal{E})$. By construction, the diffeomorphism:

$$
\left\{\begin{array}{l}
\mathrm{A}: \mathbb{K}^{n+p+m}\left[\text { coordinates }\left(x^{i}, a^{q}, b^{j}\right)\right] \longrightarrow \mathbb{K}^{n+m+p}\left[\operatorname{coordinates}\left(x^{i}, y^{j}, y_{\beta(q)}^{j(q)}\right)\right] \\
\mathrm{A}\left(x^{i}, a^{q}, b^{j}\right):=\left(x^{i}, \Pi^{j}(x, a, b), \Pi_{x^{\beta(q)}}^{j(q)}(x, a, b),\right)
\end{array}\right.
$$

sends the foliation $\mathrm{F}_{\mathrm{v}}$ by the variables $x$ whose leaves are $\{a=$ cst., $b=$ cst. $\}$ (see the diagram), to the previous foliation $\mathrm{F}_{\Delta_{\mathcal{E}}}$.

2.10. PDE system associated to a submanifold. Inversely, let $\mathcal{M}$ be a submanifold of $\mathbb{K}_{x}^{n} \times \mathbb{K}_{y}^{m} \times \mathbb{K}_{a}^{p} \times \mathbb{K}_{b}^{m}$ of the form

$$
y^{j}=\Pi^{j}(x, a, b), \quad j=1, \ldots, m .
$$

A necessary condition for it to be the complexification of a generic $M \subset \mathbb{C}^{n+m}$ is that $p=n$ (answer to an exercise above).

Definition 2.12. $\mathcal{M}$ is solvable with respect to the parameters if $b \mapsto \Pi(0,0, b)$ of rank $m$ at $b=0$ and if there exist $\kappa \geqslant 1$, multiindices $\beta(1), \ldots, \beta(p) \in \mathbb{N}^{n}$ with $|\beta(q)| \geqslant 1$ for $q=1, \ldots, p$ and $\max _{1 \leqslant q \leqslant p}|\beta(q)|=\kappa$, together with integers $j(1), \ldots, j(p)$ with $1 \leqslant j(q) \leqslant m$ such that the local $\mathbb{K}$-analytic map

$$
\mathbb{K}^{m+p} \ni(a, b) \longmapsto\left(\left(\Pi^{j}(0, a, b)\right)^{1 \leqslant j \leqslant m},\left(\Pi_{x^{\beta(q)}}^{j(q)}(0, a, b)\right)_{1 \leqslant q \leqslant p}\right) \in \mathbb{K}^{m+p}
$$

is of rank equal to $m+p$ at $(a, b)=(0,0)$ 
When $\mathcal{M}$ is the submanifold of solutions of a system $(\mathcal{E})$, it is automatically solvable with respect to the variables, the pairs $(j(q), \beta(q))$ being the same as in the arguments of the right hand sides $F_{\alpha}^{j}$ in $(\mathcal{E})$. Proceeding as in $\S 1.16$, we may associate to $\mathcal{M}$ a system of the form $(\mathcal{E})$. Since we need introduce some new notation, let us repeat the argument.

Considering $y=y(x)=\Pi(x, a, b)$ as a function of $x$ with extra parameters $(a, b)$ and applying $\partial^{|\alpha|} / \partial x^{\alpha}$, we get $y_{x^{\alpha}}^{j}(x)=\Pi_{x^{\alpha}}^{j}(x, a, b)$. Writing only the relevant $(m+p)$ equations:

$$
\left\{\begin{array}{l}
y^{j}(x)=\Pi^{j}(x, a, b), \\
y_{x^{\beta(q)}}^{j(q)}=\Pi_{x^{\beta(q)}}^{j(q)}(x, a, b),
\end{array}\right.
$$

the assumption of solvability with respect to parameters enables to get

$$
\left\{\begin{array}{l}
a^{q}=A^{q}\left(x^{i}, y^{j}, y_{\beta\left(q_{1}\right)}^{j\left(q_{1}\right)}\right) \\
b^{j}=B^{j}\left(x^{i}, y^{j_{1}}, y_{\beta(q)}^{j(q)}\right) .
\end{array}\right.
$$

For every $(j, \alpha) \neq(j, 0)$ and $\neq(j(q), \beta(q))$, we then replace $(a, b)$ in $y_{x^{\alpha}}^{j}=\Pi_{x^{\alpha}}^{j}$ :

$$
\begin{aligned}
y_{x^{\alpha}}^{j}(x) & =\Pi_{x^{\alpha}}^{j}\left(x, A\left(x^{i}, y^{j_{1}}(x), y_{\beta(q)}^{j(q)}(x)\right), B\left(x^{i}, y^{j_{1}}(x), y_{\beta(q)}^{j(q)}(x)\right)\right) \\
& =: F_{\alpha}^{j}\left(x^{i}, y^{j_{1}}(x), y_{x^{\beta(q)}}^{j(q)}(x)\right) .
\end{aligned}
$$

Proposition 2.17. There is a one-to-one correspondence

$$
\left(\mathcal{E}_{\mathcal{M}}\right)=(\mathcal{E}) \longleftrightarrow \mathcal{M}=\mathcal{M}_{(\mathcal{E})},
$$

between completely integrable systems of partial differential equations of the general form $(\mathcal{E})$ and submanifolds (of solutions) $\mathcal{M}$ of the form (2.11) which are solvable with respect to the parameters. Of course

$$
\left(\mathcal{E}_{\mathcal{M}_{(\mathcal{E})}}\right)=(\mathcal{E}) \quad \text { and } \quad \mathcal{M}_{\left(\mathcal{E}_{\mathcal{M}}\right)}=\mathcal{M}
$$

2.20. Transfer of total differentiations. We notice that the auxiliary functions $A^{q}$ and $B^{j}$ enable to express the inverse of A:

$$
\mathrm{A}^{-1}:\left(x^{i_{1}}, y^{j_{1}}, y_{\beta\left(q_{1}\right)}^{j\left(q_{1}\right)}\right) \longmapsto\left(x^{i}, A^{q}\left(x^{i_{1}}, y^{j_{1}}, y_{\beta\left(q_{1}\right)}^{j\left(q_{1}\right)}\right), B^{j}\left(x^{i_{1}}, y^{j_{1}}, y_{\beta\left(q_{1}\right)}^{j\left(q_{1}\right)}\right)\right) .
$$

More importantly, the total differentiation operator considerably simplifies when viewed on $\mathcal{M}$. This observation is useful for translating differential invariants of $(\mathcal{E})$ as differential invariants of $\mathcal{M}$.

Lemma 2.22. Through A, for $i=1, \ldots, n$, the pull-back of the total differentiation operator $\mathrm{D}_{i}$ is simply $\frac{\partial}{\partial x^{i}}$, or equivalently:

$$
\mathrm{A}_{*}\left(\frac{\partial}{\partial x^{i}}\right)=\mathrm{D}_{i}
$$

Proof. Let $\ell=\ell\left(x^{i}, y^{j}, y_{\beta(q)}^{j(q)}\right)$ be any function defined on $\Delta_{\mathcal{E}}$. Composing with A yields the function $\Lambda:=\ell \circ$ A, i.e.

$$
\Lambda(x, a, b) \equiv \ell\left(x^{i}, \Pi^{j}(x, a, b), \Pi_{x^{\beta(q)}}^{j(q)}(x, a, b)\right) .
$$

Differentiating with respect to $x^{i}$, we get, dropping the arguments:

$$
\frac{\partial \Lambda}{\partial x^{i}}=\frac{\partial \ell}{\partial x^{i}}+\sum_{j=1}^{m} \Pi_{x^{i}}^{j} \frac{\partial \ell}{\partial y^{j}}+\sum_{q=1}^{p} \Pi_{x^{i} x^{\beta(q)}}^{j(q)} \frac{\partial \ell}{\partial y_{x^{\beta(q)}}^{j(q)}} .
$$


Replacing the appearing $\Pi_{x^{\alpha}}^{j}$ for which $(j, \alpha) \neq(j, 0)$ and $\neq(j(q), \beta(q))$ by $F_{\alpha}^{j}$, we recover $\mathrm{D}_{i}$ as defined by $(1.38)$, whence $\frac{\partial \Lambda}{\partial x^{i}}=\mathrm{D}_{i} \ell$.

2.26. Transfer of algebrico-differential expressions. The diffeomorphism $A$ may be used to translate algebrico-differential expressions from $\mathcal{M}$ to $(\mathcal{E})$ and vice-versa:

$$
\mathrm{I}_{\mathcal{M}}\left(J_{x, a, b}^{\lambda+\kappa+1} \Pi\right) \longleftrightarrow \mathrm{I}_{(\mathcal{E})}\left(J_{x, y, y_{1}}^{\lambda} F\right) .
$$

Here, $\lambda \in \mathbb{N}$, the letter $J$ is used to denote jets, and $\mathrm{I}=\mathrm{I}_{\mathcal{M}}$ or $=\mathrm{I}_{(\mathcal{E})}$ is a polynomial or more generally, a quotient of polynomials with respect to its jet arguments. Notice the shift by $\kappa+1$ of the jet orders.

Example 2.28. Suppose $n=m=1$ and $\kappa=1$. Then $F=\Pi_{x x}$. As an exercise, let us compute $F_{x}, F_{y}, F_{y_{1}}$ in terms of $J_{x, a, b}^{3} \Pi$. We start with the identity

$$
F\left(x, y, y_{1}\right) \equiv \Pi_{x x}\left(x, A\left(x, y, y_{1}\right), B\left(x, y, y_{1}\right)\right),
$$

that we differentiate with respect to $x$, to $y$ and to $y_{1}$ :

$$
\begin{aligned}
& F_{x}=\Pi_{x x x}+\Pi_{x x a} A_{x}+\Pi_{x x b} B_{x}, \\
& F_{y}=\quad \Pi_{x x a} A_{y}+\Pi_{x x b} B_{y}, \\
& F_{y_{1}}=\quad \Pi_{x x a} A_{y_{1}}+\Pi_{x x b} B_{y_{1}} .
\end{aligned}
$$

Thus, we need to compute $A_{x}, A_{y}, A_{y_{1}}, B_{x}, B_{y}, B_{y_{1}}$. This is easy: it suffices to differentiate the two identities that define $A$ and $B$ as implicit functions, namely:

$$
\begin{aligned}
y & \equiv \Pi\left(x, A\left(x, y, y_{1}\right), B\left(x, y, y_{1}\right)\right) \quad \text { and } \\
y_{1} & \equiv \Pi_{x}\left(x, A\left(x, y, y_{1}\right), B\left(x, y, y_{1}\right)\right)
\end{aligned}
$$

with respect to $x$, to $y$ and to $y_{1}$, which gives six new identities:

$$
\begin{aligned}
& 0=\Pi_{x}+\Pi_{a} A_{x}+\Pi_{b} B_{x}, \quad 0=\Pi_{x x}+\Pi_{x a} A_{x}+\Pi_{x b} B_{x}, \\
& 1=\quad \Pi_{a} A_{y}+\Pi_{b} B_{y}, \quad 0=\quad \Pi_{x a} A_{y}+\Pi_{x b} B_{y} \\
& 0=\quad \Pi_{a} A_{y_{1}}+\Pi_{b} B_{y_{1}}, \quad 1=\quad \Pi_{x a} A_{y_{1}}+\Pi_{x b} B_{y_{1}},
\end{aligned}
$$

and to solve each of the three linear systems of two equations located in a line, noticing that their common determinant $\Pi_{b} \Pi_{x a}-\Pi_{a} \Pi_{x b}$ does not vanish at the origin, since $\Pi=$ $b+x a+\mathrm{O}_{3}$. By elementary Cramer formulas, we get:

$$
\left\{\begin{aligned}
A_{x} & =\frac{-\Pi_{b} \Pi_{x x}+\Pi_{x} \Pi_{x b}}{\Pi_{b} \Pi_{x a}-\Pi_{a} \Pi_{x b}}, & B_{x} & =\frac{-\Pi_{x} \Pi_{x a}+\Pi_{a} \Pi_{x x}}{\Pi_{b} \Pi_{x a}-\Pi_{a} \Pi_{x b}}, \\
A_{y} & =\frac{-\Pi_{x b}}{\Pi_{b} \Pi_{x a}-\Pi_{a} \Pi_{x b}}, & B_{y} & =\frac{\Pi_{x a}}{\Pi_{b} \Pi_{x a}-\Pi_{a} \Pi_{x b}}, \\
A_{y_{1}} & =\frac{\Pi_{b}}{\Pi_{b} \Pi_{x a}-\Pi_{a} \Pi_{x b}}, & B_{y_{1}} & =\frac{-\Pi_{a}}{\Pi_{b} \Pi_{x a}-\Pi_{a} \Pi_{x b}} .
\end{aligned}\right.
$$

Replacing in (2.30), no simplification occurs and we get what we wanted:

$$
\left\{\begin{array}{l}
F_{x}=\Pi_{x x x}+\frac{\Pi_{x x a}\left[-\Pi_{b} \Pi_{x x}+\Pi_{x} \Pi_{x b}\right]+\Pi_{x x b}\left[-\Pi_{x} \Pi_{x a}+\Pi_{a} \Pi_{x x}\right]}{\Pi_{b} \Pi_{x a}-\Pi_{a} \Pi_{x b}} \\
F_{y}=\frac{-\Pi_{x x a} \Pi_{x b}+\Pi_{x x b} \Pi_{x a}}{\Pi_{b} \Pi_{x a}-\Pi_{a} \Pi_{x b}} \\
F_{y_{1}}=\frac{\Pi_{x x a} \Pi_{b}-\Pi_{x x b} \Pi_{a}}{\Pi_{b} \Pi_{x a}-\Pi_{a} \Pi_{x b}}
\end{array}\right.
$$

One sees D $F=F_{x}+\Pi_{x} F_{y}+\Pi_{x x} F_{y_{1}}=\Pi_{x x x}$ simply, as predicted by Lemma 2.22.

Second order derivatives $F_{x x}, F_{x y}, F_{x y_{1}}, F_{y y}, F_{y y_{1}}, F_{y_{1} y_{1}}$ have still reasonable complexity, when expressed in terms of $J_{x, a, b}^{4} \Pi$. Beyond, the computations explode. 
Open question 2.35. A second order ordinary differential equation $y_{x x}=F\left(x, y, y_{x}\right)$ has two fundamental differential invariants, namely ([Tr1896, Ca1924, GTW1989, O11995]):

$$
\begin{aligned}
& \mathrm{I}_{\left(\mathcal{E}_{1}\right)}^{1}:=\frac{\partial^{4} F}{\partial y_{1}^{4}} \quad \text { and } \\
& \mathrm{I}_{\left(\mathcal{E}_{1}\right)}^{2}:=\mathrm{DD}\left(F_{y_{1} y_{1}}\right)-F_{y_{1}} \mathrm{D}\left(F_{y_{1} y_{1}}\right)-4 \mathrm{D}\left(F_{y y_{1}}\right)+6 F_{y y}-3 F_{y} F_{y_{1} y_{1}}+4 F_{y_{1}} F_{y y_{1}} .
\end{aligned}
$$

Compute $\mathrm{I}_{\mathcal{M}_{1}}^{1}$ and $\mathrm{I}_{\mathcal{M}_{1}}^{2}$.

Although the notion of diffeomorphism is clear and apparently obvious from the intuitive, geometric and conceptual viewpoints, in concrete applications and in explicit computations, it almost never straightforward to transfer algebrico-differential objects.

Open problem 2.37. For general $(\mathcal{E})$ and $\mathcal{M}$, build closed combinatorial formulas executing the double translation (2.27).

2.38. Plan for the sequel. We will endeavour a general theory showing that the study of systems $(\mathcal{E})$ and the study of submanifolds of solutions $\mathcal{M}$ gives complementary views on the same object. In fact, Lie symmetries, equivalence problems, Cartan connections, normal forms and classification lists may be endeavoured on both sides, yielding essentially equivalent results, though the translation is seldom straightforward. In Section 3, 4 and 5, we review some features from the side $(\mathcal{E})$, before studying some aspects from the side of $\mathcal{M}$. A more systematic and complete approach shall appear as a monography.

\section{$\S 3$. ClassificATION PROBLEMS}

3.1. Transformations of PDE systems. Through a local $\mathbb{K}$-analytic change of variables close to the identity $(x, y) \mapsto \varphi(x, y)=:\left(x^{\prime}, y^{\prime}\right)$, the system $(\mathcal{E})$ transforms to a similar system, with primes:

$$
y_{x^{\prime \alpha}}^{\prime j}\left(x^{\prime}\right)=F_{\alpha}^{\prime j}\left(x^{\prime}, y^{\prime}\left(x^{\prime}\right),\left(y_{x^{\prime \beta(q)}}^{\prime j(q)}\left(x^{\prime}\right)\right)_{1 \leqslant q \leqslant p}\right) .
$$

Example 3.2. Coming back temporarily to the notations of $\S 1.12($ II), with $n=m=$ $\kappa=1$, assume that $y_{x x}=f\left(x, y, y_{x}\right)$ transforms to $Y_{X X}=F\left(X, Y, Y_{X}\right)$ through a local diffeomorphism $(x, y) \mapsto(X, Y)=(X(x, y), Y(x, y))$. How $F$ is related to $f$ ? By symmetry, it suffices to compute $f$ in terms of $F, X, Y$. The prolongation to $\mathcal{J}_{1,1}^{2}$ of the diffeomorphism has components ([BK1989, Me2004]):

$$
Y_{X}=\frac{Y_{x}+y_{x} Y_{y}}{X_{x}+y_{x} X_{y}}
$$

and

$$
\begin{aligned}
& Y_{X X}=\frac{1}{\left[X_{x}+y_{x} X_{y}\right]^{3}}( y_{x x} \cdot\left|\begin{array}{cc}
X_{x} & X_{y} \\
Y_{x} & Y_{y}
\end{array}\right|+\left|\begin{array}{cc}
X_{x} & X_{x x} \\
Y_{x} & Y_{x x}
\end{array}\right|+ \\
&+y_{x} \cdot\left\{2\left|\begin{array}{cc}
X_{x} & X_{x y} \\
Y_{x} & Y_{x y}
\end{array}\right|-\left|\begin{array}{cc}
X_{x x} & X_{y} \\
Y_{x x} & Y_{y}
\end{array}\right|\right\}+ \\
&+y_{x} y_{x} \cdot\left\{\left|\begin{array}{cc}
X_{x} & X_{y y} \\
Y_{x} & Y_{y y}
\end{array}\right|-2\left|\begin{array}{cc}
X_{x y} & X_{y} \\
Y_{x y} & Y_{y}
\end{array}\right|\right\}+ \\
&\left.+y_{x} y_{x} y_{x} \cdot\left\{-\left|\begin{array}{cc}
X_{y y} & X_{y} \\
Y_{y y} & Y_{y}
\end{array}\right|\right\}\right) .
\end{aligned}
$$


It then suffices to replace $Y_{X X}$ above by $F\left(X, Y, Y_{X}\right)$ and to solve $y_{x x}$ :

$$
\begin{aligned}
y_{x x}=\frac{1}{\left|\begin{array}{cc}
X_{x} & X_{y} \\
Y_{x} & Y_{y}
\end{array}\right|}\left(\left[X_{x}+y_{x} X_{y}\right]^{3} F\left(X, Y, \frac{Y_{x}+y_{x} Y_{y}}{X_{x}+y_{x} X_{y}}\right)-\left|\begin{array}{cc}
X_{x} & X_{x x} \\
Y_{x} & Y_{x x}
\end{array}\right|+\right. \\
+y_{x} \cdot\left\{-2\left|\begin{array}{cc}
X_{x} & X_{x y} \\
Y_{x} & Y_{x y}
\end{array}\right|+\left|\begin{array}{cc}
X_{x x} & X_{y} \\
Y_{x x} & Y_{y}
\end{array}\right|\right\}+ \\
+y_{x} y_{x} \cdot\left\{-\left|\begin{array}{cc}
X_{x} & X_{y y} \\
Y_{x} & Y_{y y}
\end{array}\right|+2\left|\begin{array}{cc}
X_{x y} & X_{y} \\
Y_{x y} & Y_{y}
\end{array}\right|\right\}+ \\
\left.+y_{x} y_{x} y_{x} \cdot\left\{\begin{array}{cc}
X_{y y} & X_{y} \\
Y_{y y} & Y_{y}
\end{array} \mid\right\}\right) \\
=: f\left(x, y, y_{x}\right) .
\end{aligned}
$$

Open problem 3.6. Find general formulas expressing the $F_{\alpha}^{j}$ in terms of $F_{\alpha}^{\prime j}, x^{\prime i}, y^{\prime j}$.

Conversely, given two such systems $(\mathcal{E})$ and $\left(\mathcal{E}^{\prime}\right)$, when do they transform to each other? Let $\pi_{\kappa, p}^{\prime}$ denote the projection from $\mathcal{J}_{n, m}^{\prime \kappa+1}$ to $\Delta_{\mathcal{E}^{\prime}}$ defined by

$$
\pi_{\kappa, p}^{\prime}\left(x^{\prime i}, y^{\prime j}, y_{i_{1}}^{\prime j}, \ldots, y_{i_{1}, \ldots, i_{\kappa+1}}^{\prime j}\right):=\left(x^{\prime i}, y^{\prime j}, y_{\beta(q)}^{\prime j(q)}\right) .
$$

Let $\varphi^{(\kappa+1)}$ be the $(\kappa+1)$-th prolongation of $\varphi$ (Section 1(II)).

Lemma 3.8. ([O11986, BK1989, O11995]) The following three conditions are equivalent:

(1) $\varphi$ transforms $(\mathcal{E})$ to $\left(\mathcal{E}^{\prime}\right)$;

(2) its $(\kappa+1)$-th prolongation $\varphi^{(\kappa+1)}: \mathcal{J}_{n, m}^{\kappa+1} \rightarrow \mathcal{J}_{n, m}^{\prime \kappa+1}$ maps $\Delta_{\mathcal{E}}$ to $\Delta_{\mathcal{E}^{\prime}}$;

(3) $\varphi^{(\kappa+1)}: \mathcal{J}_{n, m}^{\kappa+1} \rightarrow \mathcal{J}_{n, m}^{\prime \kappa+1}$ maps $\Delta_{\mathcal{E}}$ to $\Delta_{\mathcal{E}^{\prime}}$ and the associated map

$$
\Phi_{\mathcal{E}, \mathcal{E}^{\prime}}:=\pi_{\kappa, p}^{\prime} \circ\left(\left.\varphi^{(\kappa+1)}\right|_{\Delta_{\mathcal{E}}}\right)
$$

sends every leaf of $\mathrm{F}_{\Delta_{\mathcal{E}}}$ to some leaf of $\mathrm{F}_{\Delta_{\mathcal{E}^{\prime}}}$.

Equivalence problem 3.10. Find an algorithm to decide whether two given $(\mathcal{E})$ and $\left(\mathcal{E}^{\prime}\right)$ are equivalent.

Élie Cartan's widely applicable method (not reviewed here; [Ca1937, Ste1983, G1989, HK1989, Fe1995, O11995]) provides an answer "in principle" to this question by reducing to an $\{e\}$-structure an initial G-structure associated to $(\mathcal{E})$. Due to the incredible sizelength-complexity of the underlying computations, this approach almost never abutes: it is forced to incompleteness. But in fact, the main question is to classify.

Classification problem 3.11. Classify systems $(\mathcal{E})$, namely provide complete lists of all possible such equations written in simplified "normal", easily recognizable forms.

Both problems are deeply linked to the classification of Lie algebras of local vector fields. For $n=1, m=1$ and $\kappa=1$, namely $\left(\mathcal{E}_{1}\right): y_{x x}=F\left(x, y, y_{x}\right)$, Lie and Tresse solved the two problems ${ }^{3}$. Table 7 of [O11986], below reproduced, describes the results.

\footnotetext{
${ }^{3}$ The author knows no complete confirmation of the Lie-Tresse classification by means of É. Cartan's method of equivalence.
} 


\begin{tabular}{||l||l|l|l||}
\hline \hline & Symmetry group & Dimension & Invariant equation \\
\hline \hline $\mathbf{( 1 )}$ & & 0 & $y_{x x}=F\left(x, y, y_{x}\right)$ \\
\hline $\mathbf{( 2 )}$ & $\partial_{y}$ & 1 & $y_{x x}=F\left(x, y_{x}\right)$ \\
\hline $\mathbf{( 3 )}$ & $\partial_{x}, \partial_{y}$ & 2 & $y_{x x}=F\left(y_{x}\right)$ \\
\hline $\mathbf{( 4 )}$ & $\partial_{x}, e^{x} \partial_{y}$ & 2 & $y_{x x}-y_{x}=F\left(y_{x}-y\right)$ \\
\hline $\mathbf{( 5 )}$ & $\partial_{x}, \partial_{x}-y \partial_{y}, x^{2} \partial_{x}-2 x y \partial_{y}$ & 3 & $y_{x x}=\frac{3 y_{x}^{2}}{2 y}+c y^{3}$ \\
\hline $\mathbf{( 6 )}$ & $\partial_{x}, x \partial_{x}-y \partial_{y}$, & 3 & $y_{x x}=6 y y_{x}-4 y^{3}+$ \\
& $x^{2} \partial_{x}-(2 x y+1) \partial_{y}$ & & $\left.y_{x}-y^{2}\right)^{3 / 2}$ \\
\hline $\mathbf{( 7 )}$ & $\partial_{x}, \partial_{y}, x \partial_{x}+\alpha y \partial_{y}$, & 3 & $y_{x x}=c\left(y_{x}\right)^{\frac{\alpha-2}{\alpha-1}}$ \\
& $\alpha \neq 0, \frac{1}{2}, 1,2$ & $y_{x x}=c e^{-y_{x}}$ \\
\hline $\mathbf{( 8 )}$ & $\partial_{x}, \partial_{y}, x \partial_{x}+(x+y) \partial_{y}$ & 3 & $y_{x x}=0$ \\
\hline $\mathbf{( 9 )}$ & $\begin{array}{l}\partial_{x}, \partial_{y}, y \partial_{x}, x \partial_{y}, y \partial_{y}, \\
x^{2} \partial_{x}+x y \partial_{y}, x y \partial_{x}+y^{2} \partial_{y}\end{array}$ & 8 & \\
\hline \hline
\end{tabular}

Table 1.

However, the author knows no modern reference offering a complete proof of this classification, with precise insight on the assumptions (some normal forms hold true only at a generic point). In addition, the above Lie-Tresse list is still slightly incomplete in the sense that it does not precise which are the conditions satisfied by $F$ (Table 7 in [O11986]) insuring in the first four lines that $\mathfrak{S Y Y M}\left(\mathcal{E}_{1}\right)$ is indeed of small dimension 0,1 or 2.

Open question 3.12. Specify some precise nondegeneracy conditions upon $F$ in the first four lines of Table 1.

\section{$\S 4$. PunCTUAL AND INFINITESIMAL LIE SYMMETRIES}

4.1. Lie symmetries of $(\mathcal{E})$. Let $\varphi=(\phi, \psi)$ be a diffeomorphism of $\mathbb{K}_{x}^{n} \times \mathbb{K}_{y}^{n}$ as in (1.7)(II).

Definition 4.2. ([Ol1986, Ol1995, BK1989]) $\varphi$ is a (local) Lie symmetry of $(\mathcal{E})$ if it transforms the graph of every solution of $(\mathcal{E})$ into the graph of another solution.

To explain, we must pass to jet spaces. Denote the components of the $(\kappa+1)$-th prolongation $\varphi^{(\kappa+1)}: \mathcal{J}_{n, m}^{\kappa+1} \rightarrow \mathcal{J}_{n, m}^{\kappa+1}$ by

$$
\varphi^{(\kappa+1)}=\left(\phi^{i_{1}}, \psi^{j_{1}}, \Phi_{i_{1}}^{j}, \Phi_{i_{1}, i_{2}}^{j}, \ldots ., \Phi_{i_{1}, i_{2}, \ldots, i_{\kappa+1}}^{j}\right) .
$$

The restriction $\left.\varphi^{(\kappa+1)}\right|_{\Delta_{\mathcal{E}}}$ is obtained by replacing each jet variable $y_{\alpha}^{j}$ by $F_{\alpha}^{j}$, whenever $(j, \alpha) \neq(j, 0)$ and $\neq(j(q), \beta(q))$, and wherever it appears ${ }^{4}$ in the $\Phi_{i_{1}, \ldots, i_{\lambda}}^{j}$.

Let $\pi_{\kappa, p}$ denote the projection from $\mathcal{J}_{n, m}^{\kappa+1}$ to $\Delta_{\mathcal{E}} \simeq \mathbb{K}^{m+n+p}$ defined by

$$
\pi_{\kappa, p}\left(x^{i}, y^{j}, y_{i_{1}}^{j}, \ldots, y_{i_{1}, \ldots, i_{\kappa+1}}^{j}\right):=\left(x^{i}, y^{j}, y_{\beta(q)}^{j(q)}\right)
$$

and introduce the map

$$
\varphi_{\Delta_{\mathcal{E}}}:=\pi_{\kappa, p} \circ\left(\left.\varphi^{(\kappa+1)}\right|_{\Delta_{\mathcal{E}}}\right) \equiv\left(\varphi\left(x^{i}, y^{j}\right), \Phi_{\beta(q)}^{j(q)}\left(x^{i}, y^{j}, y_{\beta\left(q_{1}\right)}^{j\left(q_{1}\right)}\right)\right) .
$$

Lemma 4.6. ([O11986, O11995, BK1989], [*]) The following three conditions are equivalent:

\footnotetext{
${ }^{4}$ Remind from Section 1(II) that we have not (open problem) provided a complete explicit expression of $\Phi_{i_{1}, \ldots, i_{\lambda}}^{j}$ for general $n \geqslant 1, m \geqslant 1$ and $\lambda \geqslant 1$.
} 
(1) the diffeomorphism $\varphi$ is a Lie symmetry of $(\mathcal{E})$;

(2) $\left.\varphi^{(\kappa+1)}\right|_{\Delta_{\mathcal{E}}}$ sends $\Delta_{\mathcal{E}}$ to $\Delta_{\mathcal{E}}$;

(3) $\left.\varphi^{(\kappa+1)}\right|_{\Delta_{\mathcal{E}}}$ sends $\Delta_{\mathcal{E}}$ to $\Delta_{\mathcal{E}}$ and $\varphi_{\Delta_{\mathcal{E}}}=\pi_{\kappa, p}\left(\left.\varphi^{(\kappa+1)}\right|_{\Delta_{\mathcal{E}}}\right)$ is a symmetry of the foliation $\mathrm{F}_{\Delta_{\mathcal{E}}}$, namely it sends every leaf to some other leaf.

Then the set of Lie symmetries of $(\mathcal{E})$ constitutes a local Lie (pseudo)group.

4.7. Infinitesimal Lie symmetries of $(\mathcal{E})$. Let

$$
\mathcal{L}=\sum_{i=1}^{n} \mathcal{X}^{i}(x, y) \frac{\partial}{\partial x^{i}}+\sum_{j=1}^{m} \mathcal{Y}^{j}(x, y) \frac{\partial}{\partial y^{j}},
$$

be a (local) vector field on $\mathbb{K}^{n+m}$ having analytic coefficients. Denote its flow by $\varphi_{t}(x, y):=\exp (t \mathcal{L})(x, y), t \in \mathbb{K}$. As in Section 1(II), by differentiating the prolongation $\left(\varphi_{t}\right)^{(\kappa+1)}$ with respect to $t$ at $t=0$, we get the prolonged vector field $\mathcal{L}^{(\kappa+1)}$ on $\mathcal{J}_{n, m}^{\kappa+1}$, having the general form (Part II):

$$
\mathcal{L}^{(\kappa+1)}=\mathcal{L}+\sum_{j=1}^{m} \sum_{i_{1}=1}^{n} \mathbf{Y}_{i_{1}}^{j} \frac{\partial}{\partial y_{i_{1}}^{j}}+\cdots+\sum_{j=1}^{m} \sum_{i_{1}, \ldots, i_{\kappa+1}=1}^{n} \mathbf{Y}_{i_{1}, \ldots, i_{\kappa+1}}^{j} \frac{\partial}{\partial y_{i_{1}, \ldots, i_{\kappa+1}}^{j}},
$$

with known explicit expressions for the $\mathbf{Y}_{i_{1}, \ldots, i_{\lambda}}^{j}$.

Definition 4.10. $\mathcal{L}$ is an infinitesimal symmetry of $(\mathcal{E})$ if for every small $t$, its time- $t$ flow $\operatorname{map} \varphi_{t}$ is a Lie symmetry of $(\mathcal{E})$.

The restriction $\left.\mathcal{L}^{(\kappa+1)}\right|_{\Delta_{\mathcal{E}}}$ is obtained by replacing every $y_{\alpha}^{j}$ by $F_{\alpha}^{j}$ in all coefficients $\mathbf{Y}_{i_{1}}^{j}, \ldots, \mathbf{Y}_{i_{1}, \ldots, i_{\kappa+1}}^{j}$. Then the coefficients become functions of $\left(x^{i_{1}}, y^{j_{1}}, y_{\beta\left(q_{1}\right)}^{j\left(q_{1}\right)}\right)$ only.

Lemma 4.11. ([O11986, O11995, BK1989], [*]) The following three conditions are equivalent:

(1) the vector field $\mathcal{L}$ is an infinitesimal Lie symmetry of $(\mathcal{E})$;

(2) its $(\kappa+1)$-th prolongation $\mathcal{L}^{(\kappa+1)}$ is tangent to the skeleton $\Delta_{\mathcal{E}}$;

(3) $\mathcal{L}^{(\kappa+1)}$ is tangent to $\Delta_{\mathcal{E}}$ and the push-forward

$$
\mathcal{L}_{\Delta_{\mathcal{E}}}:=\left(\pi_{\kappa, p}\right)_{*}\left(\left.\mathcal{L}^{(\kappa+1)}\right|_{\Delta_{\mathcal{E}}}\right)
$$

is an infinitesimal symmetry of the foliation $\mathrm{F}_{\Delta_{\mathcal{E}}}$, namely for every $i=1, \ldots, n$, the Lie bracket $\left[\mathcal{L}_{\Delta_{\mathcal{E}}}, \mathrm{D}_{i}\right]$ is a linear combination of $\left\{\mathrm{D}_{1}, \ldots, \mathrm{D}_{n}\right\}$.

According to [O11986, BK1989, O11995], the set of infinitesimal Lie symmetries constitutes a Lie algebra, with the property $\left[\mathcal{L}^{(\kappa+1)}, \mathcal{L}^{(\kappa+1)}\right]=\left[\mathcal{L}, \mathcal{L}^{\prime}\right]^{(\kappa+1)}$. We summarize by a diagram.
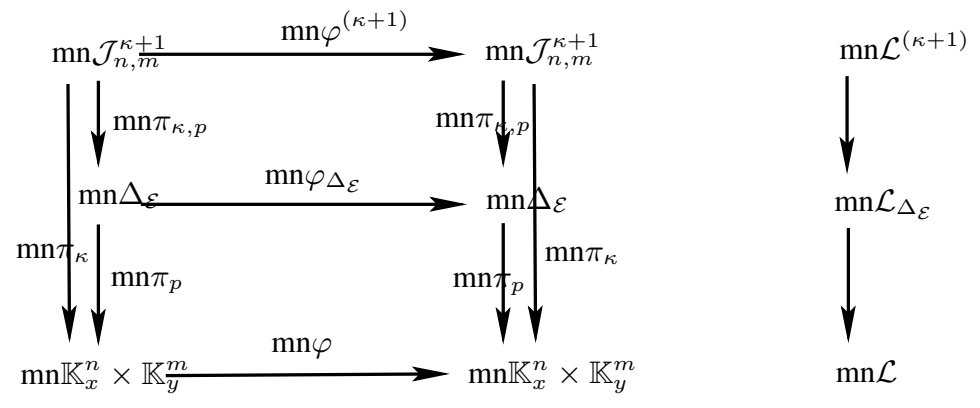
4.13. Sophus Lie's algorithm. We describe the general process. Its complexity will be exemplified in Section 5 (to be read simultaneously).

The tangency of $\mathcal{L}^{(\kappa+1)}$ to $\Delta_{\mathcal{E}}$ is expressed by applying $\mathcal{L}^{(\kappa+1)}$ to the equations $0=$ $-y_{\alpha}^{j}+F_{\alpha}^{j}$, which yields:

$$
0=-\mathbf{Y}_{\alpha}^{j}+\sum_{i=1}^{n} \mathcal{X}^{i} \frac{\partial F_{\alpha}^{j}}{\partial x^{i}}+\sum_{l=1}^{n} \mathcal{Y}^{l} \frac{\partial F_{\alpha}^{j}}{\partial y^{l}}+\sum_{q=1}^{p} \mathbf{Y}_{\beta(q)}^{j(q)} \frac{\partial F_{\alpha}^{j}}{\partial y_{\beta(q)}^{j(q)}},
$$

for $(j, \alpha) \neq(j, 0)$ and $\neq(j(q), \beta(q))$. Restricting a coefficient $\mathbf{Y}_{i_{1}, \ldots, i_{\lambda}}^{j}$ to $\Delta_{\mathcal{E}}$, namely replacing everywhere in it each $y_{\alpha}^{j}$ by $F_{\alpha}^{j}$, provides a specialized coefficient

$$
\widehat{\mathbf{Y}}_{i_{1}, \ldots, i_{\lambda}}^{j}=\widehat{\mathbf{Y}}_{i_{1}, \ldots, i_{\lambda}}^{j}\left(x^{i_{1}}, y^{j_{1}}, y_{\beta\left(q_{1}\right)}^{j\left(q_{1}\right)}, J_{x, y}^{\lambda} \mathcal{X}^{i_{1}}, J_{x, y}^{\lambda} \mathcal{Y}^{j_{1}}\right),
$$

that depends linearly on the $\lambda$-th jet of the coefficients of $\mathcal{L}$, as confirmed by an inspection of Part II's formulas. Here, we use the jet notation $J_{x, y}^{\lambda} \mathcal{Z}:=\left(\partial_{x}^{\alpha_{1}} \partial_{y}^{\beta_{1}} \mathcal{Z}\right)_{\left|\alpha_{1}\right|+\left|\beta_{1}\right| \leqslant \lambda}$. We thus get equations

$$
0 \equiv-\widehat{\mathbf{Y}}_{\alpha}^{j}+\sum_{i=1}^{n} \mathcal{X}^{i} \frac{\partial F_{\alpha}^{j}}{\partial x^{i}}+\sum_{l=1}^{n} \mathcal{Y}^{l} \frac{\partial F_{\alpha}^{j}}{\partial y^{l}}+\sum_{q=1}^{p} \widehat{\mathbf{Y}}_{\beta(q)}^{j(q)} \frac{\partial F_{\alpha}^{j}}{\partial y_{\beta(q)}^{j(q)}},
$$

involving only the variables $\left(x^{i_{1}}, y^{j_{1}}, y_{\beta\left(q_{1}\right)}^{j\left(q_{1}\right)}\right)$.

Next, we develope every such equation with respect to the powers of $y_{\beta\left(q_{1}\right)}^{j\left(q_{1}\right)}$ :

$$
0 \equiv \sum_{\mu_{1}, \ldots, \mu_{p} \geqslant 0}\left(y_{\beta(1)}^{j(1)}\right)^{\mu_{1}} \cdots\left(y_{\beta(p)}^{j(p)}\right)^{\mu_{p}} \Psi_{\alpha, \mu_{1}, \ldots, \mu_{p}}^{j}\left(x^{i_{1}}, y^{j_{1}}, J_{x, y}^{\kappa+1} \mathcal{X}^{i_{1}}, J_{x, y}^{\kappa+1} \mathcal{Y}^{j_{1}}\right) .
$$

The $\Psi_{\alpha, \mu_{1}, \ldots, \mu_{p}}^{j}$ are linear with respect to $\left(J_{x, y}^{\kappa+1} \mathcal{X}^{i_{1}}, J_{x, y}^{\kappa+1} \mathcal{Y}^{j_{1}}\right)$, with certain coefficients analytic with respect to $(x, y)$, which depend intrinsically (but in a complex manner) on the right hand sides $F_{\alpha}^{j}$.

Proposition 4.18. The vector field $\mathcal{L}$ is an infinitesimal Lie symmetry of $(\mathcal{E})$ if and only if its coefficients $\mathcal{X}^{i_{1}}, \mathcal{Y}^{j_{1}}$ satisfy the linear PDE system:

$$
0=\Psi_{\alpha, \mu_{1}, \ldots, \mu_{p}}^{j}\left(x^{i_{1}}, y^{j_{1}}, J_{x, y}^{\kappa+1} \mathcal{X}^{i_{1}}, J_{x, y}^{\kappa+1} \mathcal{Y}^{j_{1}}\right)
$$

for all $(j, \alpha) \neq(j, 0)$ and $\neq(j(q), \beta(q))$ and for all $\left(\mu_{1}, \ldots, \mu_{p}\right) \in \mathbb{N}^{p}$.

In all known instances, a finite number of these equations suffices.

Example 4.20. With $n=m=\kappa=1$, a second prolongation $\mathcal{L}^{(2)}=\mathcal{X} \frac{\partial}{\partial x}+\mathcal{Y} \frac{\partial}{\partial y}+$ $\mathbf{Y}_{1} \frac{\partial}{\partial y_{1}}+\mathbf{Y}_{2} \frac{\partial}{\partial y_{2}}$ is tangent to the skeleton $0=-y_{2}+F\left(x, y, y_{1}\right)$ of $\left(\mathcal{E}_{1}\right)$ if and only if $0=-\mathbf{Y}_{2}+\mathcal{X} F_{x}+\mathcal{Y} F_{y}+\mathbf{Y}_{1} F_{y_{1}}$, or, developing:

$$
\left\{\begin{aligned}
0= & -\mathcal{Y}_{x x}+\left[-2 \mathcal{Y}_{x y}+\mathcal{X}_{x x}\right] y_{1}+\left[-\mathcal{Y}_{y y}+2 \mathcal{X}_{x y}\right]\left(y_{1}\right)^{2}+\left[\mathcal{X}_{y y}\right]\left(y_{1}\right)^{3}+ \\
& +\left[-\mathcal{Y}_{y}+2 \mathcal{X}_{x}\right] F+\left[3 \mathcal{X}_{y}\right] y_{1} F+[\mathcal{X}] F_{x}+[\mathcal{Y}] F_{y}+ \\
& +\left[\mathcal{Y}_{x}\right] F_{y_{1}}+\left[\mathcal{Y}_{y}-\mathcal{X}_{x}\right] y_{1} F_{y_{1}}+\left[-\mathcal{X}_{y}\right]\left(y_{1}\right)^{2} F_{y_{1}}
\end{aligned}\right.
$$

Developing $F=\sum_{k \geqslant 0}\left(y_{1}\right)^{k} F_{k}(x, y)$, we may obtain equations (4.19).

\section{$\S 5$. EXAMPLES}

5.1. Second order ordinary differential equation. Pursuing the study of $\left(\mathcal{E}_{1}\right)$, according to Section 7 below, we may assume that $F=\mathrm{O}\left(y_{x}\right)$, or equivalently $F(x, y, 0) \equiv 0$. 
Convention 5.2. The letters $\mathrm{R}$ will denote various functions of $\left(x, y, y_{1}\right)$, changing with the context. Similarly, $r=r(x, y)$, excluding the pure jet variable $y_{1}$. Hence, symbolically:

$$
\mathbf{R}=\mathbf{r}+y_{1} \mathbf{r}+\left(y_{1}\right)^{2} \mathbf{r}+\left(y_{1}\right)^{3} \mathbf{r}+\cdots .
$$

So the skeleton is

$$
y_{2}=F\left(x, y, y_{1}\right)=y_{1} \mathrm{R}=y_{1} \mathrm{r}+\left(y_{1}\right)^{2} \mathrm{r}+\left(y_{1}\right)^{3} \mathrm{r}+\cdots .
$$

Applying $\mathcal{L}^{(2)}$, see (2.3)(II) for its expression, we get:

$$
0=-\mathbf{Y}_{2}+\mathcal{X} F_{x}+\mathcal{Y} F_{y}+\mathrm{Y}_{1} F_{y_{1}} .
$$

Observe that $F_{x}=\left(y_{1} \mathrm{R}\right)_{x}=\mathrm{r} y_{1}+\mathrm{r}\left(y_{1}\right)^{2}+\cdots$ and similarly for $F_{y}$, but that $\left(y_{1} \mathrm{R}\right)_{y_{1}}=$ $\mathrm{r}+\mathrm{r} y_{1}+\mathrm{r}\left(y_{1}\right)^{2}+\cdots$. Inserting above $\mathrm{Y}_{1}, \mathrm{Y}_{2}$ given by (2.6)(II), replacing $y_{2}$ by $y_{1} \mathrm{R}$ and computing $\bmod \left(y_{1}\right)^{4}$, we get:

$$
\begin{aligned}
0 \equiv & -\mathcal{Y}_{x x}+\left[-2 \mathcal{Y}_{x y}+\mathcal{X}_{x x}\right] y_{1}+\left[-\mathcal{Y}_{y y}+2 \mathcal{X}_{x y}\right]\left(y_{1}\right)^{2}+\left[\mathcal{X}_{y y}\right]\left(y_{1}\right)^{3}+ \\
& +\left[-\mathcal{Y}_{y}+2 \mathcal{X}_{x}\right]\left(y_{1} \mathbf{r}+\left(y_{1}\right)^{2} \mathbf{r}+\left(y_{1}\right)^{3} \mathbf{r}\right)+\left[3 \mathcal{X}_{y}\right]\left(\left(y_{1}\right)^{2} \mathbf{r}+\left(y_{1}\right)^{3} \mathbf{r}\right)+ \\
& +[\mathcal{X}]\left(y_{1} \mathbf{r}+\left(y_{1}\right)^{2} \mathbf{r}+\left(y_{1}\right)^{3} \mathbf{r}\right)+[\mathcal{Y}]\left(y_{1} \mathbf{r}+\left(y_{1}\right)^{2} \mathbf{r}+\left(y_{1}\right)^{3} \mathbf{r}\right)+ \\
& +\left[\mathcal{Y}_{x}\right]\left(\mathbf{r}+y_{1} \mathbf{r}+\left(y_{1}\right)^{2} \mathbf{r}+\left(y_{1}\right)^{3} \mathbf{r}\right)+ \\
& +\left[\mathcal{Y}_{y}-\mathcal{X}_{x}\right]\left(y_{1} \mathbf{r}+\left(y_{1}\right)^{2} \mathbf{r}+\left(y_{1}\right)^{3} \mathbf{r}\right)+\left[-\mathcal{X}_{y}\right]\left(\left(y_{1}\right)^{2} \mathbf{r}+\left(y_{1}\right)^{3} \mathbf{r}\right) .
\end{aligned}
$$

We gather the powers cst., $y_{1},\left(y_{1}\right)^{2}$ and $\left(y_{1}\right)^{3}$, equating their coefficients to 0 :

$$
\begin{aligned}
& 0=-\mathcal{Y}_{x x}+\mathrm{P}\left(\mathcal{Y}_{x}\right), \\
& 0=-2 \mathcal{Y}_{x y}+\mathcal{X}_{x x}+\mathrm{P}\left(\mathcal{Y}_{y}, \mathcal{X}_{x}, \mathcal{X}, \mathcal{Y}, \mathcal{Y}_{x}\right), \\
& 0=-\mathcal{Y}_{y y}+2 \mathcal{X}_{x y}+\mathrm{P}\left(\mathcal{Y}_{y}, \mathcal{X}_{x}, \mathcal{X}_{y}, \mathcal{X}, \mathcal{Y}, \mathcal{Y}_{x}\right), \\
& 0=\mathcal{X}_{y y}+\mathrm{P}\left(\mathcal{Y}_{y}, \mathcal{X}_{x}, \mathcal{X}_{y}, \mathcal{X}, \mathcal{Y}, \mathcal{Y}_{x}\right)
\end{aligned}
$$

Convention 5.8. The letter $P$ will denote various linear combinations of some precise partial derivatives of $\mathcal{X}, \mathcal{Y}$ which have analytic coefficients in $(x, y)$.

By cross-differentiations and substitutions in the above system, all third, fourth, fifth, etc. order derivatives of $\mathcal{X}, \mathcal{Y}$ may be expressed as $\mathrm{P}\left(\mathcal{X}, \mathcal{Y}, \mathcal{X}_{x}, \mathcal{X}_{y}, \mathcal{Y}_{x}, \mathcal{Y}_{y}, \mathcal{Y}_{x y}, \mathcal{Y}_{y y}\right)$.

Proposition 5.9. An infinitesimal Lie symmetry $\mathcal{X} \frac{\partial}{\partial x}+\mathcal{Y} \frac{\partial}{\partial y}$ of $\left(\mathcal{E}_{1}\right)$ is uniquely determined by the eight initial Taylor coefficients:

$$
\mathcal{X}(0), \mathcal{Y}(0), \mathcal{X}_{x}(0), \mathcal{X}_{y}(0), \mathcal{Y}_{x}(0), \mathcal{Y}_{y}(0), \mathcal{Y}_{x y}(0), \mathcal{Y}_{y y}(0)
$$

The bound $\operatorname{dim} \mathfrak{S Y M}\left(\mathcal{E}_{1}\right) \leqslant 8$ is attained with $F=0$, whence all $\mathrm{P}=0$ and

$$
\begin{cases}A:=\partial_{y}, & E:=y \partial_{y}, \\ B:=\partial_{x}, & F:=y \partial_{x}, \\ C:=x \partial_{y}, & G:=x x \partial_{x}+x y \partial_{y}, \\ D:=x \partial_{x}, & H:=x y \partial_{x}+y y \partial_{y} .\end{cases}
$$

are infinitesimal generators of the group $\operatorname{PGL}_{3}(\mathbb{K})=\operatorname{Aut}\left(P_{2}(\mathbb{K})\right)$ of projective transformations

$$
(x, y) \mapsto\left(\frac{\alpha x+\beta y+\gamma}{\lambda x+\mu y+\nu}, \frac{\delta x+\eta y+\epsilon}{\lambda x+\mu y+\nu},\right)
$$

stabilizing the collections of all affine lines of $\mathbb{K}^{2}$, namely the solutions of the model equation $y_{x x}=0$. The model Lie algebra $\mathfrak{p g l}_{3}(\mathbb{K}) \simeq \mathfrak{s l}_{3}(\mathbb{K})$ is simple. 
Theorem 5.13. The bound $\operatorname{dim} \mathfrak{S P M}\left(\mathcal{E}_{1}\right) \leqslant 8$ is attained if and only if $\left(\mathcal{E}_{1}\right)$ is equivalent, through a diffeomorphism $(x, y) \mapsto(X, Y)$, to $Y_{X X}=0$.

Proof. The statement is well known ([Lie1883, EL1890, Tr1896, Se1931, Ca1932a, O11986, HK1989, Ib1992, O11995, Sh1997, Su2001, N2003, Me2004]). We provide a (new?) proof which has the advantage to enjoy direct generalizations to all PDE systems whose model Lie algebras are semisimple, for instance $\left(\mathcal{E}_{2}\right),\left(\mathcal{E}_{3}\right)$ and $\left(\mathcal{E}_{5}\right)$.

The Lie brackets between the eight generators (5.11) are:

\begin{tabular}{l||l|l|l|l|l|l|l|l} 
& $A$ & $B$ & $C$ & $D$ & $E$ & $F$ & $G$ & $H$ \\
\hline \hline$A$ & 0 & 0 & 0 & 0 & $A$ & $B$ & $C$ & $D+2 E$ \\
\hline$B$ & 0 & 0 & $A$ & $B$ & 0 & 0 & $E+2 D$ & $F$ \\
\hline$C$ & 0 & $-A$ & 0 & $-C$ & $C$ & $D-E$ & 0 & $G$ \\
\hline$D$ & 0 & $-B$ & $C$ & 0 & 0 & $-F$ & $G$ & 0 \\
\hline$E$ & $-A$ & 0 & $-C$ & 0 & 0 & $F$ & 0 & $H$ \\
\hline$F$ & $-B$ & 0 & $-D+E$ & $F$ & $-F$ & 0 & $H$ & 0 \\
\hline$G$ & $-C$ & $-E-2 D$ & 0 & $-G$ & 0 & $H$ & 0 & 0 \\
\hline$H$ & $-D-2 E$ & $-F$ & $-G$ & 0 & $-H$ & 0 & 0 & 0
\end{tabular}

Table 2.

Assuming that $\operatorname{dim} \mathfrak{S Y M M}\left(\mathcal{E}_{1}\right)=8$, taking account of (5.7), after making some linear combinations, there must exist eight generators of the form

$$
\begin{cases}A^{\prime}:=\partial_{y}+\mathrm{O}(1), & E^{\prime}:=y \partial_{y}+\mathrm{O}(2), \\ B^{\prime}:=\partial_{x}+\mathrm{O}(1), & F^{\prime}:=y \partial_{x}+\mathrm{O}(2), \\ C^{\prime}:=x \partial_{y}+\mathrm{O}(2), & G^{\prime}:=x x \partial_{x}+x y \partial_{y}+\mathrm{O}(3), \\ D^{\prime}:=x \partial_{x}+\mathrm{O}(2), & H^{\prime}:=x y \partial_{x}+y y \partial_{y}+\mathrm{O}(3) .\end{cases}
$$

To insure that the Lie brackets between these vector fields are small perturbations of the model ones, we can in advance replace $(x, y)$ by $(\varepsilon x, \varepsilon y)$, so that $y_{x x}=\varepsilon F\left(\varepsilon x, \varepsilon y, y_{x}\right)$ is an $\mathrm{O}(\varepsilon)$, hence all the remainders $\mathrm{O}(1), \mathrm{O}(2)$ and $\mathrm{O}(3)$ above are also $\mathrm{O}(\varepsilon)$. It follows that the structure constants for $A^{\prime}, \ldots, H^{\prime}$ are $\varepsilon$-close to those of Table 2.

Theorem 5.15. ([OV1994]) Every semisimple Lie algebra over $\mathbb{R}$ or $\mathbb{C}$ is rigid: small deformations of the structure constants just give isomorphic Lie algebras.

Consequently, there exists a change of basis close to the identity leading to new generators $A^{\prime \prime}, B^{\prime \prime}, \ldots, G^{\prime \prime}, H^{\prime \prime}$ having exactly the same structure constants as in Table 2 . Then $A^{\prime \prime}(0)$ and $B^{\prime \prime}(0)$ are still linearly independent. Since $\left[A^{\prime \prime}, B^{\prime \prime}\right]=[A, B]=0$, there exist local coordinates $(X, Y)$ centered at 0 in which $A^{\prime \prime}=\partial_{X}$ and $B^{\prime \prime}=\partial_{Y}$. Since $\left[A^{\prime \prime}, C^{\prime \prime}\right]=[A, C]=0$ and $\left[B^{\prime \prime}, C^{\prime \prime}\right]=[B, C]=A$, it follows that $C^{\prime \prime}=X \partial_{Y}$. The tangency to $0=-Y_{2}+F\left(X, Y, Y_{1}\right)$ (with $F(0)=0$ ) of $\left(\partial_{X}\right)^{(2)}=\partial_{X}$, of $\left(\partial_{Y}\right)^{(2)}=\partial_{Y}$ and of $\left(X \partial_{Y}\right)^{(2)}=X \partial_{Y}+\partial_{Y_{1}}$ yields $F=0$.

Open question 5.16. Does this proof generalize to $y_{x^{\kappa+1}}=F\left(x, y, y_{x}, \ldots, y_{x^{\kappa}}\right)$ ?

5.17. Complete system of second order. We now summarize a generalization to $\left(\mathcal{E}_{2}\right)$. According to Section 7 below, one may assume that the submanifold of solutions is $y=b+\sum_{i=1}^{n} a^{i}\left[x^{i}+\mathrm{O}\left(|x|^{2}\right)+\mathrm{O}(a)+\mathrm{O}(b)\right]$, whence $y_{x^{i_{1}} x^{i_{2}}}=F_{i_{1}, i_{2}}\left(x^{i}, y, y_{x^{k}}\right)$ with $F(x, y, 0) \equiv 0$. Applying to the skeleton $0=-y_{i_{1}, i_{2}}+F_{i_{1}, i_{2}}\left(x^{i}, y, y_{k}\right)$ a second 
prolongation $\mathcal{L}^{(2)}$ having coefficients $\mathbf{Y}_{i_{1}}$ given by (3.9)(II) and $\mathbf{Y}_{i_{1}, i_{2}}$ given by (3.20)(II), we get

$$
0=-\mathbf{Y}_{i_{1}, i_{2}}+\sum_{k=1}^{n}\left[\mathcal{X}^{k}\right] \frac{\partial F_{i_{1}, i_{2}}}{\partial x^{k}}+[\mathcal{Y}] \frac{\partial F_{i_{1}, i_{2}}}{\partial y}+\sum_{k=1}^{n}\left[\mathbf{Y}_{k}\right] \frac{\partial F_{i_{1}, i_{2}}}{\partial y_{k}}
$$

Replacing $y_{i_{1}, i_{2}}$ everywhere by $F_{i_{1}, i_{2}}=y_{1} \mathrm{R}+\cdots+y_{n} \mathrm{R}$, developping in powers of the pure jet variables $y_{l}$ and picking the coefficients of cst., of $y_{k}$, of $\left(y_{k}\right)^{2}$ and of $\left(y_{k}\right)^{3}$, we get the linear system

$$
\left\{\begin{aligned}
\mathcal{Y}_{x^{i_{1}} x^{i_{2}}} & =\mathrm{P}\left(\mathcal{Y}_{x^{l}}\right) \\
\delta_{i_{1}}^{k} \mathcal{Y}_{x^{i_{2}}}+\delta_{i_{2}}^{k} \mathcal{Y}_{x^{i_{1} y}}-\mathcal{X}_{x^{i_{1}} x^{i_{2}}}^{k} & =\mathrm{P}\left(\mathcal{Y}_{y}, \mathcal{X}_{x^{l_{1}}}^{l_{2}}, \mathcal{X}^{l}, \mathcal{Y}, \mathcal{Y}_{x^{l}}\right) \\
\delta_{i_{1}, i_{2}}^{k, k} \mathcal{Y}_{y y}-\delta_{i_{1}}^{k} \mathcal{X}_{x^{i_{2}} y}^{k}-\delta_{i_{2}}^{k} \mathcal{X}_{x^{i_{1} y}}^{k} & =\mathrm{P}\left(\mathcal{Y}_{y}, \mathcal{X}_{x^{l_{1}}}^{l_{2}}, \mathcal{X}_{y}^{l}, \mathcal{X}^{l}, \mathcal{Y}, \mathcal{Y}_{x^{l}}\right) \\
\delta_{i_{1}, i_{2}}^{k, k} \mathcal{X}_{y y}^{k} & =\mathrm{P}\left(\mathcal{Y}_{y}, \mathcal{X}_{x^{l_{1}}}^{l_{2}}, \mathcal{X}_{y}^{l}, \mathcal{X}^{l}, \mathcal{Y}, \mathcal{Y}_{x^{l}}\right)
\end{aligned}\right.
$$

upon which obvious linear combinations yield a known generalization of Proposition 5.9.

Proposition 5.20. ([Su2001, GM2003a]) An infinitesimal Lie symmetry $\sum_{k=1}^{n} \mathcal{X}^{k} \frac{\partial}{\partial x^{k}}+$ $\mathcal{Y} \frac{\partial}{\partial y}$ is uniquely determined by the $n^{2}+4 n+3$ initial Taylor coefficients:

$$
\mathcal{X}^{l}(0), \mathcal{Y}(0), \mathcal{X}_{x^{l_{1}}}^{l_{2}}(0), \mathcal{X}_{y}^{l}(0), \mathcal{Y}_{x^{l}}(0), \mathcal{Y}_{y}(0), \mathcal{Y}_{x^{l} y}(0), \mathcal{Y}_{y y}(0)
$$

The bound $\operatorname{dim} \mathfrak{S P M}\left(\mathcal{E}_{2}\right) \leqslant n^{2}+4 n+3$ is attained with $F_{i_{1}, i_{2}}=0$, whence all $\mathrm{P}=0$ and

$$
\left\{\begin{aligned}
A & :=\partial_{y}, & E & :=y \partial_{y}, \\
B_{i} & :=\partial_{x^{i}}, & F_{i} & :=y \partial_{x^{i}}, \\
C_{i} & :=x^{i} \partial_{y}, & G_{i} & :=x^{i}\left(x^{1} \partial_{x^{1}}+\cdots+x^{n} \partial_{x^{n}}+y \partial_{y}\right)+x y \partial_{y}, \\
D_{i, k} & :=x^{i} \partial_{x^{k}}, & H & :=y\left(x^{1} \partial_{x^{1}}+\cdots+x^{n} \partial_{x^{n}}+y \partial_{y}\right) .
\end{aligned}\right.
$$

are infinitesimal generators of the group $\operatorname{PGL}_{n+2}(\mathbb{K})=\operatorname{Aut}\left(P_{n+1}(\mathbb{K})\right)$ of projective transformations

$$
(x, y) \mapsto\left(\frac{\alpha_{1} x^{1}+\cdots+\alpha_{n} x^{n}+\beta y+\gamma}{\lambda_{1} x^{1}+\cdots+\lambda_{n} x^{n}+\mu y+\nu}, \frac{\delta_{1} x^{1}+\cdots+\delta_{n} x^{n}+\eta y+\epsilon}{\lambda_{1} x^{1}+\cdots+\lambda_{n} x^{n}+\mu y+\nu},\right)
$$

stabilizing the collections of all affine planes of $\mathbb{K}^{n+1}$, namely the solutions of the model equation $y_{x^{i_{1}} x^{i_{2}}}=0$. The model Lie algebra $\mathfrak{p g l}_{n+2}(\mathbb{K}) \simeq \mathfrak{s l}_{n+2}(\mathbb{K})$ is simple, hence rigid.

Theorem 5.24. The bound $\operatorname{dim} \mathfrak{S P M}\left(\mathcal{E}_{2}\right) \leqslant n^{2}+4 n+3$ is attained if and only if $\left(\mathcal{E}_{2}\right)$ is equivalent, through a diffeomorphism $\left(x^{i}, y\right) \mapsto\left(X^{k}, Y\right)$, to $Y_{X^{k_{1}} X^{k_{2}}}=0$.

The proof, similar to that of Theorem 5.13, is skipped.

The study of $\left(\mathcal{E}_{3}\right)$ also leads to the model algebra $\mathfrak{p g l}_{n+2}(\mathbb{K}) \simeq \mathfrak{s l}_{n+2}(\mathbb{K})$ and an analog to Theorem 5.13 holds. Details are similar.

\section{§6. TRANSFER OF LIE SYMMETRIES TO THE PARAMETER SPACE}

6.1. Stabilization of foliations. As announced in $\S 2.38$, we now transfer the theory of Lie symmetries to submanifolds of solutions.

Restarting from $\S 4.1$, let $\varphi$ a Lie symmetry of $(\mathcal{E})$, namely $\varphi_{\Delta_{\mathcal{E}}}$ stabilizes $\mathrm{F}_{\Delta_{\mathcal{E}}}$. The diffeomorphism $A$ defined by (2.9) transforms $F_{v}$ to $F_{\Delta_{\mathcal{E}}}$. Conjugating, we get the selftransformation $\mathrm{A}^{-1} \circ \varphi_{\Delta_{\mathcal{E}}} \circ \mathrm{A}$ of the $(x, a, b)$-space that must stabilize also the foliation $\mathrm{F}_{\mathrm{v}}$. Equivalently, it must have expression:

$$
\left[\mathrm{A}^{-1} \circ \varphi_{\Delta_{\mathcal{E}}} \circ \mathrm{A}\right](x, a, b)=(\theta(x, a, b), f(a, b), g(a, b)) \in \mathbb{K}^{n} \times \mathbb{K}^{p} \times \mathbb{K}^{m},
$$


where, importantly, the last two components are independent of the coordinate $x$, because the leaves of $F_{v}$ are just $\{a=$ cst., $b=$ cst $\}$.

Lemma 6.3. To every Lie symmetry $\varphi$ of $(\mathcal{E})$, there corresponds a transformation of the parameters

$$
(a, b) \longmapsto(f(a, b), g(a, b))=: h(a, b)
$$

meaning that $\varphi$ transforms the local solution $y_{a, b}(x):=\Pi(x, a, b)$ to the local solution $y_{h(a, b)}(x)=\Pi(x, h(a, b))$.

Unfortunately, the expression of $\mathrm{A}^{-1} \circ \varphi_{\Delta_{\mathcal{E}}} \circ \mathrm{A}$ does not clearly show that $f$ and $g$ are independent of $x$. Indeed, reminding the expressions of $\mathrm{A}$ and of $\Phi$, we have:

$$
\varphi_{\Delta_{\mathcal{E}}} \circ \mathrm{A}(x, a, b)=\left(\varphi(x, \Pi(x, a, b)), \Phi_{\beta(q)}^{j(q)}\left(x^{i_{1}}, \Pi^{j_{1}}(x, a, b), \Pi_{x^{\beta\left(q_{1}\right)}}^{j\left(q_{1}\right)}(x, a, b)\right)\right) .
$$

To compose with $A^{-1}$ whose expression is given by (2.21), it is useful to split $\varphi=$ $(\phi, \psi) \in \mathbb{K}^{n} \times \mathbb{K}^{m}$, so above we write

$$
\varphi(x, \Pi(x, a, b))=(\phi(x, \Pi(x, a, b)), \psi(x, \Pi(x, a, b))),
$$

and finally, droping the arguments:

$$
\left[\mathrm{A}^{-1} \circ \varphi_{\Delta_{\mathcal{E}}} \circ \mathrm{A}\right](x, a, b)=\left(\phi^{i}, A^{q}\left(\phi^{i_{1}}, \psi^{j_{1}}, \Phi_{\beta\left(q_{1}\right)}^{j\left(q_{1}\right)}\right), B^{j}\left(\phi^{i_{1}}, \psi^{j_{1}}, \Phi_{\beta\left(q_{1}\right)}^{j\left(q_{1}\right)}\right)\right) .
$$

In case $(\mathcal{E})=\left(\mathcal{E}_{1}\right)$, is an exercise to verify by computations that the $A^{q}(\cdot)$ and $B^{j}(\cdot)$ are independent of $x$. In general however, the explicit expression of $\Phi_{i_{1}, \ldots, i_{\lambda}}^{j}$ is unknown. Unfortunately also, nothing shows how $(f(a, b), g(a, b))$ is uniquely associated to $\varphi(x, y)$. Further explanations are needed.

6.8. Determination of parameter transformations. At first, we state a geometric reformulation of the preceding lemma.

Lemma 6.9. Every Lie symmetry $(x, y) \mapsto \varphi(x, y)$ of $(\mathcal{E})$ induces a local $\mathbb{K}$-analytic diffeomorphism

$$
(x, y, a, b) \longmapsto(\varphi(x, y), h(a, b))
$$

of $\mathbb{K}_{x}^{n} \times \mathbb{K}_{y}^{m} \times \mathbb{K}_{a}^{p} \times \mathbb{K}_{b}^{m}$ that maps to itself the associated submanifold of solutions

$$
\mathcal{M}_{\mathcal{E}}=\{(x, y, a, b): y=\Pi(x, a, b)\} .
$$

Proof. In fact, we know that the $n$-dimensional leaf $\left\{(x, \Pi(x, a, b)): x \in \mathbb{K}^{n}\right\}$ is sent $\left\{(x, \Pi(x, h(a, b))): x \in \mathbb{K}^{n}\right\}$.

Equivalently, setting $c:=(a, b)$ and writing $(\varphi, h)=(\phi, \psi, h)$, we have $\psi=\Pi(\phi, h)$ when $y=\Pi(x, c)$, namely

$$
\psi(x, \Pi(x, c)) \equiv \Pi(\phi(x, \Pi(x, c)), h(c))
$$

Proposition 6.13. There exists a universal rational map $\widehat{\mathrm{H}}$ such that

$$
h(c) \equiv \widehat{\mathrm{H}}\left(J_{x, a, b}^{\kappa+1} \Pi(x, c), J_{x, y}^{\kappa} \varphi(x, \Pi(x, c))\right)
$$

This shows unique determination of $h$ from $\varphi$, given $(\mathcal{E})$ or equivalently, given $\Pi$. 
Proof. Differentiating a function $\chi(x, \Pi(x, c))$ with respect to $x^{k}, k=1, \ldots, n$, corresponds to applying to $\chi$ the vector field

$$
\mathrm{L}_{k}:=\frac{\partial}{\partial x_{k}}+\sum_{j=1}^{m} \frac{\partial \Pi^{j}}{\partial x_{k}}(x, c) \frac{\partial}{\partial y^{j}}, \quad k=1, \ldots, n .
$$

Thus, applying $\mathrm{L}_{k}$ to the $m$ scalar equations (6.12), we get

$$
\mathrm{L}_{k} \psi^{j}=\sum_{l=1}^{n} \frac{\partial \Pi^{j}}{\partial x^{l}} \mathrm{~L}_{k} \phi^{l}
$$

for $1 \leqslant k \leqslant n$ and $1 \leqslant j \leqslant m$. It follows from the assumption that $\varphi$ is a local diffeomorphism that $\operatorname{det}\left(\mathrm{L}_{k} \phi^{l}(0)\right)_{1 \leqslant k \leqslant n}^{1 \leqslant l \leqslant n} \neq 0$ also. So we may solve the first derivatives $\Pi_{x}$ above: there exist universal polynomials $S_{l}^{j}$ such that

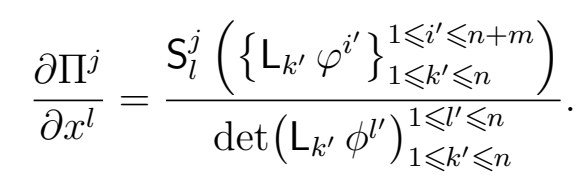

Again, we apply the $L_{k}$ to these equations, getting, thanks to the chain rule:

$$
\sum_{l_{2}=1}^{n} \frac{\partial^{2} \Pi^{j}}{\partial x^{l_{1}} x^{l_{2}}} \mathrm{~L}_{k} \phi^{l_{2}}=\frac{\mathrm{R}_{l_{1}, k}^{j}\left(\left\{\mathrm{~L}_{k_{1}^{\prime}} \mathrm{L}_{k_{2}^{\prime}} \varphi^{i^{\prime}}\right\}_{1 \leqslant k_{1}^{\prime}, k_{2}^{\prime} \leqslant n}^{1 \leqslant i^{\prime} \leqslant n+m}\right)}{\left[\operatorname{det}\left(\mathrm{L}_{k^{\prime}} \phi^{l^{\prime}}\right)_{\substack{1 \leqslant l^{\prime} \leqslant n \\ 1 \leqslant k^{\prime} \leqslant n}}^{2}\right.}
$$

Here, $\mathrm{R}_{l_{1}, k}^{j}$ are universal polynomials. Solving the second derivatives $\Pi_{x^{l_{1}} x^{l_{2}}}^{j}$, we get

$$
\frac{\partial^{2} \Pi^{j}}{\partial x^{l_{1}} x^{l_{2}}}=\frac{\mathrm{S}_{l_{1}, l_{2}}^{j}\left(\left\{\mathrm{~L}_{k_{1}^{\prime}} \mathrm{L}_{k_{2}^{\prime}} \varphi^{i^{\prime}}\right\}_{1 \leqslant k_{1}^{\prime}, k_{2}^{\prime} \leqslant n}^{1 \leqslant i^{\prime} \leqslant n+m}\right)}{\left[\operatorname{det}\left(\mathrm{L}_{k^{\prime}} \phi^{l^{\prime}}\right)_{1 \leqslant k^{\prime} \leqslant n}^{1 \leqslant l^{\prime} \leqslant n}\right]^{3}}
$$

By induction, for every $\beta \in \mathbb{N}^{n}$ :

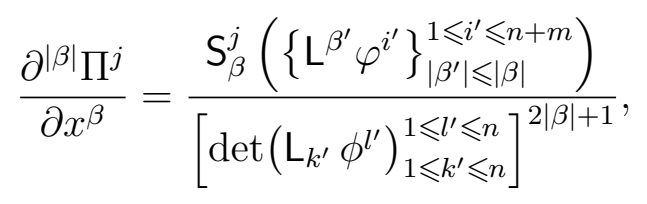

where $S_{\beta}^{j}$ are universal polynomials. Here, for $\beta^{\prime} \in \mathbb{N}^{n}$, we denote by $L^{\beta^{\prime}}$ the derivation of order $\left|\beta^{\prime}\right|$ defined by $\left(L_{1}\right)^{\beta_{1}^{\prime}} \cdots\left(L_{n}\right)^{\beta_{n}^{\prime}}$.

Next, thanks to the assumption that $\mathcal{M}$ is solvable with respect to the parameters, there exist integers $j(1), \ldots, j(p)$ with $1 \leqslant j(q) \leqslant m$ and multiindices $\beta(1), \ldots, \beta(p) \in \mathbb{N}^{n}$ with $|\beta(q)| \geqslant 1$ and $\max _{1 \leqslant q \leqslant p}|\beta(q)|=\kappa$ such that the local $\mathbb{K}$-analytic map

$$
\mathbb{K}^{p+m} \ni c \longmapsto\left(\left(\Pi^{j}(0, c)\right)^{1 \leqslant j \leqslant m},\left(\frac{\partial^{|\beta(q)|} \Pi^{j(q)}}{\partial x^{\beta(q)}}(0, c)\right)_{1 \leqslant q \leqslant p}\right) \in \mathbb{K}^{p+m}
$$

has rank $p+m$ at $c=0$. We then consider in (6.20) only the $(p+m)$ equations written for $(j, 0),(j(q), \beta(q))$ and we solve $h(c)$ by means of the analytic implicit function theorem:

$$
h=\widehat{H}\left(\phi, \frac{\mathrm{S}_{\beta(1)}^{j(1)}\left(\left\{\mathrm{L}^{\beta^{\prime}} \varphi^{i^{\prime}}\right\}_{\left|\beta^{\prime}\right| \leqslant|\beta(1)|}^{1 \leqslant i^{\prime} \leqslant n+m}\right)}{\operatorname{det}\left[\left(\mathrm{L}_{k^{\prime}} \phi^{l^{\prime}}\right)_{1 \leqslant k^{\prime} \leqslant n}^{1 \leqslant l^{\prime} \leqslant n}\right]^{2|\beta(1)|+1}}, \ldots, \frac{\mathrm{S}_{\beta(p)}^{j(p)}\left(\left\{\mathrm{L}^{\beta^{\prime}} \varphi^{i^{\prime}}\right\}_{\left|\beta^{\prime}\right| \leqslant|\beta(p)|}^{1 \leqslant i^{\prime} \leqslant n+m}\right)}{\operatorname{det}\left[\left(\mathrm{L}_{k^{\prime}} \phi^{l^{\prime}}\right)_{1 \leqslant k^{\prime} \leqslant n}^{1 \leqslant l^{\prime} \leqslant n}\right]^{2|\beta(p)|+1}}\right) .
$$


Finally, by developping every derivative $\mathrm{L}^{\beta^{\prime}} \varphi^{i^{\prime}}$ (including $\mathrm{L}_{k^{\prime}} \phi^{l^{\prime}}$ as a special case), taking account of the fact that the coefficients of the $L_{k^{\prime}}$ depend directly on $\Pi$, we get some universal polynomial $\mathrm{P}_{\beta^{\prime}}\left(J_{x}^{\left|\beta^{\prime}\right|+1} \Pi, J_{x, y}^{\left|\beta^{\prime}\right|} \varphi^{i^{\prime}}\right)$. Inserting above, we get the map $\widehat{\mathrm{H}}$.

6.23. Pseudogroup of twin transformations. The previous considerations lead to introducing the following.

Definition 6.24. By $G_{v, p}$, we denote the infinite-dimensional (pseudo)group of local $\mathbb{K}$ analytic diffeomorphisms

$$
(x, y, a, b) \longmapsto(\varphi(x, y), h(a, b))
$$

that respect the separation between the variables and the parameters.

A converse to Lemma 6.3 holds.

Lemma 6.26. Let $\mathcal{M}$ be a submanifold $y=\Pi(x, a, b)$ that is solvable with respect to the parameters $(a, b)$. If a local $\mathbb{K}$-analytic diffeomorphism $(x, y, a, b) \longmapsto(\varphi(x, y), h(a, b))$ of $\mathbb{K}_{x}^{n} \times \mathbb{K}_{y}^{m} \times \mathbb{K}_{a}^{p} \times \mathbb{K}_{b}^{m}$ belonging to $\mathrm{G}_{\mathrm{v}, \mathrm{p}}$ sends $\mathcal{M}$ to $\mathcal{M}$, then $(x, y) \mapsto \varphi(x, y)$ is a Lie symmetry of the PDE system $\mathcal{E}_{\mathcal{M}}$ associated to $\mathcal{M}$.

Proof. In fact, since $(\varphi, h)$ respects the separation of variables and stabilizes $\mathcal{M}$, it respects the fundamental pair of foliations $\left(\mathrm{F}_{\mathrm{v}}, \mathrm{F}_{\mathrm{p}}\right)$, namely $\left\{(a, b)=\left(a_{0}, b_{0}\right)\right\} \cap \mathcal{M}$ is sent to $\left\{(a, b)=h\left(a_{0}, b_{0}\right)\right\} \cap \mathcal{M}$ and $\left\{(x, y)=\left(x_{0}, y_{0}\right)\right\} \cap \mathcal{M}$ is sent to $\{(x, y)=$ $\left.\varphi\left(x_{0}, y_{0}\right)\right\} \cap \mathcal{M}$. Hence $\varphi_{\Delta_{\mathcal{E}_{\mathcal{M}}}}$ also stabilizes $\mathrm{F}_{\Delta_{\mathcal{E}}}$.

Corollary 6.27. Through the one-to-one correspondence $(\mathcal{E}) \longleftrightarrow \mathcal{M}$ of Proposition 2.17, Lie symmetries of $(\mathcal{E})$ correspond to elements of $\mathrm{G}_{\mathrm{v}, \mathrm{p}}$ which stabilize $\mathcal{M}$.

Definition 6.28. Let $\operatorname{Aut}_{\mathrm{v}, \mathrm{p}}(\mathcal{M})$ denote the local (pseudo)group of $(\varphi, h) \in \mathrm{G}_{\mathrm{v}, \mathrm{p}}$ stabilizing $\mathcal{M}$. Let $\operatorname{Lie}(\mathcal{E})$ denote the local (pseudo)group of Lie symmetries of $(\mathcal{E})$.

In summary:

$$
\operatorname{Lie}(\mathcal{E}) \simeq \operatorname{Aut}_{\mathrm{v}, \mathrm{p}}\left(\mathcal{M}_{(\mathcal{E})}\right) \quad \text { and } \quad \operatorname{Aut}_{\mathrm{v}, \mathrm{p}}(\mathcal{M}) \simeq \operatorname{Lie}\left(\mathcal{E}_{\mathcal{M}}\right)
$$

6.30. Transfer of infinitesimal Lie symmetries. Let $\mathcal{L} \in \mathfrak{S Y Y M}(\mathcal{E})$, i.e. $\mathcal{L}_{\Delta_{\mathcal{E}}}$ is tangent to $\Delta_{\mathcal{E}}$. Through the diffeomorphism $\mathrm{A}$, the push-forward of $\mathcal{L}_{\Delta_{\mathcal{E}}}$ must be of the form

$$
\mathrm{A}_{*}^{-1}\left(\mathcal{L}_{\Delta_{\mathcal{E}}}\right)=\sum_{k=1}^{n} \Theta^{i}(x, a, b) \frac{\partial}{\partial x^{i}}+\sum_{q=1}^{p} \mathcal{F}^{q}(a, b) \frac{\partial}{\partial a^{q}}+\sum_{j=1}^{m} \mathcal{G}^{j}(a, b) \frac{\partial}{\partial b^{j}},
$$

where the last two families of $\mathbb{K}$-analytic coefficients $\mathcal{F}^{q}$ and $\mathcal{G}^{j}$ depend only on $(a, b)$.

Lemma 6.32. To every infinitesimal symmetry $\mathcal{L}$ of $(\mathcal{E})$, we can associate an infinitesimal symmetry

$$
\mathcal{L}^{*}:=\sum_{q=1}^{p} \mathcal{F}^{q}(a, b) \frac{\partial}{\partial a^{q}}+\sum_{j=1}^{m} \mathcal{G}^{j}(a, b) \frac{\partial}{\partial b^{j}}
$$

of the space of parameters which tells how the flow of $\mathcal{L}$ acts infinitesimally on the leaves of $\mathrm{F}_{\Delta_{\mathcal{E}}}$. Furthermore, $\mathcal{L}+\mathcal{L}^{*}$ is tangent to the submanifold of solutions $\mathcal{M}_{(\mathcal{E})}$.

Considering the flow of $\mathcal{L}+\mathcal{L}^{*}$ reduces these assertions and the next to the arguments of the preceding paragraphs. So we summarize. 
Lemma 6.34. Let $\mathcal{M}$ be a submanifold $y=\Pi(x, a, b)$ that is solvable with respect to the parameters $(a, b)$. If a vector field that respects the separation between variables and parameters, namely of the form

$$
\mathcal{L}+\mathcal{L}^{*}=\sum_{i=1}^{n} \mathcal{X}^{i}(x, y) \frac{\partial}{\partial x^{i}}+\sum_{j=1}^{m} \mathcal{Y}^{j}(x, y) \frac{\partial}{\partial y^{j}}+\sum_{q=1}^{p} \mathcal{F}^{q}(a, b) \frac{\partial}{\partial a^{q}}+\sum_{j=1}^{m} \mathcal{G}^{j}(a, b) \frac{\partial}{\partial b^{j}}
$$

is tangent to $\mathcal{M}$, then $\mathcal{L}$ is an infinitesimal Lie symmetry of $\left(\mathcal{E}_{\mathcal{M}}\right)$

Corollary 6.36. Through the one-to-one correspondence $(\mathcal{E}) \longleftrightarrow \mathcal{M}$ of Proposition 2.17, infinitesimal Lie symmetries of $(\mathcal{E})$ correspond to vector fields $\mathcal{L}+\mathcal{L}^{*}$ tangent to $\mathcal{M}$.

Definition 6.37. Let $\mathfrak{S Y M M}(\mathcal{M})$ denote the Lie algebra of vector fields $\mathcal{L}+\mathcal{L}^{*}$ tangent to $\mathcal{M}$. Let $\mathfrak{S} \mathfrak{Y} \mathfrak{M}(\mathcal{E})$ denote the Lie algebra of infinitesimal Lie symmetries of $(\mathcal{E})$.

In summary:

$$
\mathfrak{S P M}(\mathcal{E}) \simeq \mathfrak{S Y M}\left(\mathcal{M}_{(\mathcal{E})}\right) \quad \text { and } \quad \operatorname{SYMM}(\mathcal{M}) \simeq \mathfrak{S P M}\left(\mathcal{E}_{\mathcal{M}}\right)
$$

6.39. Dual defining equations. As in $\S 2.10$, let $\mathcal{M} \subset \mathbb{K}_{x}^{n} \times \mathbb{K}_{y}^{m} \times \mathbb{K}_{a}^{p} \times \mathbb{K}_{b}^{m}$ given by $0=-y^{j}+\Pi^{j}(x, a, b)$ and assume if to be solvable with respect to the parameters. In particular, we can solve the $b^{j}$, obtaining dual defining equations

$$
b^{j}=\Pi^{* j}(a, x, y), \quad j=1, \ldots, m,
$$

for some local $\mathbb{K}$-analytic map map $\Pi^{*}=\left(\Pi^{* 1}, \ldots, \Pi^{* m}\right)$ satisfying

$$
b \equiv \Pi^{*}(a, x, \Pi(x, a, b)) \quad \text { and } \quad y \equiv \Pi\left(x, a, \Pi^{*}(a, x, y)\right) .
$$

6.42. An algorithm for the computation of $\mathfrak{S P M}(\mathcal{M})$. The tangency to $\mathcal{M}$ is expressed by applying the vector field (6.35) to $0=-y^{j}+\Pi^{j}(x, a, b)$, which yields:

$$
\begin{aligned}
0=-\mathcal{Y}^{j}(x, y)+\sum_{i=1}^{n} \mathcal{X}^{i}(x, y) \Pi_{x^{i}}^{j}(x, a, b) & +\sum_{q=1}^{p} \mathcal{F}^{q}(a, b) \Pi_{a^{q}}^{j}(x, a, b) \\
& +\sum_{l=1}^{m} \mathcal{G}^{l}(a, b) \Pi_{b^{l}}^{j}(x, a, b),
\end{aligned}
$$

for $j=1, \ldots, m$ and for $(x, y, a, b) \in \mathcal{M}$. In fact, after replacing the variable $y$ by $\Pi(x, a, b)$, these equations should be interpreted as power series identities in $\mathbb{K}\{x, a, b\}$.

Denote by $\Delta(x, a, b)$ the determinant of the (invertible) matrix $\left(\Pi_{b^{l}}^{j}(x, a, b)\right)_{1 \leqslant l, j \leqslant m}$ and by $D(x, a, b)$ its matrix of cofactors, so that $\Pi_{b}^{-1}=[\Delta]^{-1} D$. Hence we can solve $\mathcal{G}$ from (6.43):

$$
\left\{\begin{aligned}
\mathcal{G}(a, b) \equiv \frac{D(x, a, b)}{\Delta(x, a, b)}[ & \mathcal{Y}(x, \Pi(x, a, b))-\sum_{i=1}^{n} \mathcal{X}^{i}(x, \Pi(x, a, b)) \Pi_{x^{i}}(x, a, b)- \\
& \left.-\sum_{q=1}^{p} \mathcal{F}^{q}(a, b) \Pi_{a^{q}}(x, a, b)\right] .
\end{aligned}\right.
$$


Next, we aim to solve the $\mathcal{F}^{q}(a, b)$. Consequently, we gather all the other terms in the brackets as $\Psi_{0}\left(J_{x, a, b}^{1} \Pi, \mathcal{X}, \mathcal{Y}\right)$ :

$$
\mathcal{G}(a, b) \equiv \frac{D(x, a, b)}{\Delta(x, a, b)}\left[-\sum_{q=1}^{p} \mathcal{F}^{q}(a, b) \Pi_{a^{q}}(x, a, b)\right]+\frac{\Psi_{0}\left(J_{x, a, b}^{1} \Pi, \mathcal{X}, \mathcal{Y}\right)}{\Delta(x, a, b)} .
$$

Here, $\Psi_{0}$ is linear with respect to $(\mathcal{X}, \mathcal{Y})$, with polynomial coefficients of degree one in $J_{x, a, b}^{1} \Pi$.

Next, for $k=1, \ldots, n$, we differentiate this identity with respect to $x_{k}$. Then $\mathcal{G}(a, b)$ disappears and we chase the denominator $\Delta^{2}$ :

$$
\left\{\begin{aligned}
0 \equiv[\Delta D][ & \left.-\sum_{q=1}^{p} \mathcal{F}^{q}(a, b) \Pi_{a^{q} x^{k}}(x, a, b)\right]+ \\
& +\left[\Delta D_{x_{k}}-\Delta_{x_{k}} D\right]\left[-\sum_{q=1}^{p} \mathcal{F}^{q}(a, b) \Pi_{a^{q}}(x, a, b)\right]+ \\
& +\Psi_{k}\left(J_{x, a, b}^{2} \Pi, J_{x, y}^{1} \mathcal{X}, J_{x, y}^{1} \mathcal{Y}\right) .
\end{aligned}\right.
$$

The $\Psi_{k}$ are linear with respect to $\left(J_{x, y}^{1} \mathcal{X}, J_{x, y}^{1} \mathcal{Y}\right)$, with polynomial coefficients in $J_{x, a, b}^{2} \Pi$. Then we further differentiate with respect to $x$ and by induction, for every $\beta \in \mathbb{N}^{n}$, we get:

$$
\left\{\begin{aligned}
0 \equiv & {[\Delta D]\left[-\sum_{q=1}^{p} \mathcal{F}^{q}(a, b) \Pi_{a^{q} x^{\beta}}(x, a, b)\right]+} \\
& +\sum_{\left|\beta_{1}\right|<|\beta|} \mathrm{D}_{\beta, \beta_{1}}\left(J^{\left|\beta_{1}\right|+1} \Pi\right)\left[-\sum_{q=1}^{p} \mathcal{F}^{q}(a, b) \Pi_{a^{q} x^{\beta_{1}}}(x, a, b)\right]+ \\
& +\Psi_{\beta}\left(J_{x, a, b}^{|\beta|+1} \Pi, J_{x, y}^{|\beta|} \mathcal{X}, J_{x, y}^{|\beta|} \mathcal{Y}\right),
\end{aligned}\right.
$$

where the expressions $D_{\beta, \beta_{1}}$ are certain $m \times m$ matrices with polynomial coefficients in the jet $J_{x, a, b}^{\left|\beta_{1}\right|+1} \Pi$, and where the terms $\Psi_{\beta}\left(J_{x, a, b}^{|\beta|+1} \Pi, J_{x, y}^{|\beta|} \mathcal{X}, J_{x, y}^{|\beta|} \mathcal{Y}\right)$ are linear with respect to $\left(J_{x, y}^{|\beta|} \mathcal{X}, J_{x, y}^{|\beta|} \mathcal{Y}\right)$, with polynomial coefficients in $J_{x, a, b}^{|\beta|+1} \Pi$.

Writing these identity for $(j, \beta)=(j(q), \beta(q)), q=1, \ldots, p$, reminding $\max _{1 \leqslant q \leqslant p}|\beta(q)|=\kappa$, it follows from the assumption of solvability with respect to the parameters (a boring technical check is needed) that we may solve

$$
\mathcal{F}^{q}(a, b) \equiv \Phi^{q}\left(J_{x, a, b}^{\kappa+1} \Pi(x, a, b), J_{x, y}^{\kappa} \mathcal{X}(x, \Pi(x, a, b)), J_{x, y}^{\kappa} \mathcal{Y}(x, \Pi(x, a, b))\right),
$$

for $q=1, \ldots, p$, where each local $\mathbb{K}$-analytic function $\Phi_{q}$ is linear with respect to $\left(J^{\kappa} \mathcal{X}, J^{\kappa} \mathcal{Y}\right)$ and rational with respect to $J^{\kappa+1} \Pi$, with denominator not vanishing at $(x, a, b):=(0,0,0)$.

Pursuing, we differentiate (6.48) with respect to $x^{l}$ for $l=1, \ldots, n$. Then $\mathcal{F}^{q}(a, b)$ disappears and we get:

$$
0 \equiv \Phi_{q, l}\left(J_{x, a, b}^{\kappa+2} \Pi(x, a, b), J_{x, y}^{\kappa+1} \mathcal{X}(x, \Pi(x, a, b)), J_{x, y}^{\kappa+1} \mathcal{Y}(x, \Pi(x, a, b))\right),
$$

for $1 \leqslant q \leqslant p$ and $1 \leqslant l \leqslant n$. In (6.46), we then replace the functions $\mathcal{F}^{q}$ by their values $\Phi^{q}$ :

$$
0 \equiv \Psi_{k, j}\left(J_{x, a, b}^{\kappa+1} \Pi(x, a, b), J_{x, y}^{\kappa} \mathcal{X}(x, \Pi(x, a, b)), J_{x, y}^{\kappa} \mathcal{Y}(x, \Pi(x, a, b))\right),
$$

for $1 \leqslant k \leqslant n$ and $1 \leqslant j \leqslant m$. Then we replace the variable $b$ by $\Pi^{*}(a, x, y)$ in the two obtained systems (6.49) and (6.50); taking account of the functional identity $y \equiv$ 
$\Pi\left(x, a, \Pi^{*}(a, x, y)\right)$ written in (6.41), we get

$$
\left\{\begin{array}{l}
0 \equiv \Phi_{q, l}\left(J_{x, a, b}^{\kappa+2} \Pi\left(x, a, \Pi^{*}(a, x, y)\right), J_{x, y}^{\kappa+1} \mathcal{X}(x, y), J_{x, y}^{\kappa+1} \mathcal{Y}(x, y)\right), \\
0 \equiv \Psi_{k, j}\left(J_{x, a, b}^{\kappa+1} \Pi\left(x, a, \Pi^{*}(a, x, y)\right), J_{x, y}^{\kappa} \mathcal{X}(x, y), J_{x, y}^{\kappa} \mathcal{Y}(x, y)\right) .
\end{array}\right.
$$

Finally, we develope these equations in power series with respect to $a$ :

$$
\left\{\begin{array}{l}
0 \equiv \sum_{\gamma \in \mathbb{N}^{p}} a^{\gamma} \Phi_{q, l, \gamma}\left(x, y, J_{x, y}^{\kappa+1} \mathcal{X}(x, y), J_{x, y}^{\kappa+1} \mathcal{Y}(x, y)\right) \\
0 \equiv \sum_{\gamma \in \mathbb{N}^{p}} a^{\gamma} \Psi_{k, j, \gamma}\left(x, y, J_{x, y}^{\kappa} \mathcal{X}(x, y), J_{x, y}^{\kappa} \mathcal{Y}(x, y)\right)
\end{array}\right.
$$

where the terms $\Phi_{q, l, \gamma}$ and $\Psi_{k, j, \gamma}$ are linear with respect to the jets of $\mathcal{X}, \mathcal{Y}$.

Proposition 6.53. A vector field (6.35) belongs to $\mathfrak{S} \mathfrak{Y M}(\mathcal{M})$ if and only if $\mathcal{X}^{i}, \mathcal{Y}^{j}$ satisfy the linear PDE system

$$
\left\{\begin{array}{l}
0 \equiv \Phi_{q, l, \gamma}\left(x, y, J_{x, y}^{\kappa+1} \mathcal{X}(x, y), J_{x, y}^{\kappa+1} \mathcal{Y}(x, y)\right) \\
0 \equiv \Pi_{k, j, \gamma}\left(x, y, J_{x, y}^{\kappa} \mathcal{X}(x, y), J_{x, y}^{\kappa} \mathcal{Y}(x, y)\right)
\end{array}\right.
$$

where $1 \leqslant q \leqslant p, 1 \leqslant l \leqslant n, 1 \leqslant k \leqslant n$ and $\gamma \in \mathbb{N}^{p}$. Then $\mathcal{F}^{q}$ defined by (6.48) and $\mathcal{G}^{j}$ defined by (6.45) are independent of $x$.

This provides a second algorithm, essentially equivalent to Sophus Lie's.

Example 6.55. For $y_{x x}(x)=F\left(x, y(x), y_{x}(x)\right)$, the first line of (6.54) is (the second one is redundant):

$$
\left\{\begin{aligned}
0 \equiv & \mathcal{X}\left[-\Pi_{x a} \Pi_{x x x} \Pi_{b}+\Pi_{a} \Pi_{x b} \Pi_{x x x}-\Pi_{x} \Pi_{x x a} \Pi_{x b}+\Pi_{x a} \Pi_{x} \Pi_{x x b}+\right. \\
& \left.+\Pi_{x x a} \Pi_{x x} \Pi_{b}-\Pi_{a} \Pi_{x x b} \Pi_{x x}\right]+ \\
& +\mathcal{Y}\left[-\Pi_{x a} \Pi_{x x b}+\Pi_{x x a} \Pi_{x b}\right]+ \\
& +\mathcal{X}_{x}\left[-2 \Pi_{x x} \Pi_{x a} \Pi_{b}+2 \Pi_{x x} \Pi_{a} \Pi_{x b}+\Pi_{x} \Pi_{b} \Pi_{x x a}-\Pi_{x} \Pi_{a} \Pi_{x x b}\right]+ \\
& +\mathcal{Y}_{x}\left[-\Pi_{b} \Pi_{x x a}+\Pi_{a} \Pi_{x x b}\right]+ \\
& +\mathcal{X}_{y}\left[-3 \Pi_{x} \Pi_{x x} \Pi_{x a} \Pi_{b}+3 \Pi_{x} \Pi_{a} \Pi_{x x} \Pi_{x b}+\left(\Pi_{x}\right)^{2} \Pi_{b} \Pi_{x x a}-\left(\Pi_{x}\right)^{2} \Pi_{a} \Pi_{x x b}\right]+ \\
& +\mathcal{Y}_{y}\left[\Pi_{x x} \Pi_{b} \Pi_{x a}-\Pi_{x x} \Pi_{a} \Pi_{x b}-\Pi_{x} \Pi_{b} \Pi_{x x a}+\Pi_{x} \Pi_{a} \Pi_{x x b}\right]+ \\
& +\mathcal{X}_{x x}\left[-\Pi_{x} \Pi_{b} \Pi_{x a}+\Pi_{x} \Pi_{a} \Pi_{x b}\right]+ \\
& +\mathcal{X}_{x y}\left[-2\left(\Pi_{x}\right)^{2} \Pi_{b} \Pi_{x a}+2\left(\Pi_{x}\right)^{2} \Pi_{a} \Pi_{x b}\right]+ \\
& +\mathcal{X}_{y^{2}}\left[-\left(\Pi_{x}\right)^{3} \Pi_{b} \Pi_{x a}+\left(\Pi_{x}\right)^{3} \Pi_{a} \Pi_{x b}\right]+ \\
& +\mathcal{Y}_{x x}\left[\Pi_{b} \Pi_{x a}-\Pi_{a} \Pi_{x b}\right]+ \\
& +\mathcal{Y}_{x y}\left[2 \Pi_{x} \Pi_{b} \Pi_{x a}-2 \Pi_{x} \Pi_{a} \Pi_{x b}\right]+ \\
& +\mathcal{Y}_{y^{2}}\left[\left(\Pi_{x}\right)^{2} \Pi_{b} \Pi_{x a}-\left(\Pi_{x}\right)^{2} \Pi_{a} \Pi_{x b}\right]
\end{aligned}\right.
$$

We observe the similarity with (4.19): the expression is linear in the partial derivatives of $\mathcal{X}, \mathcal{Y}$ of order $\leqslant 2$, but the coefficients in the equation above are more complicated. In fact, after dividing by $-\Pi_{b} \Pi_{x a}+\Pi_{a} \Pi_{x b}$, this equation coincides with (4.21), thanks to $\Pi_{x}=y_{1}$ and to the formulas (2.34) for $F_{x}, F_{y}, F_{y_{1}}$. 
6.57. Infinitesimal $C R$ automorphisms of generic submanifolds. If the system $(\mathcal{E})$ is associated to the complexification $\mathcal{M}=(M)^{c}$ of a generic $M \subset \mathbb{C}^{n+m}$ as in $\S 1.16$, then $a=(\bar{z})^{c}=\zeta, b=(\bar{w})^{c}=\xi$, and the vector field $\mathcal{L}^{*}$ associated to an infinitesimal Lie symmetry

$$
\mathcal{L}=\sum_{i=1}^{n} \mathcal{X}^{i}(z, w) \frac{\partial}{\partial z^{i}}+\sum_{j=1}^{m} \mathcal{Y}^{j}(z, w) \frac{\partial}{\partial w^{j}}
$$

of $(\mathcal{E})$ is simply the complexification $\underline{\mathcal{L}}$ of its conjugate $\overline{\mathcal{L}}$, namely

$$
\mathcal{L}^{*}=\underline{\mathcal{L}}=\sum_{i=1}^{n} \overline{\mathcal{X}}^{i}(\zeta, \xi) \frac{\partial}{\partial \zeta^{i}}+\sum_{j=1}^{m} \overline{\mathcal{Y}}^{j}(\zeta, \xi) \frac{\partial}{\partial \xi^{j}} .
$$

Then the sum $\mathcal{L}+\mathcal{L}$ is tangent to $\mathcal{M}$ and its flow stabilizes the two invariant foliations, obtained by intersecting $\mathcal{M}$ by $\{(z, w)=$ cst. $\}$ or by $\{(\zeta, \xi)=$ cst. $\}$. In [Me2005a, Me2005b], these two foliations, denoted $\mathcal{F}, \mathcal{F}$, are called (conjugate) Segre foliations, since its leaves are the complexifications of the (conjugate) classical Segre varieties ([Se1931, Pi1975, Pi1978, We1977, DW1980, BJT1985, DF1988, BER1999, Su2001, Su2002, Su2003, GM2003a]) associated to $M$, viewed in its ambient space $\mathbb{C}^{n+m}$. The next definition is also classical ([Be1979, Lo1981, EKV1985, Kr1987, KV1987, Be1988, Vi1990, St1996, Be1997, BER1999, Lo2002, FK2005a, FK2005b]):

Definition 6.60. By $\mathfrak{h o l}(M)$ is meant the Lie algebra of local holomorphic vector fields $\mathcal{L}=\sum_{i=1}^{n} \mathcal{X}^{i}(z, w) \frac{\partial}{\partial z^{i}}+\sum_{j=1}^{m} \mathcal{Y}^{j}(z, w) \frac{\partial}{\partial w^{j}}$ whose real flow $\exp (t \mathcal{L})(z, w)$ induces one-parameter families of local biholomorphic transformations of $\mathbb{C}^{n+m}$ stabilizing $M$. Equivalently,

$$
2 \operatorname{Re} \mathcal{L}=\mathcal{L}+\overline{\mathcal{L}}
$$

is tangent to $M$. Again equivalently, $\mathcal{L}+\underline{\mathcal{L}}$ is tangent to $\mathcal{M}=M^{c}$.

Then obviously $\mathfrak{h o r}(M)$ is a real Lie algebra.

Theorem 6.62. ([Ca1932a, BER1999, GM2004]) The complexification $\mathfrak{h o l}(M) \otimes \mathbb{C}$ identifies with $\mathfrak{S} \mathfrak{Y M}\left(\mathcal{E}\left(M^{c}\right)\right)$. Furthermore, if $M$ is finitely nondegenerate and minimal at the origin, both are finite-dimensional and $\mathfrak{h o l}(M)$ is totally real in $\mathfrak{S Y M M}\left(\mathcal{E}\left(M^{c}\right)\right)$.

The minimality assumption is sometimes presented by saying that the Lie algebra generated by $T^{c} M$ generates $T M$ at the origin ([BER1999]). However, it is more natural to proceed with the fundamental pair of foliations associated to $\mathcal{M}$ ([Me2001, GM2004, Me2005a, Me2005b]). Anticipating Sections 10 and 11 to which the reader is referred, we set.

Definition 6.63. A real analytic generic submanifold $M \subset \mathbb{C}^{n+m}$ is minimal at one of its points $p$ if the fundamental pair of foliations of its complexification $\mathcal{M}$ is covering at $p$ (Definition 10.17).

Further informations may be found in Section 10. We conclude by formulating applications of Theorems 5.13 and 5.24.

Corollary 6.64. The bound $\operatorname{dim} \mathfrak{h o l}(M) \leqslant 8$ for a Levi nondegenerate hypersurface $M \subset \mathbb{C}^{2}$ is attained if and only if it is locally biholomorphic to the sphere $S^{3} \subset \mathbb{C}^{2}$.

Corollary 6.65. The bound $\operatorname{dim} \mathfrak{h o l}(M) \leqslant n^{2}+4 n+3$ for a Levi nondegenerate hypersurface $M \subset \mathbb{C}^{n+1}$ is attained if and only if it is locally biholomorphic to the sphere $S^{2 n+1} \subset \mathbb{C}^{n+1}$. 


\section{$\S 7$. EQUIVALENCE PROBLEMS AND NORMAL FORMS}

7.1. Equivalences of submanifolds of solutions. As in $\S 3.1$, let $(\mathcal{E})$ and $\left(\mathcal{E}^{\prime}\right)$ be two PDE systems and assume that $\varphi$ transforms $(\mathcal{E})$ to $\left(\mathcal{E}^{\prime}\right)$. Defining $A^{\prime}$ similarly as $A$, it follows that

$$
\mathrm{A}^{\prime-1} \circ \Phi_{\mathcal{E}, \mathcal{E}^{\prime}} \circ \mathrm{A}(x, a, b) \equiv(\theta(x, a, b), f(a, b), g(a, b))=:\left(x^{\prime}, a^{\prime}, b^{\prime}\right)
$$

transforms $\mathrm{F}_{\mathrm{v}}$ to $\mathrm{F}_{\mathrm{v}}^{\prime}$, hence induces a map $(a, b) \mapsto\left(a^{\prime}, b^{\prime}\right)$. The arguments of Section 6 apply here with minor modifications to provide two fundamental lemmas.

Lemma 7.3. Every equivalence $(x, y) \mapsto\left(x^{\prime}, y^{\prime}\right)$ between to PDE systems $(\mathcal{E})$ and $\left(\mathcal{E}^{\prime}\right)$ comes with an associated transformation $(a, b) \mapsto\left(a^{\prime}, b^{\prime}\right)$ of the parameter spaces such that

$$
(x, y, a, b) \longmapsto\left(x^{\prime}, y^{\prime}, a^{\prime}, b^{\prime}\right)
$$

is an equivalence between the associated submanifolds of solutions $\mathcal{M}_{(\mathcal{E})} \rightarrow \mathcal{M}_{\left(\mathcal{E}^{\prime}\right)}^{\prime}$.

Conversely, let $\mathcal{M}$ and $\mathcal{M}^{\prime}$ be two submanifolds of $\mathbb{K}_{x}^{n} \times \mathbb{K}_{y}^{m} \times \mathbb{K}_{a}^{p} \times \mathbb{K}_{b}^{m}$ and of $\mathbb{K}_{x^{\prime}}^{n} \times \mathbb{K}_{y^{\prime}}^{m} \times \mathbb{K}_{a^{\prime}}^{p} \times \mathbb{K}_{b^{\prime}}^{m}$ represented by $y=\Pi(x, a, b)$ and by $y^{\prime}=\Pi^{\prime}\left(x^{\prime}, a^{\prime}, b^{\prime}\right)$, in the same dimensions. Assume both are solvable with respect to the parameters.

Lemma 7.5. Every equivalence

$$
(x, y, a, b) \longmapsto(\varphi(x, y), h(a, b))
$$

between $\mathcal{M}$ and $\mathcal{M}^{\prime}$ belonging to $\mathrm{G}_{\mathrm{v}, \mathrm{p}}$ induces by projection the equivalence $(x, y) \mapsto$ $\varphi(x, y)$ between the associated PDE systems $\left(\mathcal{E}_{\mathcal{M}}\right)$ and $\left(\mathcal{E}_{\mathcal{M}^{\prime}}^{\prime}\right)$.

7.7. Classification problems. Consequently, classifying PDE systems under point transformations (Section 3) is equivalent to the following.

Equivalence problem 7.8. Find an algorithm to decide whether two given submanifolds (of solutions) $\mathcal{M}$ and $\mathcal{M}^{\prime}$ are equivalent through an element of $\mathrm{G}_{\mathrm{v}, \mathrm{p}}$.

Classification problem 7.9. Classify submanifolds (of solutions) $\mathcal{M}$, namely provide a complete list of all possible such equations, including their automorphism group $\operatorname{Aut}_{\mathrm{v}, \mathrm{p}}(\mathcal{M}) \subset \mathrm{G}_{\mathrm{v}, \mathrm{p}}$.

7.10. Partial normal forms. Both problems above are of high complexity. At least as a preliminary step, it is useful to try to simplify somehow the defining equations of $\mathcal{M}$, by appropriate changes of coordinates belonging to $G_{v, p}$. To begin with, the next lemma holds for $\mathcal{M}$ defined by $y=\Pi(x, a, b)$ with the only assumption that $b \mapsto \Pi(0,0, b)$ has rank $m$ at $b=0$.

Lemma 7.11. ([CM1974, BER1999, Me2005a], [*]) In coordinates $x^{\prime}=\left(x^{1}, \ldots, x^{\prime n}\right)$ and $y^{\prime}=\left(y^{\prime 1}, \ldots, y^{\prime m}\right)$ an arbitrary submanifold $\mathcal{M}^{\prime}$ defined by $y^{\prime}=\Pi^{\prime}\left(x^{\prime}, a^{\prime}, b^{\prime}\right)$ or dually by $b^{\prime}=\Pi^{\prime *}\left(a^{\prime}, x^{\prime}, y^{\prime}\right)$ is equivalent to

$$
y=\Pi(x, a, b) \quad \text { or dually to } \quad b=\Pi^{*}(a, x, y)
$$

with

$$
\Pi(0, a, b) \equiv \Pi(x, 0, b) \equiv b \quad \text { or dually } \quad \Pi^{*}(0, x, y) \equiv \Pi^{*}(a, 0, y) \equiv y,
$$

namely $\Pi=b+\mathrm{O}(x a)$ and $\Pi^{*}=y+\mathrm{O}(a x)$. 
Proof. We develope

$$
y^{\prime}=\Pi^{\prime}\left(0, a^{\prime}, b^{\prime}\right)+\Lambda^{\prime}\left(x^{\prime}\right)+\mathrm{O}\left(x^{\prime} a^{\prime}\right) .
$$

Since $b^{\prime} \mapsto \Pi^{\prime}\left(0, a^{\prime}, b^{\prime}\right)$ has rank $m$ at $b^{\prime}=0$, the coordinate change

$$
b^{\prime \prime}:=\Pi^{\prime}\left(0, a^{\prime}, b^{\prime}\right), \quad a^{\prime \prime}:=a^{\prime}, \quad x^{\prime \prime}:=x^{\prime}, \quad y^{\prime \prime}:=y^{\prime},
$$

transforms $\mathcal{M}^{\prime}$ to $\mathcal{M}^{\prime \prime}$ defined by

$$
y^{\prime \prime}=\Pi^{\prime \prime}\left(x^{\prime \prime}, a^{\prime \prime}, b^{\prime \prime}\right):=b^{\prime \prime}+\Lambda^{\prime}\left(x^{\prime \prime}\right)+\mathrm{O}\left(x^{\prime \prime} a^{\prime \prime}\right) .
$$

Solving $b^{\prime \prime}$ by means of the implicit function theorem, we get

$$
b^{\prime \prime}=\Pi^{\prime \prime *}\left(a^{\prime \prime}, x^{\prime \prime}, y^{\prime \prime}\right)=y^{\prime \prime}-\Lambda^{\prime}\left(x^{\prime \prime}\right)+\mathrm{O}\left(a^{\prime \prime} x^{\prime \prime}\right),
$$

and it suffices to set $y:=y^{\prime \prime}-\Lambda^{\prime}\left(x^{\prime \prime}\right), x:=x^{\prime \prime}$ and $a:=a^{\prime \prime}, b:=b^{\prime \prime}$.

Taking account of solvability with respect to the parameters, finer normalizations holds.

Lemma 7.18. With $n=m=\kappa=1$, every submanifold of solutions $y^{\prime}=b^{\prime}+x^{\prime} a^{\prime}\left[1+\mathrm{O}_{1}\right]$ of $y_{x^{\prime} x^{\prime}}^{\prime}=F^{\prime}\left(x^{\prime}, y^{\prime}, y_{x^{\prime}}^{\prime}\right)$ is equivalent to

$$
y_{x x}=b+x a+\mathrm{O}\left(x^{2} a^{2}\right) .
$$

Proof. Writing $y^{\prime}=b^{\prime}+x^{\prime}\left[a^{\prime}+a^{\prime} \Lambda^{\prime}\left(a^{\prime}, b^{\prime}\right)+\mathrm{O}\left(x^{\prime} a^{\prime}\right)\right]$, where $\Lambda^{\prime}=\mathrm{O}_{1}$, we set $a^{\prime \prime}:=$ $a^{\prime}+a^{\prime} \Lambda^{\prime}\left(a^{\prime}, b^{\prime}\right), b^{\prime \prime}:=b^{\prime}, x^{\prime \prime}:=x^{\prime}, y^{\prime \prime}:=y^{\prime}$, whence $y^{\prime \prime}=b^{\prime \prime}+x^{\prime \prime}\left[a^{\prime \prime}+\mathrm{O}\left(x^{\prime \prime} a^{\prime \prime}\right)\right]$. Dually $b^{\prime \prime}=y^{\prime \prime}-a^{\prime \prime}\left[x^{\prime \prime}+x^{\prime \prime} x^{\prime \prime} \Lambda^{\prime \prime}\left(x^{\prime \prime}, y^{\prime \prime}\right)+\mathrm{O}\left(x^{\prime \prime} x^{\prime \prime} a^{\prime \prime}\right)\right]$, so we set $x:=x^{\prime \prime}+x^{\prime \prime} x^{\prime \prime} \Lambda^{\prime \prime}\left(x^{\prime \prime}, y^{\prime \prime}\right)$, $y:=y^{\prime \prime}, a:=a^{\prime \prime}, b:=b^{\prime \prime}$.

Corollary 7.20. Every second order ordinary differential equation $y_{x^{\prime} x^{\prime}}^{\prime}=F^{\prime}\left(x^{\prime}, y^{\prime}, y_{x^{\prime}}^{\prime}\right)$ is equivalent to

$$
y_{x x}=\left(y_{x}\right)^{2} \mathrm{R}\left(x, y, y_{x}\right) .
$$

7.22. Complete normal forms. The Moser theory of normal forms may be transferred with minor modifications to submanifolds of solutions associated to $\left(\mathcal{E}_{1}\right)$ and to $\left(\mathcal{E}_{2}\right)$.

Theorem 7.23. ([CM1974, Ja1990], [*]) A local $\mathbb{K}$-analytic submanifold of solutions associated to $\left(\mathcal{E}_{1}\right)$ :

$$
y^{\prime}=b^{\prime}+x^{\prime} a^{\prime}+\mathrm{O}_{3}=\sum_{k^{\prime} \geqslant 0} \sum_{l^{\prime} \geqslant 0} \Pi_{k^{\prime}, l^{\prime}}^{\prime}\left(b^{\prime}\right) x^{\prime k^{\prime}} a^{l^{\prime}}
$$

can be mapped, by a transformation $\left(x^{\prime}, y^{\prime}, a^{\prime}, b^{\prime}\right) \mapsto(x, y, a, b)$ belonging to $\mathrm{G}_{\mathrm{v}, \mathrm{p}}$, to a submanifold of solutions of the specific form

$$
y=b+x a+\Pi_{2,4}(b) x^{2} a^{4}+\Pi_{4,2}(b) a^{2} x^{4}+\sum_{k \geqslant 2} \sum_{l \geqslant 2} \sum_{k+l \geqslant 7} \Pi_{k, l}(b) x^{k} a^{l} .
$$

Solving $(a, b)$ from $y=\Pi$ and $y_{x}=\Pi_{x}$ with $\Pi$ as above, we deduce the following.

Corollary 7.26. Every $y_{x^{\prime} x^{\prime}}^{\prime}=F^{\prime}\left(x^{\prime}, y^{\prime}, y_{x^{\prime}}^{\prime}\right)$ is equivalent to

$$
\begin{aligned}
y_{x x}= & \left(y_{x}\right)^{2}\left[x^{2} F_{2,2}(y)+x^{3} \mathrm{r}(x, y)\right]+\left(y_{x}\right)^{4}\left[F_{0,4}(y)+x \mathrm{r}(x, y)\right]+ \\
& +\sum_{k \geqslant 0} \sum_{l \geqslant 0} \sum_{k+l \geqslant 5} F_{k, l}(y) x^{k}\left(y_{x}\right)^{l} .
\end{aligned}
$$

For the completely integrable system $\left(\mathcal{E}_{2}\right)$ having several dependent variables $\left(x^{1}, \ldots, x^{n}\right), n \geqslant 2$, we have the following. 
Theorem 7.28. ([CM1974], [*]) A local $\mathbb{K}$-analytic submanifold of solutions associated to $\left(\mathcal{E}_{2}\right)$ :

$$
y^{\prime}=b^{\prime}+\sum_{1 \leqslant k \leqslant n} x^{\prime k} a^{\prime k}+\mathrm{O}_{3}
$$

can be mapped, by a transformation $\left(x^{\prime}, y^{\prime}, a^{\prime}, b^{\prime}\right) \mapsto(x, y, a, b)$ belonging to $\mathrm{G}_{\mathrm{v}, \mathrm{p}}$, to a submanifold of solutions of the specific form:

$$
y=b+\sum_{1 \leqslant k \leqslant n} x^{k} a^{k}+\sum_{k \geqslant 2} \sum_{l \geqslant 2} \Pi_{k, l}(x, a, b)
$$

where

$$
\Pi_{k, l}(x, a, b):=\sum_{k_{1}+\cdots+k_{n}=k} \sum_{l_{1}+\cdots+l_{n}=l} \Pi_{k_{1}, \ldots, k_{n}, l_{1}, \ldots, l_{n}}(b)\left(x^{1}\right)^{k_{1}} \cdots\left(x^{n}\right)^{k_{n}}\left(a^{1}\right)^{l_{1}} \cdots\left(a^{n}\right)^{l_{n}}
$$

with the terms $\Pi_{2,2}, \Pi_{2,3}$ and $\Pi_{3,3}$ satisfying:

$$
0=\Delta \Pi_{2,2}=\Delta \Delta \Pi_{2,3}=\Delta \Delta \Pi_{3,2}=\Delta \Delta \Delta \Pi_{3,3},
$$

where

$$
\Delta:=\sum_{1 \leqslant k \leqslant n} \frac{\partial^{2}}{\partial x^{k} \partial a^{k}} .
$$

Exercise: solving $\left(a^{k}, b\right)$ from $y=\Pi$ and $y_{x^{l}}=\Pi_{x^{l}}$, with $\Pi$ as above, deduce a complete normal form for $\left(\mathcal{E}_{2}\right)$.

Open problem 7.34. Find complete normal forms for submanifolds of solutions associated to $\left(\mathcal{E}_{4}\right)$ and to $\left(\mathcal{E}_{5}\right)$.

\section{$\S 8$. STUdy OF TWO SPECIFIC EXAMPLES}

8.1. Study of the Lie symmetries of $\left(\mathcal{E}_{4}\right)$. Its submanifold of solutions possesses two equations:

$$
y^{1}=\Pi^{1}\left(x, a, b^{1}, b^{2}\right) \quad y^{2}=\Pi^{2}\left(x, a, b^{1}, b^{2}\right) .
$$

For instance, a generic submanifold $M \subset \mathbb{C}^{3}$ of $\mathrm{CR}$ dimension 1 and of codimension 3 has equations of such a form.

Assuming $\mathcal{V}_{\mathcal{S}}\left(\mathcal{E}_{4}\right)$ to be twin solvable and having covering submanifold of solutions (see Definition 10.17), it may be verified (for $M \subset \mathbb{C}^{3}$, see [Be1997]) that at a Zariskigeneric point, its equations are of the form:

$$
\begin{aligned}
& y^{1}=b^{1}+x a+\mathrm{O}\left(x^{2}\right)+\mathrm{O}\left(b^{1}\right)+\mathrm{O}\left(b^{2}\right), \\
& y^{2}=b^{2}+x a(x+a)+\mathrm{O}\left(x^{3}\right)+\mathrm{O}\left(b^{1}\right)+\mathrm{O}\left(b^{2}\right) .
\end{aligned}
$$

The model has zero remainders with associated system

$$
y_{1}^{2}=2 x y_{1}^{1}+\left(y_{1}^{1}\right)^{2}, \quad y_{2}^{1}=0,
$$

the third equation $y_{2}^{2}=2 y_{1}^{1}$ being obtained by differentiating the first.

We may put the submanifold in partial normal form. Proceeding as in [BES2005], some partial normalizations belonging to $G_{v, p}$ yield:

$$
\begin{aligned}
& y^{1}=b^{1}+a x+a^{2}\left[\Pi_{3,2}^{1}(b) x^{3}+\Pi_{4,2}^{1}(b) x^{4}+\cdots\right]+\mathrm{O}\left(a^{3} x^{2}\right), \\
& y^{2}=b^{2}+a\left[x^{2}+\Pi_{4,1}^{2}(b) x^{4}+\cdots\right]+a^{2}\left[x+\Pi_{3,2}^{2}(b) x^{3}+\cdots\right]+\mathrm{O}\left(a^{3} x^{2}\right) .
\end{aligned}
$$


Redifferentiating, we get an appropriate, partially normalized system $\left(\mathcal{E}_{4}\right)$ :

(8.6)

$$
\left\{\begin{array}{l}
y_{1}^{2}=y_{1}^{1}\left(2 x+\mathbf{g}^{1}\right)+\left(y_{1}^{1}\right)^{2}\left(1+\mathbf{g}^{2}\right)+\left(y_{1}^{1}\right)^{3} \mathbf{s}+\left(y_{1}^{1}\right)^{4} \mathbf{s}+\left(y_{1}^{1}\right)^{5} \mathbf{s}+\left(y_{1}^{1}\right)^{6} \mathbf{R} \\
y_{2}^{1}=\left(y_{1}^{1}\right)^{2} \mathbf{h}+\left(y_{1}^{1}\right)^{3} \mathbf{R} \\
y_{2}^{2}=y_{1}^{1}\left(2+\mathbf{g}_{x}^{1}\right)+\left(y_{1}^{1}\right)^{2}\left(\mathbf{g}_{x}^{2}+\left(2 x+\mathbf{g}^{1}\right) \mathbf{h}\right)+\left(y_{1}^{1}\right)^{3} \mathbf{r}+\left(y_{1}^{1}\right)^{4} \mathbf{r}+\left(y_{1}^{1}\right)^{5} \mathbf{r}+\left(y_{1}^{1}\right)^{6} \mathbf{R}
\end{array}\right.
$$

where, precisely:

- $\mathbf{g}^{1}, \mathbf{g}^{2}$ and $\mathbf{h}$ are functions of $\left(x, y^{1}, y^{2}\right)$ satisfying $\mathbf{g}^{j}=\mathrm{O}(x x)+\mathrm{O}\left(y^{1}\right)+\mathrm{O}\left(y^{2}\right)$, $j=1,2$ and $\mathbf{h}=\mathrm{O}(x)+\mathrm{O}\left(y^{1}\right)+\mathrm{O}\left(y^{2}\right)$;

- $\mathrm{r}$ and $\mathrm{s}$ are unspecified functions, varying in the context, of $\left(x, y^{1}, y^{2}\right)$ with $\mathrm{s}=$ $\mathrm{O}(x)+\mathrm{O}\left(y^{1}\right)+\mathrm{O}\left(y^{2}\right)$, but possibly $\mathrm{r}(0) \neq 0$;

- $\mathrm{R}$ is a remainder function of all the variables $\left(x, y^{1}, y^{2}, y_{1}^{1}\right)$ parametrizing $\Delta_{\mathcal{E}_{4}}$.

Letting $\mathcal{L}=\mathcal{X} \frac{\partial}{\partial x}+\mathcal{Y}^{1} \frac{\partial}{\partial y^{1}}+\mathcal{Y}^{2} \frac{\partial}{\partial y^{2}}$ be a candidate infinitesimal Lie symmetry and applying $\mathcal{L}^{(2)}=\mathcal{L}+\mathbf{Y}_{1}^{1} \frac{\partial}{\partial y_{1}^{1}}+\mathbf{Y}_{1}^{2} \frac{\partial}{\partial y_{1}^{2}}+\mathbf{Y}_{2}^{1} \frac{\partial}{\partial y_{2}^{1}}+\mathbf{Y}_{2}^{2} \frac{\partial}{\partial y_{2}^{2}}$ to $\Delta_{\mathcal{E}_{4}}$, we obtain firstly, computing $\bmod \left(y_{1}^{1}\right)^{5}$ :

$$
\begin{aligned}
0 \equiv & -\mathbf{Y}_{1}^{2}+[\mathcal{X}]\left(y_{1}^{1}\left(2+\mathbf{g}_{x}^{1}\right)+\left(y_{1}^{1}\right)^{2} \mathbf{g}_{x}^{2}+\left(y_{1}^{1}\right)^{3} \mathbf{r}+\left(y_{1}^{1}\right)^{4} \mathbf{r}\right)+ \\
& +\left[\mathcal{Y}^{1}\right]\left(y_{1}^{1} \mathbf{r}+\left(y_{1}^{1}\right)^{2} \mathbf{r}+\left(y_{1}^{1}\right)^{3} \mathbf{r}+\left(y_{1}^{1}\right)^{4} \mathbf{r}\right)+ \\
& +\left[\mathcal{Y}^{2}\right]\left(y_{1}^{1} \mathbf{r}+\left(y_{1}^{1}\right)^{2} \mathbf{r}+\left(y_{1}^{1}\right)^{3} \mathbf{r}+\left(y_{1}^{1}\right)^{4} \mathbf{r}\right)+ \\
& +\mathbf{Y}_{1}^{1}\left(2 x+\mathbf{g}^{1}+y_{1}^{1}\left(2+2 \mathbf{g}^{2}\right)+\left(y_{1}^{1}\right)^{2} \mathbf{s}+\left(y_{1}^{1}\right)^{3} \mathbf{s}+\left(y_{1}^{1}\right)^{4} \mathbf{s}\right),
\end{aligned}
$$

and secondly, computing $\bmod \left(y_{1}^{1}\right)^{2}$ :

$$
0 \equiv-\mathbf{Y}_{2}^{1}+2 y_{1}^{1} \mathbf{Y}_{1}^{1} h
$$

The third Lie equation involving $\mathbf{Y}_{2}^{2}$ will be superfluous. Specializing (4.6)(II) to $m=2$, we get $\mathbf{Y}_{1}^{1}$ and $\mathbf{Y}_{1}^{2}$ :

$$
\begin{aligned}
& \mathbf{Y}_{1}^{1}=\mathcal{Y}_{x}^{1}+\left[\mathcal{Y}_{y^{1}}^{1}-\mathcal{X}_{x}\right] y_{1}^{1}+\left[\mathcal{Y}_{y^{2}}^{1}\right] y_{1}^{2}+\left[-\mathcal{X}_{y^{1}}\right]\left(y_{1}^{1}\right)^{2}+\left[-\mathcal{X}_{y^{2}}\right] y_{1}^{1} y_{1}^{2} \\
& \mathbf{Y}_{1}^{2}=\mathcal{Y}_{x}^{2}+\left[\mathcal{Y}_{y^{1}}^{2}\right] y_{1}^{1}+\left[\mathcal{Y}_{y^{2}}^{2}-\mathcal{X}_{x}\right] y_{1}^{2}+\left[-\mathcal{X}_{y^{1}}\right] y_{1}^{2} y_{1}^{1}+\left[-\mathcal{X}_{y^{2}}\right]\left(y_{1}^{2}\right)^{2}
\end{aligned}
$$

and also $\mathbf{Y}_{2}^{1}$ and $\mathbf{Y}_{2}^{2}$ (in fact superfluous):

(8.10)

$$
\begin{aligned}
\mathbf{Y}_{2}^{1}= & \mathcal{Y}_{x x}^{1}+\left[2 \mathcal{Y}_{x y^{1}}^{1}-\mathcal{X}_{x x}\right] y_{1}^{1}+\left[2 \mathcal{Y}_{x y^{2}}^{1}\right] y_{1}^{2}+\left[\mathcal{Y}_{y^{1} y^{1}}^{1}-2 \mathcal{X}_{x y^{1}}\right]\left(y_{1}^{1}\right)^{2}+ \\
& +\left[2 \mathcal{Y}_{y^{1} y^{2}}^{1}-2 \mathcal{X}_{x y^{2}}\right] y_{1}^{1} y_{1}^{2}+\left[\mathcal{Y}_{y^{2} y^{2}}^{1}\right]\left(y_{1}^{2}\right)^{2}+\left[-\mathcal{X}_{y^{1} y^{1}}\right]\left(y_{1}^{1}\right)^{3}+ \\
& +\left[-2 \mathcal{X}_{y^{1} y^{2}}\right]\left(y_{1}^{1}\right)^{2} y_{1}^{2}+\left[-\mathcal{X}_{y^{2} y^{2}}\right] y_{1}^{1}\left(y_{1}^{2}\right)^{2}+\left[\mathcal{Y}_{y^{1}}^{1}-2 \mathcal{X}_{x}\right] y_{2}^{1}+ \\
& +\left[\mathcal{Y}_{y^{2}}^{1}\right] y_{2}^{2}+\left[-3 \mathcal{X}_{y^{1}}\right] y_{1}^{1} y_{2}^{1}+\left[-\mathcal{X}_{y^{2}}\right] y_{1}^{1} y_{2}^{2}+\left[-2 \mathcal{X}_{y^{2}}\right] y_{1}^{2} y_{2}^{1}, \\
\mathbf{Y}_{2}^{2}= & \mathcal{Y}_{x x}^{2}+\left[2 \mathcal{Y}_{x y^{1}}^{2}\right] y_{1}^{1}+\left[2 \mathcal{Y}_{x y^{2}}^{2}-\mathcal{X}_{x x}\right] y_{1}^{2}+\left[\mathcal{Y}_{y^{1} y^{1}}^{2}\right]\left(y_{1}^{1}\right)^{2}+ \\
& +\left[2 \mathcal{Y}_{y^{1} y^{2}}^{2}-2 \mathcal{X}_{x y^{1}}\right] y_{1}^{1} y_{1}^{2}+\left[\mathcal{Y}_{y^{2} y^{2}}^{2}-2 \mathcal{X}_{x y^{2}}\right]\left(y_{1}^{2}\right)^{2}+\left[-\mathcal{X}_{y^{1} y^{1}}\right]\left(y_{1}^{1}\right)^{2} y_{1}^{2}+ \\
& +\left[-2 \mathcal{X}_{y^{1} y^{2}}\right] y_{1}^{1}\left(y_{1}^{2}\right)^{2}+\left[-\mathcal{X}_{y^{2} y^{2}}\right]\left(y_{1}^{2}\right)^{3}+\left[\mathcal{Y}_{y^{1}}^{2}\right] y_{2}^{1}+ \\
& +\left[\mathcal{Y}_{y^{2}}^{2}-2 \mathcal{X}_{x}\right] y_{2}^{2}+\left[-2 \mathcal{X}_{y^{1}}\right] y_{1}^{1} y_{2}^{2}+\left[-\mathcal{X}_{y^{1}}\right] y_{1}^{2} y_{2}^{1}+\left[-3 \mathcal{X}_{y^{2}}\right] y_{1}^{2} y_{2}^{2}
\end{aligned}
$$


Inserting $\mathbf{Y}_{1}^{2}$ and $\mathbf{Y}_{1}^{1}$ in the first Lie equation (8.7) in which $y_{1}^{2}$ is replaced by the value $(8.6)_{1}$ it has on $\Delta_{\mathcal{E}_{4}}$ and still computing $\bmod \left(y_{1}^{1}\right)^{5}$, we get, again with $r$, s being unspecified functions of $\left(x, y^{1}, y^{2}\right)$ with $\mathrm{s}(0)=0$ :

(8.11)

$$
\begin{aligned}
0 \equiv & -\mathcal{Y}_{x}^{2}+\left[-\mathcal{Y}_{y^{1}}^{2}\right] y_{1}^{1}+ \\
& +\left[-\mathcal{Y}_{y^{2}}^{2}+\mathcal{X}_{x}\right]\left(y_{1}^{1}\left(2 x+\mathbf{g}^{1}\right)+\left(y_{1}^{1}\right)^{2}\left(1+\mathbf{g}^{2}\right)+\left(y_{1}^{1}\right)^{3} \mathbf{s}+\left(y_{1}^{1}\right)^{4} \mathbf{s}\right)+ \\
& +\left[\mathcal{X}_{y^{1}}\right]\left(\left(y_{1}^{1}\right)^{2}\left(2 x+\mathbf{g}^{1}\right)+\left(y_{1}^{1}\right)^{3}\left(1+\mathbf{g}^{2}\right)+\left(y_{1}^{1}\right)^{4} \mathbf{s}\right)+ \\
& +\left[\mathcal{X}_{y^{2}}\right]\left(\left(y_{1}^{1}\right)^{2}\left[2 x+\mathbf{g}^{1}\right]^{2}+\left(y_{1}^{1}\right)^{3}\left(4 x+2 \mathbf{g}^{1}\right)\left(1+\mathbf{g}^{2}\right)+\left(y_{1}^{1}\right)^{4}(1+\mathbf{s})\right)+ \\
& +[\mathcal{X}]\left(y_{1}^{1}\left(2+\mathbf{g}_{x}^{1}\right)+\left(y_{1}^{1}\right)^{2} \mathbf{g}_{x}^{2}+\left(y_{1}^{1}\right)^{3} \mathbf{r}+\left(y_{1}^{1}\right)^{4} \mathbf{r}\right)+ \\
& +\left[\mathcal{Y}^{1}\right]\left(y_{1}^{1} \mathbf{r}+\left(y_{1}^{1}\right)^{2} \mathbf{r}+\left(y_{1}^{1}\right)^{3} \mathbf{r}+\left(y_{1}^{1}\right)^{4} \mathbf{r}\right)+ \\
& +\left[\mathcal{Y}^{2}\right]\left(y_{1}^{1} \mathbf{r}+\left(y_{1}^{1}\right)^{2} \mathbf{r}+\left(y_{1}^{1}\right)^{3} \mathbf{r}+\left(y_{1}^{1}\right)^{4} \mathbf{r}\right)+ \\
& +\left[\mathcal{Y}_{x}^{1}\right]\left(2 x+\mathbf{g}^{1}+y_{1}^{1}\left(2+2 \mathbf{g}^{2}\right)+\left(y_{1}^{1}\right)^{2} \mathbf{s}+\left(y_{1}^{1}\right)^{3} \mathbf{s}+\left(y_{1}^{1}\right)^{4} \mathbf{s}\right)+ \\
& +\left[\mathcal{Y}_{y^{1}}^{1}-\mathcal{X}_{x}\right]\left(y_{1}^{1}\left(2 x+\mathbf{g}^{1}\right)+\left(y_{1}^{1}\right)^{2}\left(2+2 \mathbf{g}^{2}\right)+\left(y_{1}^{1}\right)^{3} \mathbf{s}+\left(y_{1}^{1}\right)^{4} \mathbf{s}\right)+ \\
& +\left[\mathcal{Y}_{y^{2}}^{1}\right]\left(y_{1}^{1}\left[2 x+\mathbf{g}^{1}\right]^{2}+\left(y_{1}^{1}\right)^{2}\left(2 x+\mathbf{g}^{1}\right)\left(3+3 \mathbf{g}^{2}\right)+\left(y_{1}^{1}\right)^{3}(2+\mathbf{s})+\left(y_{1}^{1}\right)^{4} \mathbf{s}\right)+ \\
& +\left[-\mathcal{X}_{y^{1}}\right]\left(\left(y_{1}^{1}\right)^{2}\left(2 x+\mathbf{g}^{1}\right)+\left(y_{1}^{1}\right)^{3}\left(2+2 \mathbf{g}^{2}\right)+\left(y_{1}^{1}\right)^{4} \mathbf{s}\right)+ \\
& +\left[-\mathcal{X}_{y^{2}}\right]\left(\left(y_{1}^{1}\right)^{2}\left[2 x+\mathbf{g}^{1}\right]^{2}+\left(y_{1}^{1}\right)^{3}\left(2 x+\mathbf{g}^{1}\right)\left(3+3 \mathbf{g}^{2}\right)+\left(y_{1}^{1}\right)^{4}(2+\mathbf{s})\right) .
\end{aligned}
$$

Collecting the coefficients of the monomials cst., $y_{1}^{1},\left(y_{1}^{1}\right)^{2},\left(y_{1}^{1}\right)^{3},\left(y_{1}^{1}\right)^{4}$, we get, after slight simplification (in the coefficient of $\left(y_{1}^{1}\right)^{2}$, the term $\left(2 x+\mathbf{g}^{1}\right) \mathcal{X}_{x}$ annihilates with its opposite; in the coefficient of $\left(y_{1}^{1}\right)^{3}$, two pairs annihilate and then, we divide by $\left[1+\mathbf{g}^{2}\right]$ ) a system of five linear PDE's:

$$
\begin{aligned}
& 0=-\mathcal{Y}_{x}^{2}+\left(2 x+\mathbf{g}^{1}\right) \mathcal{Y}_{x}^{1} \\
& 0=-\mathcal{Y}_{y^{1}}^{2}-\left(2 x+\mathbf{g}^{1}\right) \mathcal{Y}_{y^{2}}^{2}+\left(2+\mathbf{g}_{x}^{1}\right) \mathcal{X}+\mathbf{r} \mathcal{Y}^{1}+\mathbf{r} \mathcal{Y}^{2}+ \\
& +\left(2+2 \mathbf{g}^{2}\right) \mathcal{Y}_{x}^{1}+\left(2 x+\mathbf{g}^{1}\right) \mathcal{Y}_{y^{1}}^{1}+\left[2 x+\mathbf{g}^{1}\right]^{2} \mathcal{Y}_{y^{2}}^{1}, \\
& 0=-\mathcal{Y}_{y^{2}}^{2}+\mathcal{X}_{x}+\mathbf{g}_{x}^{2}\left[1+\mathbf{g}^{2}\right]^{-1} \mathcal{X}+\mathbf{r} \mathcal{Y}^{1}+\mathbf{r} \mathcal{Y}^{2}+ \\
& +\mathrm{s} \mathcal{Y}_{x}^{1}+2 \mathcal{Y}_{y^{1}}^{1}-2 \mathcal{X}_{x}+\left(6 x+3 \mathrm{~g}^{2}\right) \mathcal{Y}_{y^{2}}^{1} \\
& 0=\mathrm{s} \mathcal{Y}_{y^{2}}^{2}+\mathrm{s} \mathcal{X}_{x}+\left(1+\mathbf{g}^{2}\right) \mathcal{X}_{y^{1}}+\left(2 x+\mathbf{g}^{1}\right)\left(2+2 \mathbf{g}^{2}\right) \mathcal{X}_{y^{2}}+ \\
& +\mathrm{r} \mathcal{X}+\mathrm{r} \mathcal{Y}^{1}+\mathrm{r} \mathcal{Y}^{2}+\mathrm{s} \mathcal{Y}_{x}^{1}+\mathrm{s} \mathcal{Y}_{y^{1}}^{1}+\mathrm{s} \mathcal{X}_{x}+(2+\mathrm{s}) \mathcal{Y}_{y^{2}}^{1}- \\
& -\left(2+2 \mathbf{g}^{2}\right) \mathcal{X}_{y^{1}}-\left(2 x+\mathbf{g}^{1}\right)\left(3+3 \mathbf{g}^{2}\right) \mathcal{X}_{y^{2}}, \\
& 0=\mathrm{s} \mathcal{Y}_{y^{2}}^{2}+\mathrm{s} \mathcal{X}_{x}+\mathrm{s} \mathcal{X}_{y^{1}}+(1+\mathrm{s}) \mathcal{X}_{y^{2}}+\mathrm{r} \mathcal{X}+\mathrm{r} \mathcal{Y}^{1}+\mathrm{r} \mathcal{Y}^{2}+\mathrm{s} \mathcal{Y}_{x}^{1}+\mathrm{s} \mathcal{Y}_{y^{1}}^{1}+ \\
& +\mathrm{s} \mathcal{X}_{x}+\mathrm{s} \mathcal{Y}_{y^{2}}^{1}+\mathrm{s} \mathcal{X}_{y^{1}}-(2+\mathrm{s}) \mathcal{X}_{y^{2}}
\end{aligned}
$$

We then simplify the remainders using $s+s=s, r+s=r$ and $r+r=r$; we divide $(8.12)_{5}$ by $(1+s)$; we replace $\mathcal{X}_{y^{2}}$ obtained from $(8.12)_{5}$ in $(8.12)_{4}$; we divide $(8.12)_{4}$ by 
$\left(1+\mathbf{g}^{2}\right)$; we then solve $\mathcal{X}_{y^{1}}$ from $(8.12)_{4}$ and finally we insert it in (8.12) $)_{5}$; we get:

$$
\begin{aligned}
& 0=-\mathcal{Y}_{x}^{2}+\left(2 x+\mathbf{g}^{1}\right) \mathcal{Y}_{x}^{1}, \\
& 0=-\mathcal{Y}_{y^{1}}^{2}-\left(2 x+\mathbf{g}^{1}\right) \mathcal{Y}_{y^{2}}^{2}+\left(2+\mathbf{g}_{x}^{1}\right) \mathcal{X}+\left(2+2 \mathbf{g}^{2}\right) \mathcal{Y}_{x}^{1}+\left(2 x+\mathbf{g}^{1}\right) \mathcal{Y}_{y^{1}}^{1}+ \\
& +\left[2 x+\mathbf{g}^{1}\right]^{2} \mathcal{Y}_{y^{2}}^{1}+\mathbf{r} \mathcal{Y}^{1}+\mathbf{r} \mathcal{Y}^{2} \text {, } \\
& 0=-\mathcal{Y}_{y^{2}}^{2}-\mathcal{X}_{x}+2 \mathcal{Y}_{y^{1}}^{1}+\left(6 x+3 \mathrm{~g}^{2}\right) \mathcal{Y}_{y^{2}}^{1}+\mathrm{r} \mathcal{Y}^{1}+\mathrm{r} \mathcal{Y}^{2}+\mathrm{s} \mathcal{Y}_{x}^{1}+ \\
& +\mathbf{g}_{x}^{2}\left[1+\mathbf{g}^{2}\right]^{-1} \mathcal{X} \\
& 0=-\mathcal{X}_{y^{1}}+(2+\mathrm{s}) \mathcal{Y}_{y^{2}}^{1}+\mathrm{r} \mathcal{X}+\mathrm{r} \mathcal{Y}^{1}+\mathrm{r} \mathcal{Y}^{2}+\mathrm{s} \mathcal{X}_{x}+\mathrm{s} \mathcal{Y}_{x}^{1}+\mathrm{s} \mathcal{Y}_{y^{1}}^{1}+\mathrm{s} \mathcal{Y}_{y^{2}}^{2}, \\
& 0=-\mathcal{X}_{y^{2}}+\mathrm{r} \mathcal{X}+\mathrm{r} \mathcal{Y}^{1}+\mathrm{r} \mathcal{Y}^{2}+\mathrm{s} \mathcal{X}_{x}+\mathrm{s} \mathcal{Y}_{x}^{1}+\mathrm{s} \mathcal{Y}_{y^{1}}^{1}+\mathrm{s} \mathcal{Y}_{y^{2}}^{1}+\mathrm{s} \mathcal{Y}_{y^{2}}^{2} \text {. }
\end{aligned}
$$

Similarly, developing the second equation (8.8) and computing $\bmod \left(y_{1}^{1}\right)^{2}$, we get:

$$
\begin{aligned}
0 \equiv-\mathcal{Y}_{x x}^{1} & +\left[-2 \mathcal{Y}_{x y^{1}}^{1}+\mathcal{X}_{x x}\right] y_{1}^{1}+\left[-\left(4 x+2 \mathbf{g}^{1}\right) \mathcal{Y}_{x y^{2}}-(2+\mathbf{h}) \mathcal{Y}_{y^{2}}^{1}\right]+ \\
& +\left[2 \mathbf{h} \mathcal{Y}_{x}^{1}\right] y_{1}^{1} .
\end{aligned}
$$

Collecting the coefficients of the monomials cst., $y_{1}^{1}$, we get two more linear PDE's:

$$
\begin{aligned}
& 0=-\mathcal{Y}_{x x}^{1}, \\
& 0=-2 \mathcal{Y}_{x y^{1}}^{1}+\mathcal{X}_{x x}-\left(4 x+2 \mathbf{g}^{1}\right) \mathcal{Y}_{x y^{2}}^{1}-(2+\mathbf{h}) \mathcal{Y}_{y^{2}}^{1}+2 \mathbf{h} \mathcal{Y}_{x}^{1} .
\end{aligned}
$$

Proposition 8.16. Setting as initial conditions the five specific differential coefficients

$$
\mathrm{P}:=\mathrm{P}\left(\mathcal{X}, \mathcal{Y}^{1}, \mathcal{Y}^{2}, \mathcal{Y}_{x}^{1}, \mathcal{X}_{x}\right)=\mathrm{r} \mathcal{X}+\mathrm{r} \mathcal{Y}^{1}+\mathrm{r} \mathcal{Y}^{2}+\mathrm{r} \mathcal{Y}_{x}^{1}+\mathrm{r} \mathcal{X}_{x}
$$

it follows by cross differentiations and by linear substitutions from the seven equations $(8.13)_{i}, i=1,2,3,4,5,(8.15)_{j}, j=1,2$, that $\mathcal{X}_{y^{1}}, \mathcal{X}_{y^{2}}, \mathcal{Y}_{y^{1}}^{1}, \mathcal{Y}_{y^{2}}^{1}, \mathcal{Y}_{x}^{2}, \mathcal{Y}_{y^{1}}^{2}, \mathcal{Y}_{y^{2}}^{2}$ and $\mathcal{X}_{x x}, \mathcal{X}_{x y^{1}}, \mathcal{X}_{x y^{2}}, \mathcal{Y}_{x x}^{1}, \mathcal{Y}_{x y^{1}}^{1}, \mathcal{Y}_{x y^{2}}^{1}$ are uniquely determined as linear combinations of $\left(\mathcal{X}, \mathcal{Y}^{1}, \mathcal{Y}^{2}, \mathcal{Y}_{x}^{1}, \mathcal{X}_{x}\right)$, namely:

$$
\left\{\begin{array}{ccccc} 
& \mathcal{Y}_{x}^{2} \stackrel{1}{=} \mathrm{P}, & \mathcal{X}_{x x} \stackrel{2}{=} \mathrm{P}, & \mathcal{Y}_{x x}^{1} \stackrel{3}{=} \mathrm{P} \\
\mathcal{X}_{y^{1}} \stackrel{4}{=} \mathrm{P}, & \mathcal{Y}_{y^{1}}^{1} \stackrel{5}{=} \mathrm{P}, & \mathcal{Y}_{y^{1}}^{2} \stackrel{6}{=} \mathrm{P}, & \mathcal{X}_{x y^{1}} \stackrel{7}{=} \mathrm{P}, & \mathcal{Y}_{x y^{1}}^{1} \stackrel{8}{=} \mathrm{P} \\
\mathcal{X}_{y^{2}} \stackrel{9}{=} \mathrm{P}, & \mathcal{Y}_{y^{2}}^{1} \stackrel{10}{=} \mathrm{P}, & \mathcal{Y}_{y^{2}}^{2} \stackrel{11}{=} \mathrm{P}, & \mathcal{X}_{x y^{2}} \stackrel{12}{=} \mathrm{P}, & \mathcal{Y}_{x y^{2}}^{1} \stackrel{13}{=} \mathrm{P}
\end{array}\right.
$$

Then the expressions $\mathrm{P}$ are stable under differentiation:

$$
\begin{aligned}
\mathrm{P}_{x} & =\mathrm{P}+\mathrm{r} \mathcal{Y}_{x}^{2}+\mathrm{r} \mathcal{Y}_{x x}^{1}+\mathrm{r} \mathcal{X}_{x x}=\mathrm{P}, \\
\mathrm{P}_{y^{1}} & =\mathrm{P}+\mathrm{r} \mathcal{X}_{y^{1}}+\mathrm{r} \mathcal{Y}_{y^{1}}^{1}+\mathrm{r} \mathcal{Y}_{y^{1}}^{2}+\mathrm{r} \mathcal{Y}_{x y^{1}}^{1}+\mathrm{r} \mathcal{X}_{x y^{1}}=\mathrm{P}, \\
\mathrm{P}_{y^{2}} & =\mathrm{P}+\mathrm{r} \mathcal{X}_{y^{2}}+\mathrm{r} \mathcal{Y}_{y^{2}}^{1}+\mathrm{r} \mathcal{Y}_{y^{2}}^{2}+\mathrm{r} \mathcal{Y}_{x y^{2}}^{1}+\mathrm{r} \mathcal{X}_{x y^{2}}=\mathrm{P},
\end{aligned}
$$

and moreover, all other, higher order partial derivatives of $\mathcal{X}$, of $\mathcal{Y}^{1}$ and of $\mathcal{Y}^{2}$ may be expressed as $\mathrm{P}\left(\mathcal{X}, \mathcal{Y}^{1}, \mathcal{Y}^{2}, \mathcal{Y}_{x}^{1}, \mathcal{X}_{x}\right)$.

Corollary 8.20. An infinitesimal Lie symmetry of $\left(\mathcal{E}_{4}\right)$ is uniquely determined by the five initial Taylor coefficients

$$
\mathcal{X}(0), \mathcal{Y}^{1}(0), \mathcal{Y}^{2}(0), \mathcal{Y}_{x}^{1}(0), \mathcal{X}_{x}(0)
$$

Proof of the proposition. We notice that $(8.18)_{1}$ and $(8.18)_{3}$ are given for free by $(8.13)_{1}$ and by $(8.15)_{1}$. Differentiating $(8.13)_{3}$ with respect to $x$, we get:

$$
\begin{aligned}
0= & -\mathcal{Y}_{x y^{2}}-\mathcal{X}_{x x}+2 \mathcal{Y}_{x y^{1}}^{1}+\left(6+3 \mathbf{g}_{x}^{1}\right) \mathcal{Y}_{y^{2}}^{1}+\left(6 x+3 \mathbf{g}^{1}\right) \mathcal{Y}_{x y^{2}}^{1}+\mathbf{r} \mathcal{Y}^{1}+ \\
& +\mathrm{r} \mathcal{Y}_{x}^{1}+\mathrm{r} \mathcal{Y}^{2}+\mathrm{r} \mathcal{Y}_{x}^{2}+\mathrm{r} \mathcal{Y}_{x}^{1}+\mathrm{s} \mathcal{Y}_{x x}^{1}+\mathbf{r} \mathcal{X}+\mathrm{g}_{x}^{2}\left[1+\mathbf{g}^{2}\right]^{-1} \mathcal{X}_{x}
\end{aligned}
$$


By $(8.15)_{1}, \mathrm{~s} \mathcal{Y}_{x x}^{1}$ vanishes. We replace $\mathcal{Y}_{x}^{2}$ thanks to $(8.13)_{1}$. Differentiating $(8.13)_{1}$ with respect to $y^{2}$, we may substract $0=-\mathcal{Y}_{x y^{2}}+\left(2 x+\mathbf{g}^{1}\right) \mathcal{Y}_{x y^{2}}^{1}+\mathbf{r} \mathcal{Y}_{x}^{1}$. We get:

$$
\begin{aligned}
0= & -\mathcal{X}_{x x}+2 \mathcal{Y}_{x y^{1}}^{1}+\left(4 x+2 \mathbf{g}^{1}\right) \mathcal{Y}_{x y^{2}}^{1}+ \\
& +\left(6+3 \mathbf{g}_{x}^{1}\right) \mathcal{Y}_{y^{2}}^{1}+\mathbf{r} \mathcal{X}+\mathbf{r} \mathcal{Y}^{1}+\mathbf{r} \mathcal{Y}^{2}+\mathbf{r} \mathcal{Y}_{x}^{1}+\mathbf{g}_{x}^{2}\left[1+\mathbf{g}^{2}\right]^{-1} \mathcal{X}_{x}
\end{aligned}
$$

By means of $(8.15)_{2}$, we replace the first three terms and then solve $\mathcal{Y}_{y^{2}}^{1}$ :

$$
\mathcal{Y}_{y^{2}}^{1}=\mathbf{r} \mathcal{X}+\mathbf{r} \mathcal{Y}^{1}+\mathbf{r} \mathcal{Y}^{2}+\mathbf{r} \mathcal{Y}_{x}^{1}+\mathbf{k}^{*} \mathcal{X}_{x}
$$

introducing a notation for a new function that should be recorded:

$$
\mathbf{k}^{*}:=\mathbf{g}_{x}^{2}\left[1+\mathbf{g}^{2}\right]^{-1}\left[4+3 \mathbf{g}_{x}^{1}-\mathbf{h}\right]^{-1} \text {. }
$$

This is $(8.18)_{10}$. Next, we differentiate the obtained equation with respect to $x$, getting:

$$
\mathcal{Y}_{x y^{2}}^{1}=\mathrm{r} \mathcal{X}+\mathrm{r} \mathcal{Y}^{1}+\mathrm{r} \mathcal{Y}^{2}+\mathrm{r} \mathcal{Y}_{x}^{1}+\mathbf{r} \mathcal{X}_{x}+\mathbf{k}^{*} \mathcal{X}_{x x}
$$

This is $(8.18)_{13}$. We replace the obtained value of $\mathcal{Y}_{y^{2}}^{1}$ in $(8.13)_{2},(8.13)_{3},(8.15)_{2}$ and the obtained value of $\mathcal{Y}_{x y^{2}}^{1}$ in $(8.15)_{2}$. This yields a new, simpler system of seven equations:

$$
\begin{aligned}
0= & -\mathcal{Y}_{x}^{2}+\left(2 x+\mathrm{g}^{1}\right) \mathcal{Y}_{x}^{1} \\
0= & -\mathcal{Y}_{y^{1}}^{2}-\left(2 x+\mathrm{g}^{1}\right) \mathcal{Y}_{y^{2}}^{2}+\left(2+\mathrm{g}_{x}^{1}\right) \mathcal{X}+\left(2+2 \mathrm{~g}^{2}\right) \mathcal{Y}_{x}^{1}+\left(2 x+\mathrm{g}^{1}\right) \mathcal{Y}_{y^{1}}^{1}+ \\
& +\mathrm{s} \mathcal{X}+\mathrm{r} \mathcal{Y}^{1}+\mathrm{r} \mathcal{Y}^{2}+\mathrm{s} \mathcal{Y}_{x}^{1}+\mathrm{k}^{*}\left[2 x+\mathbf{g}^{1}\right]^{2} \mathcal{X}_{x}, \\
0= & -\mathcal{Y}_{y^{2}}^{2}-\mathcal{X}_{x}+2 \mathcal{Y}_{y^{1}}^{1}+\mathrm{s} \mathcal{X}+\mathrm{r} \mathcal{Y}^{1}+\mathrm{r} \mathcal{Y}^{2}+\mathrm{s} \mathcal{Y}_{x}^{1}+\mathrm{k}^{*}\left(6 x+3 \mathrm{~g}^{1}\right) \mathcal{X}_{x}, \\
0= & -\mathcal{X}_{y^{1}}+\mathrm{r} \mathcal{X}+\mathrm{r} \mathcal{Y}^{1}+\mathrm{r} \mathcal{Y}^{2}+\mathrm{s} \mathcal{Y}_{x}^{1}+\mathrm{s} \mathcal{X}_{x}+\mathrm{s} \mathcal{Y}_{y^{1}}^{1}+\mathrm{s} \mathcal{Y}_{y^{2}}^{2}, \\
0= & -\mathcal{X}_{y^{2}}+\mathrm{r} \mathcal{X}+\mathrm{r} \mathcal{Y}^{1}+\mathrm{r} \mathcal{Y}^{2}+\mathrm{s} \mathcal{Y}_{x}^{1}+\mathrm{s} \mathcal{X}_{x}+\mathrm{s} \mathcal{Y}_{y^{1}}^{1}+\mathrm{s} \mathcal{Y}_{y^{2}}^{2}, \\
0= & -\mathcal{Y}_{x x}^{1}, \\
0= & -2 \mathcal{Y}_{x y^{1}}^{1}+\mathcal{X}_{x x}\left(1-\mathrm{k}^{*}\left(4 x+2 \mathbf{g}^{1}\right)\right)+\mathrm{r} \mathcal{X}+\mathrm{r} \mathcal{Y}^{1}+\mathrm{r} \mathcal{Y}^{2}+\mathrm{r} \mathcal{Y}_{x}^{1} . \mathrm{s} \mathcal{X}_{x} .
\end{aligned}
$$

Restarting from this system, we differentiate $(8.27)_{3}$ with respect to $x$ :

$$
\begin{aligned}
0= & -\mathcal{Y}_{x y^{2}}^{2}-\mathcal{X}_{x x}+2 \mathcal{Y}_{x y^{1}}^{1}+\mathrm{r} \mathcal{X}+\mathrm{r} \mathcal{Y}^{1}+\mathrm{r} \mathcal{Y}^{2}+ \\
& +\mathrm{r} \mathcal{X}_{x}+\mathrm{r} \mathcal{Y}_{x}^{1}+\mathrm{r} \mathcal{Y}_{x}^{2}+\mathrm{s} \mathcal{Y}_{x x}^{1}+\mathrm{k}^{*}\left(6 x+3 \mathrm{~g}^{1}\right) \mathcal{X}_{x x} .
\end{aligned}
$$

We replace $\mathcal{Y}_{x}^{2}$, we erase $\mathcal{Y}_{x x}^{1}$ and we add $(8.27)_{7}$ :

$$
0=-\mathcal{Y}_{x y^{2}}^{2}+\mathrm{k}^{*}\left(2 x+\mathrm{g}^{1}\right) \mathcal{X}_{x x}+\mathrm{r} \mathcal{X}+\mathrm{r} \mathcal{Y}^{1}+\mathrm{r} \mathcal{Y}^{2}+\mathrm{r} \mathcal{Y}_{x}^{1}+\mathrm{r} \mathcal{X}_{x}
$$

We differentiate $(8.27)_{2}$ with respect to $x$ :

$$
\begin{aligned}
0= & -\mathcal{Y}_{x y^{1}}^{2}-\left(2+\mathrm{g}_{x}^{1}\right) \mathcal{Y}_{y^{2}}^{2}-\left(2 x+\mathrm{g}^{1}\right) \mathcal{Y}_{x y^{2}}^{2}+\mathrm{r} \mathcal{X}+\left(2+\mathrm{g}_{x}^{1}\right) \mathcal{X}_{x}+ \\
& +\mathrm{s} \mathcal{Y}_{x}^{1}+\left(2+2 \mathbf{g}^{2}\right) \mathcal{Y}_{x x}^{1}+\left(2+\mathbf{g}_{x}^{1}\right) \mathcal{Y}_{y^{1}}^{1}+\left(2 x+\mathbf{g}^{1}\right) \mathcal{Y}_{x y^{1}}^{1}+\mathbf{r} \mathcal{X}_{x}+ \\
& +\mathrm{s} \mathcal{X}_{x}+\mathbf{r} \mathcal{Y}^{1}+\mathbf{r} \mathcal{Y}_{x}^{1}+\mathbf{r} \mathcal{Y}^{2}+\mathbf{r} \mathcal{Y}_{x}^{2}+\mathbf{r} \mathcal{Y}_{x}^{1}+\mathbf{s} \mathcal{Y}_{x x}^{1}+\mathbf{k}^{*}\left[2 x+\mathbf{g}^{1}\right]^{2} \mathcal{X}_{x x}
\end{aligned}
$$

Differentiating $(8.27)_{1}$ with respect to $y^{1}$, we may substract $0=-\mathcal{Y}_{x y^{1}}^{2}+\left(2 x+\mathbf{g}^{1}\right) \mathcal{Y}_{x y^{1}}^{1}+$ $\mathrm{r} \mathcal{Y}_{x}^{1}$; we replace $\mathcal{Y}_{x}^{2}$ and erase $\mathcal{Y}_{x x}^{1}$; we substract (8.29) multiplied by $\left(2 x+\mathbf{g}^{1}\right)$; we get:

$$
0=-\mathcal{Y}_{y^{2}}^{2}+(1+\mathrm{s}) \mathcal{X}_{x}+\mathcal{Y}_{y^{1}}^{1}+\mathrm{r} \mathcal{X}+\mathrm{r} \mathcal{Y}^{1}+\mathrm{r} \mathcal{Y}^{2}+\mathrm{r} \mathcal{Y}_{x}^{1}
$$

Comparing with $(8.27)_{3}$ yields:

$$
\begin{aligned}
& \mathcal{Y}_{y^{1}}^{1}=(2+\mathrm{s}) \mathcal{X}_{x}+\mathrm{r} \mathcal{X}+\mathrm{r} \mathcal{Y}^{1}+\mathrm{r} \mathcal{Y}^{2}+\mathrm{r} \mathcal{Y}_{x}^{1}, \\
& \mathcal{Y}_{y^{2}}^{2}=(3+\mathrm{s}) \mathcal{X}_{x}+\mathrm{r} \mathcal{X}+\mathrm{r} \mathcal{Y}^{1}+\mathrm{r} \mathcal{Y}^{2}+\mathrm{r} \mathcal{Y}_{x}^{1}
\end{aligned}
$$


These are $(8.18)_{5}$ and $(8.18)_{11}$. Differentiating these two equations with respect to $x$, replacing $\mathcal{Y}_{x}^{2}$ and erasing $\mathcal{Y}_{x x}^{1}$, we get:

$$
\begin{aligned}
& \mathcal{Y}_{x y^{1}}^{1}=(2+\mathrm{s}) \mathcal{X}_{x x}+\mathrm{r} \mathcal{X}+\mathrm{r} \mathcal{Y}^{1}+\mathrm{r} \mathcal{Y}^{2}+\mathrm{r} \mathcal{Y}_{x}^{1}+\mathrm{r} \mathcal{X}_{x}, \\
& \mathcal{Y}_{x y^{2}}^{2}=(3+\mathrm{s}) \mathcal{X}_{x x}+\mathrm{r} \mathcal{X}+\mathrm{r} \mathcal{Y}^{1}+\mathrm{r} \mathcal{Y}^{2}+\mathrm{r} \mathcal{Y}_{x}^{1}+\mathrm{r} \mathcal{X}_{x}
\end{aligned}
$$

We then replace this value of $\mathcal{Y}_{x y^{2}}^{2}$ in (8.29) and solve $\mathcal{X}_{x x}$ : this yields $(8.18)_{2}$.

To conclude, we replace $\mathcal{X}_{x x}$ so obtained in $(8.27)_{7}$ : this yields $(8.18)_{8}$. We replace $\mathcal{Y}_{y^{1}}^{1}$ and $\mathcal{Y}_{y^{2}}^{2}$ from $(8.32)$ in $(8.27)_{4}$ and in $(8.27)_{5}$ : this yields $(8.18)_{4}$ and this yields $(8.18)_{9}$. Thanks to $(8.18)_{2}$ (got) we observe that

$$
\mathrm{P}_{x}=\mathrm{P}+\mathrm{r} \mathcal{Y}_{x x}^{1}+\mathrm{r} \mathcal{Y}_{x}^{2}+\mathrm{r} \mathcal{X}_{x x}=\mathrm{P} .
$$

Differentiating $(8.18)_{4}$ (got) and $(8.18)_{9}$ (got) with respect to $x$ then yields $(8.18)_{7}$ and $(8.18)_{12}$. We replace $\mathcal{Y}_{y^{1}}^{1}$ and $\mathcal{Y}_{y^{2}}^{2}$ from $(8.18)_{5}$ (got) and $(8.18)_{11}$ (got) in $(8.27)_{2}$ : this yields $(8.18)_{6}$. Finally, to obtain the very last $(8.18)_{13}$, we differentiate $(8.18)_{10}$ (got) with respect to $x$.

The proof of Proposition 8.16 is complete.

We claim that the bound $\operatorname{dim} \mathfrak{S Y Y M}\left(\mathcal{E}_{4}\right) \leqslant 5$ is attained for the model (8.4). Indeed, with $0=\mathbf{r}=\mathrm{s}$ and $0=\mathbf{g}^{1}=\mathbf{g}^{2}=\mathbf{h}$ (whence $\mathbf{k}^{*}=0$ ) (8.24) is $\mathcal{Y}_{y^{2}}^{1}=0$ and then the seven equations (8.27) are:

$$
\left\{\begin{array}{l}
0=-\mathcal{Y}_{x}^{2}+2 x \mathcal{Y}_{x}^{1} \\
0=-\mathcal{Y}_{y^{1}}^{2}-2 x \mathcal{Y}_{y^{2}}^{2}+2 \mathcal{X}+2 \mathcal{Y}_{x}^{1}+2 x \mathcal{Y}_{y^{1}}^{1} \\
0=-\mathcal{Y}_{y^{2}}^{2}-\mathcal{X}_{x}+2 \mathcal{Y}_{y^{1}}^{1} \\
0=-\mathcal{X}_{y^{1}} \\
0=-\mathcal{X}_{y^{2}} \\
0=-\mathcal{Y}_{x x}^{1} \\
0=-2 \mathcal{Y}_{x y^{1}}^{1}+\mathcal{X}_{x x}
\end{array}\right.
$$

having the general solution

$$
\left\{\begin{array}{l}
\mathcal{X}=a-d+e x \\
\mathcal{Y}^{1}=b+d x+2 e y^{1} \\
\mathcal{Y}^{2}=c+2 a y^{1}+3 e y^{2}+d x x
\end{array}\right.
$$

depending on five parameters $a, b, c, d, e \in \mathbb{K}$. Five generators of $\mathfrak{S Y M M}\left(\mathcal{E}_{4}\right)$ are:

$$
\left\{\begin{aligned}
\mathcal{D} & :=x \partial_{x}+2 y^{1} \partial_{y^{1}}+3 y^{2} \partial_{y^{2}} \\
\mathcal{L}_{1} & :=-\partial_{x}+x \partial_{y^{1}}+x x \partial_{y^{2}} \\
\mathcal{L}_{1}^{\prime} & :=\partial_{x}+2 y^{1} \partial_{y^{2}} \\
\mathcal{L}_{2} & :=\partial_{y^{1}} \\
\mathcal{L}_{3} & :=\partial_{y^{2}}
\end{aligned}\right.
$$

The commutator table 


\begin{tabular}{l||l|l|l|l|l} 
& $\mathcal{D}$ & $\mathcal{L}_{1}$ & $\mathcal{L}_{1}^{\prime}$ & $\mathcal{L}_{2}$ & $\mathcal{L}_{3}$ \\
\hline \hline $\mathcal{D}$ & 0 & $-\mathcal{L}_{1}$ & $-\mathcal{L}_{1}^{\prime}$ & $-2 \mathcal{L}_{2}$ & $-3 \mathcal{L}_{3}$ \\
\hline $\mathcal{L}_{1}$ & $\mathcal{L}_{1}$ & 0 & $-\mathcal{L}_{2}$ & 0 & 0 \\
\hline $\mathcal{L}_{1}^{\prime}$ & $\mathcal{L}_{1}^{\prime}$ & $\mathcal{L}_{2}$ & 0 & $-2 \mathcal{L}_{3}$ & 0 \\
\hline $\mathcal{L}_{2}$ & $2 \mathcal{L}_{2}$ & 0 & $2 \mathcal{L}_{3}$ & 0 & 0 \\
\hline $\mathcal{L}_{3}$ & $3 \mathcal{L}_{3}$ & 0 & 0 & 0 & 0
\end{tabular}

Table 3.

shows that the subalgebra spanned by $\mathcal{L}_{1}, \mathcal{L}_{1}^{\prime}, \mathcal{L}_{2}, \mathcal{L}_{3}$ is isomorphic to the unique irreducible 4-dimensional nilpotent Lie algebra $\mathfrak{n}_{4}^{1}$ ([OV1994, BES2005]). Then $\mathfrak{S Y M M ( \mathcal { E } _ { 4 } )}$ is a semidirect product of $\mathbb{K}$ with $\mathfrak{n}_{4}^{1}$. The author ignores whether it is rigid. The following accessible research will be pursued in a subsequent publication.

Open problem 8.38. Classify systems $\left(\mathcal{E}_{4}\right)$ up to point transformations. Deduce a complete classification, up to local biholomorphisms, of all real analytic generic submanifolds of codimension 2 in $\mathbb{C}^{3}$, valid at a Zariski-generic point.

8.39. Almost everywhere rigid hypersurfaces. When studying and classifying differential objects, it is essentially no restriction to assume their Lie symmetry groups to be of dimension $\geqslant 1$, the study of objects having no infinitesimal symmetries being an independent field of research. In particular, if $M \subset \mathbb{C}^{n+1}(n \geqslant 1)$ is a connected real analytic hypersurface, we may suppose that $\operatorname{dim} \mathfrak{h o l}(M) \geqslant 1$, at least. So let $\mathcal{L}$ be a nonzero holomorphic vector field with $\mathcal{L}+\overline{\mathcal{L}}$ tangent to $M$.

Lemma 8.40. ([Ca1932a, St1996, BER1999]) If in addition $M$ is finitely nondegenerate, then

$$
\Sigma:=\left\{p \in M: \mathcal{L}(p) \in T_{p}^{c} M\right\}
$$

is a proper real analytic subset of $M$.

In other words, at every point $p$ belonging to the Zariski-dense subset $M \backslash \Sigma$, the real nonzero vector $\mathcal{L}(p)+\overline{\mathcal{L}(p)} \in T_{p} M$ supplements $T_{p}^{c} M$. Straightening $\mathcal{L}$ in a neighborhood of $p$, there exist local coordinates $t=\left(z_{1}, \ldots, z_{n}, w\right)$ with $T_{0}^{c} M=\{w=0\}$, $T_{0} M=\{\operatorname{Im} w=0\}$, whence $M$ is given by $\operatorname{Im} w=h(z, \bar{z}, \operatorname{Re} w)$, and with $\mathcal{L}=\frac{\partial}{\partial w}$. The tangency of $\frac{\partial}{\partial w}+\frac{\partial}{\partial \bar{w}}=\frac{\partial}{\partial u}$ to $M$ entails that $h$ is indendepent of $u$. Then the complex equation of $M$ is of the precise form

$$
w=\bar{w}+i \bar{\Theta}(z, \bar{z}),
$$

with $\bar{\Theta}=2 h$ simply. The reality of $h$ reads $\bar{\Theta}(z, \bar{z}) \equiv \Theta(\bar{z}, z)$.

Definition 8.43. A real analytic hypersurface $M \subset \mathbb{C}^{n+1}$ is called rigid at one of its points $p$ if there exists $\mathcal{L} \in \mathfrak{h o l}(M)$ with

$$
T_{p} M=T_{p}^{c} M \oplus \mathbb{R}(\mathcal{L}(p)+\overline{\mathcal{L}}(p)) .
$$

Similar elementary facts hold for general submanifolds of solutions.

Lemma 8.45. With $n \geqslant 1$ and $m=1$, let $\mathcal{M}$ be a (connected) submanifold of solutions that is solvable with respect to the parameters. If there exists a nonzero $\mathcal{L}+\mathcal{L}^{*} \in \mathfrak{S} \mathfrak{Y M}(\mathcal{M})$, then at Zariski-generic points $p \in \mathcal{M}$, we have $\mathcal{L}(p) \notin \mathrm{F}_{\mathrm{v}}(p)$ and there exist local coordinates centered at $p$ in which $\mathcal{L}=\frac{\partial}{\partial y}, \mathcal{L}^{*}=\frac{\partial}{\partial b}$, whence $\mathcal{M}$ has equation of the form

$$
y=b+\Pi(x, a),
$$

with $\Pi$ independent of $b$. 
The associated system $\left(\mathcal{E}_{\mathcal{M}}\right)$ has then equations $F_{\alpha}$ that are all independent of $y$.

8.47. Study of the Lie symmetries of $\left(\mathcal{E}_{5}\right)$. In Example 1.28 , it is thus essentially no restriction to assume the hypersurface $M \subset \mathbb{C}^{3}$ to be rigid.

Theorem 8.48. ([GM2003b, FK2005a, FK2005b]) The model hypersurface $M_{0}$ of equation

$$
w=\bar{w}+i \frac{2 z^{1} \bar{z}^{1}+z^{1} z^{1} \bar{z}^{2}+\bar{z}^{1} \bar{z}^{1} z^{2}}{1-z^{2} \bar{z}^{2}}
$$

has transitive Lie symmetry algebra $\mathfrak{h o l}\left(M_{0}\right)$ isomorphic to $\mathfrak{s o}(3,2)$ and is locally biholomorphic to a neighborhood of every geometrically smooth point of the tube

$$
\left(\operatorname{Re} w^{\prime}\right)^{2}=\left(\operatorname{Re} z_{1}^{\prime}\right)^{2}+\left(\operatorname{Re} z_{1}^{\prime}\right)^{3}
$$

over the standard cone of $\mathbb{R}^{3}$. Both are Levi-degenerate with Levi form of rank 1 at every point and are 2-nondegenerate. The associated PDE system $\left(\mathcal{E}_{\mathcal{M}_{0}}\right)$

$$
y_{x^{2}}=\frac{1}{4}\left(y_{x^{1}}\right)^{2}, \quad y_{x^{1} x^{1} x^{1}}=0
$$

(plus other equations obtained by cross differentiation) has infinitesimal Lie symmetry algebra isomorphic to $\mathfrak{s o}(5, \mathbb{C})$, the complexification $\mathfrak{s o}(3,2) \otimes \mathbb{C}$.

Through tentative issues ([Eb2006, GM2006]), it has been suspected that $M_{0}$ is the right model in the category of real analytic hypersurfaces $M \subset \mathbb{C}^{3}$ having Levi form of rank 1 that are 2-nondegenerate everywhere. Based on the rigidity of the simple Lie algebra $\mathfrak{s o}(5, \mathbb{C})$ (Theorem 5.15), Theorem 8.105 below will confirm this expectation.

8.52. Preparation. Thus, translating the considerations to the PDE language, with $n=2$ and $m=1$, consider a submanifold of solutions of the form

$$
\begin{aligned}
y & =b+\Pi(x, a) \\
& =b+\frac{2 x^{1} a^{1}+x^{1} x^{1} a^{2}+a^{1} a^{1} x^{2}}{1-x^{2} a^{2}}+\mathrm{O}_{4},
\end{aligned}
$$

where $\mathrm{O}_{4}$ is a function of $(x, a)$ only. The term $2 x^{1} a^{1}$ corresponds to a Levi form of rank $\geqslant 1$ at every point. The term $x^{1} x^{1} a^{2}$ guarantees solvability with respect to the parameters (compare Definition 2.12). Let us develope

$$
\Pi(x, a)=\sum_{k_{1}, k_{2} \geqslant 0} \sum_{l_{1}, l_{2} \geqslant 0} \Pi_{k_{1}, k_{2}, l_{1}, l_{2}}\left(x^{1}\right)^{k_{1}}\left(x^{2}\right)^{k_{2}}\left(a^{1}\right)^{l_{1}}\left(a^{2}\right)^{l_{2}},
$$

with $\prod_{k_{1}, k_{2}, l_{1}, l_{2}} \in \mathbb{K}$. Of course, $\Pi_{1,0,1,0}=2, \Pi_{2,0,0,1}=1$ and $\Pi_{0,1,2,0}=1$.

Lemma 8.55. A transformation belonging to $\mathrm{G}_{\mathrm{v}, \mathrm{p}}$ insures

$$
\begin{array}{llll}
\Pi_{k_{1}, k_{2}, 0,0}=0, & k_{1}+k_{2} \geqslant 0, & \Pi_{0,0, l_{1}, l_{2}}=0, & l_{1}+l_{2} \geqslant 0, \\
\Pi_{k_{1}, k_{2}, 1,0}=0, & k_{1}+k_{2} \geqslant 2, & \Pi_{1,0, l_{1}, l_{2}}=0, & l_{1}+l_{2} \geqslant 2, \\
\Pi_{k_{1}, k_{2}, 2,0}=0, & k_{1}+k_{2} \geqslant 2, & \Pi_{2,0, l_{1}, l_{2}}=0, & l_{1}+l_{2} \geqslant 2 .
\end{array}
$$

Proof. Lemma 7.11 achieves the first line. The monomial $x^{1}$ being factored by $\left[a^{1}+\right.$ $\left.\mathrm{O}_{2}(a)\right]$, we set $a^{1}:=a^{1}+\mathrm{O}_{2}(a)$ to achieve $\Pi_{1,0, l_{1}, l_{2}}=0, l_{1}+l_{2} \geqslant 2$. As in the proof of Lemma 7.18, we pass to the dual equation $b=y-\Pi(x, a)$ to complete $\Pi_{k_{1}, k_{2}, 1,0}=0$, $k_{1}+k_{2} \geqslant 2$. Finally, $x^{1} x^{1}$ is factored by $\left[a^{2}+\mathrm{O}_{2}(a)\right]$, so we proceed similarly to achieve the third line. 
Since $\Pi(x, a)$ is assumed to be independent of $b$, the assumption that the Levi form of $M \subset \mathbb{C}^{3}$ has exactly rank 1 at every point translates to:

$$
0 \equiv\left|\begin{array}{ll}
\Pi_{x^{1} a^{1}} & \Pi_{x^{1} a^{2}} \\
\prod_{x^{2} a^{1}} & \Pi_{x^{2} a^{2}}
\end{array}\right|
$$

For later use, it is convenient to develope somehow $\Pi$ with respect to the powers of $\left(a^{1}, a^{2}\right)$ :

$$
\begin{aligned}
y=b+ & \frac{2 x^{1} a^{1}+x^{1} x^{1} a^{2}+a^{1} a^{1} x^{2}}{1-x^{2} a^{2}}+ \\
& +a^{2} \mathbf{b}(x)+a^{1} a^{2} \mathbf{d}(x)+a^{2} a^{2} \mathbf{e}(x)+a^{1} a^{1} a^{1} \mathbf{f}(x)+a^{1} a^{1} a^{2} \mathbf{g}(x)+ \\
& +\left(a^{1}\right)^{4} \mathrm{R}+\left(a^{1}\right)^{3} a^{2} \mathrm{R}+a^{1}\left(a^{2}\right)^{2} \mathrm{R}+\left(a^{2}\right)^{3} \mathrm{R}
\end{aligned}
$$

with $\mathrm{R}=\mathrm{R}(x, a)$ being an unspecified remainder. Thanks to the previous lemma, the coefficients a of $a^{1}$ and $\mathbf{c}$ of $a^{1} a^{1}$ must vanish. The function $\mathbf{b}$ is an $\mathrm{O}_{3}$.

Lemma 8.59. The function $\mathbf{b}$ depends only on $x^{1}$, is an $\mathrm{O}_{3}\left(x^{1}\right)$ and the function $\mathbf{g}$ satisfies $\mathbf{g}_{x^{2} x^{2}}(0)=0$.

Proof. Developing $\left[1-x^{2} a^{2}\right]^{-1}=1+x^{2} a^{2}+\left(x^{2} a^{2}\right)^{2}+\mathrm{O}_{3}\left(x^{2} a^{2}\right)$, inserting the right hand side of

$$
\begin{aligned}
y-b= & a^{1}\left[2 x^{1}\right]+a^{2}\left[x^{1} x^{1}+\mathbf{b}(x)\right]+a^{1} a^{1}\left[x^{2}\right]+a^{1} a^{2}\left[2 x^{1} x^{2}+\mathbf{d}(x)\right]+ \\
& +a^{2} a^{2}\left[x^{1} x^{1} x^{2}+\mathbf{e}(x)\right]+a^{1} a^{1} a^{1}[\mathbf{f}(x)]+a^{1} a^{1} a^{2}\left[x^{2} x^{2}+\mathbf{g}(x)\right]+ \\
& +\left(a^{1}\right)^{4} \mathrm{R}+\left(a^{1}\right)^{3} a^{2} \mathrm{R}+a^{1}\left(a^{2}\right)^{2} \mathrm{R}+\left(a^{2}\right)^{3} \mathrm{R}
\end{aligned}
$$

in the determinant (8.57) and selecting the coefficients of cst., of $a^{1}$, of $a^{2}$ and of $a^{1} a^{1}$, we get four PDEs:

$$
\begin{aligned}
& 0=2 \mathbf{b}_{x^{2}} \\
& 0=2 \mathbf{d}_{x^{2}}-2 \mathbf{b}_{x^{1}} \\
& 0=4 \mathbf{e}_{x^{2}}-2 x^{1} \mathbf{d}_{x^{2}}-2 x^{1} \mathbf{b}_{x^{1}}-\mathbf{d}_{x^{2}} \mathbf{b}_{x^{1}} \\
& 0=2 \mathbf{g}_{x^{2}}-2 \mathbf{d}_{x^{1}}-\left[6 x^{1}+3 \mathbf{b}_{x^{1}}\right] \mathbf{f}_{x^{2}} .
\end{aligned}
$$

The first one yields $\mathbf{b}=\mathbf{b}\left(x^{1}\right)$, which must be an $\mathrm{O}_{3}\left(x^{1}\right)$, because the whole remainder is an $\mathrm{O}_{4}$. Differentiating the fourth with respect to $x^{2}$, it then follows that $\mathbf{g}_{x^{2} x^{2}}(0)=0$.

8.62. Associated PDE system $\left(\mathcal{E}_{5}\right)$. Next, differentiating (8.60) with respect to $x^{1}$, to $x^{1} x^{1}$ and to $x^{1} x^{1} x^{1}$, we compute $y_{x^{1}}$ and $y_{x^{1} x^{1}}$, we substitute $y_{1}$ and $y_{1,1}$ and we push the monomials $a^{2} a^{2}, a^{1} a^{1} a^{1}$ and $a^{1} a^{1} a^{2}$ in the remainder:

$$
\begin{aligned}
y_{1} & =2 a^{1}+a^{2}\left[2 x^{1}+\mathbf{b}_{x^{1}}\right]+a^{1} a^{2}\left[2 x^{2}+\mathbf{d}_{x^{1}}\right]+\left(a^{2}\right)^{2} \mathrm{R}+\left(a^{1}\right)^{3} \mathrm{R}+\left(a^{1}\right)^{2} a^{2} \mathrm{R}, \\
y_{1,1} & =a^{2}\left[2+\mathbf{b}_{x^{1} x^{1}}\right]+a^{1} a^{2}\left[\mathbf{d}_{x^{1} x^{1}}\right]+\left(a^{2}\right)^{2} \mathrm{R}+\left(a^{1}\right)^{3} \mathrm{R}+\left(a^{1}\right)^{2} a^{2} \mathrm{R}, \\
y_{1,1,1} & =a^{2}\left[\mathbf{b}_{x^{1} x^{1} x^{1}}\right]+a^{1} a^{2}\left[\mathbf{d}_{x^{1} x^{1} x^{1}}\right]+\left(a^{2}\right)^{2} \mathrm{R}+\left(a^{1}\right)^{3} \mathrm{R}+\left(a^{1}\right)^{2} a^{2} \mathrm{R} .
\end{aligned}
$$

Here, the written remainder cannot incorporate $a^{1} a^{1}$, so it is said that the coefficient of $a^{1} a^{1}$ does vanish in each equation above. Solving for $a^{1}$ and $a^{2}$ from the first two 
equations, we get

(8.64)

$$
\left\{\begin{aligned}
a^{1}= & \frac{1}{2} y_{1}-y_{1,1}\left[\frac{2 x^{1}+\mathbf{b}_{x^{1}}}{4+2 \mathbf{b}_{x^{1} x^{1}}}\right]-y_{1} y_{1,1}\left[\frac{2 x^{2}+\mathbf{d}_{x^{1}}}{8+4 \mathbf{b}_{x^{1} x^{1}}}\right]+ \\
& +\left(y_{1,1}\right)^{2} \mathrm{R}+\left(y_{1}\right)^{3} \mathrm{R}+\left(y_{1}\right)^{2} y_{1,1} \mathbf{R} \\
a^{2}= & y_{1,1}\left[\frac{1}{2+\mathbf{b}_{x^{1} x^{1}}}\right]-y_{1} y_{1,1}\left[\frac{\mathbf{d}_{x^{1} x^{1}}}{2\left(2+\mathbf{b}_{x^{1} x^{1}}\right)^{2}}\right]+\left(y_{1,1}\right)^{2} \mathrm{R}+\left(y_{1}\right)^{3} \mathrm{R}+\left(y_{1}\right)^{2} y_{1,1} \mathbf{R} .
\end{aligned}\right.
$$

We then get (notice the change of remainder):

(8.65)

$$
\begin{aligned}
a^{1} a^{1} & =\frac{1}{4}\left(y_{1}\right)^{2}-y_{1} y_{1,1}\left[\frac{2 x^{1}+\mathbf{b}_{x^{1}}}{4+2 \mathbf{b}_{x^{1} x^{1}}}\right]-\left(y_{1}\right)^{2} y_{1,1}\left[\frac{2 x^{2}+\mathbf{d}_{x^{1}}}{8+4 \mathbf{b}_{x^{1} x^{1}}}\right]+\left(y_{1}\right)^{3} \mathrm{R}+\left(y_{1,1}\right)^{2} \mathrm{R}, \\
a^{1} a^{2} & =y_{1} y_{1,1}\left[\frac{1}{4+2 \mathbf{b}_{x^{1} x^{1}}}\right]-\left(y_{1}\right)^{2} y_{1,1}\left[\frac{\mathbf{d}_{x^{1} x^{1}}}{\left(4+2 \mathbf{b}_{x^{1} x^{1}}\right)^{2}}\right]+\left(y_{1}\right)^{3} \mathrm{R}+\left(y_{1,1}\right)^{2} \mathrm{R}, \\
a^{2} a^{2} & =\left(y_{1}\right)^{3} \mathrm{R}+\left(y_{1,1}\right)^{2} \mathrm{R}, \\
a^{1} a^{1} a^{1} & =-\left(y_{1}\right)^{2} y_{1,1}\left[\frac{6 x^{1}+3 \mathbf{b}_{x^{1}}}{16+8 \mathbf{b}_{x^{1} x^{1}}}\right]\left(y_{1}\right)^{3} \mathrm{R}+\left(y_{1,1}\right)^{2} \mathrm{R}, \\
a^{1} a^{1} a^{2} & =\left(y_{1}\right)^{2} y_{1,1}\left[\frac{1}{8+4 \mathbf{b}_{x^{1} x^{1}}}\right]+\left(y_{1}\right)^{3} \mathrm{R}+\left(y_{1,1}\right)^{2} \mathrm{R} .
\end{aligned}
$$

Differentiating (8.60) with respect to $x^{2}$, substituting $y_{2}$ for $y_{x^{2}}$ and replacing $\mathbf{d}_{x^{2}}$ by $\mathbf{b}_{x^{1}}$ thanks to $(8.61)_{2}$, we get

(8.66)

$$
\begin{aligned}
y_{2}= & a^{1} a^{1}+a^{1} a^{2}\left[2 x^{1}+\mathbf{b}_{x^{1}}\right]+a^{2} a^{2}\left[x^{1} x^{1}+\mathbf{e}_{x^{2}}\right]+a^{1} a^{1} a^{1}\left[\mathbf{f}_{x^{2}}\right]+a^{1} a^{1} a^{2}\left[2 x^{2}+\mathbf{g}_{x^{2}}\right]+ \\
& +\left(a^{1}\right)^{4} \mathrm{R}+\left(a^{1}\right)^{3} a^{2} \mathrm{R}+a^{1}\left(a^{2}\right)^{2} \mathrm{R}+\left(a^{2}\right)^{3} \mathrm{R} .
\end{aligned}
$$

Replacing the monomials (8.65), we finally obtain:

$$
\begin{aligned}
y_{2}=\frac{1}{4}\left(y_{1}\right)^{2}+ & \left(y_{1}\right)^{2} y_{1,1}\left[\frac{2 \mathbf{g}_{x^{2}}-2 \mathbf{d}_{x^{1}}-\left(6 x^{1}+3 \mathbf{b}_{x^{1}}\right) \mathbf{f}_{x^{2}}}{16+8 \mathbf{b}_{x^{1} x^{1}}}-\frac{\left(2 x^{1}+\mathbf{b}_{x^{1}}\right) \mathbf{d}_{x^{1} x^{1}}}{\left(4+2 \mathbf{b}_{x^{1} x^{1}}\right)^{2}}\right]+ \\
& +\left(y_{1}\right)^{3} \mathrm{R}+\left(y_{1,1}\right)^{2} \mathrm{R} .
\end{aligned}
$$

Thanks to $(8.61)_{4}$, the first (big) coefficient of $\left(y_{1}\right)^{2} y_{1,1}$ is zero; then the remainder coefficient is an $\mathrm{O}\left(x^{1}\right)$, hence vanishes at $x=0$, together with its partial first derivative with respect to $x^{2}$. Accordingly, by $\mathrm{s}^{*}=\mathrm{s}^{*}\left(x^{1}, x^{2}\right)$, we will denote an unspecified function satisfying

$$
\mathrm{s}^{*}(0)=0 \quad \text { and } \quad \mathrm{s}_{x^{2}}^{*}(0)=0 \text {. }
$$

Lemma 8.69. The skeleton of the PDE system $\left(\mathcal{E}_{5}\right)$ associated to the submanifold (8.58) possesses three main equations of the form

$$
\left(\Delta_{\mathcal{E}_{5}}\right)\left\{\begin{aligned}
y_{2}= & \frac{1}{4}\left(y_{1}\right)^{2}+ \\
& \left(y_{1}\right)^{3} \mathbf{r}+\left(y_{1}\right)^{4} \mathbf{r}+\left(y_{1}\right)^{5} \mathbf{r}+\left(y_{1}\right)^{6} \mathbf{R}+ \\
& +y_{1,1}\left[\left(y_{1}\right)^{2} \mathbf{s}^{*}+\left(y_{1}\right)^{3} \mathbf{r}+\left(y_{1}\right)^{4} \mathbf{r}+\left(y_{1}\right)^{5} \mathbf{r}\right]+\left(y_{1,1}\right)^{2} \mathbf{R}, \\
y_{1,2}=\frac{1}{2} y_{1} y_{1,1} & +\left(y_{1}\right)^{3} \mathbf{r}+\left(y_{1}\right)^{4} \mathbf{r}+\left(y_{1}\right)^{5} \mathbf{r}+\left(y_{1}\right)^{6} \mathbf{R}+ \\
& +y_{1,1}\left[\left(y_{1}\right)^{2} \mathbf{r}+\left(y_{1}\right)^{3} \mathbf{r}+\left(y_{1}\right)^{4} \mathbf{r}+\left(y_{1}\right)^{5} \mathbf{r}\right]+\left(y_{1}\right)^{6} \mathbf{R}, \\
y_{1,1,1}=\left(y_{1}\right)^{3} \mathbf{r}+ & \left(y_{1}\right)^{4} \mathbf{r}+y_{1,1}\left[\mathbf{r}+y_{1} \mathbf{r}+\left(y_{1}\right)^{2} \mathbf{r}+\left(y_{1}\right)^{3} \mathbf{r}\right]+ \\
& +\left(y_{1,1}\right)^{2}\left[\mathbf{r}+y_{1} \mathbf{r}+\left(y_{1}\right)^{2} \mathbf{r}+\left(y_{1}\right)^{3} \mathbf{r}\right]+\left(y_{1,1}\right)^{3} \mathbf{R},
\end{aligned}\right.
$$


where the letter $\mathrm{r}$ denotes an unspecified function of $\left(x^{1}, x^{2}\right)$, and where the coefficient $\mathrm{s}^{*}$ of $\left(y_{1}\right)^{2} y_{1,1}$ in the first equation satisfies (8.68).

Proof. To get the second equation, we compute:

$$
\begin{aligned}
y_{1,2} & =a^{1} a^{2}\left[2+\mathbf{b}_{x^{1} x^{1}}\right]+a^{1} a^{1} a^{2}\left[\mathbf{g}_{x^{1} x^{2}}\right]+\left(a^{1}\right)^{3} \mathrm{R}+\left(a^{2}\right)^{2} \mathrm{R} \\
& =\frac{1}{2} y_{1} y_{1,1}+\left(y_{1}\right)^{2} y_{1,1} \mathbf{r}+\left(y_{1}\right)^{3} \mathrm{R}+\left(y_{1,1}\right)^{2} \mathrm{R} .
\end{aligned}
$$

The third equation is got similarly from $(8.63)_{3}$. To conclude, we develope the first two equations $\bmod \left[\left(y_{1}\right)^{6},\left(y_{1,1}\right)^{2}\right]$ and the third one $\bmod \left[\left(y_{1}\right)^{4},\left(y_{1,1}\right)^{3}\right]$.

This precise skeleton will be referred to as $\Delta_{\mathcal{E}_{5}}$ in the sequel. With the letter $r$, the computation rules are cst. $r=r+r=r+s^{*}=r \cdot r=r$; sometimes, $s^{*}$ may be replaced plainly by $r$.

8.71. Infinitesimal Lie symmetries of $\left(\mathcal{E}_{5}\right)$. Letting $\mathcal{L}=\mathcal{X}^{1} \frac{\partial}{\partial x^{1}}+\mathcal{X}^{2} \frac{\partial}{\partial x^{2}}+\mathcal{Y} \frac{\partial}{\partial y}$ be a candidate infinitesimal Lie symmetry and applying

$$
\begin{aligned}
\mathcal{L}^{(3)}= & \mathcal{X}^{1} \frac{\partial}{\partial x^{1}}+\mathcal{X}^{2} \frac{\partial}{\partial x^{2}}+\mathcal{Y} \frac{\partial}{\partial y}+\mathbf{Y}_{1} \frac{\partial}{\partial y_{1}}+\mathbf{Y}_{2} \frac{\partial}{\partial y_{2}}+ \\
& +\mathbf{Y}_{1,1} \frac{\partial}{\partial y_{1,1}}+\mathbf{Y}_{1,2} \frac{\partial}{\partial y_{1,2}}+\mathbf{Y}_{2,1} \frac{\partial}{\partial y_{2,1}}+\mathbf{Y}_{2,2} \frac{\partial}{\partial y_{2,2}}+ \\
& +\mathbf{Y}_{1,1,1} \frac{\partial}{\partial y_{1,1,1}}+\cdots+\mathbf{Y}_{2,2,2} \frac{\partial}{\partial y_{2,2,2}}
\end{aligned}
$$

to the skeleton $\Delta_{\mathcal{E}_{5}}$, we obtain firstly, computing $\bmod \left[\left(y_{1}\right)^{5}, y_{1,1}\right]$ :

$$
\begin{aligned}
0 \equiv & -\mathbf{Y}_{2}+\frac{1}{2} y_{1} \mathbf{Y}_{1}+ \\
& +\left(y_{1}\right)^{3} \mathbf{r} \mathcal{X}^{1}+\left(y_{1}\right)^{4} \mathbf{r} \mathcal{X}^{1}+\left(y_{1}\right)^{3} \mathbf{r} \mathcal{X}^{2}+\left(y_{1}\right)^{4} \mathbf{r} \mathcal{X}^{2}+ \\
& +\mathbf{Y}_{1}\left[\left(y_{1}\right)^{2} \mathbf{r}+\left(y_{1}\right)^{3} \mathbf{r}+\left(y_{1}\right)^{4} \mathbf{r}\right]+ \\
& +\mathbf{Y}_{1,1}\left[\left(y_{1}\right)^{2} \mathbf{s}^{*}+\left(y_{1}\right)^{3} \mathbf{r}+\left(y_{1}\right)^{4} \mathbf{r}\right]
\end{aligned}
$$

secondly, computing $\bmod \left[\left(y_{1}\right)^{5}, y_{1,1}\right]$ :

$$
\begin{aligned}
0 \equiv & -\mathbf{Y}_{1,2}+\frac{1}{2} y_{1} \mathbf{Y}_{1,1}+ \\
& +\left(y_{1}\right)^{3} \mathbf{r} \mathcal{X}^{1}+\left(y_{1}\right)^{4} \mathbf{r} \mathcal{X}^{1}+\left(y_{1}\right)^{3} \mathbf{r} \mathcal{X}^{2}+\left(y_{1}\right)^{4} \mathbf{r} \mathcal{X}^{2}+ \\
& +\mathbf{Y}_{1}\left[\left(y_{1}\right)^{2} \mathbf{r}+\left(y_{1}\right)^{3} \mathbf{r}+\left(y_{1}\right)^{4} \mathbf{r}\right]+ \\
& +\mathbf{Y}_{1,1}\left[\left(y_{1}\right)^{2} \mathbf{r}+\left(y_{1}\right)^{3} \mathbf{r}+\left(y_{1}\right)^{4} \mathbf{r}\right],
\end{aligned}
$$

and thirdly, computing $\bmod \left[\left(y_{1}\right)^{3},\left(y_{1,1}\right)^{2}\right]$ :

$$
\begin{aligned}
0 \equiv & -\mathbf{Y}_{1,1,1}+y_{1,1} \mathcal{X}^{1}+y_{1,1} \mathcal{X}^{2}+ \\
& +y_{1,1} y_{1} \mathcal{X}^{1}+y_{1,1} y_{1} \mathcal{X}^{2}+y_{1,1}\left(y_{1}\right)^{2} \mathcal{X}^{1}+y_{1,1}\left(y_{1}\right)^{2} \mathcal{X}^{2}+ \\
& +\mathbf{Y}_{1}\left[\left(y_{1}\right)^{2} \mathbf{r}\right]+\mathbf{Y}_{1,1}\left[\mathbf{r}+y_{1} \mathbf{r}+\left(y_{1}\right)^{2} \mathbf{r}\right]+y_{1,1} \mathbf{Y}_{1}\left[\mathbf{r}+y_{1} \mathbf{r}+\left(y_{1}\right)^{2} \mathbf{r}\right]+ \\
& \quad+y_{1,1} \mathbf{Y}_{1,1}\left[\mathbf{r}+y_{1} \mathbf{r}+\left(y_{1}\right)^{2} \mathbf{r}\right] .
\end{aligned}
$$

Specializing to $n=2$ the formulas (3.9)(II), (3.20)(II) and (3.24)(II), we get $\mathbf{Y}_{1}, \mathbf{Y}_{2}$, $\mathbf{Y}_{1,1}, \mathbf{Y}_{1,2}$ and $\mathbf{Y}_{1,1,1}$ :

$$
\begin{aligned}
& \mathbf{Y}_{1}=\mathcal{Y}_{x^{1}}+\left[\mathcal{Y}_{y}-\mathcal{X}_{x^{1}}^{1}\right] y_{1}+\left[-\mathcal{X}_{x^{1}}^{2}\right] y_{2}+\left[-\mathcal{X}_{y}^{1}\right]\left(y_{1}\right)^{2}+\left[-\mathcal{X}_{y}^{2}\right] y_{1} y_{2} \\
& \mathbf{Y}_{2}=\mathcal{Y}_{x^{2}}+\left[-\mathcal{X}_{x^{2}}^{1}\right] y_{1}+\left[\mathcal{Y}_{y}-\mathcal{X}_{x^{2}}^{2}\right] y_{2}+\left[-\mathcal{X}_{y}^{1}\right] y_{1} y_{2}+\left[-\mathcal{X}_{y}^{2}\right] y_{2} y_{2}
\end{aligned}
$$


(8.78)

$$
\left\{\begin{aligned}
\mathbf{Y}_{1,1}= & \mathcal{Y}_{x^{1} x^{1}}+\left[2 \mathcal{Y}_{x^{1} y}-\mathcal{X}_{x^{1} x^{1}}^{1}\right] y_{1}+\left[-\mathcal{X}_{x^{1} x^{1}}^{2}\right] y_{2}+\left[\mathcal{Y}_{y y}-2 \mathcal{X}_{x^{1} y}^{1}\right]\left(y_{1}\right)^{2}+ \\
& +\left[-2 \mathcal{X}_{x^{1} y}^{2}\right] y_{1} y_{2}+\left[-\mathcal{X}_{y y}^{1}\right]\left(y_{1}\right)^{3}+\left[-\mathcal{X}_{y y}^{2}\right]\left(y_{1}\right)^{2} y_{2}+ \\
& +\left[\mathcal{Y}_{y}-2 \mathcal{X}_{x^{1}}^{1}\right] y_{1,1}+\left[-2 \mathcal{X}_{x^{1}}^{2}\right] y_{1,2}+\left[-3 \mathcal{X}_{y}^{1}\right] y_{1} y_{1,1}+ \\
& +\left[-\mathcal{X}_{y}^{2}\right] y_{2} y_{1,1}+\left[-2 \mathcal{X}_{y}^{2}\right] y_{1} y_{1,2} .
\end{aligned}\right.
$$

$$
\left\{\begin{aligned}
\mathbf{Y}_{1,2}= & \mathcal{Y}_{x^{1} x^{2}}+\left[\mathcal{Y}_{x^{2} y}-\mathcal{X}_{x^{1} x^{2}}^{1}\right] y_{1}+\left[\mathcal{Y}_{x^{1} y}-\mathcal{X}_{x^{1} x^{2}}^{2}\right] y_{2}+ \\
& +\left[-\mathcal{X}_{x^{2} y}^{1}\right]\left(y_{1}\right)^{2}+\left[\mathcal{Y}_{y y}-\mathcal{X}_{x^{2} y}^{2}-\mathcal{X}_{x^{1} y}^{1}\right] y_{1} y_{2}+\left[-\mathcal{X}_{x^{1} y}^{2}\right] y_{2} y_{2}+ \\
& +\left[-\mathcal{X}_{y y}^{1}\right]\left(y_{1}\right)^{2} y_{2}+\left[-\mathcal{X}_{y y}^{2}\right] y_{1}\left(y_{2}\right)^{2}+ \\
& +\left[-\mathcal{X}_{x^{2}}^{1}\right] y_{1,1}+\left[\mathcal{Y}_{y}-\mathcal{X}_{x^{2}}^{2}-\mathcal{X}_{x^{1}}^{1}\right] y_{1,2}+\left[-\mathcal{X}_{x^{1}}^{2}\right] y_{2,2}+ \\
& +\left[-2 \mathcal{X}_{y}^{1}\right] y_{1} y_{1,2}+\left[-2 \mathcal{X}_{y}^{2}\right] y_{2} y_{1,2} .
\end{aligned}\right.
$$

$$
\left\{\begin{aligned}
\mathbf{Y}_{1,1,1}= & \mathcal{Y}_{x^{1} x^{1} x^{1}}+\left[3 \mathcal{Y}_{x^{1} x^{1} y}-\mathcal{X}_{x^{1} x^{1} x^{1}}^{1}\right] y_{1}+\left[-\mathcal{X}_{x^{1} x^{1} x^{1}}^{2}\right] y_{2}+ \\
& +\left[3 \mathcal{Y}_{x^{1} y y}-3 \mathcal{X}_{x^{1} x^{1} y}^{1}\right]\left(y_{1}\right)^{2}+\left[-3 \mathcal{X}_{x^{1} x^{1} y}^{2}\right] y_{1} y_{2}+ \\
& +\left[\mathcal{Y}_{y y y}-3 \mathcal{X}_{x^{1} y y}^{1}\right]\left(y_{1}\right)^{3}+\left[-3 \mathcal{X}_{x^{1} y y}^{2}\right]\left(y_{1}\right)^{2} y_{2}+ \\
& +\left[-\mathcal{X}_{y y y}^{1}\right]\left(y_{1}\right)^{4}+\left[-\mathcal{X}_{y y y}^{2}\right]\left(y_{1}\right)^{3} y_{2}+ \\
& +\left[3 \mathcal{Y}_{x^{1} y}-3 \mathcal{X}_{x^{1} x^{1}}^{1}\right] y_{1,1}+\left[-3 \mathcal{X}_{x^{1} x^{1}}^{2}\right] y_{1,2}+ \\
& +\left[3 \mathcal{Y}_{y y}-9 \mathcal{X}_{x^{1} y}^{1}\right] y_{1} y_{1,1}+\left[-3 \mathcal{X}_{x^{1} y}^{2}\right] y_{2} y_{1,1}+ \\
& +\left[-6 \mathcal{X}_{x^{1} y}^{2}\right] y_{1} y_{1,2}+\left[-6 \mathcal{X}_{y y}^{1}\right]\left(y_{1}\right)^{2} y_{1,1}+\left[-3 \mathcal{X}_{y y}^{2}\right] y_{1} y_{2} y_{1,1}+ \\
& +\left[-3 \mathcal{X}_{y y}^{2}\right]\left(y_{1}\right)^{2} y_{1,2}+\left[-3 \mathcal{X}_{y}^{1}\right]\left(y_{1,1}\right)^{2}+\left[-3 \mathcal{X}_{y}^{2}\right] y_{1,1} y_{1,2}+ \\
& +\left[\mathcal{Y}_{y}-3 \mathcal{X}_{x^{1}}^{1}\right] y_{1,1,1}+\left[-3 \mathcal{X}_{x^{1}}^{2}\right] y_{1,1,2}+\left[-4 \mathcal{X}_{y}^{1}\right] y_{1} y_{1,1,1}+ \\
& +\left[-\mathcal{X}_{y}^{2}\right] y_{2} y_{1,1,1}+\left[-3 \mathcal{X}_{y}^{2}\right] y_{1} y_{1,1,2} .
\end{aligned}\right.
$$

Inserting $\mathbf{Y}_{2}, \mathbf{Y}_{1,2}, \mathbf{Y}_{1,1,1}, \mathbf{Y}_{1}, \mathbf{Y}_{1,1}$ in the three Lie equations (8.73), (8.74), (8.75), replacing $y_{2}, y_{1,2}, y_{1,1,1}$ by the values they have on $\Delta_{\mathcal{E}_{5}}$, we get firstly five linear PDEs by picking the coefficients of cst., of $y_{1}$, of $\left(y_{1}\right)^{2}$, of $\left(y_{1}\right)^{3}$, of $\left(y_{1}\right)^{4}$ in (8.73):

$$
\left\{\begin{aligned}
0= & \mathcal{Y}_{x^{2}}, \\
0= & \mathcal{X}_{x^{2}}^{1}+\frac{1}{2} \mathcal{Y}_{x^{1}} \\
0= & \mathcal{Y}_{y}+\mathcal{X}_{x^{2}}^{2}-2 \mathcal{X}_{x^{1}}^{1}+\mathrm{r} \mathcal{Y}_{x^{1}}+\mathrm{s}^{*} \mathcal{Y}_{x^{1} x^{1}} \\
0=2 \mathcal{X}_{y}^{1}+\mathcal{X}_{x^{1}}^{2}+\mathrm{r} \mathcal{X}^{1}+\mathrm{r} \mathcal{X}^{2}+\mathrm{r} \mathcal{Y}_{x^{1}}+\mathrm{r} \mathcal{Y}_{y}+\mathrm{r} \mathcal{X}_{x^{1}}^{1}+ & \quad+\mathrm{r} \mathcal{Y}_{x^{1} x^{1}}+\mathrm{s}^{*} \mathcal{Y}_{x^{1} y}+\mathrm{s}^{*} \mathcal{X}_{x^{1} x^{1}}^{1} \\
& \\
& \quad+\mathrm{r} \mathcal{Y}_{x^{1} x^{1}}+\mathrm{r} \mathcal{Y}_{x^{1} y}+\mathrm{r} \mathcal{X}_{x^{1} x^{1}}^{1}+\mathrm{s}^{*} \mathcal{X}_{x^{1} x^{1}}^{2}+\mathrm{s}^{*} \mathcal{Y}_{y y}+\mathrm{s}^{*} \mathcal{X}_{x^{1} y}^{1}
\end{aligned}\right.
$$


secondly, we get three more linear PDEs by picking the coefficients of $\left(y_{1}\right)^{2}$, of $\left(y_{1}\right)^{3}$, of $\left(y_{1}\right)^{4}$ in (8.74):

$$
\left\{\begin{array}{r}
0=3 \mathcal{Y}_{x^{1} y}+\mathcal{X}_{x^{1} x^{2}}^{2}+4 \mathcal{X}_{x^{2} y}^{1}-2 \mathcal{X}_{x^{1} x^{1}}^{1}+\mathrm{r} \mathcal{X}^{1}+\mathrm{r} \mathcal{X}^{2}+\mathrm{r} \mathcal{Y}_{x^{1}}+\mathrm{r} \mathcal{Y}_{x^{1} x^{1}} \\
0=2 \mathcal{Y}_{y y}+2 \mathcal{X}_{x^{2} y}^{2}-6 \mathcal{X}_{x^{1} y}^{1}-\mathcal{X}_{x^{1} x^{1}}^{2}+\mathrm{r} \mathcal{X}^{1}+\mathrm{r} \mathcal{X}^{2}+\mathrm{r} \mathcal{Y}_{x^{1}}+\mathrm{r} \mathcal{Y}_{y}+\mathrm{r} \mathcal{X}_{x^{1}}^{1}+ \\
+\mathrm{r} \mathcal{Y}_{x^{1} x^{1}}+\mathrm{r} \mathcal{Y}_{x^{1} y}+\mathrm{r} \mathcal{X}_{x^{1} x^{1}}^{1} \\
0=4 \mathcal{X}_{y y}^{1}+3 \mathcal{X}_{x^{1} y}^{2}+\mathrm{r} \mathcal{X}^{1}+\mathrm{r} \mathcal{X}^{2}+\mathrm{r} \mathcal{Y}_{x^{1}}+\mathrm{r} \mathcal{Y}_{y}+\mathrm{r} \mathcal{X}_{x^{1}}^{1}+\mathrm{r} \mathcal{X}_{x^{1}}^{2}+\mathrm{r} \mathcal{X}_{y}^{1}+ \\
+\mathrm{r} \mathcal{Y}_{x^{1} x^{1}}+\mathrm{r} \mathcal{Y}_{x^{1} y}+\mathrm{r} \mathcal{X}_{x^{1} x^{1}}^{1}+\mathrm{r} \mathcal{X}_{x^{1} x^{1}}^{2}+\mathrm{r} \mathcal{Y}_{y y}+\mathrm{r} \mathcal{X}_{x^{1} y}^{1}
\end{array}\right.
$$

and thirdly, we get five more linear PDEs by picking the coefficients of cst., of $y_{1}$, of $y_{1,1}$, of $y_{1} y_{1,1}$, of $\left(y_{1}\right)^{2} y_{1,1}$ in (8.75)

$(8.83)$

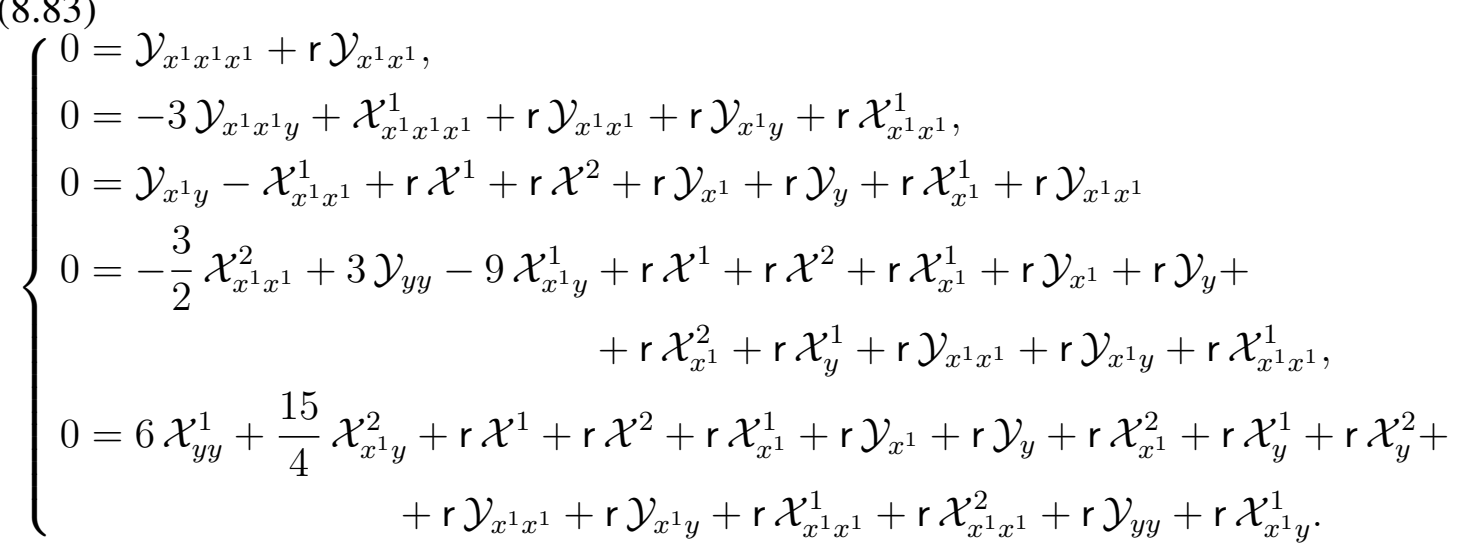

Proposition 8.84. Setting as initial conditions the ten specific differential coefficients (8.85)

$$
\begin{aligned}
\mathrm{P} & :=\mathrm{P}\left(\mathcal{X}^{1}, \mathcal{X}^{2}, \mathcal{Y}, \mathcal{X}_{y}^{1}, \mathcal{X}_{x^{2}}^{2}, \mathcal{Y}_{x^{1}}, \mathcal{Y}_{y}, \mathcal{X}_{x^{1} x^{2}}^{2}, \mathcal{Y}_{x^{1} x^{1}}, \mathcal{Y}_{y y}\right) \\
& =\mathrm{r} \mathcal{X}^{1}+\mathrm{r} \mathcal{X}^{2}+\mathrm{r} \mathcal{Y}+\mathrm{r} \mathcal{X}_{y}^{1}+\mathrm{r} \mathcal{X}_{x^{2}}^{2}+\mathrm{r} \mathcal{Y}_{x^{1}}+\mathrm{r} \mathcal{Y}_{y}+\mathrm{r} \mathcal{X}_{x^{1} x^{2}}^{2}+\mathrm{r} \mathcal{Y}_{x^{1} x^{1}}+\mathrm{r} \mathcal{Y}_{y y}
\end{aligned}
$$

it follows by cross differentiations and by linear substitutions from the Lie equations $(8.81)_{i}, i=1,2,3,4,5,(8.82)_{j}, j=1,2,3,(8.83)_{i}, i=1,2,3,4,5$, that $\mathcal{X}_{x^{1}}^{1}, \mathcal{X}_{x^{1}}^{2}$, $\mathcal{Y}_{x^{2}}, \mathcal{X}_{x^{2}}^{1}, \mathcal{X}_{y}^{2}, \mathcal{X}_{x^{1} y}^{1}, \mathcal{X}_{x^{2} x^{2}}^{2}, \mathcal{Y}_{x^{1} x^{2}}, \mathcal{X}_{x^{2} y}^{1}, \mathcal{X}_{x^{2} y}^{2}, \mathcal{Y}_{x^{1} y}, \mathcal{X}_{y y}^{1}, \mathcal{Y}_{x^{2} y}, \mathcal{X}_{x^{1} x^{1} x^{2}}^{2}, \mathcal{Y}_{x^{1} x^{1} x^{1}}, \mathcal{X}_{x^{1} x^{2} x^{2}}^{2}$ 
$\mathcal{Y}_{x^{1} x^{1} x^{2}}, \mathcal{X}_{x^{1} x^{2} y^{2}}^{2}, \mathcal{Y}_{x^{1} x^{1} y}, \mathcal{Y}_{x^{1} y y}, \mathcal{Y}_{x^{2} y y}, \mathcal{Y}_{y y y}$ are uniquely determined as linear combinations of $\left(\mathcal{X}^{1}, \mathcal{X}^{2}, \mathcal{Y}, \mathcal{X}_{y}^{1}, \mathcal{X}_{x^{2}}^{2}, \mathcal{Y}_{x^{1}}, \mathcal{Y}_{y}, \mathcal{X}_{x^{1} x^{1}}^{2}, \mathcal{Y}_{x^{1} x^{1}}, \mathcal{Y}_{y y}\right)$, namely:

$$
\left\{\begin{array}{ccc}
\mathcal{X}_{x^{1}}^{1} \stackrel{1}{=} \mathrm{P}, & \mathcal{X}_{x^{1}}^{2} \stackrel{2}{=} \mathrm{P}, & \mathcal{Y}_{x^{2}} \stackrel{3}{=} \mathrm{P}, \\
\mathcal{X}_{x^{2}}^{1} \stackrel{4}{=} \mathrm{P}, & \mathcal{X}_{y}^{2} \stackrel{5}{=} \mathrm{P}, & \\
\mathcal{X}_{x^{1} y}^{1} \stackrel{6}{=} \mathrm{P}, & \mathcal{X}_{x^{2} x^{2}}^{2} \stackrel{7}{=} \mathrm{P}, & \mathcal{Y}_{x^{1} x^{2}} \stackrel{8}{=} \mathrm{P}, \\
\mathcal{X}_{x^{2} y}^{1} \stackrel{9}{=} \mathrm{P}, & \mathcal{X}_{x^{2} y}^{2} \stackrel{10}{=} \mathrm{P}, & \mathcal{Y}_{x^{1} y} \stackrel{11}{=} \mathrm{P}, \\
\mathcal{X}_{y y}^{1} \stackrel{12}{=} \mathrm{P}, & \mathcal{Y}_{x^{2} y} \stackrel{13}{=} \mathrm{P}, \\
& \mathcal{X}_{x^{1} x^{1} x^{2}}^{2} \stackrel{14}{=} \mathrm{P}, & \mathcal{Y}_{x^{1} x^{1} x^{1}} \stackrel{15}{=} \mathrm{P}, \\
& \mathcal{X}_{x^{1} x^{2} x^{2}} \stackrel{16}{=} \mathrm{P}, & \mathcal{Y}_{x^{1} x^{1} x^{2}} \stackrel{17}{=} \mathrm{P}, \\
& \mathcal{X}_{x^{1} x^{2} y}^{2} \stackrel{18}{=} \mathrm{P}, & \mathcal{Y}_{x^{1} x^{1} y} \stackrel{19}{=} \mathrm{P}, \\
& & \mathcal{Y}_{x^{1} y y} \stackrel{20}{=} \mathrm{P}, \\
& \mathcal{Y}_{x^{2} y y} \stackrel{21}{=} \mathrm{P}, \\
& \mathcal{Y}_{y y y} \stackrel{22}{=} \mathrm{P} .
\end{array}\right.
$$

Then the expressions $\mathrm{P}$ are stable under differentiation with respect to $x^{1}$, to $x^{2}$, to $y$ and moreover, all other, higher order partial derivatives of $\mathcal{X}^{1}$, of $\mathcal{X}^{2}$, of $\mathcal{Y}$ may be expressed as $\mathrm{P}\left(\mathcal{X}^{1}, \mathcal{X}^{2}, \mathcal{Y}, \mathcal{X}_{y}^{1}, \mathcal{X}_{x^{2}}^{2}, \mathcal{Y}_{x^{1}}, \mathcal{Y}_{y}, \mathcal{X}_{x^{1} x^{2}}^{2}, \mathcal{Y}_{x^{1} x^{1}}, \mathcal{Y}_{y y}\right)$.

Corollary 8.87. Every infinitesimal Lie symmetry of the PDE system $\left(\mathcal{E}_{5}\right)$ is uniquely determined by the ten initial Taylor coefficients

$$
\mathcal{X}^{1}(0), \mathcal{X}^{2}(0), \mathcal{Y}(0), \mathcal{X}_{y}^{1}(0), \mathcal{X}_{x^{2}}^{2}(0), \mathcal{Y}_{x^{1}}(0), \mathcal{Y}_{y}(0), \mathcal{X}_{x^{1} x^{2}}^{2}(0), \mathcal{Y}_{x^{1} x^{1}}(0), \mathcal{Y}_{y y}(0)
$$

Proof of the proposition. At first, $(8.83)_{1}$ yields $(8.86)_{15} ;(8.81)_{1}$ yields $(8.86)_{3}$; differentiating $(8.81)_{1}$ with respect to $x^{1}$ yields $(8.86)_{8}$; differentiating $(8.81)_{1}$ with respect to $y$ yields $(8.86)_{13}$; differentiating $(8.81)_{1}$ with respect to $x^{1} x^{1}$ yields $(8.86)_{17}$; and differentiating $(8.81)_{1}$ with respect to yy yields $(8.86)_{21}$. Also, rewriting $(8.81)_{2}$ as

$$
\mathcal{X}_{x^{2}}^{1}=-\frac{1}{2} \mathcal{Y}_{x^{1}}
$$

we get $(8.86)_{4}$; and rewriting $(8.81)_{3}$ as

$$
\mathcal{X}_{x^{1}}^{1}=\frac{1}{2} \mathcal{X}_{x^{2}}^{2}+\frac{1}{2} \mathcal{Y}_{y}+\mathrm{r} \mathcal{Y}_{x^{1}}+\mathrm{s}^{*} \mathcal{Y}_{x^{1} x^{1}}
$$

we get $(8.86)_{1}$.

Next, differentiating $(8.81)_{2}$ with respect to $x^{1}$ and $(8.81)_{3}$ with respect to $x^{2}$, we get, taking account of $0=\mathcal{Y}_{x^{2} y}=\mathcal{Y}_{x^{1} x^{2}}=\mathcal{Y}_{x^{1} x^{1} x^{2}}$, replacing $\mathcal{X}_{x^{1} x^{2}}$ by $-\frac{1}{2} \mathcal{Y}_{x^{1} x^{1}}$ and solving for $\mathcal{X}_{x^{2} x^{2}}^{2}$ :

$$
\begin{aligned}
0 & =\mathcal{X}_{x^{1} x^{2}}^{1}+\frac{1}{2} \mathcal{Y}_{x^{1} x^{1}}, \\
\mathcal{X}_{x^{2} x^{2}}^{2} & =-\left(1+\mathrm{s}_{x^{2}}^{*}\right) \mathcal{Y}_{x^{1} x^{1}}+\mathrm{r} \mathcal{Y}_{x^{1}} .
\end{aligned}
$$

This is $(8.86)_{7}$. Differentiating $(8.91)_{2}$ with respect to $x^{1}$, taking account of $(8.83)_{1}$, we get $(8.86)_{16}$ :

$$
\mathcal{X}_{x^{1} x^{2} x^{2}}^{2}=r \mathcal{Y}_{x^{1}}+r \mathcal{Y}_{x^{1} x^{1}}
$$


We then replace $\mathcal{X}_{x^{1}}^{1}$ from $(8.90)$ in $(8.81)_{4}$ :

$$
\begin{aligned}
0=\mathcal{X}_{x^{1}}^{2}+2 \mathcal{X}_{y}^{1} & +r \mathcal{X}^{1}+r \mathcal{X}^{2}+r \mathcal{X}_{x^{2}}^{2}+r \mathcal{Y}_{x^{1}}+r \mathcal{Y}_{y}+ \\
& +r \mathcal{Y}_{x^{1} x^{1}}+\mathrm{s}^{*} \mathcal{Y}_{x^{1} y}+\mathrm{s}^{*} \mathcal{X}_{x^{1} x^{1}}^{1} .
\end{aligned}
$$

We differentiate this equation with respect to $x^{2}$, knowing $\mathcal{Y}_{x^{2}}=0$ :

$$
\begin{aligned}
0=\mathcal{X}_{x^{1} x^{2}}^{2}+2 \mathcal{X}_{x^{2} y}^{1} & +\mathrm{r} \mathcal{X}^{1}+\mathrm{r} \mathcal{X}_{x^{2}}^{1}+\mathrm{r} \mathcal{X}^{2}+\mathrm{r} \mathcal{X}_{x^{2}}^{2}+\mathrm{r} \mathcal{X}_{x^{2} x^{2}}^{2}+\mathrm{r} \mathcal{Y}_{x^{1}}+\mathrm{r} \mathcal{Y}_{y}+ \\
& +\mathrm{r} \mathcal{Y}_{x^{1} x^{1}}+\mathrm{s}_{x^{2}}^{*} \mathcal{Y}_{x^{1} y}+\mathrm{s}_{x^{2}}^{*} \mathcal{X}_{x^{1} x^{1}}^{1}+\mathrm{s}^{*} \mathcal{X}_{x^{1} x^{1} x^{2}} .
\end{aligned}
$$

We replace: $\mathcal{X}_{x^{2}}^{1}$ from (8.89); $\mathcal{X}_{x^{2} x^{2}}^{2}$ from $(8.91)_{2}$; we differentiate $(8.81)_{2}$ with respect to $x^{1} x^{1}$ to replace $\mathcal{X}_{x^{1} x^{1} x^{2}}^{1}$ by $r \mathcal{Y}_{x^{1} x^{1}}$, thanks to $(8.83)_{1}$; and we reorganize:

(8.95) $2 \mathcal{X}_{x^{2} y}^{1}+s_{x^{2}}^{*} \mathcal{Y}_{x^{1} y}+s_{x^{2}}^{*} \mathcal{X}_{x^{1} x^{1}}^{1}=-\mathcal{X}_{x^{1} x^{2}}^{2}+\mathrm{r} \mathcal{X}^{1}+\mathrm{r} \mathcal{X}^{2}+\mathrm{r} \mathcal{X}_{x^{2}}^{2}+\mathrm{r} \mathcal{Y}_{x^{1}}+\mathrm{r} \mathcal{Y}_{y}+\mathrm{r} \mathcal{Y}_{x^{1} x^{1}}$

We differentiate $(8.81)_{2}$ with respect to $y$ and $(8.81)_{3}$ with respect to $x^{1}$ :

$$
\begin{aligned}
& \mathcal{X}_{x^{2} y}^{1}+\frac{1}{2} \mathcal{Y}_{x^{1} y}=0 \\
& \mathcal{Y}_{x^{1} y}-2 \mathcal{X}_{x^{1} x^{1}}^{1}=-\mathcal{X}_{x^{1} x^{2}}^{2}+r \mathcal{Y}_{x^{1}}+r \mathcal{Y}_{x^{1} x^{1}}
\end{aligned}
$$

For the three unknowns $\mathcal{X}_{x^{1} x^{1}}^{1}, \mathcal{Y}_{x^{1} y}, \mathcal{X}_{x^{2} y}^{1}$, we solve the three equations (8.95), (8.96) $)_{1}$, $(8.96)_{2}$, reminding $\mathrm{s}_{x^{2}}^{*}(0)=0$ :

$$
\begin{aligned}
\mathcal{X}_{x^{1} x^{1}}^{1} & =\mathrm{r} \mathcal{X}^{1}+\mathrm{r} \mathcal{X}^{2}+\mathrm{r} \mathcal{X}_{x^{2}}^{2}+\mathrm{r} \mathcal{Y}_{x^{1}}+\mathrm{r} \mathcal{Y}_{y}+\mathrm{r} \mathcal{Y}_{x^{1} x^{1}}+\mathrm{r} \mathcal{X}_{x^{1} x^{2}}^{2}, \\
\mathcal{Y}_{x^{1} y} & =\mathrm{r} \mathcal{X}^{1}+\mathrm{r} \mathcal{X}^{2}+\mathrm{r} \mathcal{X}_{x^{2}}^{2}+r \mathcal{Y}_{x^{1}}+\mathrm{r} \mathcal{Y}_{y}+\mathrm{r} \mathcal{Y}_{x^{1} x^{1}}+\mathrm{r} \mathcal{X}_{x^{1} x^{2}}^{2}, \\
\mathcal{X}_{x^{2} y}^{1} & =\mathrm{r} \mathcal{X}^{1}+\mathrm{r} \mathcal{X}^{2}+\mathrm{r} \mathcal{X}_{x^{2}}^{2}+r \mathcal{Y}_{x^{1}}+r \mathcal{Y}_{y}+r \mathcal{Y}_{x^{1} x^{1}}+r \mathcal{X}_{x^{1} x^{2}}^{2}
\end{aligned}
$$

We get (8.86) $)_{11}$ and $(8.86)_{9}$.

Thus, we may replace $\mathcal{X}_{x^{1} x^{1}}^{1}$ and $\mathcal{Y}_{x^{1} y}$ in $(8.81)_{4}$ to get $(8.86)_{2}$ :

$$
\mathcal{X}_{x^{1}}^{2}=-2 \mathcal{X}_{y}^{1}+\mathrm{r} \mathcal{X}^{1}+\mathrm{r} \mathcal{X}^{2}+\mathrm{r} \mathcal{X}_{x^{2}}^{2}+\mathrm{r} \mathcal{Y}_{x^{1}}+\mathrm{r} \mathcal{Y}_{y}+\mathrm{r} \mathcal{Y}_{x^{1} x^{1}}+\mathrm{r} \mathcal{X}_{x^{1} x^{2}}^{2}
$$

Next, we differentiate $(8.83)_{3}$ with respect to $x^{1}$ and we replace: $\mathcal{X}_{x^{1}}^{1}$ from (8.90); $\mathcal{X}_{x^{1}}^{2}$ from (8.98); $\mathcal{Y}_{x^{1} y}$ from $(8.97)_{2} ; \mathcal{X}_{x^{1} x^{1}}^{1}$ from $(8.97)_{1} ; \mathcal{Y}_{x^{1} x^{1} x^{1}}$ from $(8.83)_{1}$; and we compare with $(8.83)_{2}$; we differentiate $(8.96)_{1}$ with respect to $x^{1}$ and $(8.96)_{2}$ with respect to $x^{1}$; solving, we obtain four new relations:

$$
\begin{aligned}
\mathcal{X}_{x^{1} x^{1} x^{1}}^{1} & =\mathrm{r} \mathcal{X}^{1}+\mathrm{r} \mathcal{X}^{2}+\mathrm{r} \mathcal{X}_{x^{2}}^{2}+\mathrm{r} \mathcal{Y}_{x^{1}}+\mathrm{r} \mathcal{Y}_{y}+\mathrm{r} \mathcal{Y}_{x^{1} x^{1}}+\mathrm{r} \mathcal{X}_{x^{1} x^{2}}^{2}, \\
\mathcal{Y}_{x^{1} x^{1} y} & =\mathrm{r} \mathcal{X}^{1}+\mathrm{r} \mathcal{X}^{2}+\mathrm{r} \mathcal{X}_{x^{2}}^{2}+\mathrm{r} \mathcal{Y}_{x^{1}}+\mathrm{r} \mathcal{Y}_{y}+\mathrm{r} \mathcal{Y}_{x^{1} x^{1}}+\mathrm{r} \mathcal{X}_{x^{1} x^{2}}^{2}, \\
\mathcal{X}_{x^{1} x^{2} y}^{2} & =\mathrm{r} \mathcal{X}^{1}+\mathrm{r} \mathcal{X}^{2}+\mathrm{r} \mathcal{X}_{x^{2}}^{2}+\mathrm{r} \mathcal{Y}_{x^{1}}+\mathrm{r} \mathcal{Y}_{y}+\mathrm{r} \mathcal{Y}_{x^{1} x^{1}}+\mathrm{r} \mathcal{X}_{x^{1} x^{2}}^{2}, \\
\mathcal{X}_{x^{1} x^{1} x^{2}}^{2} & =\mathrm{r} \mathcal{X}^{1}+\mathrm{r} \mathcal{X}^{2}+\mathrm{r} \mathcal{X}_{x^{2}}^{2}+\mathrm{r} \mathcal{Y}_{x^{1}}+\mathrm{r} \mathcal{Y}_{y}+\mathrm{r} \mathcal{Y}_{x^{1} x^{1}}+\mathrm{r} \mathcal{X}_{x^{1} x^{2}}^{2}
\end{aligned}
$$

We get $(8.86)_{19}$ and $(8.86)_{14}$.

Next, in $(8.81)_{5}$, we replace: $\mathcal{X}_{x^{1}}^{1}$ from $(8.90) ; \mathcal{X}_{x^{1}}^{2}$ from $(8.98) ; \mathcal{Y}_{x^{1} y}$ from $(8.97)_{2}$; we get:

$$
\begin{aligned}
\mathcal{X}_{y}^{2}= & \mathrm{r} \mathcal{X}^{1}+\mathrm{r} \mathcal{X}^{2}+\mathrm{r} \mathcal{X}_{y}^{1}+\mathrm{r} \mathcal{X}_{x^{2}}^{2}+\mathrm{r} \mathcal{Y}_{x^{1}}+\mathrm{r} \mathcal{Y}_{y}+\mathrm{r} \mathcal{Y}_{x^{1} x^{1}}+ \\
& +\mathrm{s}^{*} \mathcal{X}_{x^{1} x^{1}}^{2}+\mathrm{s}^{*} \mathcal{Y}_{y y}+\mathrm{s}^{*} \mathcal{X}_{x^{1} y}^{1} .
\end{aligned}
$$

We differentiate (8.98) with respect to $x^{1}$ and we replace: $\mathcal{X}_{x^{1}}^{1}$ from (8.90); $\mathcal{X}_{x^{1}}^{2}$ from (8.98); $\mathcal{Y}_{x^{1} y}$ from $(8.97)_{2} ; \mathcal{Y}_{x^{1} x^{1} x^{1}}$ from $(8.83)_{1} ; \mathcal{X}_{x^{1} x^{1} x^{2}}^{2}$ from $(8.99)_{4}$; we get:

$(8.101) \mathcal{X}_{x^{1} x^{1}}^{2}+2 \mathcal{X}_{x^{1} y}^{1}=\mathrm{r} \mathcal{X}^{1}+\mathrm{r} \mathcal{X}^{2}+\mathrm{r} \mathcal{X}_{y}^{1}+\mathrm{r} \mathcal{X}_{x^{2}}^{2}+\mathrm{r} \mathcal{Y}_{x^{1}}+\mathrm{r} \mathcal{Y}_{y}+\mathrm{r} \mathcal{Y}_{x^{1} x^{1}}+\mathrm{r} \mathcal{X}_{x^{1} x^{2}}^{2}$ 
In $(8.82)_{2}$, we replace: $\mathcal{X}_{x^{1}}^{1}$ from $(8.90) ; \mathcal{X}_{x^{1} x^{1}}^{1}$ from $(8.97)_{1} ; \mathcal{Y}_{x^{1} y}$ from $(8.97)_{2}$; and we reorganize:

(8.102)

$2 \mathcal{X}_{x^{2} y}^{2}-6 \mathcal{X}_{x^{1} y}^{1}-\mathcal{X}_{x^{1} x^{1}}^{2}=-2 \mathcal{Y}_{y y}+\mathrm{r} \mathcal{X}^{1}+\mathrm{r} \mathcal{X}^{2}+\mathrm{r} \mathcal{X}_{x^{2}}^{2}+\mathrm{r} \mathcal{Y}_{x^{1}}+\mathrm{r} \mathcal{Y}_{y}+\mathrm{r} \mathcal{Y}_{x^{1} x^{1}}+\mathrm{r} \mathcal{X}_{x^{1} x^{2}}^{2}$

Differentiating $(8.81)_{3}$ with respect to $y$, we replace: $\mathcal{Y}_{x^{1} y}$ from $(8.97)_{2} ; \mathcal{Y}_{x^{1} x^{1} y}$ from $(8.99)_{2}$; and we reorganize:

$(8.103) \mathcal{X}_{x^{2} y}^{2}-2 \mathcal{X}_{x^{1} y}^{1}=-\mathcal{Y}_{y y}+\mathrm{r} \mathcal{X}^{1}+\mathrm{r} \mathcal{X}^{2}+\mathrm{r} \mathcal{X}_{x^{2}}^{2}+\mathrm{r} \mathcal{Y}_{x^{1}}+\mathrm{r} \mathcal{Y}_{y}+\mathrm{r} \mathcal{Y}_{x^{1} x^{1}}+\mathrm{r} \mathcal{X}_{x^{1} x^{2}}^{2}$

For the three unknowns $\mathcal{X}_{x^{1} x^{1}}^{2}, \mathcal{X}_{x^{1} y}^{1}, \mathcal{X}_{x^{2} y}^{2}$, we then solve the four equations (8.101), (8.102), (8.103), (8.83) (in which we replace: $\mathcal{X}_{x^{1}}^{1}$ from (8.90); $\mathcal{X}_{x^{1}}^{2}$ from (8.98); $\mathcal{Y}_{x^{1} y}$ from $(8.97)_{2} ; \mathcal{X}_{x^{1} x^{1}}^{1}$ from $\left.(8.97)_{1}\right)$ :

(8.104)

$$
\begin{aligned}
\mathcal{X}_{x^{1} x^{1}}^{2} & =r \mathcal{X}^{1}+r \mathcal{X}^{2}+r \mathcal{X}_{y}^{1}+r \mathcal{X}_{x^{2}}^{2}+r \mathcal{Y}_{x^{1}}+r \mathcal{Y}_{y}+r \mathcal{Y}_{x^{1} x^{1}}+\mathrm{r} \mathcal{X}_{x^{1} x^{2}}^{2}+r \mathcal{Y}_{y y}, \\
\mathcal{X}_{x^{1} y}^{1} & =r \mathcal{X}^{1}+r \mathcal{X}^{2}+r \mathcal{X}_{y}^{1}+r \mathcal{X}_{x^{2}}^{2}+r \mathcal{Y}_{x^{1}}+r \mathcal{Y}_{y}+r \mathcal{Y}_{x^{1} x^{1}}+r \mathcal{X}_{x^{1} x^{2}}^{2}+r \mathcal{Y}_{y y}, \\
\mathcal{X}_{x^{2} y}^{2} & =r \mathcal{X}^{1}+r \mathcal{X}^{2}+r \mathcal{X}_{y}^{1}+r \mathcal{X}_{x^{2}}^{2}+r \mathcal{Y}_{x^{1}}+r \mathcal{Y}_{y}+r \mathcal{Y}_{x^{1} x^{1}}+r \mathcal{X}_{x^{1} x^{2}}^{2}+r \mathcal{Y}_{y y} .
\end{aligned}
$$

We get $(8.86)_{6}$ and (8.86) $)_{10}$. Replacing then $\mathcal{X}_{x^{1} x^{1}}^{2}, \mathcal{X}_{x^{1} y}^{1}$ in (8.100) gives

(8.105) $\mathcal{X}_{y}^{2}=r \mathcal{X}^{1}+r \mathcal{X}^{2}+r \mathcal{X}_{y}^{1}+r \mathcal{X}_{x^{2}}^{2}+r \mathcal{Y}_{x^{1}}+r \mathcal{Y}_{y}+r \mathcal{Y}_{x^{1} x^{1}}+r \mathcal{X}_{x^{1} x^{2}}^{2}+r \mathcal{Y}_{y y}$

This is $(8.86)_{5}$.

Next, we differentiate (8.103) with respect to $x^{1}$ and we replace: $\mathcal{X}_{x^{1}}^{1}$ from (8.90); $\mathcal{X}_{x^{1}}^{2}$ from (8.98); $\mathcal{Y}_{x^{1} y}$ from $(8.97)_{2} ; \mathcal{Y}_{x^{1} x^{1} x^{1}}$ from $(8.83)_{1} ; \mathcal{X}_{x^{1} x^{1} x^{2}}^{2}$ from (8.99) $)_{4}$; we get:

(8.106) $\mathcal{Y}_{x^{1} y y}+\mathcal{X}_{x^{1} x^{2} y}^{2}-2 \mathcal{X}_{x^{1} x^{1} y}^{1}=r \mathcal{X}^{1}+\mathrm{r} \mathcal{X}^{2}+\mathrm{r} \mathcal{X}_{x^{2}}^{2}+\mathrm{r} \mathcal{Y}_{x^{1}}+\mathrm{r} \mathcal{Y}_{y}+\mathrm{r} \mathcal{Y}_{x^{1} x^{1}}+\mathrm{r} \mathcal{X}_{x^{1} x^{2}}^{2}$

Also, we differentiate $(8.83)_{3}$ with respect to $y$ and we replace: $\mathcal{X}_{y}^{2}$ from $(8.105) ; \mathcal{Y}_{x^{1} y}$ from $(8.97)_{2} ; \mathcal{X}_{x^{1} y}^{1}$ from $(8.104)_{2} ; \mathcal{Y}_{x^{1} x^{1} y}$ from $(8.99)_{2}$; we get:

(8.107) $\mathcal{Y}_{x^{1} y y}-\mathcal{X}_{x^{1} x^{1} y}^{1}=r \mathcal{X}^{1}+r \mathcal{X}^{2}+r \mathcal{X}_{y}^{1}+r \mathcal{X}_{x^{2}}^{2}+r \mathcal{Y}_{x^{1}}+r \mathcal{Y}_{y}+r \mathcal{Y}_{x^{1} x^{1}}+\mathrm{r} \mathcal{X}_{x^{1} x^{2}}^{2}+\mathrm{r} \mathcal{Y}_{y y}$

Also, we replace in (8.82) $: \mathcal{X}_{x^{1}}^{1}$ from (8.90); $\mathcal{X}_{x^{1}}^{2}$ from (8.98); $\mathcal{Y}_{x^{1} y}$ from $(8.97)_{2} ; \mathcal{X}_{x^{1} x^{1}}^{1}$ from $(8.97)_{1} ; \mathcal{X}_{x^{1} x^{1}}^{2}$ from $(8.104)_{1} ; \mathcal{X}_{x^{1} y}^{1}$ from $(8.104)_{2}$; we get:

$(8.108) 4 \mathcal{X}_{y y}^{1}+3 \mathcal{X}_{x^{1} y}^{2}=r \mathcal{X}^{1}+r \mathcal{X}^{2}+r \mathcal{X}_{y}^{1}+r \mathcal{X}_{x^{2}}^{2}+r \mathcal{Y}_{x^{1}}+r \mathcal{Y}_{y}+r \mathcal{Y}_{x^{1} x^{1}}+\mathrm{r} \mathcal{X}_{x^{1} x^{2}}^{2}+\mathrm{r} \mathcal{Y}_{y y}$

We differentiate this equation with respect to $x^{2}$ and we replace: $4 \mathcal{X}_{x^{2} y y}^{1}$ by $-2 \mathcal{Y}_{x^{1} y y}$ from (8.89); $\mathcal{X}_{x^{2}}^{1}$ from (8.98); $\mathcal{X}_{x^{2} y}^{1}$ from $(8.97)_{3}$; (notice $0=\mathcal{Y}_{x^{1} x^{2}}=\mathcal{Y}_{x^{2} y}$ ); $\mathcal{X}_{x^{2} x^{2}}^{2}$ from $(8.91)_{2} ; \mathcal{X}_{x^{1} x^{2} x^{2}}^{2}$ from (8.92); we get:

$-2 \mathcal{Y}_{x^{1} y y}+3 \mathcal{X}_{x^{1} x^{2} y}^{2}=r \mathcal{X}^{1}+r \mathcal{X}^{2}+r \mathcal{X}_{y}^{1}+r \mathcal{X}_{x^{2}}^{2}+r \mathcal{Y}_{x^{1}}+r \mathcal{Y}_{y}+r \mathcal{Y}_{x^{1} x^{1}}+r \mathcal{X}_{x^{1} x^{2}}^{2}+r \mathcal{Y}_{y y}$

For the three unknowns $\mathcal{X}_{x^{1} x^{1} y}^{1}, \mathcal{Y}_{x^{1} y y}, \mathcal{X}_{x^{1} x^{2} y}^{2}$, we solve the three equations (8.106), (8.107), (8.108); we get:

(8.110)

$$
\begin{aligned}
\mathcal{X}_{x^{1} x^{1} y}^{1} & =r \mathcal{X}^{1}+r \mathcal{X}^{2}+r \mathcal{X}_{y}^{1}+r \mathcal{X}_{x^{2}}^{2}+r \mathcal{Y}_{x^{1}}+r \mathcal{Y}_{y}+r \mathcal{Y}_{x^{1} x^{1}}+r \mathcal{X}_{x^{1} x^{2}}^{2}+r \mathcal{Y}_{y y}, \\
\mathcal{Y}_{x^{1} y y} & =r \mathcal{X}^{1}+r \mathcal{X}^{2}+r \mathcal{X}_{y}^{1}+r \mathcal{X}_{x^{2}}^{2}+r \mathcal{Y}_{x^{1}}+r \mathcal{Y}_{y}+r \mathcal{Y}_{x^{1} x^{1}}+r \mathcal{X}_{x^{1} x^{2}}^{2}+r \mathcal{Y}_{y y}, \\
\mathcal{X}_{x^{1} x^{2} y}^{2} & =r \mathcal{X}^{1}+r \mathcal{X}^{2}+r \mathcal{X}_{y}^{1}+r \mathcal{X}_{x^{2}}^{2}+r \mathcal{Y}_{x^{1}}+r \mathcal{Y}_{y}+r \mathcal{Y}_{x^{1} x^{1}}+r \mathcal{X}_{x^{1} x^{2}}^{2}+r \mathcal{Y}_{y y}
\end{aligned}
$$

We get $(8.86)_{20}$ and $(8.86)_{18}$.

Next, in (8.93), we replace: $\mathcal{Y}_{x^{1} y}$ from $(8.97)_{2} ; \mathcal{X}_{x^{1} x^{1}}^{1}$ from (8.97) ; we get:

$$
\mathcal{X}_{x^{1}}^{2}+2 \mathcal{X}_{y}^{1}=r \mathcal{X}^{1}+r \mathcal{X}^{2}+r \mathcal{X}_{x^{2}}^{2}+r \mathcal{Y}_{x^{1}}+r \mathcal{Y}_{y}+r \mathcal{Y}_{x^{1} x^{1}}+r \mathcal{X}_{x^{1} x^{2}}^{2} .
$$


We differentiate this equation with respect to $y$ and we replace: $\mathcal{X}_{y}^{2}$ from (8.105); $\mathcal{X}_{x^{2} y}^{2}$ from $(8.104)_{3} ; \mathcal{Y}_{x^{1} y}$ from $(8.97)_{2} ; \mathcal{Y}_{x^{1} x^{1} y}$ from $(8.99)_{2} ; \mathcal{X}_{x^{1} x^{2} y}^{2}$ from $(8.99)_{3}$; we get:

(8.112) $\mathcal{X}_{x^{1} y}^{2}+2 \mathcal{X}_{y y}^{1}=r \mathcal{X}^{1}+r \mathcal{X}^{2}+r \mathcal{X}_{y}^{1}+r \mathcal{X}_{x^{2}}^{2}+r \mathcal{Y}_{x^{1}}+r \mathcal{Y}_{y}+r \mathcal{Y}_{x^{1} x^{1}}+\mathrm{r} \mathcal{X}_{x^{1} x^{2}}^{2}+\mathrm{r} \mathcal{Y}_{y y}$

For the two unknowns $\mathcal{X}_{y y}^{1}$ and $\mathcal{X}_{x^{1} y}^{2}$, we solve the two equations (8.108) and (8.112); we get:

$$
\begin{aligned}
\mathcal{X}_{y y}^{1} & =r \mathcal{X}^{1}+r \mathcal{X}^{2}+r \mathcal{X}_{y}^{1}+r \mathcal{X}_{x^{2}}^{2}+r \mathcal{Y}_{x^{1}}+r \mathcal{Y}_{y}+r \mathcal{Y}_{x^{1} x^{1}}+\mathrm{r} \mathcal{X}_{x^{1} x^{2}}^{2}+r \mathcal{Y}_{y y} \\
\mathcal{X}_{x^{1} y}^{2} & =r \mathcal{X}^{1}+r \mathcal{X}^{2}+r \mathcal{X}_{y}^{1}+r \mathcal{X}_{x^{2}}^{2}+r \mathcal{Y}_{x^{1}}+r \mathcal{Y}_{y}+r \mathcal{Y}_{x^{1} x^{1}}+\mathrm{r} \mathcal{X}_{x^{1} x^{2}}^{2}+r \mathcal{Y}_{y y}
\end{aligned}
$$

We get $(8.86)_{12}$.

Next, we differentiate $(8.113)_{1}$ with respect to $x^{1}$ and we replace: $\mathcal{X}_{x^{1}}^{1}, \mathcal{X}_{x^{1}}^{2}, \mathcal{X}_{x^{1} y}^{1}, \mathcal{Y}_{x^{1} y}$, $\mathcal{Y}_{x^{1} x^{1} x^{1}}, \mathcal{X}_{x^{1} x^{1} x^{2}}^{2}, \mathcal{Y}_{x^{1} y y}$; we get:

(8.114) $\mathcal{X}_{x^{1} y y}^{1}=r \mathcal{X}^{1}+r \mathcal{X}^{2}+r \mathcal{X}_{y}^{1}+r \mathcal{X}_{x^{2}}^{2}+r \mathcal{Y}_{x^{1}}+r \mathcal{Y}_{y}+r \mathcal{Y}_{x^{1} x^{1}}+r \mathcal{X}_{x^{1} x^{2}}^{2}+r \mathcal{Y}_{y y}$

Also, we differentiate $(8.113)_{2}$ with respect to $x^{1}$ and we replace:

(8.115) $\mathcal{X}_{x^{1} x^{1} y}^{2}=\mathrm{r} \mathcal{X}^{1}+\mathrm{r} \mathcal{X}^{2}+\mathrm{r} \mathcal{X}_{y}^{1}+\mathrm{r} \mathcal{X}_{x^{2}}^{2}+\mathrm{r} \mathcal{Y}_{x^{1}}+\mathrm{r} \mathcal{Y}_{y}+\mathrm{r} \mathcal{Y}_{x^{1} x^{1}}+\mathrm{r} \mathcal{X}_{x^{1} x^{2}}^{2}+\mathrm{r} \mathcal{Y}_{y y}$

Also, we differentiate $(8.83)_{4}$ with respect to $y$; we replace $\mathcal{X}_{x^{1} y y}^{1}$ from (8.114), we replace $\mathcal{X}_{x^{1} x^{1} y}^{2}$ from (8.115); and we achieve other evident replacements; we get:

(8.116) $\mathcal{Y}_{y y y}=r \mathcal{X}^{1}+r \mathcal{X}^{2}+r \mathcal{X}_{y}^{1}+r \mathcal{X}_{x^{2}}^{2}+r \mathcal{Y}_{x^{1}}+r \mathcal{Y}_{y}+r \mathcal{Y}_{x^{1} x^{1}}+r \mathcal{X}_{x^{1} x^{2}}^{2}+r \mathcal{Y}_{y y}$.

This is $(8.96)_{22}$, which completes the proof.

Theorem 8.117. The bound $\operatorname{dim} \mathfrak{S Y M}\left(\mathcal{E}_{5}\right) \leqslant 10$ is attained if and only if $\left(\mathcal{E}_{5}\right)$ is equivalent, through a diffeomorphism $\left(x^{1}, x^{2}, y\right) \longmapsto\left(X^{1}, X^{2}, Y\right)$, to the model system

$$
Y_{X^{2}}=0, \quad Y_{X^{1} X^{1} X^{1}}=0 .
$$

Proof. Firstly, setting $\mathrm{r}=\mathrm{s}^{*}=0$ everywhere, the solution to (8.81), (8.82), (8.83) is

$$
\begin{aligned}
\mathcal{X}^{1} & =k+(c+j) x^{1}-b x^{2}-h y+e x^{1} x^{1}-d x^{1} x^{2}+f x^{1} y-e x^{2} y, \\
\mathcal{X}^{2} & =g+2 h x^{1}+2 j x^{2}-d x^{2} x^{2}+2 e x^{1} x^{2}-f x^{1} x^{1}, \\
\mathcal{Y} & =a+2 b x^{1}+2 c y+d x^{1} x^{1}+2 e x^{1} y+f y y,
\end{aligned}
$$

where $a, b, c, d, e, f, g, h, j, k \in \mathbb{K}$ are arbitrary. Computing the third prolongations of the ten vector fields

(8.120)

$$
\begin{aligned}
& \frac{\partial}{\partial x^{1}}, \quad \frac{\partial}{\partial x^{2}}, \quad \frac{\partial}{\partial y}, \\
& -x^{2} \frac{\partial}{\partial x^{1}}+2 x^{1} \frac{\partial}{\partial y}, \quad x^{1} \frac{\partial}{\partial x^{1}}+2 y \frac{\partial}{\partial y}, \quad x^{1} \frac{\partial}{\partial x^{1}}+2 x^{2} \frac{\partial}{\partial x^{2}}, \quad-y \frac{\partial}{\partial x^{1}}+2 x^{1} \frac{\partial}{\partial x^{2}}, \\
& -x^{1} x^{2} \frac{\partial}{\partial x^{1}}-x^{2} x^{2} \frac{\partial}{\partial x^{2}}+x^{1} x^{1} \frac{\partial}{\partial y}, \quad\left(x^{1} x^{1}-x^{2} y\right) \frac{\partial}{\partial x^{1}}+2 x^{1} x^{2} \frac{\partial}{\partial x^{2}}+2 x^{1} y \frac{\partial}{\partial y}, \\
& x^{1} y \frac{\partial}{\partial x^{1}}-x^{1} x^{1} \frac{\partial}{\partial x^{2}}+y y \frac{\partial}{\partial y}
\end{aligned}
$$

one verifies that they all are tangent to the skeleton $y_{2}=\frac{1}{4}\left(y_{1}\right)^{2}, y_{1,1,1}=0$. Thus the bound is attained. One then verifies ([FK2005a]) that the spanned Lie algebra is isomorphic to $\mathfrak{s o}(5, \mathbb{C})$. 
Lemma 8.121. Assuming the normalizations of Lemma 8.54, the remainder $\mathrm{O}_{4}$ in (8.53) is an $\mathrm{O}_{3}\left(x^{1}, a^{1}\right)$ :

$$
y=b+\frac{2 x^{1} a^{1}+x^{1} x^{1} a^{2}+a^{1} a^{1} x^{2}}{1-x^{2} a^{2}}+\left(x^{1}\right)^{3} \mathrm{R}+\left(x^{1}\right)^{2} a^{1} \mathrm{R}+x^{1}\left(a^{1}\right)^{2} \mathrm{R}+\left(a^{1}\right)^{3} \mathrm{R}
$$

Proof. Indeed, writing

$$
y=b+x^{1} \Lambda^{1,0}+a^{1} \Lambda^{0,1}+x^{1} x^{1} \Lambda^{2,0}+x^{1} a^{1} \Lambda^{1,1}+a^{1} a^{1} \Lambda^{0,2}+\mathrm{O}_{3}\left(x^{1}, a^{1}\right),
$$

with $\Lambda^{i, j}=\Lambda^{i, j}\left(x^{2}, a^{2}\right)$, and developing the determinant (8.54) with respect to the powers of $\left(x^{1}, a^{1}\right)$, the vanishing of the coefficients of cst., of $x^{1}$, of $a^{1}$ yields the system

$$
\left\{\begin{array}{l}
0 \equiv \Lambda_{a^{2}}^{1,0} \Lambda_{x^{2}}^{0,1} \\
0 \equiv \Lambda^{1,1} \Lambda_{x^{2} a^{2}}^{1,0}-2 \Lambda_{a^{2}}^{2,0} \Lambda_{x^{2}}^{0,1}-\Lambda_{x^{2}}^{1,1} \Lambda_{a^{2}}^{1,0} \\
0 \equiv \Lambda^{1,1} \Lambda_{x^{2} a^{2}}^{0,1}-\Lambda_{a^{2}}^{1,1} \Lambda_{x^{2}}^{0,1}-2 \Lambda_{x^{2}}^{0,2} \Lambda_{a^{2}}^{1,0}
\end{array}\right.
$$

If the first equation yields $\Lambda_{a^{2}}^{1,0} \equiv 0$, replacing in the second, using $\Lambda^{2,0}=a^{2}+\mathrm{O}_{2}$, we deduce that $\Lambda_{x^{2}}^{0,1} \equiv 0$ also. Similarly, $\Lambda_{x^{2}}^{0,1} \equiv 0$ implies $\Lambda_{a^{2}}^{1,0} \equiv 0$. Since the coordinate system satisfies the normalization $\Pi(0, a) \equiv \Pi(x, 0) \equiv 0$, necessarily $\Lambda^{1,0}=\mathrm{O}\left(a^{2}\right)$ and $\Lambda^{0,1}=\mathrm{O}\left(x^{2}\right)$. We deduce:

$$
0 \equiv \Lambda^{1,0} \equiv \Lambda^{0,1} .
$$

Redeveloping the determinant, the vanishing of the coefficients of $x^{1} x^{1}$, of $x^{1} a^{1}$, of $a^{1} a^{1}$ yields the system

$$
\left\{\begin{array}{l}
0 \equiv \Lambda^{1,1} \Lambda_{x^{2} a^{2}}^{2,0}-2 \Lambda_{a^{2}}^{2,0} \Lambda_{x^{2}}^{1,1} \\
0 \equiv \Lambda^{1,1} \Lambda_{x^{2} a^{2}}^{1,1}-\Lambda_{a^{2}}^{1,1} \Lambda_{x^{2}}^{1,1}-4 \Lambda_{a^{2}}^{2,0} \Lambda_{x^{2}}^{0,2} \\
0 \equiv \Lambda^{1,1} \Lambda_{x^{2} a^{2}}^{0,2}-2 \Lambda_{x^{2}}^{0,2} \Lambda_{a^{2}}^{1,1}
\end{array}\right.
$$

Since $\Lambda^{1,1}(0)=2 \neq 0$, we may divide by $\Lambda^{1,1}$, obtaining a PDE system with the three functions $\Lambda_{x^{2} a^{2}}^{2,0}, \Lambda_{x^{2} a^{2}}^{1,1}, \Lambda_{x^{2} a^{2}}^{0,2}$ in the left hand side. We observe that the normalizations of Lemma 8.55 entail

$$
\Lambda^{2,0}=a^{2}+\mathrm{O}\left(x^{2} a^{2}\right)
$$$$
\Lambda^{1,1}=2+\mathrm{O}\left(x^{2} a^{2}\right),
$$$$
\Lambda^{0,2}=x^{2}+\mathrm{O}\left(x^{2} a^{2}\right) .
$$

By cross differentiations in the mentioned PDE system, it follows that all the Taylor coefficients of $\Lambda^{2,0}, \Lambda^{1,1}, \Lambda^{0,2}$ are uniquely determined. As already discovered in [GM2003b], the unique solution

$$
\Lambda^{2,0}=\frac{a^{2}}{1-x^{2} a^{2}}, \quad \Lambda^{1,1}=\frac{2}{1-x^{2} a^{2}}, \quad \Lambda^{0,2}=\frac{x^{2}}{1-x^{2} a^{2}},
$$

guarantees, when the remainder $\mathrm{O}_{3}\left(x^{1}, a^{1}\right)$ vanishes, that the determinant (8.45) indeed vanishes identically.

Conversely, suppose that $\operatorname{dim} \mathfrak{S} \mathfrak{Y} \mathfrak{M}\left(\mathcal{E}_{5}\right)=10$ is maximal.

With $\varepsilon \neq 0$ small, replacing $\left(x^{1}, x^{2}, y, a^{1}, a^{2}, b\right)$ by $\left(\varepsilon x^{1}, x^{2}, \varepsilon^{2} y, \varepsilon a^{1}, a^{2}, \varepsilon \varepsilon b\right)$ in (8.122) and dividing by $\varepsilon \varepsilon$, the remainder terms become small:

$$
y=b+\frac{2 x^{1} a^{1}+x^{1} x^{1} a^{2}+a^{1} a^{1} x^{2}}{1-x^{2} a^{2}}+\mathrm{O}(\varepsilon) .
$$

Then all the remainders in the equations $\Delta_{\mathcal{E}_{5}}$ of the skeleton are $\mathrm{O}(\varepsilon)$. We get ten generators similar to $(8.120)$, plus an $\mathrm{O}(\varepsilon)$ perturbation. Thanks to the rigidity of $\mathfrak{s o}(5, \mathbb{C})$, Theorem 5.15 provides a change of generators, close to the $10 \times 10$ identity matrix, leading to the same structure constants as those of the ten vector fields (8.120). As in the end 
of the proof of Theorem 5.13, we may then straighten some relevant vector fields (exercise) and finally check that their tangency to the skeleton implies that it is the model one. Theorem 8.117 is proved.

Corollary 8.130. Let $M \subset \mathbb{C}^{3}$ be a connected real analytic hypersurface whose Levi form has uniform rank 1 that is 2-nondegenerate at every point. Then

$$
\operatorname{dim} \mathfrak{h o r}(M) \leqslant 10,
$$

and the bound is attained if and only if $M$ is locally, in a neighborhood of Zariski-generic points, biholomorphic to the model $M_{0}$.

\section{$\S 9$. DUAL SYSTEM OF PARTIAL DIFFERENTIAL EQUATIONS}

9.1. Solvability with respect to the variables. Let $\mathcal{M}$ be as in $\S 2.10$ defined by $y=$ $\Pi(x, a, b)$ or dually by $b=\Pi^{*}(a, x, y)$.

Definition 9.2. $\mathcal{M}$ is solvable with respect to the variables if there exist an integer $\kappa^{*} \geqslant 1$ and multiindices $\delta(1), \ldots, \delta(n) \in \mathbb{N}^{p}$ with $|\delta(l)| \geqslant 1$ for $l=1, \ldots, n$ and $\max _{1 \leqslant l \leqslant n}|\delta(l)|=\kappa^{*}$, together with integers $j(1), \ldots, j(n)$ with $1 \leqslant j(l) \leqslant m$ such that the local $\mathbb{K}$-analytic map

$$
\mathbb{K}^{n+m} \ni(x, y) \longmapsto\left(\left(\Pi^{* j}(0, x, y)\right)^{1 \leqslant j \leqslant m},\left(\Pi_{a^{\delta(l)}}^{* j(l)}(0, x, y)\right)_{1 \leqslant l \leqslant n}\right) \in \mathbb{K}^{m+n}
$$

is of rank equal to $n+m$ at $(x, y)=(0,0)$

If $\mathcal{M}$ is a complexified generic submanifold, solvability with respect to the parameters is equivalent to solvability with respect to the variables, because $\Pi^{*}=\bar{\Pi}$. This is untrue in general: with $n=2, m=1$, consider the system $y_{x^{2}}=0, y_{x^{1} x^{1}}=0$, whose general solutions is $y(x)=b+x_{1} a$ with $x^{2}$ absent.

To characterize generally such a degeneration property, we develope both

$$
\begin{aligned}
y^{j} & =\Pi^{j}(x, a, b)=\sum_{\beta \in \mathbb{N}^{n}} x^{\beta} \Pi_{\beta}^{j}(a, b) \quad \text { and } \\
b^{j} & =\Pi^{* j}(a, x, y)=\sum_{\delta \in \mathbb{N}^{p}} a^{\delta} \Pi_{\delta}^{* j}(x, y),
\end{aligned}
$$

with analytic functions $\Pi_{\beta}^{j}(a, b), \Pi_{\delta}^{* j}(x, y)$ and we introduce two $\mathbb{K}^{\infty}$-valued maps

$$
\begin{array}{ll}
\mathcal{Q}_{\infty}: \quad(a, b) \longmapsto\left(\Pi_{\beta}^{j}(a, b)\right)_{\beta \in \mathbb{N}^{n}}^{1 \leqslant j \leqslant m} & \text { and } \\
\mathcal{Q}_{\infty}^{*}: \quad(x, y) \longmapsto\left(\Pi_{\delta}^{* j}(x, y)\right)_{\delta \in j \leqslant m}^{1 \leqslant j \leqslant m} .
\end{array}
$$

Since $b \mapsto\left(\Pi_{0}^{j}(0, b)\right)^{1 \leqslant j \leqslant m}$ and $y \mapsto\left(\Pi_{0}^{*}(0, y)\right)^{1 \leqslant j \leqslant m}$ are already both of rank $m$ at the origin, the generic ranks of these two maps, defined by testing the nonvanishing of minors of their infinite Jacobian matrices, satisfy

$$
\begin{aligned}
& \text { genrk } \mathcal{Q}_{\infty}=m+p_{\mathcal{M}} \quad \text { and } \\
& \text { genrk } \mathcal{Q}_{\infty}^{*}=m+n_{\mathcal{M}}
\end{aligned}
$$

for some two integers $0 \leqslant p_{\mathcal{M}} \leqslant p$ and $0 \leqslant n_{\mathcal{M}} \leqslant n$. So at a Zariski-generic point, the ranks are equal to $m+p_{\mathcal{M}}$ and to $m+n_{\mathcal{M}}$.

Proposition 9.7. There exists a local proper $\mathbb{K}$-analytic subset $\Sigma_{\mathcal{M}}$ of $\mathbb{K}_{x}^{n} \times \mathbb{K}_{y}^{m} \times \mathbb{K}_{a}^{p} \times \mathbb{K}_{b}^{m}$ whose equations, of the specific form

$$
\Sigma_{\mathcal{M}}=\left\{r_{\nu}(a, b)=0, \nu \in \mathbb{N}, \quad r_{\mu}^{*}(x, y)=0 \quad \mu \in \mathbb{N}\right\}
$$


are obtained by equating to zero all $\left(m+p_{\mathcal{M}}\right) \times\left(m+p_{\mathcal{M}}\right)$ minors of Jac $\mathcal{Q}_{\infty}$ and all $\left(m+n_{\mathcal{M}}\right) \times\left(m+n_{\mathcal{M}}\right)$ minors of Jac $\mathcal{Q}_{\infty}^{*}$, such that for every point $p=\left(x_{p}, y_{p}, a_{p}, b_{p}\right) \notin$ $\Sigma_{\mathcal{M}}$, there exists a local change of coordinates respecting the separation of the variables $(x, y)$ and $(a, b)$

$$
(x, y, a, b) \mapsto(\varphi(x, y), h(a, b))=:\left(x^{\prime}, y^{\prime}, a^{\prime}, b^{\prime}\right)
$$

by which $\mathcal{M}$ is transformed to a submanifold $\mathcal{M}^{\prime}$ centered and localized at $p^{\prime}=p$ having equations

$$
y^{\prime}=\Pi^{\prime}\left(x^{\prime}, a^{\prime}, b^{\prime}\right) \quad \text { and dually } \quad b^{\prime}=\Pi^{\prime *}\left(a^{\prime}, x^{\prime}, y^{\prime}\right)
$$

with $\Pi^{\prime}$ and $\Pi^{\prime *}$ independent of

$$
\left(x_{n_{\mathcal{M}}+1}^{\prime}, \ldots, x_{n}^{\prime}\right) \quad \text { and of } \quad\left(a_{p_{\mathcal{M}}+1}^{\prime}, \ldots, a_{p}^{\prime}\right) .
$$

So $\mathcal{M}^{\prime}$, may be considered to be living in $\mathbb{K}_{x^{\prime}}^{n_{\mathcal{M}}} \times \mathbb{K}_{y^{\prime}}^{m} \times \mathbb{K}_{a^{\prime}}^{p_{\mathcal{M}}} \times \mathbb{K}_{b^{\prime}}^{m}$ and in such a smaller space, at $p^{\prime}=p$, it is solvable both with respect to the parameters and to the variables.

Interpretation: by forgetting some innocuous variables, at a Zariski-generic point, any $\mathcal{M}$ is both solvable with respect to the parameters and to the variables. These two assumptions will be held up to the end of this Part I.

9.12. Dual system $\left(\mathcal{E}^{*}\right)$ and isomorphisms $\mathfrak{S Y M}(\mathcal{E}) \simeq \mathfrak{S} \mathfrak{Y} \mathfrak{M}\left(\mathcal{V}_{\mathcal{S}}(\mathcal{E})\right)=$ $\mathfrak{S Y M}\left(\mathcal{V}_{\mathcal{S}}\left(\mathcal{E}^{*}\right)\right) \simeq \mathfrak{S} \mathfrak{Y} \mathfrak{M}\left(\mathcal{E}^{*}\right)$. To a system $(\mathcal{E})$, we associate its submanifold of solutions $\mathcal{M}:=\mathcal{V}_{\mathcal{S}}(\mathcal{E})$. Assuming it to be solvable with respect to the variables and proceeding as in $\$ 2.10$, we can derive a dual system of completely integrable partial differential equations of the form

$$
b_{a^{\gamma}}^{j}(a)=G_{\gamma}^{j}\left(a, b(a),\left(b_{a^{\delta(l)}}^{j(l)}(a)\right)_{1 \leqslant l \leqslant n}\right),
$$

where $(j, \gamma) \neq(j, 0)$ and $\neq(j(l), \delta(l))$. Its submanifold of solutions $\mathcal{V}_{\mathcal{S}}\left(\mathcal{E}^{*}\right) \equiv \mathcal{V}_{\mathcal{S}}(\mathcal{E})$ has equations dual to those of $\mathcal{V}_{\mathcal{S}}(\mathcal{E})$.

Theorem 9.13. Under the assumption of twin solvability, we have:

$$
\mathfrak{S Y M}(\mathcal{E}) \simeq \mathfrak{S P M}\left(\mathcal{V}_{\mathcal{S}}(\mathcal{E})\right)=\mathfrak{S} \mathfrak{Y} \mathfrak{M}\left(\mathcal{V}_{\mathcal{S}}\left(\mathcal{E}^{*}\right)\right) \simeq \mathfrak{S Y M}\left(\mathcal{E}^{*}\right)
$$

through $\mathcal{L} \longleftrightarrow \mathcal{L}+\mathcal{L}^{*}=\mathcal{L}^{*}+\mathcal{L} \longleftrightarrow \mathcal{L}^{*}$.

\section{§10. FUNDAMENTAL PAIR OF FOLIATIONS AND COVERING PROPERTY}

10.1. Fundamental pair of foliations on $\mathcal{M}$. As in $\S 2$, let $(\mathcal{E})$ and $\mathcal{M}=\mathcal{V}_{\mathcal{S}}(\mathcal{E})$ be defined by $y=\Pi(x, a, b)$ or dually by $b=\Pi^{*}(a, x, y)$. Abbreviate

$$
z:=(x, y) \quad \text { and } \quad c:=(a, b) .
$$

Every transformation $(z, c) \mapsto(\varphi(z), h(c))$ belonging to $\mathrm{G}_{\mathrm{v}, \mathrm{p}}$ stabilizes both $\{z=$ cst. $\}$ and $\{c=$ cst. $\}$. Accordingly, the two foliations of $\mathcal{M}$

$$
\mathrm{F}_{\mathrm{v}}:=\bigcup_{c_{0}} \mathcal{M} \cap\left\{c=c_{0}\right\} \quad \text { and } \quad \mathrm{F}_{\mathrm{p}}:=\bigcup_{z_{0}} \mathcal{M} \cap\left\{z=z_{0}\right\}
$$

are invariant under changes of coordinates. We call $\left(F_{v}, F_{p}\right)$ the fundamental pair of foliations on $\mathcal{M}$. The leaves of the foliation by variables $\mathrm{F}_{\mathrm{v}}$ are $n$-dimensional:

$$
\mathrm{F}_{\mathrm{v}}\left(c_{0}\right)=\left\{\left(z, c_{0}\right): y=\Pi\left(x, c_{0}\right)\right\}
$$

The leaves of the foliation by parameters $\mathrm{F}_{\mathrm{p}}$ are $p$-dimensional:

$$
\mathrm{F}_{\mathrm{p}}\left(c_{0}\right)=\left\{\left(z_{0}, c\right): b=\Pi^{*}\left(a, z_{0}\right)\right\}
$$


We draw a diagram. In it, the positive codimension is invisible:

$$
m=\operatorname{dim} \mathcal{M}-\operatorname{dim} F_{v}-\operatorname{dim} F_{\mathrm{p}} \geqslant 1
$$

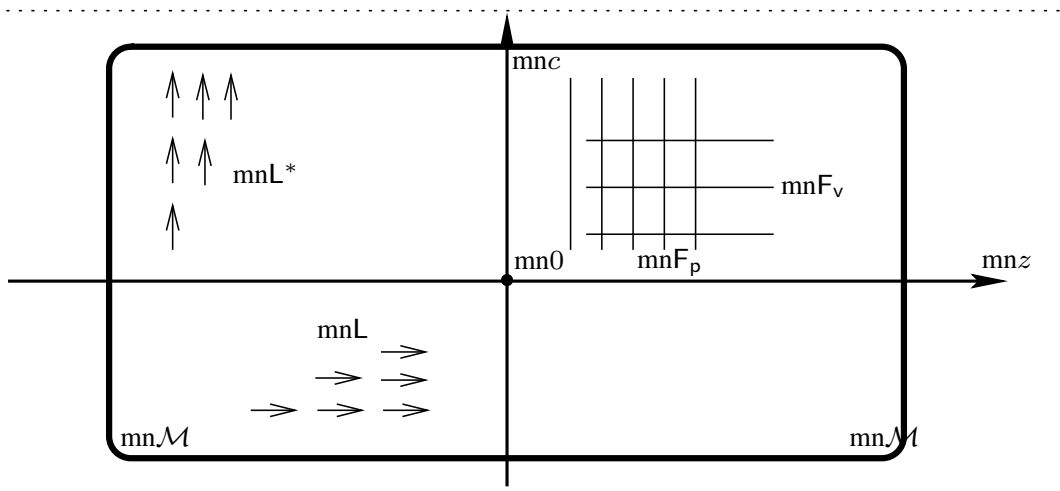

10.7. Chains $\Gamma_{k}$ and dual chains $\Gamma_{k}^{*}$. Similarly as in [GM2004, Me2005a, Me2005b, MP2005] (in a CR context), we introduce two collections $\left(\mathrm{L}_{k}\right)_{1 \leqslant k \leqslant n}$ and $\left(\mathrm{L}_{q}^{*}\right)_{1 \leqslant q \leqslant p}$ of vector fields whose integral manifolds coincide with the leaves of $F_{v}$ and of $F_{p}$ :

$$
\begin{cases}\mathrm{L}_{k}:=\frac{\partial}{\partial x_{k}}+\sum_{j=1}^{m} \frac{\partial \Pi^{j}}{\partial x_{k}}(x, a, b) \frac{\partial}{\partial y^{j}}, \quad k=1, \ldots, n, \\ \mathrm{~L}_{q}^{*}:=\frac{\partial}{\partial a^{q}}+\sum_{j=1}^{m} \frac{\partial \Pi^{* j}}{\partial a^{q}}(a, x, y) \frac{\partial}{\partial b^{j}}, \quad q=1, \ldots, p .\end{cases}
$$

Let $\left(z_{0}, c_{0}\right)=\left(x_{0}, y_{0}, a_{0}, b_{0}\right) \in \mathcal{M}$ be a fixed point, let $x_{1}:=\left(x_{1}^{1}, \ldots, x_{1}^{n}\right) \in \mathbb{K}^{n}$ and define the multiple flow map

$$
\left\{\begin{aligned}
\mathrm{L}_{x_{1}}\left(x_{0}, y_{0}, a_{0}, b_{0}\right) & :=\exp \left(x_{1} \mathrm{~L}\right)\left(p_{0}\right):=\exp \left(x_{1}^{n} \mathrm{~L}_{n}\left(\cdots\left(\exp \left(x_{1}^{1} \mathrm{~L}_{1}\left(z_{0}, c_{0}\right)\right)\right) \cdots\right)\right) \\
& :=\left(x_{0}+x_{1}, \Pi\left(x_{0}+x_{1}, a_{0}, b_{0}\right), a_{0}, b_{0}\right) .
\end{aligned}\right.
$$

Similarly, for $a_{1}=\left(a_{1}^{1}, \ldots, a_{1}^{p}\right) \in \mathbb{K}^{p}$, define the multiple flow map

$$
\mathrm{L}_{a_{1}}^{*}\left(x_{0}, y_{0}, a_{0}, b_{0}\right):=\left(x_{0}, y_{0}, a_{0}+a_{1}, \Pi^{*}\left(a_{0}+a_{1}, x_{0}, y_{0}\right)\right) .
$$

Starting from the $\left(z_{0}, c_{0}\right)=(0,0)$ and moving alternately along $\mathrm{F}_{\mathrm{v}}, \mathrm{F}_{\mathrm{p}}$, $\mathrm{F}_{\mathrm{v}}$, etc., we obtain

$$
\left\{\begin{aligned}
\Gamma_{1}\left(x_{1}\right) & :=\mathrm{L}_{x_{1}}(0), \\
\Gamma_{2}\left(x_{1}, a_{1}\right) & :=\mathrm{L}_{a_{1}}^{*}\left(\mathrm{~L}_{x_{1}}(0)\right), \\
\Gamma_{3}\left(x_{1}, a_{1}, x_{2}\right) & :=\mathrm{L}_{x_{2}}\left(\mathrm{~L}_{a_{1}}^{*}\left(\mathrm{~L}_{x_{1}}(0)\right)\right), \\
\Gamma_{4}\left(x_{1}, a_{1}, x_{2}, a_{2}\right) & :=\mathrm{L}_{a_{2}}^{*}\left(\mathrm{~L}_{x_{2}}\left(\mathrm{~L}_{a_{1}}^{*}\left(\mathrm{~L}_{x_{1}}(0)\right)\right)\right),
\end{aligned}\right.
$$

and so on. Generally, we get chains $\Gamma_{k}:=\Gamma_{k}\left([x a]_{k}\right)$, where $[x a]_{k}:=\left(x_{1}, a_{1}, x_{2}, a_{2}, \ldots\right)$ with exactly $k$ terms, where each $x_{l} \in \mathbb{K}^{n}$ and each $a_{l} \in \mathbb{K}^{p}$.

If, instead, the first movement consists in moving along $\mathrm{F}_{\mathrm{p}}$, we start with $\Gamma_{1}^{*}\left(a_{1}\right):=$ $\mathrm{L}_{a_{1}}^{*}(0), \Gamma_{2}^{*}\left(a_{1}, x_{1}\right):=\mathrm{L}_{x_{1}}\left(\mathrm{~L}_{a_{1}}^{*}(0)\right)$, etc., and generally we get dual chains $\Gamma_{k}^{*}\left([a x]_{k}\right)$, where $[a x]_{k}:=\left(a_{1}, x_{1}, a_{2}, x_{2}, \ldots\right)$, with exactly $k$ terms. Both $\Gamma_{k}$ and $\Gamma_{k}^{*}$ have range in $\mathcal{M}$.

For $k=1,2,3, \cdots$, integers $e_{k}$ and $e_{k}^{*}$ are defined inductively by

$$
\left\{\begin{array}{l}
e_{1}+e_{2}+e_{3}+\cdots+e_{k}=\operatorname{genrk}_{\mathbb{K}}\left(\Gamma_{k}\right), \\
e_{1}^{*}+e_{2}^{*}+e_{3}^{*}+\cdots+e_{k}^{*}=\operatorname{genrk}_{\mathbb{K}}\left(\Gamma_{k}^{*}\right) .
\end{array}\right.
$$

By (10.9) and (10.10), it is clear that $e_{1}=n, e_{2}=p, e_{1}^{*}=p$, and $e_{2}^{*}=n$. 
Example 10.13. For $y_{x x}=0$, the submanifold of solutions $\mathcal{M}$ is simply $y=b+x a$, whence

$$
\left\{\begin{aligned}
\Gamma_{1}\left(x_{1}\right) & =\left(x_{1}, 0,0,0\right) \\
\Gamma_{2}\left(x_{1}, a_{1}\right) & =\left(x_{1}, 0, a_{1},-x_{1} a_{1}\right) \\
\Gamma_{3}\left(x_{1}, a_{1}, x_{2}\right) & =\left(x_{1}+x_{2}, x_{2} a_{1}, a_{1},-x_{1} a_{1}\right) .
\end{aligned}\right.
$$

The rank at $(0,0,0)$ of $\Gamma_{3}$ is equal to two, not more. However, its generic rank is equal to three. Similar observations hold for the two submanifolds of solutions $y=b+x x a+x a a$ and $y=b+x a^{1}+x x a^{2}\left(\right.$ in $\left.\mathbb{K}^{5}\right)$.

Lemma 10.15. If genrk $_{\mathbb{K}}\left(\Gamma_{k+1}\right)=\operatorname{genrk}_{\mathbb{K}}\left(\Gamma_{k}\right)$, then for each positive integer $l \geqslant 1$, we have genrk $_{\mathbb{K}}\left(\Gamma_{k+l}\right)=\operatorname{genrk}_{\mathbb{K}}\left(\Gamma_{k}\right)$. The same stabilization property holds for $\Gamma_{k}^{*}$.

10.16. Covering property. We now formulate a central concept.

Definition 10.17. The pair of foliations $\left(F_{v}, F_{p}\right)$ is covering at the origin if there exists an integer $k$ such that the generic rank of $\Gamma_{k}$ is (maximal possible) equal to $\operatorname{dim}_{\mathbb{K}} \mathcal{M}$. Since for $a_{1}=0$, the dual $(k+1)$-th chain $\Gamma_{k+1}^{*}$ identifies with the $k$-th chain $\Gamma_{k}$, the same property holds for the dual chains.

Example 10.18. With $n=1, m=2$ and $p=1$ the submanifold defined by $y^{1}=b^{1}$ and $y^{2}=b^{2}+x a$ is twin solvable, but its pair of foliations is not covering at the origin. Then $\mathfrak{S P M}(\mathcal{M})$ is infinite-dimensional, since for $a=a\left(y^{1}\right)$ an arbitrary function, it contains $a\left(y^{1}\right) \frac{\partial}{\partial y^{1}}+a\left(b^{1}\right) \frac{\partial}{\partial b^{1}}$.

Because we aim only to study finite-dimensional Lie symmetry groups of partial differential equations, in the remainder of this Part I, we will constantly assume the covering property to hold.

By Lemma 10.15, there exist two well defined integers $\mu$ and $\mu^{*}$ such that $e_{3}, e_{4}, \ldots, e_{\mu+1}>0$, but $e_{\mu+l}=0$ for all $l \geqslant 2$ and similarly, $e_{3}^{*}, e_{4}^{*}, \ldots, e_{\mu^{*}+1}^{*}>0$, but $e_{\mu^{*}+l}^{*}=0$ for all $l \geqslant 2$. Since the pair of foliations is covering, we have the two dimension equalities

$$
\left\{\begin{aligned}
n+p+e_{3}+\cdots+e_{\mu+1} & =\operatorname{dim}_{\mathbb{K}} \mathcal{M}=n+m+p, \\
p+n+e_{3}^{*}+\cdots+e_{\mu^{*}+1}^{*} & =\operatorname{dim}_{\mathbb{K}} \mathcal{M}=n+m+p .
\end{aligned}\right.
$$

By definition, the ranges of $\Gamma_{\mu+1}$ and of $\Gamma_{\mu^{*}+1}^{*}$ cover (at least; more is true, see: Theorem 10.28) an open subset of $\mathcal{M}$. Also, it is elementary to verify the four inequalities

$$
\begin{array}{ll}
\mu \leqslant 1+m, & \mu^{*} \leqslant 1+m, \\
\mu \leqslant \mu^{*}+1, & \mu^{*} \leqslant \mu+1 .
\end{array}
$$

In fact, since $\Gamma_{k+1}$ with $x_{1}=0$ identifies with $\Gamma_{k}^{*}$, the second line follows.

Definition 10.21. The type of the covering pair of foliations $\left(F_{v}, F_{p}\right)$ is the pair of integers

$$
\left(\mu, \mu^{*}\right), \quad \text { with } \quad \max \left(\mu, \mu^{*}\right) \leqslant 1+m .
$$

Example 10.23. (Continued) We write down the explicit expressions of $\Gamma_{4}$ and of $\Gamma_{5}$ :

$$
\left\{\begin{aligned}
& \Gamma_{4}\left(x_{1}, a_{1}, x_{2}, a_{2} ; 0\right)=\left(x_{1}+x_{2}, x_{2} a_{1}, a_{1}+a_{2},-x_{1} a_{1}-x_{1} a_{2}-x_{2} a_{2},\right) \\
& \Gamma_{5}\left(x_{1}, a_{1}, x_{2}, a_{2}, x_{3} ; 0\right)=\left(x_{1}+x_{2}+x_{3}, x_{2} a_{1}+x_{3} a_{1}+x_{3} a_{2}, a_{1}+a_{2}\right. \\
&\left.-x_{1} a_{1}-x_{1} a_{2}-x_{2} a_{2}\right) .
\end{aligned}\right.
$$

Here, $\operatorname{dim} \mathcal{M}=3$. By computing its Jacobian matrix, $\Gamma_{5}$ is of rank 3 at every point $\left(x_{1}, a_{1}, 0,-a_{1},-x_{1}\right) \in \mathbb{K}^{5}$ with $a_{1} \neq 0$. Since (obviously)

$$
\Gamma_{5}\left(x_{1}, a_{1}, 0,-a_{1},-x_{1}\right)=0 \in \mathcal{M},
$$


we deduce that $\Gamma_{5}$ is submersive ("covering") from a small neighborhood of $\left(x_{1}, a_{1}, 0,-a_{1},-x_{1}\right)$ in $\mathbb{K}^{5}$ onto a neighborhood of the origin in $\mathcal{M}$.

10.26. Covering a neighborhood of the origin in $\mathcal{M}$. For $\left(z_{0}, c_{0}\right) \in \mathcal{M}$ fixed and close to the origin, we denote by $\Gamma_{k}\left([x a]_{k} ;\left(z_{0}, c_{0}\right)\right)$ and by $\Gamma_{k}^{*}\left([a x]_{k} ;\left(z_{0}, c_{0}\right)\right)$ the (dual) chains issued from $\left(z_{0}, c_{0}\right)$. For given parameters $[x a]_{k}=\left(x_{1}, a_{1}, x_{2}, \ldots\right)$, we denote by $[-x a]_{k}$ the collection $\left(\cdots,-x_{2},-a_{1},-x_{2}\right)$ with minus signs and reverse order; similarly, we introduce $[-a x]_{k}$. Notably, we have $\mathrm{L}_{-x_{1}}\left(\mathrm{~L}_{x_{1}}(0)\right)=0$ (because $\mathrm{L}_{-x_{1}+x_{1}}(\cdot)=\mathrm{L}_{0}(\cdot)=\mathrm{Id}$ ), and also $\mathrm{L}_{-x_{1}}\left(\mathrm{~L}_{-a_{1}}^{*}\left(\mathrm{~L}_{a_{1}}^{*}\left(\mathrm{~L}_{x_{1}}(0)\right)\right)\right)=0$ and generally:

$$
\Gamma_{k}\left([-x a]_{k} ; \Gamma_{k}\left([x a]_{k} ; 0\right)\right) \equiv 0 .
$$

Geometrically speaking, by following backward the $k$-th chain $\Gamma_{k}$, we come back to 0 .

Theorem 10.28. ([Me2005a, Me2005b], [*]) The two maps $\Gamma_{2 \mu+1}$ and $\Gamma_{2 \mu^{*}+1}^{*}$ are submersive onto a neighborhood of the origin in $\mathcal{M}$. Precisely, there exist two points $[x a]_{2 \mu+1}^{0} \in \mathbb{K}^{(\mu+1) n+\mu p}$ and $[a x]_{2 \mu^{*}+1}^{0} \in \mathbb{K}^{\mu^{*} n+\left(\mu^{*}+1\right) p}$ arbitrarily close to the origin with $\Gamma_{2 \mu+1}\left([x a]_{2 \mu+1}^{0}\right)=0$ and $\Gamma_{2 \mu^{*}+1}^{*}\left([a x]_{2 \mu^{*}+1}^{0}\right)=0$ such that the two maps

$$
\left\{\begin{array}{c}
\mathbb{K}^{(\mu+1) n+\mu p} \ni[x a]_{2 \mu+1} \longmapsto \Gamma_{2 \mu+1}\left([x a]_{2 \mu+1}\right) \in \mathcal{M} \quad \text { and } \\
\mathbb{K}^{\mu^{*} n+\left(\mu^{*}+1\right) p} \ni[a x]_{2 \mu^{*}+1} \longmapsto \Gamma_{2 \mu^{*}+1}^{*}\left([a x]_{2 \mu^{*}+1}\right) \in \mathcal{M}
\end{array}\right.
$$

are of rank $n+m+p=\operatorname{dim}_{\mathbb{K}} \mathcal{M}$ at the points $[x a]_{2 \mu}^{0}$ and $[a x]_{2 \mu^{*}}^{0}$ respectively. In particular, the ranges of the two maps $\Gamma_{2 \mu+1}$ and $\Gamma_{2 \mu^{*}+1}^{*}$ cover a neighborhood of the origin in $\mathcal{M}$.

Let $\pi_{z}(z, c):=z$ and $\pi_{c}(z, c):=c$ be the two canonical projections. The next corollary will be useful in Section 12. In the example above, it also follows that the map

$$
[x a]_{4} \mapsto \pi_{c}\left(\Gamma_{4}\left(\left[x a_{4}\right]\right)\right)=\left(a_{1}+a_{2},-x_{1} a_{1}-x_{1} a_{2}-x_{2} a_{2}\right) \in \mathbb{K}^{2}
$$

is of rank two at all points $[x a]_{4}^{0}:=\left(x_{1}^{0}, a_{1}^{0}, 0,-a_{1}^{0}\right)$ with $a_{1}^{0} \neq 0$.

Corollary 10.31. ([Me2005a, Me2005b], [*]) There exist two points $[x a]_{2 \mu}^{0} \in \mathbb{K}^{\mu(n+p)}$ and $[a x]_{2 \mu^{*}}^{0} \in \mathbb{K}^{\mu^{*}(n+p)}$ arbitrarily close to the origin with $\pi_{c}\left(\Gamma_{2 \mu}\left([x a]_{2 \mu}^{0}\right)\right)=0$ and $\pi_{z}\left(\Gamma_{2 \mu^{*}}^{*}\left([a x]_{2 \mu^{*}}^{0}\right)\right)=0$ such that the two maps

$$
\left\{\begin{array}{c}
\mathbb{K}^{\mu(n+p)} \ni[x a]_{2 \mu} \longmapsto \pi_{c}\left(\Gamma_{2 \mu}\left([x a]_{2 \mu}\right)\right) \in \mathbb{K}^{m+p} \quad \text { and } \\
\mathbb{K}^{\mu^{*}(n+p)} \ni[a x]_{2 \mu^{*}} \longmapsto \pi_{z}\left(\Gamma_{2 \mu^{*}}^{*}\left([a x]_{2 \mu^{*}}\right)\right) \in \mathbb{K}^{n+m}
\end{array}\right.
$$

are of rank $m+p$ at the point $[x a]_{2 \mu}^{0} \in \mathbb{K}^{\mu(n+p)}$ and of rank $n+m$ at the point $[a x]_{2 \mu^{*}}^{0} \in$ $\mathbb{K}^{\mu^{*}(n+p)}$.

In the case $m=1$ (single dependent variable $y \in \mathbb{K}$ ), the covering property always hold with $\mu=\mu^{*}=2$.

\section{$\S 11$. FORMAL AND SMOOTH EQUIVALENCES BETWEEN SUBMANIFOLDS OF SOLUTIONS}

11.1. Transformations of submanifolds of solutions. Lemma 7.3 shows that every equivalence $\varphi$ between two PDE systems $(\mathcal{E})$ and $\left(\mathcal{E}^{\prime}\right)$ lifts as a transformation which respects the separation between variables and parameters of the form

$$
(x, y, a, b) \longmapsto(\phi(x, y), \psi(x, y), f(a, b), g(a, b))=(\varphi(x, y), h(a, b))=:\left(x^{\prime}, y^{\prime}, a^{\prime}, b^{\prime}\right)
$$


from the source submanifolds of solutions $\mathcal{M}:=\mathcal{V}_{\mathcal{S}}(\mathcal{E})$ to the target $\mathcal{M}^{\prime}:=\mathcal{V}_{\mathcal{S}}\left(\mathcal{E}^{\prime}\right)$, whose equations are

$$
\begin{array}{cll}
y=\Pi(x, c) & \text { or dually } & b=\Pi^{*}(a, z) \quad \text { and } \\
y^{\prime}=\Pi^{\prime}\left(x^{\prime}, c^{\prime}\right) & \text { or dually } & b^{\prime}=\Pi^{\prime *}\left(a^{\prime}, z^{\prime}\right) .
\end{array}
$$

The study of transformations between submanifolds of solutions possesses strong similarities with the study of CR mappings between CR manifolds ([Pi1975, We1977, DW1980, BJT1985, DF1988, BER1999, Me2005a, Me2005b]). In fact, one may transfer the whole theory of the analytic reflection principle to this more general context. In the present $\S 10$ and in the next $\S 11$, we select and establish some of the results that are useful to the Lie theory. Some accessible open questions will also be formulated.

Maps of the form (11.2) send leaves of $F_{v}$ and of $F_{p}$ to leaves of $F_{v}^{\prime}$, and of $F_{p}^{\prime}$, respectively.

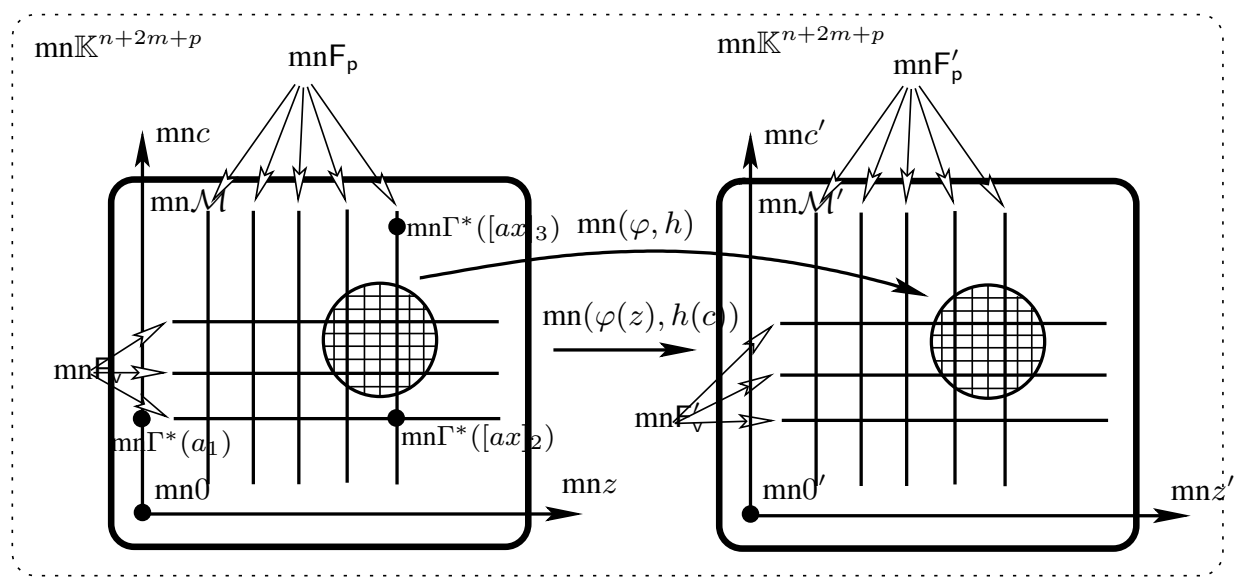

11.4. Regularity and jet parametrization. Some strong rigidity properties underly the above diagram. Especially, the smoothness of the two pairs $\left(F_{v}, F_{p}\right)$ and $\left(F_{v}^{\prime}, F_{p}^{\prime}\right)$ governs the smoothness of $(\varphi, h)$.

We shall study the regularity of a purely formal map $\left(z^{\prime}, c^{\prime}\right)=(\varphi(z), h(c))$, namely $\varphi(z) \in \mathbb{K} \llbracket z \rrbracket^{n+m}$ and $h(c) \in \mathbb{K} \llbracket c \rrbracket^{p+m}$, assuming $(\mathcal{E})$ and $\left(\mathcal{E}^{\prime}\right)$ to be analytic. Concretely, the assumption that $(\varphi, h)$ maps $\mathcal{M}$ to $\mathcal{M}^{\prime}$ reads as one of the four equivalent identities:

$$
\left\{\begin{aligned}
\psi(x, \Pi(x, c)) & \equiv \Pi^{\prime}(\phi(x, \Pi(x, c)), h(c)), \\
\psi(z) & \equiv \Pi^{\prime}\left(\phi(z), h\left(a, \Pi^{*}(a, z)\right),\right. \\
g\left(a, \Pi^{*}(a, z)\right) & \equiv \Pi^{\prime *}\left(f\left(a, \Pi^{*}(a, z)\right), \varphi(z)\right), \\
g(c) & \equiv \Pi^{\prime *}(f(c), \varphi(x, \Pi(x, c))),
\end{aligned}\right.
$$

in $\mathbb{K} \llbracket x, c \rrbracket^{m}$ and in $\mathbb{K} \llbracket a, z \rrbracket^{m}$.

Theorem 11.6. Let $(\varphi, h):=\mathcal{M} \rightarrow \mathcal{M}^{\prime}$ be a purely formal equivalence between two local $\mathbb{K}$-analytic submanifolds of solutions. Assume that the fundamental pair of foliations $\left(\mathrm{F}_{\mathrm{v}}, \mathrm{F}_{\mathrm{p}}\right)$ is covering at the origin, with type $\left(\mu, \mu^{*}\right)$ at the origin. Assume that $\mathcal{M}^{\prime}$ is both $\kappa$-solvable with respect to the parameters and $\kappa^{*}$-solvable with respect to the variables. Set $\ell:=\mu^{*}\left(\kappa+\kappa^{*}\right)$ and $\ell^{*}:=\mu\left(\kappa^{*}+\kappa\right)$. Then there exist two $\mathbb{K}^{n+m}$-valued and $\mathbb{K}^{p+m}$ valued local $\mathbb{K}$-analytic maps $\Phi_{\ell}$ and $H_{\ell^{*}}$, constructible only by means of $\Pi, \Pi^{*}, \Pi^{\prime}, \Pi^{\prime *}$, 
such that the following two formal power series identities hold:

$$
\left\{\begin{array}{l}
\varphi(z) \equiv \Phi_{\ell}\left(z, J_{z}^{\ell} \varphi(0)\right), \\
h(c) \equiv H_{\ell^{*}}\left(c, J_{c}^{\ell^{*}} h(0)\right),
\end{array}\right.
$$

in $\mathbb{K} \llbracket z \rrbracket^{n+m}$ and in $\mathbb{K} \llbracket c \rrbracket^{p+m}$, where $J_{z}^{\ell} \varphi(0)$ denotes the $\ell$-th jet of $h$ at the origin and similarly for $J_{c}^{\ell^{*}} h(0)$. In particular, as a corollary, we have the following two automatic regularity properties:

- $\varphi(z) \in \mathbb{K}\{z\}^{n+m}$ and $h(c) \in \mathbb{K}\{c\}^{p+m}$ are in fact convergent;

- if in addition $\mathcal{M}$ and $\mathcal{M}^{\prime}$ are $\mathbb{K}$-algebraic in the sense of Nash, then $\Phi_{\ell}$ and $H_{\ell^{*}}$ are also $\mathbb{K}$-algebraic, whence $\varphi(z) \in \mathcal{A}_{\mathbb{K}}\{z\}^{n+m}$ and $h(c) \in \mathcal{A}_{\mathbb{K}}\{c\}^{p+m}$ are in fact $\mathbb{K}$-algebraic.

Proof. We remind the explicit expressions of the two collections of vector fields spanning the leaves of the two foliations $F_{v}$ and $F_{p}$ :

$$
\begin{cases}\mathrm{L}_{k}:=\frac{\partial}{\partial x_{k}}+\sum_{j=1}^{m} \frac{\partial \Pi^{j}}{\partial x_{k}}(x, c) \frac{\partial}{\partial y^{j}}, \quad k=1, \ldots, n, \\ \mathrm{~L}_{q}^{*}:=\frac{\partial}{\partial a^{q}}+\sum_{j=1}^{m} \frac{\partial \Pi^{* j}}{\partial a^{q}}(a, z) \frac{\partial}{\partial b^{j}}, \quad q=1, \ldots, p .\end{cases}
$$

Observe that differentiating the first line of (11.5) with respect to $x^{k}$ amounts to applying the derivation $L_{k}$. Similarly, differentiating the third line of (11.5) with respect to $a^{q}$ amounts to applying $\mathrm{L}_{q}^{*}$. We thus get for $(z, c) \in \mathcal{M}$

$$
\left\{\begin{aligned}
\mathrm{L}_{k} \psi(z) & =\sum_{l=1}^{n} \frac{\partial \Pi^{\prime}}{\partial x^{\prime l}}(\phi(z), h(c)) \mathrm{L}_{k} \phi^{l}(z) \quad \text { and } \\
\mathrm{L}_{q}^{*} g(c) & =\sum_{r=1}^{p} \frac{\partial \Pi^{\prime *}}{\partial{a^{\prime}}^{r}}(f(c), \varphi(z)) \mathrm{L}_{q}^{*} f^{r}(c)
\end{aligned}\right.
$$

It follows from $\operatorname{det}\left(\frac{\partial \varphi^{k}}{\partial z^{l}}\right)(0) \neq 0$ and $\operatorname{det}\left(\frac{\partial h^{k}}{\partial c^{l}}\right)(0) \neq 0$ that the two formal determinants

$$
\operatorname{det}\left(\mathrm{L}_{k} \phi^{l}(z)\right)_{1 \leqslant k \leqslant n}^{1 \leqslant l \leqslant n} \quad \text { and } \quad \operatorname{det}\left(\mathrm{L}_{q}^{*} f^{r}(c)\right)_{1 \leqslant q \leqslant p}^{1 \leqslant r \leqslant p}
$$

have nonvanishing constant term. Consequently, these two matrices are invertible in $\mathbb{K} \llbracket z \rrbracket$ and in $\mathbb{K} \llbracket c \rrbracket$. So there exist universal polynomials $S_{l}^{j}$ and $S_{r}^{* j}$ such that

$$
\left\{\begin{array}{l}
\frac{\partial \Pi^{\prime j}}{\partial x^{\prime l}}(\varphi(z), h(c))=\frac{\mathrm{S}_{l}^{j}\left(\left\{\mathrm{~L}_{k^{\prime}} \varphi^{i^{\prime}}(z)\right\}_{1 \leqslant k^{\prime} \leqslant n}^{\substack{1 \leqslant i^{\prime} \leqslant n+m \\
1 \leqslant n}}\right)}{\operatorname{det}\left(\mathrm{L}_{k^{\prime}} \phi^{l^{\prime}}(z)\right)_{\substack{1 \leqslant l^{\prime} \leqslant n \\
1 \leqslant k^{\prime} \leqslant n}}^{1 \leqslant i^{\prime} \leqslant p+m}} \\
\frac{\partial \Pi^{\prime * j}}{\partial{a^{\prime \prime}}^{\prime r}}(f(c), \varphi(z))=\frac{\mathrm{S}_{r}^{* j}\left(\left\{\mathrm{~L}_{q^{\prime}}^{*} h^{i^{\prime}}(c)\right\}_{1 \leqslant q^{\prime} \leqslant p}\right)}{\operatorname{det}\left(\mathrm{L}_{q^{\prime}}^{*} f^{r^{\prime}}(c)\right)_{\substack{1 \leqslant r^{\prime} \leqslant p \\
1 \leqslant q^{\prime} \leqslant p}}}
\end{array}\right. \text { and }
$$

for $1 \leqslant j \leqslant m$, for $1 \leqslant l \leqslant n$, for $1 \leqslant r \leqslant p$ and for $(z, c) \in \mathcal{M}$. 
Again, we apply the vector fields $L_{k}$ to the obtained first line and the vector fields $L_{q}^{*}$ to the obtained second line, getting, thanks to the chain rule:

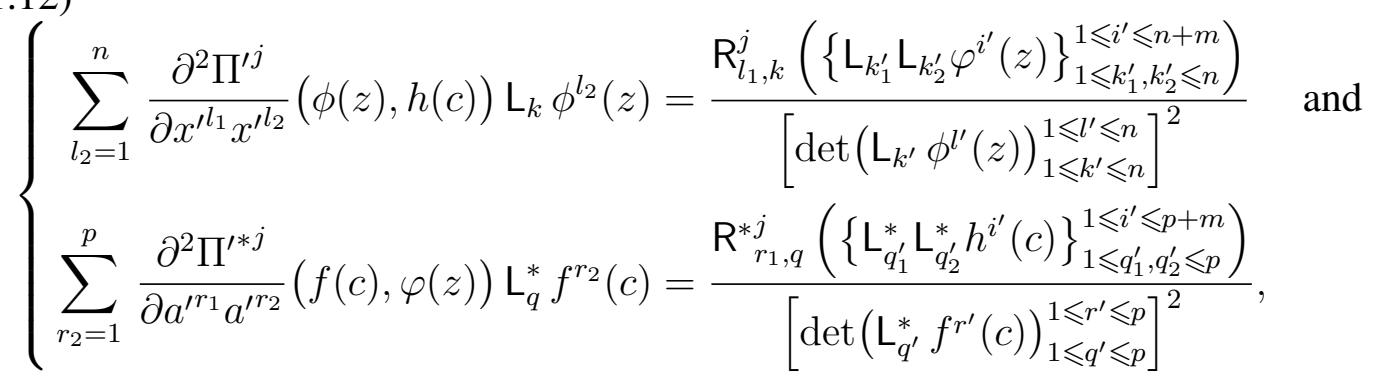

for $1 \leqslant j \leqslant m$, for $1 \leqslant l_{1}, l_{2} \leqslant n$, for $1 \leqslant r_{1}, r_{2} \leqslant p$ and for $(z, c) \in \mathcal{M}$. Here, $\mathrm{R}_{l_{1}, k}^{j}$ and $\mathrm{R}_{r_{1}, q}^{* j}$ are universal polynomials. Then applying once more Cramer's rule, we get

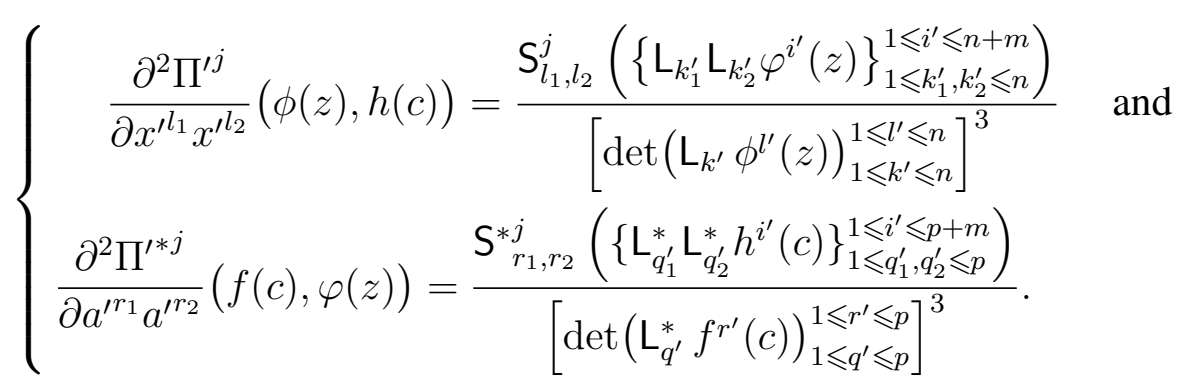

By induction, for every $j$ with $1 \leqslant j \leqslant m$ and every two multiindices $\beta \in \mathbb{N}^{n}$ and $\delta \in \mathbb{N}^{p}$, there exists two universal polynomials $S_{\beta}^{j}$ and $S_{\delta}^{* j}$ such that

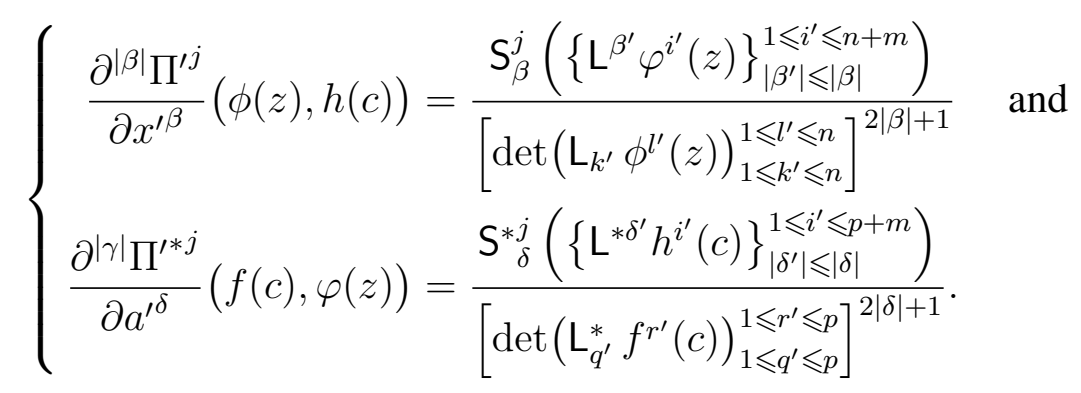

Here, for $\beta^{\prime} \in \mathbb{N}^{n}$, we denote by $\mathrm{L}^{\beta^{\prime}}$ the derivation of order $\left|\beta^{\prime}\right|$ defined by $\left(\mathrm{L}_{1}\right)^{\beta_{1}^{\prime}} \cdots\left(\mathrm{L}_{n}\right)^{\beta_{n}^{\prime}}$. Similarly, for $\delta^{\prime} \in \mathbb{N}^{p}, \mathrm{~L}^{* \delta^{\prime}}$ denotes the derivation of order $\left|\delta^{\prime}\right|$ defined by $\left(\mathrm{L}_{1}^{*}\right)^{\delta_{1}^{\prime}} \ldots\left(\mathrm{L}_{p}^{*}\right)^{\delta_{p}^{\prime}}$.

Next, by the assumption that $\mathcal{M}^{\prime}$ is solvable with respect to the parameters, there exist integers $j(1), \ldots, j(p)$ with $1 \leqslant j(q) \leqslant m$ and multiindices $\beta(1), \ldots, \beta(p) \in \mathbb{N}^{n}$ with $|\beta(q)| \geqslant 1$ and $\max _{1 \leqslant q \leqslant p}|\beta(q)|=\kappa$ such that the local $\mathbb{K}$-analytic map

$$
\mathbb{K}^{p+m} \ni c^{\prime} \longmapsto\left(\left(\Pi^{\prime j}\left(0, c^{\prime}\right)\right)^{1 \leqslant j \leqslant m},\left(\frac{\partial^{|\beta(q)|} \Pi^{\prime j(q)}}{\partial x^{\prime \beta(q)}}\left(0, c^{\prime}\right)\right)_{1 \leqslant q \leqslant p}\right) \in \mathbb{K}^{p+m}
$$

is of rank $p+m$ at $c^{\prime}=0$. Similarly, by the assumption that $\mathcal{M}^{\prime}$ is solvable with respect to the variables, there exist integers $j^{\sim}(1), \ldots, j^{\sim}(n)$ with $1 \leqslant j^{\sim}(l) \leqslant m$ and multiindices $\delta(1), \ldots, \delta(p) \in \mathbb{N}^{n}$ with $|\delta(q)| \geqslant 1$ and $\max _{1 \leqslant q \leqslant p}|\delta(q)|=\kappa^{*}$ such that the local $\mathbb{K}$ analytic map

$$
\mathbb{K}^{n+m} \ni z^{\prime} \longmapsto\left(\left(\Pi^{\prime * j}\left(0, z^{\prime}\right)\right)^{1 \leqslant j \leqslant m},\left(\frac{\partial^{|\delta(l)|} \Pi^{\prime * j^{\sim}(l)}}{\partial a^{\prime \delta(l)}}\left(0, z^{\prime}\right)\right)_{1 \leqslant l \leqslant n}\right) \in \mathbb{K}^{n+m}
$$


is of rank $n+m$ at $z^{\prime}=0$. We then consider from the first line of (11.14) only the $(p+m)$ equations written for $(j, 0),(j(q), \beta(q))$ and we solve $h(c)$ by means of the analytic implicit function theorem; also, in the second line of (11.14), we consider the $(n+m)$ equations written for $(j, 0),\left(j^{\sim}(l), \delta(l)\right)$ and we solve $\varphi(z)$. We get:

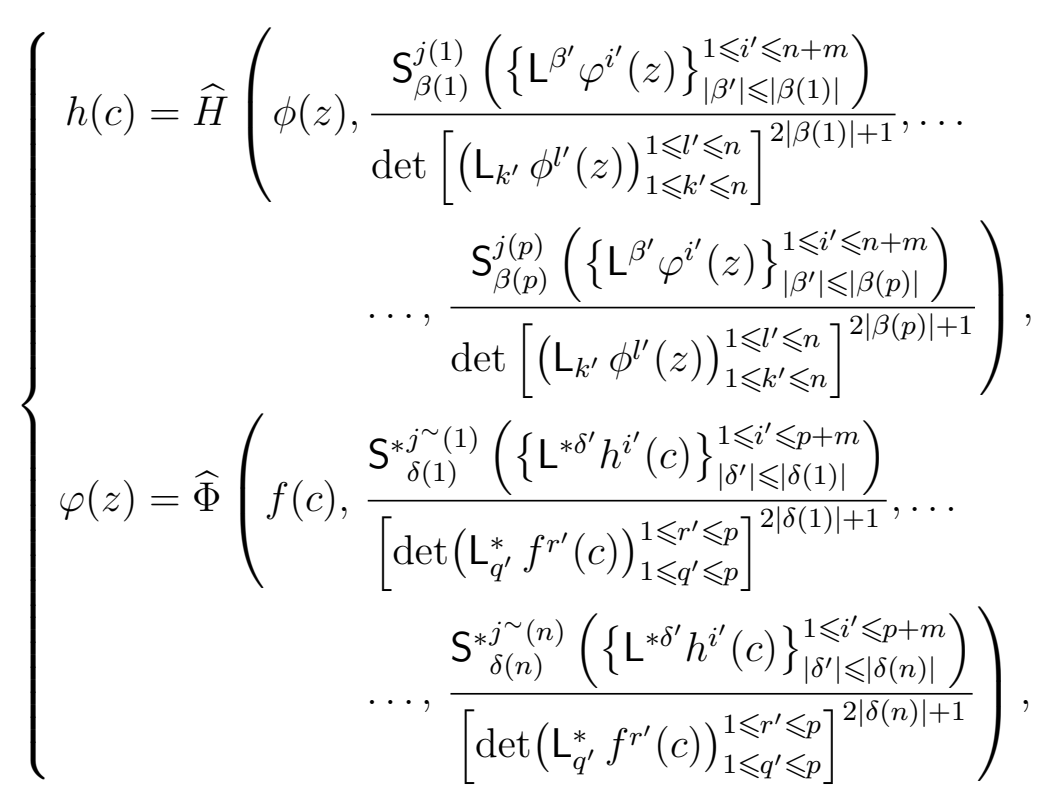

for $(z, c) \in \mathcal{M}$. The maps $\widehat{H}$ and $\widehat{\Phi}$ depend only on $\Pi^{\prime}, \Pi^{\prime *}$.

Lemma 11.18. For every $\beta^{\prime} \in \mathbb{N}^{n}$, there exists a universal polynomial $\mathrm{P}_{\beta^{\prime}}$ in the jet variables $J_{z}^{\left|\beta^{\prime}\right|}$ having $\mathbb{K}$-analytic coefficients in $(z, c)$ which depends only on $\Pi$, $\Pi^{*}$ such that, for $i^{\prime}=1, \ldots, n+m$ :

$$
\mathrm{L}^{\beta^{\prime}} \varphi^{i^{\prime}}(z) \equiv \mathrm{P}_{\beta^{\prime}}\left(z, c, J_{z}^{\left|\beta^{\prime}\right|} \varphi^{i^{\prime}}(z)\right)
$$

A similar property holds for $\mathrm{L}^{* \delta^{\prime}} h^{i^{\prime}}(c)$.

We deduce that there exist two local $\mathbb{K}$-analytic mappings $\Phi_{0}^{0}$ and $H_{0}^{0}$ such that we can write

$$
\left\{\begin{array}{l}
\varphi(z)=\Phi_{0}^{0}\left(z, c, J_{c}^{\kappa^{*}} h(c)\right), \\
h(c)=H_{0}^{0}\left(z, c, J_{z}^{\kappa} \varphi(z)\right),
\end{array}\right.
$$

for $(z, c) \in \mathcal{M}$. Concretely, this means that we have two equivalent pairs of formal identities

$$
\begin{aligned}
\varphi(z) & \equiv \Phi_{0}^{0}\left(z, a, \Pi^{*}(a, z), J_{c}^{\kappa^{*}} h\left(a, \Pi^{*}(a, z)\right)\right) \\
\varphi(x, \Pi(x, c)) & \equiv \Phi_{0}^{0}\left(x, \Pi(x, c), c, J_{c}^{\kappa^{*}} h(c)\right) \\
h(c) & \equiv H_{0}^{0}\left(x, \Pi(x, c), c, J_{z}^{\kappa} \varphi(x, \Pi(x, c))\right) \\
h\left(a, \Pi^{*}(a, z)\right) & \equiv H_{0}^{0}\left(z, a, \Pi^{*}(a, z), J_{z}^{\kappa} \varphi(z)\right)
\end{aligned}
$$

in $\mathbb{K} \llbracket a, z \rrbracket^{n+m}$ and in $\mathbb{K} \llbracket x, c \rrbracket^{p+m}$. We notice that, whereas $\varphi$ and $h$ are $a$ priori only purely formal, by construction, $\Phi_{0}^{0}$ and $H_{0}^{0}$ are $\mathbb{K}$-analytic near $\left(0,0, J_{c}^{\kappa^{*}} h(0)\right)$ and near $\left(0,0, J_{z}^{\kappa} \varphi(0)\right)$. 
Next, we introduce the following vector fields with $\mathbb{K}$-analytic coefficients tangent to $\mathcal{M}$ :

$$
\begin{cases}\mathrm{V}_{j}:=\frac{\partial}{\partial y^{j}}+\sum_{l=1}^{m} \frac{\partial \Pi^{* l}}{\partial y^{j}}(a, z) \frac{\partial}{\partial b^{l}}, \quad j=1, \ldots, m \quad \text { and } \\ \mathrm{V}_{j}^{*}:=\frac{\partial}{\partial b^{j}}+\sum_{l=1}^{m} \frac{\partial \Pi^{l}}{\partial b^{j}}(x, c) \frac{\partial}{\partial y^{l}}, \quad j=1, \ldots, m .\end{cases}
$$

Indeed, we check that $\mathrm{V}_{j_{1}}\left[b^{j_{2}}-\Pi^{* j_{2}}(a, z)\right] \equiv 0$ and that $\mathrm{V}_{j_{1}}^{*}\left[y^{j_{2}}-\Pi^{j_{2}}(x, c)\right] \equiv 0$.

For $\delta^{\prime} \in \mathbb{N}^{m}$, we observe that $\mathrm{V}^{\delta^{\prime}} \varphi=\frac{\partial^{\left|\delta^{\prime}\right|} \varphi}{\partial y^{\delta^{\prime}}}$. Applying then $\mathrm{L}^{\beta^{\prime}}$ with $\beta^{\prime} \in \mathbb{N}^{n}$, we get for $i=1, \ldots, n+m$ :

$$
\mathrm{L}^{\beta^{\prime}} \mathrm{V}^{\delta^{\prime}} \varphi^{i}(z)=\mathrm{Q}_{\beta^{\prime}, \delta^{\prime}}\left(z, c, J_{z}^{\left|\beta^{\prime}\right|+\left|\delta^{\prime}\right|} \varphi^{i}(z)\right)
$$

with $Q_{\beta^{\prime}, \delta^{\prime}}$ universal. Since the $n+m$ vector fields $\mathrm{L}_{k}$ and $\mathrm{V}_{j}$, having coefficients depending on $(z, c)$, span the tangent space to $\mathbb{K}_{x}^{n} \times \mathbb{K}_{y}^{m}$, the change of basis of derivations yields, by induction, the following.

Lemma 11.24. For every $\alpha \in \mathbb{N}^{n+m}$, there exists a universal polynomial $\mathrm{P}_{\alpha}$ in its last variables with coefficients being $\mathbb{K}$-analytic in $(z, c)$ and depending only on $\Pi, \Pi^{*}$ such that, for $i=1, \ldots, n+m$ :

$$
\partial_{z}^{\alpha} \varphi^{i}(z) \equiv \mathrm{P}_{\alpha}\left(z, c,\left(\mathrm{~L}^{\beta^{\prime}} \mathrm{V}^{\delta^{\prime}} \varphi^{i}(z)\right)_{\left|\beta^{\prime}\right|+\left|\delta^{\prime}\right| \leqslant|\alpha|}\right) .
$$

We are now in position to state and to prove the first fundamental technical lemma which generalizes the two formulas (11.20) to arbitrary jets.

Lemma 11.26. For every $\lambda \in \mathbb{N}$, there exist two local $\mathbb{K}$-analytic maps, $\Phi_{0}^{\lambda}$ valued in

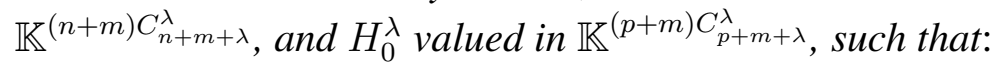

$$
\left\{\begin{aligned}
& J_{z}^{\lambda} \varphi(z) \equiv \Phi_{0}^{\lambda}\left(z, c, J_{c}^{\kappa^{*}+\lambda} h(c)\right), \\
& J_{c}^{\lambda} h(c) \equiv H_{0}^{\lambda}\left(z, c, J_{z}^{\kappa+\lambda} \varphi(z)\right) .
\end{aligned}\right.
$$

Proof. Consider for instance the first line. To obtain it, it suffices to apply the derivations $\mathrm{L}^{\beta^{\prime}} \mathrm{V}^{\delta^{\prime}}$ with $\left|\beta^{\prime}\right|+\left|\delta^{\prime}\right| \leqslant \lambda$ to the first line of (11.20), to use the chain rule and to apply Lemma 11.24.

Let $\theta \in \mathbb{K}^{l}, l \in \mathbb{N}$, let $Q(\theta)=\left(Q_{1}(\theta), \ldots, Q_{n+2 m+p}(\theta)\right) \in \mathbb{K} \llbracket \theta \rrbracket^{n+2 m+p}$ and let $a_{1} \in \mathbb{K}^{p}$. As the multiple flow of $\mathrm{L}^{*}$ given by (10.10) does not act on the variables $(x, y)$, we have the trivial but crucial property:

$$
\varphi\left(\mathrm{L}_{a_{1}}^{*}(Q(\theta))\right) \equiv \varphi\left(\pi_{z}\left(\mathrm{~L}_{a_{1}}^{*}(Q(\theta))\right)\right) \equiv \varphi\left(\pi_{z}(Q(\theta))\right) \equiv \varphi(Q(\theta)) .
$$

At the end, we allow to suppress the projection $\pi_{z}$ : this slight abuse of notation will lighten slightly the writting of further formulas. More generally, for $\lambda \in \mathbb{N}, a_{1} \in \mathbb{K}^{p}$, $x_{1} \in \mathbb{K}^{n}$ :

$$
\begin{aligned}
& J_{z}^{\lambda} \varphi\left(\mathrm{L}_{a_{1}}^{*}(Q(\theta))\right) \equiv J_{z}^{\lambda} \varphi(Q(\theta)) \quad \text { and } \\
& J_{c}^{\lambda} h\left(\mathrm{~L}_{x_{1}}(Q(\theta))\right) \equiv J_{c}^{\lambda} h(Q(\theta)) .
\end{aligned}
$$


As a consequence, for $2 k$ even and for $2 k+1$ odd, we have the following four cancellation relations, useful below (we drop $\pi_{z}$ and $\pi_{c}$ after $J_{z}^{\lambda} \varphi$ and after $J_{c}^{\lambda} h$ ):

$$
\left\{\begin{aligned}
J_{z}^{\lambda} \varphi\left(\Gamma_{2 k}\left([x a]_{2 k}\right)\right) & \equiv J_{z}^{\lambda} \varphi\left(\Gamma_{2 k-1}\left([x a]_{2 k-1}\right)\right), \\
J_{c}^{\lambda} h\left(\Gamma_{2 k}^{*}\left([a x]_{2 k}\right)\right) & \equiv J_{c}^{\lambda} h\left(\Gamma_{2 k-1}^{*}\left([a x]_{2 k-1}\right)\right), \\
J_{z}^{\lambda} \varphi\left(\Gamma_{2 k+1}^{*}\left([a x]_{2 k+1}\right)\right) & \equiv J_{z}^{\lambda} \varphi\left(\Gamma_{2 k}^{*}\left([a x]_{2 k}\right)\right), \\
J_{c}^{\lambda} h\left(\Gamma_{2 k+1}\left([x a]_{2 k+1}\right)\right) & \equiv J_{c}^{\lambda} h\left(\Gamma_{2 k}\left([x a]_{2 k}\right)\right) .
\end{aligned}\right.
$$

We are now in position to state and to prove the second main technical proposition.

Proposition 11.31. For every even chain-length $2 k$ and for every jet-height $\lambda$, there exist two local $\mathbb{K}$-analytic maps, $\Phi_{2 k}^{\lambda}$ valued in $\mathbb{K}^{(n+m) C_{n+m+\lambda}^{\lambda}}$, and $H_{2 k}^{\lambda}$ valued in $\mathbb{K}^{(p+m) C_{p+m+\lambda}^{\lambda}}$ such that:

$$
\left\{\begin{array}{l}
J_{z}^{\lambda} \varphi\left(\Gamma_{2 k}^{*}\left([a x]_{2 k}\right)\right) \equiv \Phi_{2 k}^{\lambda}\left([a x]_{2 k}, J_{z}^{k\left(\kappa+\kappa^{*}\right)+\lambda} \varphi(0)\right) \\
J_{c}^{\lambda} h\left(\Gamma_{2 k}\left([x a]_{2 k}\right)\right) \equiv H_{2 k}^{\lambda}\left([x a]_{2 k}, J_{c}^{k\left(\kappa+\kappa^{*}\right)+\lambda} \varphi(0)\right) .
\end{array}\right. \text { and }
$$

Similarly, for every odd chain length $2 k+1$ and for every jet eight $\lambda$, there exist two local $\mathbb{K}$-analytic maps, $\Phi_{2 k+1}^{\lambda}$ valued in $\mathbb{K}^{(n+m) C_{n+m+\lambda}^{\lambda}}$ and $H_{2 k+1}^{\lambda}$ valued in $\mathbb{K}^{(p+m) C_{p+m+\lambda}^{\lambda} \text {, }}$ such that:

$$
\left\{\begin{array}{l}
J_{z}^{\lambda} \varphi\left(\Gamma_{2 k+1}\left([x a]_{2 k+1}\right)\right) \equiv \Phi_{2 k+1}^{\lambda}\left([x a]_{2 k+1}, J_{c}^{k \kappa+(k+1) \kappa^{*}+\lambda} h(0)\right), \\
J_{c}^{\lambda} h\left(\Gamma_{2 k+1}^{*}\left([a x]_{2 k+1}\right)\right) \equiv H_{2 k+1}^{\lambda}\left([a x]_{2 k+1}, J_{z}^{(k+1) \kappa+k \kappa^{*}+\lambda} \varphi(0)\right) .
\end{array}\right.
$$

These maps depend only on $\Pi, \Pi^{*}, \Pi^{\prime}, \Pi^{\prime *}$.

Proof. For $2 k+1=1$, we replace $(z, c)$ by $\Gamma_{1}\left([x a]_{1}\right)$ in the first line of (11.27) and by $\Gamma_{1}^{*}\left([a x]_{1}\right)$ in the second line. Taking crucially account of the cancellation properties (11.29), we get:

$$
\left\{\begin{aligned}
J_{z}^{\lambda} \varphi\left(\Gamma_{1}\left([x a]_{1}\right)\right) & \equiv \Phi_{0}^{\lambda}\left(\Gamma_{1}\left([x a]_{1}\right), J_{c}^{\kappa^{*}+\lambda} h\left(\Gamma_{1}\left([x a]_{1}\right)\right)\right) \\
& \left.\equiv \Phi_{0}^{\lambda}\left(\Gamma_{1}\left([x a]_{1}\right), J_{c}^{\kappa^{*}+\lambda} h(0)\right)\right) \\
& =: \Phi_{1}^{\lambda}\left([x a]_{1}, J_{c}^{\kappa^{*}+\lambda} h(0)\right), \\
J_{c}^{\lambda} h\left(\Gamma_{1}^{*}\left([a x]_{1}\right)\right) & \equiv H_{0}^{\lambda}\left(\Gamma_{1}^{*}\left([a x]_{1}\right), J_{z}^{\kappa+\lambda} \varphi\left(\Gamma_{1}^{*}\left([a x]_{1}\right)\right)\right) \\
& \equiv H_{0}^{\lambda}\left(\Gamma_{1}^{*}\left([a x]_{1}\right), J_{z}^{\kappa+\lambda} \varphi(0)\right) \\
& =: H_{1}^{\lambda}\left([a x]_{1}, J_{z}^{\kappa+\lambda} \varphi(0)\right) .
\end{aligned}\right.
$$

Here, the third line defines $\Phi_{1}^{\lambda}$ and the sixth line defines $H_{1}^{\lambda}$. Thus, the proposition holds for $2 k+1=1$.

The rest of the proof proceeds by induction. We treat only the induction step from an odd chain-length $2 k+1$ to an even chain-length $2 k+2$, the other induction step being similar.

To this aim, we replace the variables $(z, c)$ in the first line of $(11.27)$ by $\Gamma_{2 k+2}^{*}\left([a x]_{2 k+2}\right)$. Taking account of the cancellation property and of the induction assumption:

$$
\begin{aligned}
J_{z}^{\lambda} \varphi\left(\Gamma_{2 k+2}^{*}\left([a x]_{2 k+2}\right)\right) & \equiv \Phi_{0}^{\lambda}\left(\Gamma_{2 k+2}^{*}\left([a x]_{2 k+2}\right), J_{c}^{\kappa^{*}+\lambda} h\left(\Gamma_{2 k+2}^{*}\left([a x]_{2 k+2}\right)\right)\right) \\
& \equiv \Phi_{0}^{\lambda}\left(\Gamma_{2 k+2}^{*}\left([a x]_{2 k+2}\right), J_{c}^{\kappa^{*}+\lambda} h\left(\Gamma_{2 k+1}^{*}\left([a x]_{2 k+1}\right)\right)\right) \\
& \equiv \Phi_{0}^{\lambda}\left(\Gamma_{2 k+2}^{*}\left([a x]_{2 k+2}\right), H_{2 k+1}^{\kappa^{*}+\lambda}\left([a x]_{2 k+1}, J_{c}^{(k+1)\left(\kappa+\kappa^{*}\right)+\lambda} \varphi(0)\right)\right) \\
& =: \Phi_{2 k+2}^{\lambda}\left([a x]_{2 k+2}, J_{c}^{(k+1)\left(\kappa+\kappa^{*}\right)+\lambda} \varphi(0)\right)
\end{aligned}
$$


The last line defines $\Phi_{2 k+2}^{\lambda}$. Similarly, we replace $(z, c)$ in the second line of (11.27) by $\Gamma_{2 k+2}\left([x a]_{2 k+2}\right)$. Taking account of the cancellation property and of the induction assumption:

(11.36)

$$
\begin{aligned}
J_{c}^{\lambda} h\left(\Gamma_{2 k+2}\left([x a]_{2 k+2}\right)\right) & \equiv H_{0}^{\lambda}\left(\Gamma_{2 k+2}\left([x a]_{2 k+2}\right), J_{c}^{\kappa+\lambda} \varphi\left(\Gamma_{2 k+2}\left([x a]_{2 k+2}\right)\right)\right) \\
& \equiv H_{0}^{\lambda}\left(\Gamma_{2 k+2}\left([x a]_{2 k+2}\right), J_{c}^{\kappa+\lambda} \varphi\left(\Gamma_{2 k+1}\left([x a]_{2 k+1}\right)\right)\right) \\
& \equiv H_{0}^{\lambda}\left(\Gamma_{2 k+2}\left([x a]_{2 k+2}\right), \Phi_{2 k+1}^{\kappa+\lambda}\left([x a]_{2 k+1}, J_{c}^{(k+1)\left(\kappa+\kappa^{*}\right)+\lambda} h(0)\right)\right) \\
& =: H_{2 k+2}^{\lambda}\left([x a]_{2 k+2}, J_{c}^{(k+1)\left(\kappa+\kappa^{*}\right)+\lambda} h(0)\right) .
\end{aligned}
$$

This completes the proof.

End of the proof of Theorem 11.6. With $\left(\mu, \mu^{*}\right)$ being the type of $\left(\mathrm{F}_{\mathrm{v}}, \mathrm{F}_{\mathrm{p}}\right)$ and with $[a x]_{2 \mu^{*}}^{0}$ given by Corollary 10.31, the rank property (10.32) insures the existence of an affine $(n+m)$-dimensional space $H \subset \mathbb{K}^{\mu^{*}(p+n)}$ passing through $[a x]_{2 \mu^{*}}^{0}$ and equipped with a local parametrization

$$
\mathbb{K}^{n+m} \ni s \mapsto[a x]_{2 \mu^{*}}(s) \in H
$$

satisfying $[a x]_{2 \mu^{*}}(0)=[a x]_{2 \mu^{*}}^{0}$, such that the map

$$
\mathbb{K}^{n+m} \ni s \longmapsto \pi_{z}\left(\Gamma_{2 \mu^{*}}^{*}\left([a x]_{2 \mu^{*}}(s)\right)\right)=: z(s) \in \mathbb{K}^{n+m}
$$

is a local diffeomorphism fixing $0 \in \mathbb{K}^{n+m}$. Replacing $z$ by $z(s)$ in $\varphi(z)$ and applying the formula in the first line of (11.32) with $\lambda=0$ and with $k=2 \mu^{*}$, we obtain

$$
\begin{aligned}
\varphi(z(s)) & =\varphi\left(\pi_{z}\left(\Gamma_{2 \mu^{*}}^{*}\left([a x]_{2 \mu^{*}}(s)\right)\right)\right. \\
& =\varphi\left(\Gamma_{2 \mu^{*}}^{*}\left([a x]_{2 \mu^{*}}(s)\right)\right) \\
& \equiv \Phi_{2 \mu^{*}}^{0}\left([a x]_{2 \mu^{*}}(s), J_{z}^{\mu^{*}\left(\kappa+\kappa^{*}\right)} \varphi(0)\right) .
\end{aligned}
$$

Inverting $s \mapsto z=z(s)$ as $z \mapsto s=s(z)$, we finally get

$$
\begin{aligned}
\varphi(z)=\varphi(z(s(z))) & \equiv \Phi_{2 \mu^{*}}^{0}\left([a x]_{2 \mu^{*}}(s(z)), J_{z}^{\mu^{*}\left(\kappa+\kappa^{*}\right)} \varphi(0)\right) \\
& =: \Phi_{\ell}\left(z, J_{z}^{\mu^{*}\left(\kappa+\kappa^{*}\right)} \varphi(0)\right),
\end{aligned}
$$

with $\ell:=\mu^{*}\left(\kappa+\kappa^{*}\right)$, where the last line defines $\Phi_{\ell}$. In conclusion, we have derived the first line of (11.7). The second one is obtained similarly.

If $\Pi, \Pi^{*}, \Pi^{\prime}, \Pi^{\prime *}$ are algebraic, so are $\Gamma_{k}, \Gamma_{k}^{*}, \widehat{H}, \widehat{\Phi}, \Phi_{0}^{\lambda}, H_{0}^{\lambda}, \Phi_{k}^{\lambda}, H_{k}^{\lambda}$ and $\Phi_{\ell}, H_{\ell^{*}}$.

The proof of Theorem 11.6 is complete. 


\section{II: Explicit prolongations of infinitesimal Lie symmetries}

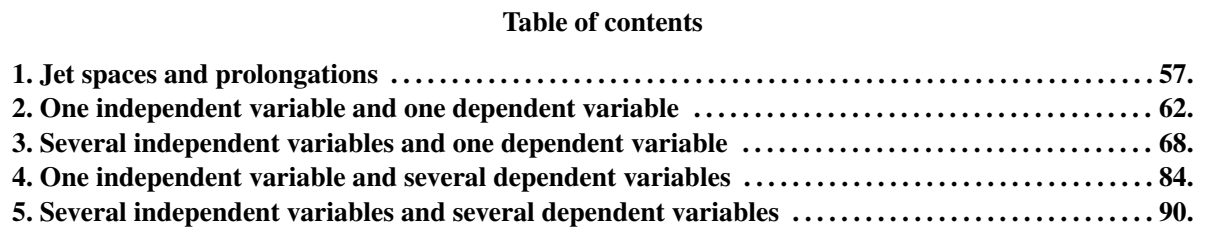

\section{$\S 1$. JET SPACES AND PROLONGATIONS}

1.1. Choice of notations for the jet space variables. Let $\mathbb{K}=\mathbb{R}$ or $\mathbb{C}$. Let $n \geqslant 1$ and $m \geqslant 1$ be two positive integers and consider two sets of variables $x=\left(x^{1}, \ldots, x^{n}\right) \in \mathbb{K}^{n}$ and $y=\left(y^{1}, \ldots, y^{m}\right)$. In the classical theory of Lie symmetries of partial differential equations, one considers certain differential systems whose (local) solutions should be mappings of the form $y=y(x)$. We refer to [O11986] and to [BK1989] for an exposition of the fundamentals of the theory. Accordingly, the variables $x$ are usually called independent, whereas the variables $y$ are called dependent. Not to enter in subtle regularity considerations (as in [Me2005b]), we shall assume $\mathcal{C}^{\infty}$-smoothness of all functions throughout this paper.

Let $\kappa \geqslant 1$ be a positive integer. For us, in a very concrete way (without fiber bundles), the $\kappa$-th jet space $\mathcal{J}_{n, m}^{\kappa}$ consists of the space $\mathbb{K}^{n+m+m \frac{(n+m) !}{n ! m !}}$ equipped with the affine coordinates

$$
\left(x^{i}, y^{j}, y_{i_{1}}^{j}, y_{i_{1}, i_{2}}^{j}, \ldots \ldots, y_{i_{1}, i_{2}, \ldots, i_{\kappa}}^{j}\right)
$$

having the symmetries

$$
y_{i_{1}, i_{2}, \ldots, i_{\lambda}}^{j}=y_{i_{\sigma(1)}, i_{\sigma(2)}, \ldots, i_{\sigma(\lambda)}}^{j},
$$

for every $\lambda$ with $1 \leqslant \lambda \leqslant \kappa$ and for every permutation $\sigma$ of the set $\{1, \ldots, \lambda\}$. The variable $y_{i_{1}, i_{2}, \ldots, i_{\lambda}}^{j}$ is an independent coordinate corresponding to the $\lambda$-th partial derivative

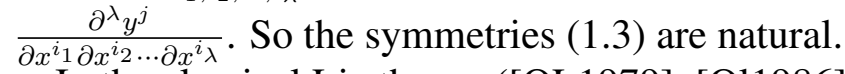

In the classical Lie theory ([OL1979], [O11986], [BK1989]), all the geometric objects: point transformations, vector fields, etc., are local, defined in a neighborhood of some point lying in some affine space $\mathbb{K}^{N}$. However, in this paper, the original geometric motivations are rapidly forgotten in order to focus on combinatorial considerations. Thus, to simplify the presentation, we shall not introduce any special notation to speak of certain local open subsets of $\mathbb{K}^{n+m}$, or of the jet space $\mathcal{J}_{n, m}^{\kappa}=\mathbb{K}^{n+m+m \frac{(n+m) !}{n ! m !}}$, etc.: we will always work in global affine spaces $\mathbb{K}^{N}$.

1.4. Prolongation $\varphi^{(\kappa)}$ of a local diffeomorphism $\varphi$ to the $\kappa$-th jet space. In this paragraph, we recall how the prolongation of a diffeomorphism to the $\kappa$-th jet space is defined ([OL1979], [O11986], [BK1989]).

Let $x_{*} \in \mathbb{K}^{n}$ be a central fixed point and let $\varphi: \mathbb{K}^{n+m} \rightarrow \mathbb{K}^{n+m}$ be a diffeomorphism whose Jacobian matrix is close to the identity matrix, at least in a small neighborhood of $x_{*}$. Let

$$
J_{x_{*}}^{\kappa}:=\left.\left(x_{*}^{i}, y_{* i_{1}}^{j}, y_{* i_{1}, i_{2}}^{j}, \ldots ., y_{* i_{1}, i_{2}, \ldots, i \kappa}^{j}\right) \in \mathcal{J}_{n, m}^{\kappa}\right|_{x_{*}}
$$

be an arbitrary $\kappa$-jet based at $x_{*}$. The goal is to defined its transformation $\varphi^{(\kappa)}\left(J_{x_{*}}^{\kappa}\right)$ by $\varphi$. 
To this aim, choose an arbitrary mapping $\mathbb{K}^{n} \ni x \mapsto g(x) \in \mathbb{K}^{m}$ defined at least in a neighborhood of $x_{*}$ and representing this $\kappa$-th jet, i.e. satisfying

$$
y_{* i_{1}, \ldots, i_{\lambda}}^{j}=\frac{\partial^{\lambda} g^{j}}{\partial x^{i_{1}} \cdots \partial x^{i_{\lambda}}}\left(x_{*}\right)
$$

for every $\lambda \in \mathbb{N}$ with $0 \leqslant \lambda \leqslant \kappa$, for all indices $i_{1}, \ldots, i_{\lambda}$ with $1 \leqslant i_{1}, \ldots, i_{\lambda} \leqslant n$ and for every $j \in \mathbb{N}$ with $1 \leqslant j \leqslant m$. In accordance with the splitting $(x, y) \in \mathbb{K}^{n} \times \mathbb{K}^{m}$ of coordinates, split the components of the diffeomorphism $\varphi$ as $\varphi=(\phi, \psi) \in \mathbb{K}^{n} \times \mathbb{K}^{m}$. Write $(\bar{x}, \bar{y})$ the coordinates in the target space, so that the diffeomorphism $\varphi$ is:

$$
\mathbb{K}^{n+m} \ni(x, y) \longmapsto(\bar{x}, \bar{y})=(\phi(x, y), \psi(x, y)) \in \mathbb{K}^{n+m} .
$$

Restrict the variables $(x, y)$ to belong to the graph of $g$, namely put $y:=g(x)$ above, which yields

$$
\left\{\begin{array}{l}
\bar{x}=\phi(x, g(x)) \\
\bar{y}=\psi(x, g(x))
\end{array}\right.
$$

As the differential of $\varphi$ at $x_{*}$ is close to the identity, the first family of $n$ scalar equations may be solved with respect to $x$, by means of the implicit function theorem. Denote $x=\bar{\chi}(\bar{x})$ the resulting mapping, satisfying by definition

$$
\bar{x} \equiv \phi(\bar{\chi}(\bar{x}), g(\bar{\chi}(\bar{x}))) .
$$

Replace $x$ by $\bar{\chi}(\bar{x})$ in the second family of $m$ scalar equations (1.8) above, which yields:

$$
\bar{y}=\psi(\bar{\chi}(\bar{x}), g(\bar{\chi}(\bar{x}))) .
$$

Denote simply by $\bar{y}=\bar{g}(\bar{x})$ this last relation, where $\bar{g}(\cdot):=\psi(\bar{\chi}(\cdot), g(\bar{\chi}(\cdot)))$.

In summary, the graph $y=g(x)$ has been transformed to the graph $\bar{y}=\bar{g}(\bar{x})$ by the diffeomorphism $\varphi$.

Define then the transformed jet $\varphi^{(\kappa)}\left(J_{x_{*}}^{\kappa}\right)$ to be the $\kappa$-th jet of $\bar{g}$ at the point $\bar{x}_{*}:=$ $\phi\left(x_{*}\right)$, namely:

$$
\varphi^{(\kappa)}\left(J_{x_{*}}^{\kappa}\right):=\left.\left(\frac{\partial^{\lambda} \bar{g}^{j}}{\partial \bar{x}^{i_{1}} \cdots \partial \bar{x}^{i_{\lambda}}}\left(\bar{x}_{*}\right)\right)_{1 \leqslant i_{1}, \ldots, i_{\lambda} \leqslant n, 0 \leqslant \lambda \leqslant \kappa}^{1 \leqslant j \leqslant m} \in \mathcal{J}_{n, m}^{\kappa}\right|_{\bar{x}_{*}} .
$$

It may be shown that this jet does not depend on the choice of a local graph $y=g(x)$ representing the $\kappa$-th jet $J_{x_{*}}^{\kappa}$ at $x_{*}$. Furthermore, if $\pi_{\kappa}:=\mathcal{J}_{n, m}^{\kappa} \rightarrow \mathbb{K}^{m}$ denotes the canonical projection onto the first factor, the following diagram commutes:

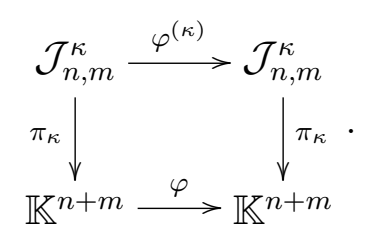

1.12. Inductive formulas for the $\kappa$-th prolongation $\varphi^{(\kappa)}$. To present them, we change our notations. Instead of $(\bar{x}, \bar{y})$, as coordinates in the target space $\mathbb{K}^{n} \times \mathbb{K}^{m}$, we shall use capital letters:

$$
\left(X^{1}, \ldots, X^{n}, Y^{1}, \ldots, Y^{m}\right) \text {. }
$$

In the source space $\mathbb{K}^{n+m}$ equipped with the coordinates $(x, y)$, we use the jet coordinates (1.2) on the associated $\kappa$-th jet space. In the target space $\mathbb{K}^{n+m}$ equipped with the coordinates $(X, Y)$, we use the coordinates

$$
\left(X^{i}, Y^{j}, Y_{X^{i_{1}}}^{j}, Y_{X^{i_{1}} X^{i_{2}}}^{j}, \ldots \ldots, Y_{X^{i_{1}} X^{i_{2} \ldots X^{i_{\kappa}}}}^{j}\right)
$$


on the associated $\kappa$-th jet space; to avoid confusion with $y_{i_{1}}, y_{i_{1}, i_{2}}, \ldots$ in subsequent formulas, we do not write $Y_{i_{1}}, Y_{i_{1}, i_{2}}, \ldots$ In these notations, the diffeomorphism $\varphi$ whose first order approximation is close to the identity mapping in a neighborhood of $x_{*}$ may be written under the form:

$$
\varphi:\left(x^{i^{\prime}}, y^{j^{\prime}}\right) \mapsto\left(X^{i}, Y^{j}\right)=\left(X^{i}\left(x^{i^{\prime}}, y^{j^{\prime}}\right), Y^{j}\left(x^{i^{\prime}}, y^{j^{\prime}}\right)\right)
$$

for some $\mathcal{C}^{\infty}$-smooth functions $X^{i}\left(x^{i^{\prime}}, y^{j^{\prime}}\right), i=1, \ldots, n$, and $Y^{j}\left(x^{i^{\prime}}, y^{j^{\prime}}\right), j=1, \ldots, m$. The first prolongation $\varphi^{(1)}$ of $\varphi$ may be written under the form:

$$
\varphi^{(1)}:\left(x^{i^{\prime}}, y^{j^{\prime}}, y_{i_{1}^{\prime}}^{j^{\prime}}\right) \longmapsto\left(X^{i}\left(x^{i^{\prime}}, y^{j^{\prime}}\right), Y^{j}\left(x^{i^{\prime}}, y^{j^{\prime}}\right), Y_{X^{i_{1}}}^{j}\left(x^{i^{\prime}}, y^{j^{\prime}}, y_{i_{1}^{\prime}}^{j^{\prime}}\right)\right),
$$

for some functions $Y_{X^{i_{1}}}^{j}\left(x^{i^{\prime}}, y^{j^{\prime}}, y_{i_{1}^{\prime}}^{j^{\prime}}\right)$ which depend on the pure first jet variables $y_{i_{1}^{\prime}}^{j^{\prime}}$. The way how these functions depend on the first order partial derivatives functions $X_{x^{i^{i}}}^{i}, X_{y^{j^{\prime}}}^{i}$, $Y_{x^{i^{\prime}}}^{j}, Y_{y^{j^{\prime}}}^{j}$ and on the pure first jet variables $y_{i_{1}^{\prime}}^{j^{\prime}}$ is provided (in principle) by the following compact formulas ([BK1989]):

$$
\left(\begin{array}{c}
Y_{X^{1}}^{j} \\
\vdots \\
Y_{X^{n}}^{j}
\end{array}\right)=\left(\begin{array}{ccc}
D_{1}^{1} X^{1} & \cdots & D_{1}^{1} X^{n} \\
\vdots & \cdots & \vdots \\
D_{n}^{1} X^{1} & \cdots & D_{n}^{1} X^{n}
\end{array}\right)^{-1}\left(\begin{array}{c}
D_{1}^{1} Y^{j} \\
\vdots \\
D_{n}^{1} Y^{j}
\end{array}\right)
$$

where, for $i^{\prime}=1, \ldots, n$, the symbol $D_{i^{\prime}}^{1}$ denotes the $i^{\prime}$-th first order total differentiation operator:

$$
D_{i^{\prime}}^{1}:=\frac{\partial}{\partial x^{i^{\prime}}}+\sum_{j^{\prime}=1}^{m} y_{i^{\prime}}^{j^{\prime}} \frac{\partial}{\partial y^{j^{\prime}}} .
$$

Striclty speaking, these formulas (1.17) are not explicit, because an inverse matrix is involved and because the terms $D_{i^{\prime}}^{1} X^{i}, D_{i^{\prime}}^{1} Y^{j}$ are not developed. However, it would be feasible and elementary to write down the corresponding totally explicit complete formulas for the functions $Y_{X^{i_{1}}}^{j}=Y_{X^{i_{1}}}^{j}\left(x^{i^{\prime}}, y^{j^{\prime}}, y_{i_{1}^{\prime}}^{j^{\prime}}\right)$.

Next, the second prolongation $\varphi^{(2)}$ is of the form

$$
\varphi^{(2)}:\left(x^{i^{\prime}}, y^{j^{\prime}}, y_{i_{1}^{\prime}}^{j^{\prime}}, y_{i_{1}^{\prime}, i_{2}^{\prime}}^{j^{\prime}}\right) \longmapsto\left(\varphi^{(1)}\left(x^{i^{\prime}}, y^{j^{\prime}}, y_{i_{1}^{\prime}}^{j^{\prime}}\right), Y_{X^{i_{1}} X^{i_{2}}}^{j}\left(x^{i^{\prime}}, y^{j^{\prime}}, y_{i_{1}^{\prime}}^{j^{\prime}}, y_{i_{1}^{\prime}, i_{2}^{\prime}}^{j^{\prime}}\right)\right),
$$

for some functions $Y_{X^{i_{1}} X^{i_{2}}}^{j}\left(x^{i^{\prime}}, y^{j^{\prime}}, y_{i_{1}^{\prime}}^{j^{\prime}}, y_{i_{1}^{\prime}, i_{2}^{\prime}}^{j^{\prime}}\right)$ which depend on the pure first and second jet variables. For $i=1, \ldots, n$, the expressions of $Y_{X^{i_{1} X^{i}}}^{j}$ are given by the following compact formulas (again [BK1989]):

$$
\left(\begin{array}{c}
Y_{X^{i_{1} X^{1}}}^{j} \\
\vdots \\
Y_{X^{i_{1} X^{n}}}^{j}
\end{array}\right)=\left(\begin{array}{ccc}
D_{1}^{1} X^{1} & \cdots & D_{1}^{1} X^{n} \\
\vdots & \cdots & \vdots \\
D_{n}^{1} X^{1} & \cdots & D_{n}^{1} X^{n}
\end{array}\right)^{-1}\left(\begin{array}{c}
D_{1}^{2} Y_{X^{i_{1}}}^{j} \\
\vdots \\
D_{n}^{2} Y_{X^{i_{1}}}^{j}
\end{array}\right)
$$

where, for $i^{\prime}=1, \ldots, n$, the symbol $D_{i^{\prime}}^{2}$ denotes the $i^{\prime}$-th second order total differentiation operator:

$$
D_{i^{\prime}}^{2}:=\frac{\partial}{\partial x^{i^{\prime}}}+\sum_{j^{\prime}=1}^{m} y_{i^{\prime}}^{j^{\prime}} \frac{\partial}{\partial y^{j^{\prime}}}+\sum_{j^{\prime}=1}^{m} \sum_{i_{1}^{\prime}=1}^{n} y_{i^{\prime}, i_{1}^{\prime}}^{j^{\prime}} \frac{\partial}{\partial y_{i_{1}^{\prime}}^{j^{\prime}}} .
$$

Again, these formulas (1.20) are not explicit in the sense that an inverse matrix is involved and that the terms $D_{i^{\prime}}^{1} X^{i}, D_{i^{\prime}}^{2} Y_{X^{i_{1}}}^{j}$ are not developed. It would already be a nontrivial 
computational task to develope these expressions and to find out some nice satisfying combinatorial formulas.

In order to present the general inductive non-explicit formulas for the computation of the $\kappa$-th prolongation $\varphi^{(\kappa)}$, we need some more notation. Let $\lambda \in \mathbb{N}$ be an arbitrary integer. For $i^{\prime}=1, \ldots, n$, let $D_{i^{\prime}}^{\lambda}$ denotes the $i^{\prime}$-th $\lambda$-th order total differentiation operators, defined precisely by:

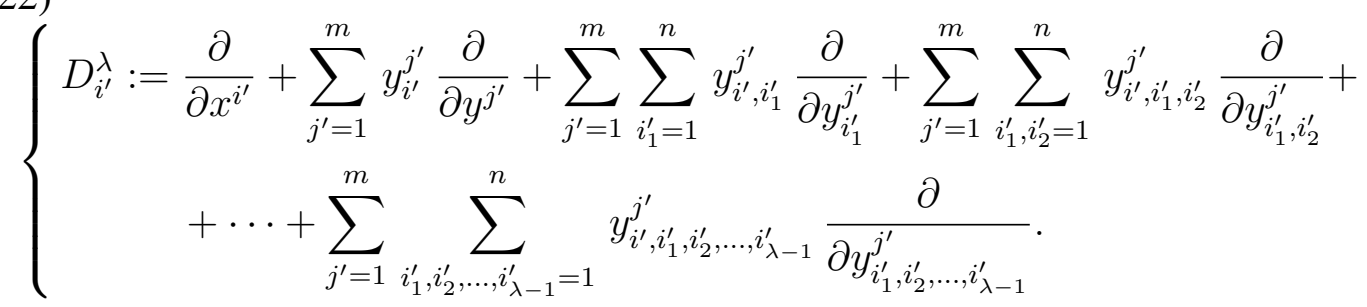

Then, for $i=1, \ldots, n$, the expressions of $Y_{X^{i_{1} \ldots X^{i} \lambda-1} X^{i}}^{j}$ are given by the following compact formulas (again [BK1989]):

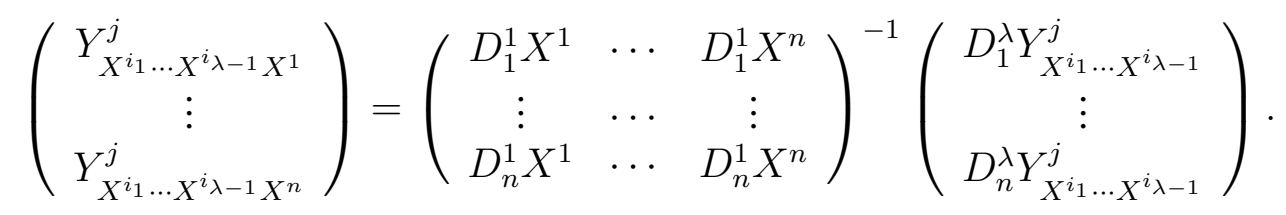

Again, these inductive formulas are incomplete and unsatisfactory.

Problem 1.24. Find totally explicit complete formulas for the $\kappa$-th prolongation $\varphi^{(\kappa)}$.

Except in the cases $\kappa=1,2$, we have not been able to solve this problem. The case $\kappa=1$ is elementary. Complete formulas in the particular cases $\kappa=2, n=1, m \geqslant 1$ and $n \geqslant 1, m=1$ are implicitely provided in [Me2004] and in Section ?(?), where one observes the appearance of some modifications of the Jacobian determinant of the diffeomorphism $\varphi$, inserted in a clearly understandable combinatorics. In fact, there is a nice dictionary between the formulas for $\varphi^{(2)}$ and the formulas for the second prolongation $\mathcal{L}^{(2)}$ of a vector field $\mathcal{L}$ which were written in equation (43) of [GM2003a] (see also equations (2.6), (3.20), (4.6) and (5.3) in the next paragraphs). In the passage from $\varphi^{(2)}$ to $\mathcal{L}^{(2)}$, a sort of formal first order linearization may be observed and the reverse passage may be easily guessed. However, for $\kappa \geqslant 3$, the formulas for $\varphi^{(\kappa)}$ explode faster than the formulas for the $\kappa$-th prolongation $\mathcal{L}^{(\kappa)}$ of a vector field $\mathcal{L}$. Also, the dictionary between $\varphi^{(\kappa)}$ and $\mathcal{L}^{(\kappa)}$ disappears. In fact, to elaborate an appropriate dictionary, we believe that one should introduce before a sort of formal $(\kappa-1)$-th order linearizations of $\varphi^{(\kappa)}$, finer than the first order linearization $\mathcal{L}^{(\kappa)}$. To be optimistic, we believe that the final answer to Problem 1.24 is, nevertheless, accessible after hard work.

The present article is devoted to present totally explicit complete formulas for the $\kappa$-th prolongation $\mathcal{L}^{(\kappa)}$ of a vector field $\mathcal{L}$ to $\mathcal{J}_{n, m}^{\kappa}$, for $n \geqslant 1$ arbitrary, for $m \geqslant 1$ arbitrary and for $\kappa \geqslant 1$ arbitrary.

1.25. Prolongation of a vector field to the $\kappa$-th jet space. Consider a vector field

$$
\mathcal{L}=\sum_{i=1}^{n} \mathcal{X}^{i}(x, y) \frac{\partial}{\partial x^{i}}+\sum_{j=1}^{m} \mathcal{Y}^{j}(x, y) \frac{\partial}{\partial y^{j}},
$$

defined in $\mathbb{K}^{n+m}$. Its flow:

$$
\varphi_{t}(x, y):=\exp (t \mathcal{L})(x, y)
$$

constitutes a one-parameter family of diffeomorphisms of $\mathbb{K}^{n+m}$ close to the identity. The lift $\left(\varphi_{t}\right)^{(\kappa)}$ to the $\kappa$-th jet space constitutes a one-parameter family of diffeomorphisms of 
$\mathcal{J}_{n, m}^{\kappa}$. By definition, the $\kappa$-th prolongation $\mathcal{L}^{(\kappa)}$ of $\mathcal{L}$ to the jet space $\mathcal{J}_{n, m}^{\kappa}$ is the infinitesimal generator of $\left(\varphi_{t}\right)^{(\kappa)}$, namely:

$$
\mathcal{L}^{(\kappa)}:=\left.\frac{d}{d t}\right|_{t=0}\left[\left(\varphi_{t}\right)^{(\kappa)}\right] .
$$

1.29. Inductive formulas for the $\kappa$-th prolongation $\mathcal{L}^{(\kappa)}$. As a vector field defined in $\mathbb{K}^{n+m+m \frac{(n+m) !}{n ! m !}}$, the $\kappa$-th prolongation $\mathcal{L}^{(\kappa)}$ may be written under the general form:

$$
\left\{\begin{aligned}
\mathcal{L}^{(\kappa)}= & \sum_{i=1}^{n} \mathcal{X}^{i} \frac{\partial}{\partial x^{i}}+\sum_{j=1}^{m} \mathcal{Y}^{j} \frac{\partial}{\partial y^{j}}+ \\
& +\sum_{j=1}^{m} \sum_{i_{1}=1}^{n} \mathbf{Y}_{i_{1}}^{j} \frac{\partial}{\partial y_{i_{1}}^{j}}+\sum_{j=1}^{m} \sum_{i_{1}, i_{2}=1}^{n} \mathbf{Y}_{i_{1}, i_{2}}^{j} \frac{\partial}{\partial y_{i_{1}, i_{2}}^{j}}+\cdots+ \\
& +\sum_{j=1}^{m} \sum_{i_{1}, \ldots, i_{\kappa}=1}^{n} \mathbf{Y}_{i_{1}, \ldots, i_{\kappa}}^{j} \frac{\partial}{\partial y_{i_{1}, \ldots, i_{\kappa}}^{j}} .
\end{aligned}\right.
$$

Here, the coefficients $\mathbf{Y}_{i_{1}}^{j}, \mathbf{Y}_{i_{1}, i_{2}}^{j}, \ldots, \mathbf{Y}_{i_{1}, i_{2}, \ldots, i_{\kappa}}^{j}$ are uniquely determined in terms of partial derivatives of the coefficients $\mathcal{X}^{i}$ and $\mathcal{Y}^{j}$ of the original vector field $\mathcal{L}$, together with the pure jet variables $\left(y_{i_{1}}^{j}, \ldots, y_{i_{1}, \ldots, i_{\kappa}}^{j}\right)$, by means of the following fundamental inductive formulas ([OL1979], [O11986], [BK1989]):

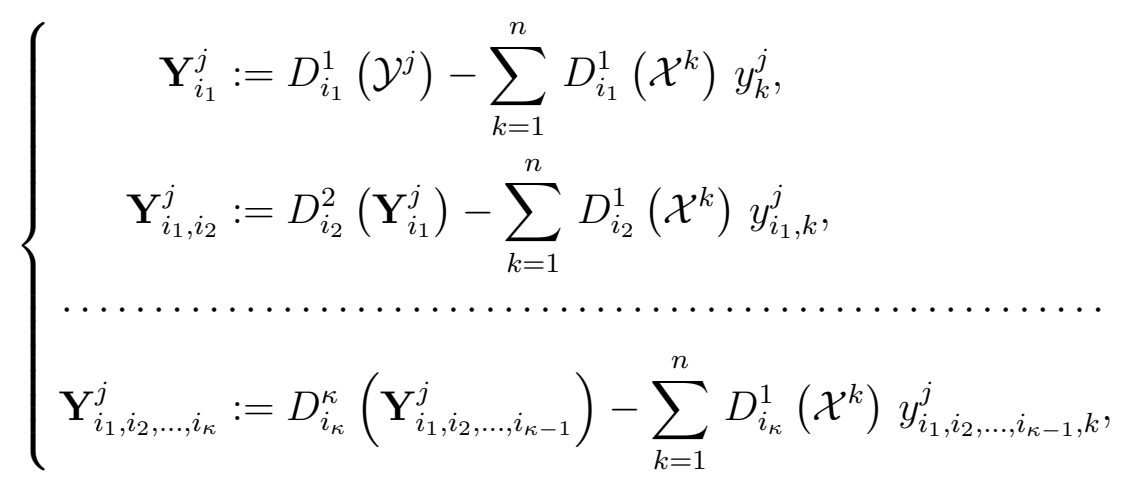

where, for every $\lambda \in \mathbb{N}$ with $0 \leqslant \lambda \leqslant \kappa$, and for every $i \in \mathbb{N}$ with $1 \leqslant i^{\prime} \leqslant n$, the $i^{\prime}$-th $\lambda$-th order total differentiation operator $D_{i^{\prime}}^{\lambda}$ was defined in (1.22) above.

Problem 1.32. Applying these inductive formulas, find totally explicit complete formulas for the $\kappa$-th prolongation $\mathcal{L}^{(\kappa)}$.

The present article is devoted to provide all the desired formulas.

1.33. Methodology of induction. We have the intention of presenting our results in a purely inductive style, based on several thorough visual comparisons between massive formulas which will be written and commented in four different cases:

(i) $n=1$ and $m=1 ; \kappa \geqslant 1$ arbitrary;

(ii) $n \geqslant 1$ and $m=1 ; \kappa \geqslant 1$ arbitrary;

(iii) $n=1$ and $m \geqslant 1 ; \kappa \geqslant 1$ arbitrary;

(iv) general case: $n \geqslant 1$ and $m \geqslant 1 ; \kappa \geqslant 1$ arbitrary.

Accordingly, we shall particularize and slightly lighten our notations in each of the three (preliminary) cases (i) [Section 2], (ii) [Section 3] and (iii) [Section 4]. 


\section{§2. ONE INDEPENDENT VARIABLE AND ONE DEPENDENT VARIABLE}

2.1. Simplified adapted notations. Assume $n=1$ and $m=1$, let $\kappa \in \mathbb{N}$ with $\kappa \geqslant 1$ and simply denote the jet variables by:

$$
\left(x, y, y_{1}, y_{2}, \ldots, y_{\kappa}\right) \in \mathcal{J}_{1,1}^{\kappa} .
$$

The $\kappa$-th prolongation of a vector field $\mathcal{L}=\mathcal{X} \frac{\partial}{\partial x}+\mathcal{Y} \frac{\partial}{\partial y}$ will be denoted by:

$$
\mathcal{L}^{(\kappa)}=\mathcal{X} \frac{\partial}{\partial x}+\mathcal{Y} \frac{\partial}{\partial y}+\mathbf{Y}_{1} \frac{\partial}{\partial y_{1}}+\mathbf{Y}_{2} \frac{\partial}{\partial y_{2}}+\cdots+\mathbf{Y}_{\kappa} \frac{\partial}{\partial y_{\kappa}}
$$

The coefficients $\mathbf{Y}_{1}, \mathbf{Y}_{2}, \ldots, \mathbf{Y}_{\kappa}$ are computed by means of the inductive formulas:

$$
\left\{\begin{array}{l}
\mathbf{Y}_{1}:=D^{1}(\mathcal{Y})-D^{1}(\mathcal{X}) y_{1} \\
\mathbf{Y}_{2}:=D^{2}\left(\mathbf{Y}_{1}\right)-D^{1}(\mathcal{X}) y_{2} \\
\ldots \ldots \ldots \ldots \ldots \ldots \ldots \ldots \ldots \ldots \ldots \\
\mathbf{Y}_{\kappa}:=D^{\kappa}\left(\mathbf{Y}_{\kappa-1}\right)-D^{1}(\mathcal{X}) y_{\kappa}
\end{array}\right.
$$

where, for $1 \leqslant \lambda \leqslant \kappa$ :

$$
D^{\lambda}:=\frac{\partial}{\partial x}+y_{1} \frac{\partial}{\partial y}+y_{2} \frac{\partial}{\partial y_{1}}+\cdots+y_{\lambda} \frac{\partial}{\partial y_{\lambda-1}} .
$$

By direct elementary computations, for $\kappa=1$ and for $\kappa=2$, we obtain the following two very classical formulas :

$$
\left\{\begin{aligned}
\mathbf{Y}_{1}= & \mathcal{Y}_{x}+\left[\mathcal{Y}_{y}-\mathcal{X}_{x}\right] y_{1}+\left[-\mathcal{X}_{y}\right]\left(y_{1}\right)^{2} \\
\mathbf{Y}_{2}= & \mathcal{Y}_{x^{2}}+\left[2 \mathcal{Y}_{x y}-\mathcal{X}_{x^{2}}\right] y_{1}+\left[\mathcal{Y}_{y^{2}}-2 \mathcal{X}_{x y}\right]\left(y_{1}\right)^{2}+\left[-\mathcal{X}_{y^{2}}\right]\left(y_{1}\right)^{3}+ \\
& +\left[\mathcal{Y}_{y}-2 \mathcal{X}_{x}\right] y_{2}+\left[-3 \mathcal{X}_{y}\right] y_{1} y_{2} .
\end{aligned}\right.
$$

Our main objective is to devise the general combinatorics. Thus, to attain this aim, we have to achieve patiently formal computations of the next coefficients $\mathbf{Y}_{3}, \mathbf{Y}_{4}$ and $\mathbf{Y}_{5}$. We systematically use parentheses [.] to single out every coefficient of the polynomials $\mathbf{Y}_{3}$, $\mathbf{Y}_{4}$ and $\mathbf{Y}_{5}$ in the pure jet variables $y_{1}, y_{2}, y_{3}, y_{4}$ and $y_{5}$, putting every sign inside these parentheses. We always put the monomials in the pure jet variables $y_{1}, y_{2}, y_{3}, y_{4}$ and $y_{5}$ after the parentheses. For completeness, let us provide the intermediate computation of the third coefficient $\mathbf{Y}_{3}$. In detail:

$$
\begin{aligned}
& \mathbf{Y}_{3}=D^{3}\left(\mathbf{Y}_{2}\right)-D^{1}(\mathcal{X}) y_{3} \\
& =\left(\frac{\partial}{\partial x}+y_{1} \frac{\partial}{\partial y}+y_{2} \frac{\partial}{\partial y_{1}}+y_{3} \frac{\partial}{\partial y_{2}}\right)\left(\mathcal{Y}_{x^{2}}+\left[2 \mathcal{Y}_{x y}-\mathcal{X}_{x^{2}}\right] y_{1}+\right. \\
& +\left[\mathcal{Y}_{y^{2}}-2 \mathcal{X}_{x y}\right]\left(y_{1}\right)^{2}+\left[-\mathcal{X}_{y^{2}}\right]\left(y_{1}\right)^{3}+ \\
& \left.+\left[\mathcal{Y}_{y}-2 \mathcal{X}_{x}\right] y_{2}+\left[-3 \mathcal{X}_{y}\right] y_{1} y_{2}\right) \\
& =\underline{\mathcal{Y}_{x^{3}}} \underline{1}^{+} \underline{\left[2 \mathcal{Y}_{x^{2} y}-\mathcal{X}_{x^{3}}\right] y_{1}{ }_{2}}+\underline{\left.\left[\mathcal{Y}_{x y^{2}}-2 \mathcal{X}_{x^{2} y}\right]\left(y_{1}\right)^{2}\right]_{3}}+\underline{\left[-\mathcal{X}_{x y^{2}}\right]\left(y_{1}\right)^{3}{ }_{4}}+ \\
& \left.+\underline{\left[\mathcal{Y}_{x y}-2 \mathcal{X}_{x^{2}}\right] y_{2}} \underline{6}+\underline{\left[-3 \mathcal{X}_{x y}\right] y_{1} y_{2}} 7\right]+\underline{\left.\mathcal{Y}_{x^{2} y}\right] y_{1}}{ }^{+} \\
& +\left[2 \mathcal{Y}_{x y^{2}}-\mathcal{X}_{x^{2} y}\right]\left(y_{1}\right)^{2}{ }_{3}+\left[\mathcal{Y}_{y^{3}}-2 \mathcal{X}_{x y^{2}}\right]\left(y_{1}\right)^{3}{ }_{4}+\left[-\mathcal{X}_{y^{3}}\right]\left(y_{1}\right)^{4}{ }_{5}^{+}
\end{aligned}
$$




$$
\begin{aligned}
& +\underline{\left[\mathcal{Y}_{y^{2}}-2 \mathcal{X}_{x y}\right] y_{1} y_{2}} \underline{7}+\underline{\left[-3 \mathcal{X}_{y^{2}}\right]\left(y_{1}\right)^{2} y_{2}}{ }_{8}+\underline{\left[2 \mathcal{Y}_{x y}-\mathcal{X}_{x^{2}}\right] y_{2}}{ }_{6}^{+} \\
& \left.\left.\left.+\underline{\left[\mathcal{Y}_{y^{2}}-2 \mathcal{X}_{x y}\right] 2 y_{1} y_{2}} 7\right]^{+\left[-\mathcal{X}_{y^{2}}\right] 3\left(y_{1}\right)^{2} y_{2}} 8\right]^{+\left[-3 \mathcal{X}_{y}\right]\left(y_{2}\right)^{2}}\right]^{+} \\
& +\left[\mathcal{Y}_{y}-2 \mathcal{X}_{x}\right] y_{3}{ }_{10}+\left[-3 \mathcal{X}_{y}\right] y_{1} y_{3}{ }_{11}^{-} \\
& -\left[\mathcal{X}_{x}\right] y_{3} 10-\left[\mathcal{X}_{y}\right] y_{1} y_{3}
\end{aligned}
$$

We have underlined all the terms with a number appended. Each number refers to the order of appearance of the terms in the final simplified expression of $\mathbf{Y}_{3}$, also written in [BK1989] with different notations:

$$
\left\{\begin{aligned}
\mathbf{Y}_{3}= & \mathcal{Y}_{x^{3}}+\left[3 \mathcal{Y}_{x^{2} y}-\mathcal{X}_{x^{3}}\right] y_{1}+\left[3 \mathcal{Y}_{x y^{2}}-3 \mathcal{X}_{x^{2} y}\right]\left(y_{1}\right)^{2}+ \\
& +\left[\mathcal{Y}_{y^{3}}-3 \mathcal{X}_{x y^{2}}\right]\left(y_{1}\right)^{3}+\left[-\mathcal{X}_{y^{3}}\right]\left(y_{1}\right)^{4}+\left[3 \mathcal{Y}_{x y}-3 \mathcal{X}_{x^{2}}\right] y_{2}+ \\
& +\left[3 \mathcal{Y}_{y^{2}}-9 \mathcal{X}_{x y}\right] y_{1} y_{2}+\left[-6 \mathcal{X}_{y^{2}}\right]\left(y_{1}\right)^{2} y_{2}+\left[-3 \mathcal{X}_{y}\right]\left(y_{2}\right)^{2}+ \\
& +\left[\mathcal{Y}_{y}-3 \mathcal{X}_{x}\right] y_{3}+\left[-4 \mathcal{X}_{y}\right] y_{1} y_{3} .
\end{aligned}\right.
$$

After similar manual computations, the intermediate details of which we will not copy in this Latex file, we get the desired expressions of $\mathbf{Y}_{4}$ and of $\mathbf{Y}_{5}$. Firstly:

$$
\left\{\begin{aligned}
\mathbf{Y}_{4}= & \mathcal{Y}_{x^{4}}+\left[4 \mathcal{Y}_{x^{3} y}-\mathcal{X}_{x^{4}}\right] y_{1}+\left[6 \mathcal{Y}_{x^{2} y^{2}}-4 \mathcal{X}_{x^{3} y}\right]\left(y_{1}\right)^{2}+ \\
& +\left[4 \mathcal{Y}_{x y^{3}}-6 \mathcal{X}_{x^{2} y^{2}}\right]\left(y_{1}\right)^{3}+\left[\mathcal{Y}_{y^{4}}-4 \mathcal{X}_{x y^{3}}\right]\left(y_{1}\right)^{4}+\left[-\mathcal{X}_{y^{4}}\right]\left(y_{1}\right)^{5}+ \\
& +\left[6 \mathcal{Y}_{x^{2} y}-4 \mathcal{X}_{x^{3}}\right] y_{2}+\left[12 \mathcal{Y}_{x y^{2}}-18 \mathcal{X}_{x^{2} y}\right] y_{1} y_{2}+ \\
& +\left[6 \mathcal{Y}_{y^{3}}-24 \mathcal{X}_{x y^{2}}\right]\left(y_{1}\right)^{2} y_{2}+\left[-10 \mathcal{X}_{y^{3}}\right]\left(y_{1}\right)^{3} y_{2}+ \\
& +\left[3 \mathcal{Y}_{y^{2}}-12 \mathcal{X}_{x y}\right]\left(y_{2}\right)^{2}+\left[-15 \mathcal{X}_{y^{2}}\right] y_{1}\left(y_{2}\right)^{2}+ \\
& +\left[4 \mathcal{Y}_{x y}-6 \mathcal{X}_{x^{2}}\right] y_{3}+\left[4 \mathcal{Y}_{y^{2}}-16 \mathcal{X}_{x y}\right] y_{1} y_{3}+\left[-10 \mathcal{X}_{y^{2}}\right]\left(y_{1}\right)^{2} y_{3}+ \\
& +\left[-10 \mathcal{X}_{y}\right] y_{2} y_{3}+\left[\mathcal{Y}_{y}-4 \mathcal{X}_{x}\right] y_{4}+\left[-5 \mathcal{X}_{y}\right] y_{1} y_{4}
\end{aligned}\right.
$$

Secondly:

$$
\left\{\begin{aligned}
\mathbf{Y}_{5}= & \mathcal{Y}_{x^{5}}+\left[5 \mathcal{Y}_{x^{4} y}-\mathcal{X}_{x^{5}}\right] y_{1}+\left[10 \mathcal{Y}_{x^{3} y^{2}}-5 \mathcal{X}_{x^{4} y}\right]\left(y_{1}\right)^{2}+ \\
& +\left[10 \mathcal{Y}_{x^{2} y^{3}}-10 \mathcal{X}_{x^{3} y^{2}}\right]\left(y_{1}\right)^{3}+\left[5 \mathcal{Y}_{x y^{4}}-10 \mathcal{X}_{x^{2} y^{3}}\right]\left(y_{1}\right)^{4}+ \\
& +\left[\mathcal{Y}_{y^{5}}-5 \mathcal{X}_{x y^{4}}\right]\left(y_{1}\right)^{5}+\left[-\mathcal{X}_{y^{5}}\right]\left(y_{1}\right)^{6}+\left[10 \mathcal{Y}_{x^{3} y}-5 \mathcal{X}_{x^{4}}\right] y_{2}+ \\
& +\left[30 \mathcal{Y}_{x^{2} y^{2}}-30 \mathcal{X}_{x^{3} y}\right] y_{1} y_{2}+\left[30 \mathcal{Y}_{x y^{3}}-60 \mathcal{X}_{x^{2} y^{2}}\right]\left(y_{1}\right)^{2} y_{2}+ \\
& +\left[10 \mathcal{Y}_{y^{4}}-50 \mathcal{X}_{x y^{3}}\right]\left(y_{1}\right)^{3} y_{2}+\left[-15 \mathcal{X}_{y^{4}}\right]\left(y_{1}\right)^{4} y_{2}+ \\
& +\left[15 \mathcal{Y}_{x y^{2}}-30 \mathcal{X}_{x^{2} y}\right]\left(y_{2}\right)^{2}+\left[15 \mathcal{Y}_{y^{3}}-75 \mathcal{X}_{x y^{2}}\right] y_{1}\left(y_{2}\right)^{2}+ \\
& +\left[-45 \mathcal{X}_{y^{3}}\right]\left(y_{1}\right)^{2}\left(y_{2}\right)^{2}+\left[-15 \mathcal{X}_{y^{2}}\right]\left(y_{2}\right)^{3}+ \\
& +\left[10 \mathcal{Y}_{x^{2} y}-10 \mathcal{X}_{x^{3}}\right] y_{3}+\left[20 \mathcal{Y}_{x y^{2}}-40 \mathcal{X}_{x^{2} y}\right] y_{1} y_{3}+ \\
& +\left[10 \mathcal{Y}_{y^{3}}-50 \mathcal{X}_{x y^{2}}\right]\left(y_{1}\right)^{2} y_{3}+\left[-20 \mathcal{X}_{y^{3}}\right]\left(y_{1}\right)^{3} y_{3}+ \\
& +\left[10 \mathcal{Y}_{y^{2}}-50 \mathcal{X}_{x y}\right] y_{2} y_{3}+\left[-60 \mathcal{X}_{y^{2}}\right] y_{1} y_{2} y_{3}+\left[-10 \mathcal{X}_{y}\right]\left(y_{3}\right)^{2}+ \\
& +\left[5 \mathcal{Y}_{x y}-10 \mathcal{X}_{x^{2}}\right] y_{4}+\left[5 \mathcal{Y}_{y^{2}}-25 \mathcal{X}_{x y}\right] y_{1} y_{4}+\left[-15 \mathcal{X}_{y^{2}}\right]\left(y_{1}\right)^{2} y_{4}+ \\
& +\left[-15 \mathcal{X}_{y}\right] y_{2} y_{4}+\left[\mathcal{Y}_{y}-5 \mathcal{X}_{y}\right] y_{5}+\left[-6 \mathcal{X}_{y}\right] y_{1} y_{5} .
\end{aligned}\right.
$$

2.11. Formal inspection, formal intuition and formal induction. Now, we have to comment these formulas. We have written in length the five polynomials $\mathbf{Y}_{1}, \mathbf{Y}_{2}, \mathbf{Y}_{3}$, $\mathbf{Y}_{4}$ and $\mathbf{Y}_{5}$ in the pure jet variables $y_{1}, y_{2}, y_{3}, y_{4}$ and $y_{5}$. Except the first "constant" term $\mathcal{Y}_{x^{\kappa}}$, all the monomials in the expression of $\mathbf{Y}_{\kappa}$ are of the general form

$$
\left(y_{\lambda_{1}}\right)^{\mu_{1}}\left(y_{\lambda_{2}}\right)^{\mu_{2}} \cdots\left(y_{\lambda_{d}}\right)^{\mu_{d}}
$$


for some positive integer $d \geqslant 1$, for some collection of strictly increasing jet indices:

$$
1 \leqslant \lambda_{1}<\lambda_{2}<\cdots<\lambda_{d} \leqslant \kappa,
$$

and for some positive integers $\mu_{1}, \ldots, \mu_{d} \geqslant 1$. This and the next combinatorial facts may be confirmed by reading the formulas giving $\mathbf{Y}_{1}, \mathbf{Y}_{2}, \mathbf{Y}_{3}, \mathbf{Y}_{4}$ and $\mathbf{Y}_{5}$. It follows that the integer $d$ satisfies the inequality $d \leqslant \kappa+1$. To include the first "constant" term $\mathcal{Y}_{x^{\kappa}}$, we shall make the convention that putting $d=0$ in the monomial (2.12) yields the constant term 1.

Furthermore, by inspecting the formulas giving $\mathbf{Y}_{1}, \mathbf{Y}_{2}, \mathbf{Y}_{3}, \mathbf{Y}_{4}$ and $\mathbf{Y}_{5}$, we see that the following inequality should be satisfied:

$$
\mu_{1} \lambda_{1}+\mu_{2} \lambda_{2}+\cdots+\mu_{d} \lambda_{d} \leqslant \kappa+1
$$

For instance, in the expression of $\mathbf{Y}_{4}$, the two monomials $\left(y_{1}\right)^{3} y_{2}$ and $y_{1}\left(y_{2}\right)^{2}$ do appear, but the two monomials $\left(y_{1}\right)^{4} y_{2}$ and $\left(y_{1}\right)^{2}\left(y_{2}\right)^{2}$ cannot appear. All coefficients of the pure jet monomials are of the general form:

$$
\left[A \mathcal{Y}_{x^{\alpha} y^{\beta+1}}-B \mathcal{X}_{x^{\alpha+1} y^{\beta}}\right]
$$

for some nonnegative integers $A, B, \alpha, \beta \in \mathbb{N}$. Sometimes $A$ is zero, but $B$ is zero only for the (constant, with respect to pure jet variables) term $\mathcal{Y}_{x^{\kappa}}$. Importantly, $\mathcal{X}$ is differentiated once more with respect to $x$ and $\mathcal{Y}$ is differentiated once more with respect to $y$. Again, this may be confirmed by reading all the terms in the formulas for $\mathbf{Y}_{1}, \mathbf{Y}_{2}, \mathbf{Y}_{3}, \mathbf{Y}_{4}$ and $\mathbf{Y}_{5}$.

In addition, we claim that there is a link between the couple $(\alpha, \beta)$ and the collection $\left\{\mu_{1}, \lambda_{1}, \ldots, \mu_{d}, \lambda_{d}\right\}$. To discover it, let us write some of the monomials appearing in the expressions of $\mathbf{Y}_{4}$ (first column) and of $\mathbf{Y}_{5}$ (second column), for instance:

$$
\left\{\begin{array}{lr}
{\left[6 \mathcal{Y}_{x^{2} y^{2}}-4 \mathcal{X}_{x^{3} y}\right]\left(y_{1}\right)^{2},} & {\left[5 \mathcal{Y}_{x y^{4}}-10 \mathcal{X}_{x^{2} y^{3}}\right]\left(y_{1}\right)^{4},} \\
{\left[12 \mathcal{Y}_{x y^{2}}-18 \mathcal{X}_{x^{2} y}\right] y_{1} y_{2},} & {\left[30 \mathcal{Y}_{x y^{3}}-60 \mathcal{X}_{x^{2} y^{2}}\right]\left(y_{1}\right)^{2} y_{2},} \\
{\left[-10 \mathcal{X}_{y^{3}}\right]\left(y_{1}\right)^{3} y_{2},} & {\left[-15 \mathcal{X}_{y^{4}}\right]\left(y_{1}\right)^{4} y_{2},} \\
{\left[4 \mathcal{Y}_{y^{2}}-16 \mathcal{X}_{x y}\right] y_{1} y_{3},} & {\left[10 \mathcal{Y}_{y^{2}}-50 \mathcal{X}_{x y}\right] y_{2} y_{3},} \\
{\left[-10 \mathcal{X}_{y^{2}}\right]\left(y_{1}\right)^{2} y_{3},} & {\left[-60 \mathcal{X}_{y^{2}}\right] y_{1} y_{2} y_{3}}
\end{array}\right.
$$

After some reflection, we discover the hidden intuitive rule: the partial derivatives of $\mathcal{Y}$ and of $\mathcal{X}$ associated with the monomial $\left(y_{\lambda_{1}}\right)^{\mu_{1}} \cdots\left(y_{\lambda_{d}}\right)^{\mu_{d}}$ are, respectively:

$$
\left\{\begin{array}{l}
\mathcal{Y}_{x^{\kappa-\mu_{1} \lambda_{1}-\cdots-\mu_{d} \lambda_{d}} y^{\mu_{1}+\cdots+\mu_{d}}}, \\
\mathcal{X}_{x^{\kappa-\mu_{1} \lambda_{1}-\cdots-\mu_{d} \lambda_{d}+1} y^{\mu_{1}+\cdots+\mu_{d}-1}}
\end{array}\right.
$$

This may be checked on each of the 10 examples (2.16) above.

Now that we have explored and discovered the combinatorics of the pure jet monomials, of the partial derivatives and of the complete sum giving $\mathbf{Y}_{\kappa}$, we may express that it is of the following general form:

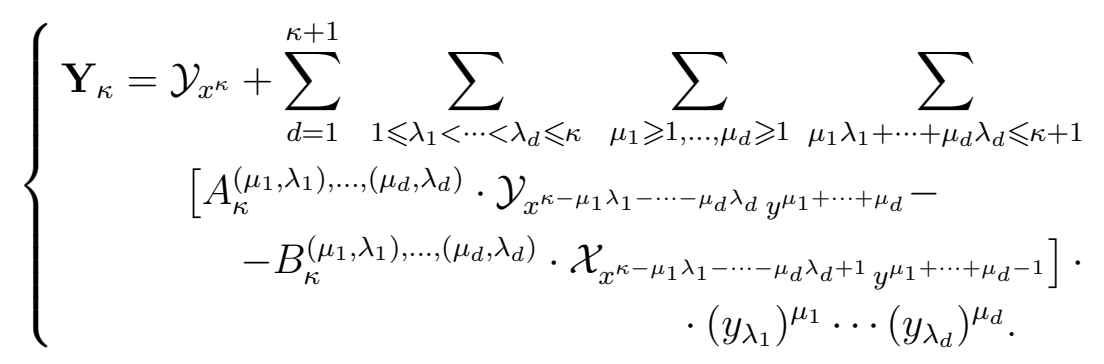

Here, we separate the first term $\mathcal{Y}_{x^{\kappa}}$ from the general sum; it is the constant term in $\mathbf{Y}_{\kappa}$, which itself is a polynomial with respect to the jet variables $y_{\lambda}$. In this general formula, the 
only remaining unknowns are the nonnegative integer coefficients $A_{\kappa}^{\left(\mu_{1}, \lambda_{1}\right), \ldots,\left(\mu_{d}, \lambda_{d}\right)} \in \mathbb{N}$ and $B_{\kappa}^{\left(\mu_{1}, \lambda_{1}\right), \ldots,\left(\mu_{d}, \lambda_{d}\right)} \in \mathbb{N}$. In Section 3 below, we shall explain how we have discovered their exact value.

At present, even if we are unable to devise their explicit expression, we may observe that the value of the special integer coefficients $A_{\mu_{1}}^{\left(\mu_{1}, 1\right)}$ and $B_{\mu_{1}}^{\left(\mu_{1}, 1\right)}$ which are attached to the monomials ct., $y_{1},\left(y_{1}\right)^{2},\left(y_{1}\right)^{3},\left(y_{1}\right)^{4}$ and $\left(y_{1}\right)^{5}$ are simple. Indeed, by inspecting the first terms in the expressions of $\mathbf{Y}_{1}, \mathbf{Y}_{2}, \mathbf{Y}_{3}, \mathbf{Y}_{4}$ and $\mathbf{Y}_{5}$, we of course recognize the binomial coefficients. In general:

Lemma 2.19. For $\kappa \geqslant 1$,

$$
\left\{\begin{aligned}
\mathbf{Y}_{\kappa}= & \mathcal{Y}_{x^{\kappa}}+\sum_{\lambda=1}^{\kappa}\left[\left(\begin{array}{c}
\kappa \\
\lambda
\end{array}\right) \mathcal{Y}_{x^{\kappa-\lambda} y^{\lambda}}-\left(\begin{array}{c}
\kappa \\
\lambda-1
\end{array}\right) \mathcal{X}_{x^{\kappa-\lambda+1} y^{\lambda-1}}\right]\left(y_{1}\right)^{\lambda}+ \\
& +\left[-\mathcal{X}_{y^{\kappa}}\right]\left(y_{1}\right)^{\kappa}+\text { remainder }
\end{aligned}\right.
$$

where the term remainder collects all remaining monomials in the pure jet variables.

In addition, let us remind what we have observed and used in a previous co-signed work.

Lemma 2.21. ([GM2003a], p. 536) For $\kappa \geqslant 4$, nine among the monomials of $\mathbf{Y}_{\kappa}$ are of the following general form:

$$
\left\{\begin{aligned}
\mathbf{Y}_{\kappa}= & \mathcal{Y}_{x^{\kappa}}+\left[C_{\kappa}^{1} \mathcal{Y}_{x^{\kappa-1} y}-\mathcal{X}_{x^{\kappa}}\right] y_{1}+\left[C_{\kappa}^{2} \mathcal{Y}_{x^{\kappa-2} y}-C_{\kappa}^{1} \mathcal{X}_{x^{\kappa-1}}\right] y_{2}+ \\
& +\left[C_{\kappa}^{2} \mathcal{Y}_{x^{2} y}-C_{\kappa}^{3} \mathcal{X}_{x^{3}}\right] y_{\kappa-2}+\left[C_{\kappa}^{1} \mathcal{Y}_{x y}-C_{\kappa}^{2} \mathcal{X}_{x^{2}}\right] y_{\kappa-1}+ \\
& +\left[C_{\kappa}^{1} \mathcal{Y}_{y^{2}}-\kappa^{2} \mathcal{X}_{x y}\right] y_{1} y_{\kappa-1}+\left[-C_{\kappa}^{2} \mathcal{X}_{y}\right] y_{2} y_{\kappa-1}+ \\
& +\left[\mathcal{Y}_{y}-C_{\kappa}^{1} \mathcal{X}_{x}\right]+\left[-C_{\kappa+1}^{1} \mathcal{X}_{y}\right] y_{1} y_{\kappa}+\text { remainder }
\end{aligned}\right.
$$

where the term remainder denotes all the remaining monomials, and where $C_{\kappa}^{\lambda}:=$ $\frac{\kappa !}{(\kappa-\lambda) ! \lambda !}$ is a notation for the binomial coefficient which occupies less space in Latex "equation mode" than the classical notation

$$
\left(\begin{array}{l}
\kappa \\
\lambda
\end{array}\right) .
$$

Now, we state directly the final theorem, without further inductive or intuitive information.

Theorem 2.24. For $\kappa \geqslant 1$, we have:

$$
\begin{gathered}
\mathbf{Y}_{\kappa}=\mathcal{Y}_{x^{\kappa}}+\sum_{d=1}^{\kappa+1} \sum_{1 \leqslant \lambda_{1}<\cdots<\lambda_{d} \leqslant \kappa} \sum_{\mu_{1} \geqslant 1, \ldots, \mu_{d} \geqslant 1} \sum_{\mu_{1} \lambda_{1}+\cdots+\mu_{d} \lambda_{d} \leqslant \kappa+1} \\
{\left[\frac{\kappa \cdots\left(\kappa-\mu_{1} \lambda_{1}-\cdots-\mu_{d} \lambda_{d}+1\right)}{\left(\lambda_{1} !\right)^{\mu_{1}} \mu_{1} ! \cdots\left(\lambda_{d} !\right)^{\mu_{d}} \mu_{d} !} \cdot \mathcal{Y}_{x^{\kappa-\mu_{1} \lambda_{1}-\cdots-\mu_{d} \lambda_{d}} y^{\mu_{1}+\cdots+\mu_{d}}-}\right.} \\
-\frac{\kappa \cdots\left(\kappa-\mu_{1} \lambda_{1}-\cdots-\mu_{d} \lambda_{d}+2\right)\left(\mu_{1} \lambda_{1}+\cdots+\mu_{d} \lambda_{d}\right)}{\left(\lambda_{1} !\right)^{\mu_{1}} \mu_{1} ! \cdots\left(\lambda_{d} !\right)^{\mu_{d}} \mu_{d} !} \\
\cdot \mathcal{X}_{\left.x^{\kappa-\mu_{1} \lambda_{1}-\cdots-\mu_{d} \lambda_{d}+1} y^{\mu_{1}+\cdots+\mu_{d}-1}\right]\left(y_{\lambda_{1}}\right)^{\mu_{1}} \cdots\left(y_{\lambda_{d}}\right)^{\mu_{d}}}
\end{gathered}
$$


Once the correct theorem is formulated, its proof follows by accessible induction arguments which will not be developed here. It is better to continue through and to examine thorougly the case of several variables, since it will help us considerably to explain how we discovered the exact values of the integer coefficients $A_{\kappa}^{\left(\mu_{1}, \lambda_{1}\right), \ldots,\left(\mu_{d}, \lambda_{d}\right)}$ and $B_{\kappa}^{\left(\mu_{1}, \lambda_{1}\right), \ldots,\left(\mu_{d}, \lambda_{d}\right)}$.

2.26. Verification and application. Before proceeding further, let us rapidly verify that the above general formula (2.25) is correct by inspecting two instances extracted from $\mathbf{Y}_{5}$.

Firstly, the coefficient of $\left(y_{1}\right)^{3} y_{3}$ in $\mathbf{Y}_{5}$ is obtained by putting $\kappa=5, d=2, \lambda_{1}=1$, $\mu_{1}=3, \lambda_{2}=3$ and $\mu_{2}=1$ in the general formula (2.25), which yields:

$$
\left[0-\frac{5 \cdot 4 \cdot 3 \cdot 2 \cdot 1 \cdot 6}{(1 !)^{3} 3 !(3 !)^{1} 1 !} \mathcal{X}_{y^{3}}\right]=\left[-20 \mathcal{X}_{y^{3}}\right]
$$

This value is the same as in the original formula (2.10): confirmation.

Secondly, the coefficient of $y_{1}\left(y_{2}\right)^{2}$ in $\mathbf{Y}_{5}$ is obtained by $\kappa=5, d=2, \lambda_{1}=1, \mu_{1}=1$, $\lambda_{2}=2$ and $\mu_{2}=2$ in the general formula (2.25), which yields:

$$
\left[\frac{5 \cdot 4 \cdot 3 \cdot 2 \cdot 1}{(1 !)^{1} 1 !(2 !)^{2} 2 !} \mathcal{Y}_{y^{3}}-\frac{5 \cdot 4 \cdot 3 \cdot 2 \cdot 5}{(1 !)^{1} 1 !(2 !)^{2} 2 !} \mathcal{X}_{x y^{2}}\right]=\left[15 \mathcal{Y}_{y^{3}}-75 \mathcal{X}_{x y^{2}}\right]
$$

This value is the same as in the original formula (2.10); again: confirmation.

Finally, applying our general formula (2.25), we deduce the value of $\mathbf{Y}_{6}$ without having to use $\mathbf{Y}_{5}$ and the induction formulas (2.4), which shortens substantially the computations.

For the pleasure, we obtain:

$$
\begin{aligned}
& \left(\mathbf{Y}_{6}=\mathcal{Y}_{x^{6}}+\left[6 \mathcal{Y}_{x^{5} y}-\mathcal{X}_{x^{6}}\right] y_{1}+\left[15 \mathcal{Y}_{x^{4} y^{2}}-6 \mathcal{X}_{x^{5} y}\right]\left(y_{1}\right)^{2}+\right. \\
& +\left[20 \mathcal{Y}_{x^{3} y^{3}}-15 \mathcal{X}_{x^{4} y^{2}}\right]\left(y_{1}\right)^{3}+\left[15 \mathcal{Y}_{x^{2} y^{4}}-20 \mathcal{X}_{x^{3} y^{3}}\right]\left(y_{1}\right)^{4}+ \\
& +\left[6 \mathcal{Y}_{x y^{5}}-15 \mathcal{X}_{x^{2} y^{4}}\right]\left(y_{1}\right)^{5}+\left[\mathcal{Y}_{y^{6}}-6 \mathcal{X}_{x y^{5}}\right]\left(y_{1}\right)^{6}+\left[-\mathcal{X}_{y^{6}}\right]\left(y_{1}\right)^{7}+ \\
& +\left[15 \mathcal{Y}_{x^{4} y}-6 \mathcal{X}_{x^{5}}\right] y_{2}+\left[60 \mathcal{Y}_{x^{3} y^{2}}-45 \mathcal{X}_{x^{4} y}\right] y_{1} y_{2}+ \\
& +\left[90 \mathcal{Y}_{x^{2} y^{3}}-120 \mathcal{X}_{x^{3} y^{2}}\right]\left(y_{1}\right)^{2} y_{2}+\left[60 \mathcal{Y}_{x y^{4}}-150 \mathcal{X}_{x^{2} y^{3}}\right]\left(y_{1}\right)^{3} y_{2}+ \\
& +\left[15 \mathcal{Y}_{y^{5}}-90 \mathcal{X}_{x y^{4}}\right]\left(y_{1}\right)^{4} y_{2}+\left[-21 \mathcal{X}_{y^{5}}\right]\left(y_{1}\right)^{5} y_{2}+ \\
& +\left[45 \mathcal{Y}_{x^{2} y^{2}}-60 \mathcal{X}_{x^{3} y}\right]\left(y_{2}\right)^{2}+\left[90 \mathcal{Y}_{x y^{3}}-225 \mathcal{X}_{x^{2} y^{2}}\right] y_{1}\left(y_{2}\right)^{2}+ \\
& +\left[45 \mathcal{Y}_{y^{4}}-270 \mathcal{X}_{x y^{3}}\right]\left(y_{1}\right)^{2}\left(y_{2}\right)^{2}+\left[-210 \mathcal{X}_{y^{4}}\right]\left(y_{1}\right)^{3}\left(y_{2}\right)^{2}+ \\
& +\left[15 \mathcal{Y}_{y^{3}}-90 \mathcal{X}_{x y^{2}}\right]\left(y_{2}\right)^{3}+\left[-105 \mathcal{X}_{y^{3}}\right] y_{1}\left(y_{2}\right)^{3}+ \\
& +\left[20 \mathcal{Y}_{x^{3} y}-15 \mathcal{X}_{x^{4}}\right] y_{3}+\left[60 \mathcal{Y}_{x^{2} y^{2}}-80 \mathcal{X}_{x^{3} y}\right] y_{1} y_{3}+ \\
& +\left[60 \mathcal{Y}_{x y^{3}}-150 \mathcal{X}_{x^{2} y^{2}}\right]\left(y_{1}\right)^{2} y_{3}+\left[20 \mathcal{Y}_{y^{4}}-120 \mathcal{X}_{x y^{3}}\right]\left(y_{1}\right)^{3} y_{3}+ \\
& +\left[-35 \mathcal{X}_{y^{4}}\right]\left(y_{1}\right)^{4} y_{3}+\left[60 \mathcal{Y}_{x y^{2}}-150 \mathcal{X}_{x^{2} y}\right] y_{2} y_{3}+ \\
& +\left[60 \mathcal{Y}_{y^{3}}-360 \mathcal{X}_{x y^{2}}\right] y_{1} y_{2} y_{3}+\left[-210 \mathcal{X}_{y^{3}}\right]\left(y_{1}\right)^{2} y_{2} y_{3}+ \\
& +\left[-105 \mathcal{X}_{y^{2}}\right]\left(y_{2}\right)^{2} y_{3}+\left[10 \mathcal{Y}_{y^{2}}-60 \mathcal{X}_{x y}\right]\left(y_{3}\right)^{2}+ \\
& +\left[-70 \mathcal{X}_{y^{2}}\right] y_{1}\left(y_{3}\right)^{2}+\left[15 \mathcal{Y}_{x^{2} y}-20 \mathcal{X}_{x^{3}}\right] y_{4}+ \\
& +\left[30 \mathcal{Y}_{x y^{2}}-75 \mathcal{X}_{x^{2} y}\right] y_{1} y_{4}+\left[15 \mathcal{Y}_{y^{3}}-90 \mathcal{X}_{x y^{2}}\right]\left(y_{1}\right)^{2} y_{4}+ \\
& +\left[-35 \mathcal{X}_{y^{3}}\right]\left(y_{1}\right)^{3} y_{4}+\left[15 \mathcal{Y}_{y^{2}}-90 \mathcal{X}_{x y}\right] y_{2} y_{4}+ \\
& +\left[-105 \mathcal{X}_{y^{2}}\right] y_{1} y_{2} y_{4}+\left[-35 \mathcal{X}_{y}\right] y_{3} y_{4}+\left[6 \mathcal{Y}_{x y}-15 \mathcal{X}_{x^{2}}\right] y_{5}+ \\
& +\left[6 \mathcal{Y}_{y^{2}}-36 \mathcal{X}_{x y}\right] y_{1} y_{5}+\left[-21 \mathcal{X}_{y^{2}}\right]\left(y_{1}\right)^{2} y_{5}+\left[-21 \mathcal{X}_{y}\right] y_{2} y_{5}+ \\
& +\left[\mathcal{Y}_{y}-6 \mathcal{X}_{y}\right] y_{6}+\left[-7 \mathcal{X}_{y}\right] y_{1} y_{6} \text {. }
\end{aligned}
$$


2.30. Deduction of the classical Faà di Bruno formula. Let $x, y \in \mathbb{K}$ and let $g=g(x)$, $f=f(y)$ be two $\mathcal{C}^{\infty}$-smooth functions $\mathbb{K} \rightarrow \mathbb{K}$. Consider the composition $h:=f \circ g$, namely $h(x)=f(g(x))$. For $\lambda \in \mathbb{N}$ with $\lambda \geqslant 1$, simply denote by $g_{\lambda}$ the $\lambda$-th derivative $\frac{d^{\lambda} g}{d x^{\lambda}}$ and similarly for $h_{\lambda}$. Also, abbreviate $f_{\lambda}:=\frac{d^{\lambda} f}{d y^{\lambda}}$.

By the classical formula for the derivative of a composite function, we have $h_{1}=f_{1} g_{1}$. Further computations provide the following list of subsequent derivatives of $h$ :

$$
\left\{\begin{aligned}
h_{1}= & f_{1} g_{1}, \\
h_{2}= & f_{2}\left(g_{1}\right)^{2}+f_{1} g_{2} \\
h_{3}= & f_{3}\left(g_{1}\right)^{3}+3 f_{2} g_{1} g_{2}+f_{1} g_{3} \\
h_{4}= & f_{4}\left(g_{1}\right)^{4}+6 f_{3}\left(g_{1}\right)^{2} g_{2}+3 f_{2}\left(g_{2}\right)^{2}+4 f_{2} g_{1} g_{3}+f_{1} g_{4}, \\
h_{5}= & f_{5}\left(g_{1}\right)^{5}+10 f_{4}\left(g_{1}\right)^{3} g_{2}+15 f_{3}\left(g_{1}\right)^{2} g_{3}+10 f_{3} g_{1}\left(g_{2}\right)^{2}+ \\
& +10 f_{2} g_{2} g_{3}+5 f_{2} g_{1} g_{4}+f_{1} g_{5}, \\
h_{6}= & f_{6}\left(g_{1}\right)^{6}+15 f_{5}\left(g_{1}\right)^{4} g_{2}+45 f_{4}\left(g_{1}\right)^{2}\left(g_{2}\right)^{2}+15 f_{3}\left(g_{2}\right)^{3}+ \\
& +20 f_{4}\left(g_{1}\right)^{3} g_{3}+60 f_{3} g_{1} g_{2} g_{3}+10 f_{2}\left(g_{3}\right)^{2}+15 f_{3}\left(g_{1}\right)^{2} g_{4}+ \\
& +15 f_{2} g_{2} g_{4}+6 f_{2} g_{1} g_{5}+f_{1} g_{6} .
\end{aligned}\right.
$$

Theorem 2.32. For every integer $\kappa \geqslant 1$, the $\kappa$-th derivative of the composite function $h=f \circ g$ may be expressed as an explicit polynomial in the partial derivatives of $f$ and of $g$ having integer coefficients:

$$
\begin{aligned}
\frac{d^{\kappa} h}{d x^{\kappa}}= & \sum_{d=1}^{\kappa} \sum_{1 \leqslant \lambda_{1}<\cdots<\lambda_{d} \leqslant \kappa} \sum_{\mu_{1} \geqslant 1, \ldots, \mu_{d} \geqslant 1} \sum_{\mu_{1} \lambda_{1}+\cdots+\mu_{d} \lambda_{d}=\kappa} \\
& \frac{\kappa !}{\left(\lambda_{1} !\right)^{\mu_{1}} \mu_{1} ! \cdots\left(\lambda_{d} !\right)^{\mu_{d}} \mu_{d} !} \frac{d^{\mu_{1}+\cdots+\mu_{d}} f}{d y^{\mu_{1}+\cdots+\mu_{d}}}\left(\frac{d^{\lambda_{1}} g}{d x^{\lambda_{1}}}\right)^{\mu_{1}} \cdots \cdots\left(\frac{d^{\lambda_{d}} g}{d x^{\lambda_{d}}}\right)^{\mu_{d}} .
\end{aligned}
$$

This is the classical Faà di Bruno formula. Interestingly, we observe that this formula is included as a subpart of the general formula for $\mathbf{Y}_{\kappa}$, after a suitable translation. Indeed, in the formulas for $\mathbf{Y}_{1}, \mathbf{Y}_{2}, \mathbf{Y}_{3}, \mathbf{Y}_{4}, \mathbf{Y}_{5}, \mathbf{Y}_{6}$ and in the general sum for $\mathbf{Y}_{\kappa}$, pick only the terms for which $\mu_{1} \lambda_{1}+\cdots+\mu_{d} \lambda_{d}=\kappa$ and drop $\mathcal{X}$, which yields:

$$
\begin{aligned}
& \sum_{d=1}^{\kappa} \sum_{1 \leqslant \lambda_{1}<\cdots<\lambda_{d} \leqslant \kappa} \sum_{\mu_{1} \geqslant 1, \ldots, \mu_{d} \geqslant 1} \sum_{\mu_{1} \lambda_{1}+\cdots+\mu_{d} \lambda_{d}=\kappa} \\
& {\left[\frac{\kappa !}{\mu_{1} !\left(\lambda_{1} !\right)^{\mu_{1}} \cdots \mu_{d} !\left(\lambda_{d} !\right)^{\mu_{d}}} \mathcal{Y}_{y^{\mu_{1}+\cdots+\mu_{d}}}\right]\left(y_{\lambda_{1}}\right)^{\mu_{1}} \cdots\left(y_{\lambda_{d}}\right)^{\mu_{d}} .}
\end{aligned}
$$

The similarity between the two formulas (2.33) and (2.34) is now clearly visible.

The Faà di Bruno formula may be established by means of substitutions of power series ([F1969], p. 222), by means of the umbral calculus ([CS1996]), or by means of some induction formulas, which we write for completeness. Define the differential operators (2.35)

$$
\begin{aligned}
& F_{2}:=g_{2} \frac{\partial}{\partial g_{1}}+g_{1}\left(f_{2} \frac{\partial}{\partial f_{1}}\right) \\
& F_{3}:=g_{2} \frac{\partial}{\partial g_{1}}+g_{3} \frac{\partial}{\partial g_{2}}+g_{1}\left(f_{2} \frac{\partial}{\partial f_{1}}+f_{3} \frac{\partial}{\partial f_{2}}\right) \\
& \ldots \ldots \ldots \ldots \ldots \ldots \ldots \ldots \cdots \cdots \cdots \cdots \cdots \cdots \cdots \cdots \cdots \cdots+g_{\lambda} \frac{\partial}{\partial g_{\lambda-1}}+g_{1}\left(f_{2} \frac{\partial}{\partial f_{1}}+f_{3} \frac{\partial}{\partial f_{2}}+\cdots+f_{\lambda} \frac{\partial}{\partial f_{\lambda-1}}\right) . \\
& F_{\lambda}:=g_{2} \frac{\partial}{\partial g_{1}}+g_{3} \frac{\partial}{\partial g_{2}}+\cdots \cdots
\end{aligned}
$$


Then we have

$$
\begin{aligned}
& h_{2}=F^{2}\left(h_{1}\right), \\
& h_{3}=F^{3}\left(h_{2}\right), \\
& \cdots \ldots \ldots \ldots \ldots \\
& h_{\lambda}=F^{\lambda}\left(h_{\lambda-1}\right) .
\end{aligned}
$$

\section{$\S 3$. SEVERAL INDEPENDENT VARIABLES AND ONE DEPENDENT VARIABLE}

3.1. Simplified adapted notations. As announced after the statement of Theorem 2.24 , it is only after we have treated the case of several independent variables that we will understand perfectly the general formula (2.25), valid in the case of one independent variable and one dependent variable. We will discover massive formal computations, exciting our computational intuition.

Thus, assume $n \geqslant 1$ and $m=1$, let $\kappa \in \mathbb{N}$ with $\kappa \geqslant 1$ and simply denote (instead of (1.2)) the jet variables by:

$$
\left(x^{i}, y, y_{i_{1}}, y_{i_{1}, i_{2}}, \ldots, y_{i_{1}, i_{2}, \ldots, i_{\kappa}}\right) .
$$

Also, instead of (1.30), denote the $\kappa$-th prolongation of a vector field by:

$$
\left\{\begin{array}{c}
\mathcal{L}^{(\kappa)}=\sum_{i=1}^{n} \mathcal{X}^{i} \frac{\partial}{\partial x^{i}}+\mathcal{Y} \frac{\partial}{\partial y}+\sum_{i_{1}=1}^{n} \mathbf{Y}_{i_{1}} \frac{\partial}{\partial y_{i_{1}}}+\sum_{i_{1}, i_{2}=1}^{n} \mathbf{Y}_{i_{1}, i_{2}} \frac{\partial}{\partial y_{i_{1}, i_{2}}}+ \\
+\cdots+\sum_{i_{1}, i_{2}, \ldots, i_{\kappa}=1}^{n} \mathbf{Y}_{i_{1}, i_{2}, \ldots, i_{\kappa}} \frac{\partial}{\partial y_{i_{1}, i_{2}, \ldots, i_{\kappa}}}
\end{array}\right.
$$

The induction formulas are

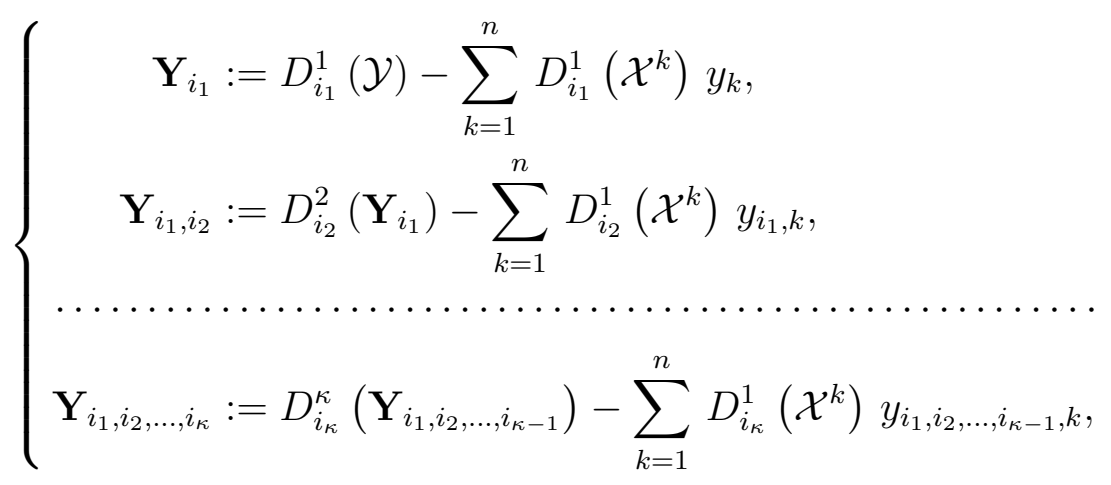

where the total differentiation operators $D_{i^{\prime}}^{\lambda}$ are defined as in (1.22), dropping the sums $\sum_{j^{\prime}=1}^{m}$ and the indices $j^{\prime}$.

3.5. Two instructing explicit computations. To begin with, let us compute $\mathbf{Y}_{i_{1}}$. With $D_{i_{1}}^{1}=\frac{\partial}{\partial x^{i_{1}}}+y_{i_{1}} \frac{\partial}{\partial y}$, we have:

$$
\begin{aligned}
\mathbf{Y}_{i_{1}} & =D_{i_{1}}(\mathcal{Y})-\sum_{k_{1}=1}^{n} D_{i_{1}}^{1}\left(\mathcal{X}^{k_{1}}\right) y_{k_{1}} \\
& =\mathcal{Y}_{x^{i_{1}}}+\mathcal{Y}_{y} y_{i_{1}}-\sum_{k_{1}=1}^{n} \mathcal{X}_{x^{i_{1}}}^{k_{1}} y_{k_{1}}-\sum_{k_{1}=1}^{n} \mathcal{X}_{y}^{k_{1}} y_{i_{1}} y_{k_{1}} .
\end{aligned}
$$


Searching for formal harmony and for coherence with the formula $(2.6)_{1}$, we must include the term $\mathcal{Y}_{y} y_{i_{1}}$ inside the sum $\sum_{k_{1}=1}^{n}[\cdot] y_{k_{1}}$. Using the Kronecker symbol, we may write:

$$
\mathcal{Y}_{y} y_{i_{1}} \equiv \sum_{k_{1}=1}^{n}\left[\delta_{i_{1}}^{k_{1}} \mathcal{Y}_{y}\right] y_{k_{1}}
$$

Also, we may rewrite the last term of (3.6) with a double sum:

$$
-\sum_{k_{1}=1}^{n} \mathcal{X}_{y}^{k_{1}} y_{i_{1}} y_{k_{1}} \equiv \sum_{k_{1}, k_{2}=1}^{n}\left[-\delta_{i_{1}}^{k_{1}} \mathcal{X}_{y}^{k_{2}}\right] y_{k_{1}} y_{k_{2}}
$$

From now on and up to equation (3.39), we shall abbreviate any sum $\sum_{k=1}^{n}$ from 1 to $n$ as $\sum_{k}$. Putting everything together, we get the final desired perfect expression of $\mathbf{Y}_{i_{1}}$ :

$$
\mathbf{Y}_{i_{1}}=\mathcal{Y}_{x^{i_{1}}}+\sum_{k_{1}}\left[\delta_{i_{1}}^{k_{1}} \mathcal{Y}_{y}-\mathcal{X}_{x^{i_{1}}}^{k_{1}}\right] y_{k_{1}}+\sum_{k_{1}, k_{2}}\left[-\delta_{i_{1}}^{k_{1}} \mathcal{X}_{y}^{k_{2}}\right] y_{k_{1}} y_{k_{2}}
$$

This completes the first explicit computation.

The second one is about $\mathbf{Y}_{i_{1}, i_{2}}$. It becomes more delicate, because several algebraic transformations must be achieved until the final satisfying formula is obtained. Our goal is to present each step very carefully, explaining every tiny detail. Without such a care, it would be impossible to claim that some of our subsequent computations, for which we will not provide the intermediate steps, may be redone and verified. Consequently, we will expose our rules of formal computation thoroughly.

Replacing the value of $\mathbf{Y}_{1}$ just obtained in the induction formula $(3.4)_{2}$ and developing, we may conduct the very first steps of the computation:

$$
\begin{aligned}
& \mathbf{Y}_{i_{1}, i_{2}}= D_{i_{2}}^{2}\left(\mathbf{Y}_{i_{1}}\right)-\sum_{k_{1}} D_{i_{2}}^{1}\left(\mathcal{X}^{k_{1}}\right) y_{i_{1}, k_{1}} \\
&=\left(\frac{\partial}{\partial x^{i_{2}}}+y_{i_{2}} \frac{\partial}{\partial y}+\sum_{k_{1}} y_{i_{2}, k_{1}} \frac{\partial}{\partial y_{k_{1}}}\right)\left(\mathcal{Y}_{x^{i_{1}}}+\sum_{k_{1}}\left[\delta_{i_{1}}^{k_{1}} \mathcal{Y}_{y}-\mathcal{X}_{x^{i_{1}}}^{k_{1}}\right] y_{k_{1}}+\right. \\
&\left.\quad+\sum_{k_{1}, k_{2}}\left[-\delta_{i_{1}}^{k_{1}} \mathcal{X}_{y}^{k_{2}}\right] y_{k_{1}} y_{k_{2}}\right)-\sum_{k_{1}}\left[\mathcal{X}_{x^{i_{2}}}^{k_{1}}+y_{i_{2}} \mathcal{X}_{y}^{k_{1}}\right] y_{i_{1}, k_{1}}
\end{aligned}
$$

$$
\begin{aligned}
= & \left(\frac{\partial}{\partial x^{i_{2}}}\right)\left(\mathcal{Y}_{x^{i_{1}}}+\sum_{k_{1}}\left[\delta_{i_{1}}^{k_{1}} \mathcal{Y}_{y}-\mathcal{X}_{x^{i_{1}}}^{k_{1}}\right] y_{k_{1}}+\sum_{k_{1}, k_{2}}\left[-\delta_{i_{1}}^{k_{1}} \mathcal{X}_{y}^{k_{2}}\right] y_{k_{1}} y_{k_{2}}\right)+ \\
& +\left(y_{i_{2}} \frac{\partial}{\partial y}\right)\left(\mathcal{Y}_{x^{i_{1}}}+\sum_{k_{1}}\left[\delta_{i_{1}}^{k_{1}} \mathcal{Y}_{y}-\mathcal{X}_{x^{i_{1}}}^{k_{1}}\right] y_{k_{1}}+\sum_{k_{1}, k_{2}}\left[-\delta_{i_{1}}^{k_{1}} \mathcal{X}_{y}^{k_{2}}\right] y_{k_{1}} y_{k_{2}}\right)+ \\
& +\left(\sum_{k_{1}} y_{i_{2}, k_{1}} \frac{\partial}{\partial y_{k_{1}}}\right)\left(\mathcal{Y}_{x^{i_{1}}}+\sum_{k_{1}}\left[\delta_{i_{1}}^{k_{1}} \mathcal{Y}_{y}-\mathcal{X}_{x^{i_{1}}}^{k_{1}}\right] y_{k_{1}}+\sum_{k_{1}, k_{2}}\left[-\delta_{i_{1}}^{k_{1}} \mathcal{X}_{y}^{k_{2}}\right] y_{k_{1}} y_{k_{2}}\right)+ \\
& +\sum_{k_{1}}\left[-\mathcal{X}_{x^{i_{2}}}^{k_{1}}\right] y_{k_{1}, i_{1}}+\sum_{k_{1}}\left[-\mathcal{X}_{y}^{k_{1}}\right] y_{i_{2}} y_{i_{1}, k_{1}}
\end{aligned}
$$




$$
\begin{aligned}
= & \mathcal{Y}_{x^{i_{1}} x^{i_{2}}}+\sum_{k_{1}}\left[\delta_{i_{1}}^{k_{1}} \mathcal{Y}_{x^{i_{2} y}}-\mathcal{X}_{x^{i_{1}} x^{i_{2}}}^{k_{1}}\right] y_{k_{1}}+\sum_{k_{1}, k_{2}}\left[-\delta_{i_{1}}^{k_{1}} \mathcal{X}_{x^{i_{2}}}^{k_{2}}\right] y_{k_{1}} y_{k_{2}}+ \\
& +\mathcal{Y}_{x^{i_{1} y}} y_{i_{2}}+\sum_{k_{1}}\left[\delta_{i_{1}}^{k_{1}} \mathcal{Y}_{y y}-\mathcal{X}_{x^{i_{1} y}}^{k_{1}}\right] y_{k_{1}} y_{i_{2}}+\sum_{k_{1}, k_{2}}\left[-\delta_{i_{1}}^{k_{1}} \mathcal{X}_{y y}^{k_{2}}\right] y_{k_{1}} y_{k_{2}} y_{i_{2}}+ \\
& +\sum_{k_{1}}\left[\delta_{i_{1}}^{k_{1}} \mathcal{Y}_{y}-\mathcal{X}_{x^{i_{1}}}^{k_{1}}\right] y_{i_{2}, k_{1}}+\sum_{k_{1}, k_{2}}\left[-\delta_{i_{1}}^{k_{1}} \mathcal{X}_{y}^{k_{2}}\right] y_{k_{2}} y_{i_{2}, k_{1}}+\sum_{k_{1}, k_{2}}\left[-\delta_{i_{1}}^{k_{1}} \mathcal{X}_{y}^{k_{2}}\right] y_{k_{1}} y_{i_{2}, k_{2}}+ \\
& +\sum_{k_{1}}\left[-\mathcal{X}_{x^{i_{2}}}^{k_{1}}\right] y_{k_{1}, i_{1}}+\sum_{k_{1}}\left[-\mathcal{X}_{y}^{k_{1}}\right] y_{i_{2}} y_{i_{1}, k_{1}} .
\end{aligned}
$$

Some explanations are needed about the computation of the last two terms of line 11, i.e. about the passage from line 7 of (3.10) just above to line 11. We have to compute:

$$
\left(\sum_{k_{1}} y_{i_{2}, k_{1}} \frac{\partial}{\partial y_{k_{1}}}\right)\left(\sum_{k_{1}, k_{2}}\left[-\delta_{i_{1}}^{k_{1}} \mathcal{X}_{y}^{k_{2}}\right] y_{k_{1}} y_{k_{2}}\right) .
$$

This term is of the form

$$
\left(\sum_{k_{1}} A_{k_{1}} \frac{\partial}{\partial y_{k_{1}}}\right)\left(\sum_{k_{1}, k_{2}}\left[B_{k_{1}, k_{2}}\right] y_{k_{1}} y_{k_{2}}\right),
$$

where the terms $B_{k_{1}, k_{2}}$ are independent of the pure first jet variables $y_{x^{k}}$. By the rule of Leibniz for the differentiation of a product, we may write

$$
\begin{aligned}
& \left(\sum_{k_{1}} A_{k_{1}} \frac{\partial}{\partial y_{k_{1}}}\right)\left(\sum_{k_{1}, k_{2}}\left[B_{k_{1}, k_{2}}\right] y_{k_{1}} y_{k_{2}}\right)= \\
& =\sum_{k_{1}, k_{2}}\left[B_{k_{1}, k_{2}}\right] y_{k_{2}}\left(\sum_{k_{1}^{\prime}} A_{k_{1}^{\prime}} \frac{\partial}{\partial y_{k_{1}^{\prime}}}\left(y_{k_{1}}\right)\right)+\sum_{k_{1}, k_{2}}\left[B_{k_{1}, k_{2}}\right] y_{k_{1}}\left(\sum_{k_{2}^{\prime}} A_{k_{2}^{\prime}} \frac{\partial}{\partial y_{k_{2}^{\prime}}}\left(y_{k_{2}}\right)\right) \\
& =\sum_{k_{1}, k_{2}}\left[B_{k_{1}, k_{2}}\right] y_{k_{2}} A_{k_{1}}+\sum_{k_{1}, k_{2}}\left[B_{k_{1}, k_{2}}\right] y_{k_{1}} A_{k_{2}} .
\end{aligned}
$$

This is how we have written line 11 of (3.10).

Next, the first term $\mathcal{Y}_{x^{i_{1}} y} y_{i_{2}}$ in line 10 of (3.10) is not in a suitable shape. For reasons of harmony and coherence, we must insert it inside a sum of the form $\sum_{k_{1}}[\cdot] y_{k_{1}}$. Hence, using the Kronecker symbol, we transform:

$$
\mathcal{Y}_{x^{i_{1}} y} y_{i_{2}} \equiv \sum_{k_{1}}\left[\delta_{i_{2}}^{k_{1}} \mathcal{Y}_{x^{i_{1} y}}\right] y_{k_{1}}
$$

Also, we must "summify" the seven other terms, remaining in lines 10, 11 and 12 of (3.10). Sometimes, we use the symmetry $y_{i_{2}, k_{1}} \equiv y_{k_{1}, i_{2}}$ without mention. Similarly, we get:

$$
\begin{aligned}
\sum_{k_{1}}\left[\delta_{i_{1}}^{k_{1}} \mathcal{Y}_{y y}-\mathcal{X}_{x^{i_{1} y}}^{k_{1}}\right] y_{k_{1}} y_{i_{2}} & \equiv \sum_{k_{1}, k_{2}}\left[\delta_{i_{1}}^{k_{1}} \delta_{i_{2}}^{k_{2}} \mathcal{Y}_{y y}-\delta_{i_{2}}^{k_{2}} \mathcal{X}_{x^{i_{1}} y}^{k_{1}}\right] y_{k_{1}} y_{k_{2}}, \\
\sum_{k_{1}, k_{2}}\left[-\delta_{i_{1}}^{k_{1}} \mathcal{X}_{y y}^{k_{2}}\right] y_{k_{1}} y_{k_{2}} y_{i_{2}} & \equiv \sum_{k_{1}, k_{2}, k_{3}}\left[-\delta_{i_{1}}^{k_{1}} \delta_{i_{2}}^{k_{3}} \mathcal{X}_{y y}^{k_{2}}\right] y_{k_{1}} y_{k_{2}} y_{k_{3}}, \\
\sum_{k_{1}}\left[\delta_{i_{1}}^{k_{1}} \mathcal{Y}_{y}-\mathcal{X}_{x^{i_{1}}}^{k_{1}}\right] y_{k_{1}, i_{2}} & \equiv \sum_{k_{1}, k_{2}}\left[\delta_{i_{1}}^{k_{1}} \delta_{i_{2}}^{k_{2}} \mathcal{Y}_{y}-\delta_{i_{2}}^{k_{2}} \mathcal{X}_{x^{i_{1}}}^{k_{1}}\right] y_{k_{1}, k_{2}},
\end{aligned}
$$




$$
\begin{aligned}
\sum_{k_{1}, k_{2}}\left[-\delta_{i_{1}}^{k_{1}} \mathcal{X}_{y}^{k_{2}}\right] y_{k_{2}} y_{k_{1}, i_{2}} & =\sum_{k_{1}, k_{2}}\left[-\delta_{i_{1}}^{k_{2}} \mathcal{X}_{y}^{k_{1}}\right] y_{k_{1}} y_{k_{2}, i_{2}} \\
& \equiv \sum_{k_{1}, k_{2}, k_{3}}\left[-\delta_{i_{1}}^{k_{2}} \delta_{i_{2}}^{k_{3}} \mathcal{X}_{y}^{k_{1}}\right] y_{k_{1}} y_{k_{2}, k_{3}}, \\
\sum_{k_{1}, k_{2}}\left[-\delta_{i_{1}}^{k_{1}} \mathcal{X}_{y}^{k_{2}}\right] y_{k_{1}} y_{k_{2}, i_{2}} & \equiv \sum_{k_{1}, k_{2}, k_{3}}\left[-\delta_{i_{1}}^{k_{1}} \delta_{i_{2}}^{k_{3}} \mathcal{X}_{y}^{k_{2}}\right] y_{k_{1}} y_{k_{2}, k_{3}}, \\
\sum_{k_{1}}\left[-\mathcal{X}_{x^{i_{2}}}^{k_{1}}\right] y_{k_{1}, i_{1}} & \equiv \sum_{k_{1}, k_{2}}\left[-\delta_{i_{1}}^{k_{2}} \mathcal{X}_{x^{i_{2}}}^{k_{1}}\right] y_{k_{1}, k_{2}}, \\
\sum_{k_{1}}\left[-\mathcal{X}_{y}^{k_{1}}\right] y_{i_{2}} y_{k_{1}, i_{1}} & =\sum_{k_{2}}\left[-\mathcal{X}_{y}^{k_{2}}\right] y_{i_{2}} y_{k_{2}, i_{1}} \\
& \equiv \sum_{k_{1}, k_{2}, k_{3}}\left[-\delta_{i_{2}}^{k_{1}} \delta_{i_{1}}^{k_{3}} \mathcal{X}_{y}^{k_{2}}\right] y_{k_{1}} y_{k_{2}, k_{3}} .
\end{aligned}
$$

In the sequel, for products of Kronecker symbols, it will be convenient to adopt the following self-evident contracted notation:

$$
\delta_{i_{1}}^{k_{1}} \delta_{i_{2}}^{k_{2}} \equiv \delta_{i_{1}, i_{2}}^{k_{1}, k_{2}} ; \quad \text { generally : } \quad \delta_{i_{1}}^{k_{1}} \delta_{i_{2}}^{k_{2}} \cdots \delta_{i_{\lambda}}^{k_{\lambda}} \equiv \delta_{i_{1}, i_{2}, \cdots, i_{\lambda}}^{k_{1}, k_{2}, \cdots, k_{\lambda}} .
$$

Re-inserting plainly these eight summified terms (3.14), (3.15) in the last expression (3.10) of $\mathbf{Y}_{i_{1}, i_{2}}$ (lines 10, 11 and 12), we get:

$$
\begin{aligned}
& \mathbf{Y}_{i_{1}, i_{2}}=\underline{\mathcal{Y}_{x^{i_{1}} x^{i_{2}}}} \underline{1}+\sum_{k_{1}}\left[\delta_{i_{1}}^{k_{1}} \mathcal{Y}_{x^{i_{2} y}}-\mathcal{X}_{x^{i_{1}} x^{i_{2}}}^{k_{1}}\right] y_{k_{1}}+\sum_{2}\left[-\delta_{i_{1}, k_{2}}^{k_{1}} \mathcal{X}_{x^{i_{2} y}}^{k_{2}}\right] y_{k_{1}} y_{k_{2}}+ \\
& +\sum_{k_{1}}\left[\delta_{i_{2}}^{k_{1}} \mathcal{Y}_{x^{i_{1} y}}\right] y_{k_{1}}+\sum_{k_{1}, k_{2}}\left[\delta_{i_{1}, i_{2}}^{k_{1}, k_{2}} \mathcal{Y}_{y y}-\delta_{i_{2}}^{k_{2}} \mathcal{X}_{x^{i_{1}} y}^{k_{1}}\right] y_{k_{1}} y_{k_{2}}+ \\
& +\underbrace{}_{k_{1}, k_{2}, k_{3}}\left[-\delta_{i_{1}, i_{2}}^{k_{1}, k_{3}} \mathcal{X}_{y y}^{k_{2}}\right] y_{k_{1}} y_{k_{2}} y_{k_{3}}+\sum_{4}\left[\delta_{i_{1}, k_{2}}^{k_{1}, k_{2}} \mathcal{Y}_{y}-\delta_{i_{2}}^{k_{2}} \mathcal{X}_{x^{i_{1}}}^{k_{1}}\right] y_{k_{1}, k_{2}}+ \\
& +\sum_{k_{1}, k_{2}, k_{3}}\left[-\delta_{i_{1}, i_{2}}^{k_{2}, k_{3}} \mathcal{X}_{y}^{k_{1}}\right] y_{k_{1}} y_{k_{2}, k_{3}}+\sum_{6}\left[-\delta_{i_{1}, i_{2}}^{k_{1}, k_{3}} \mathcal{X}_{y}^{k_{2}}\right] y_{k_{1}, k_{2}, k_{3}, k_{3}}+ \\
& +\sum_{k_{1}, k_{2}}\left[-\delta_{i_{1}}^{k_{2}} \mathcal{X}_{x^{i_{2}}}^{k_{1}}\right] y_{k_{1}, k_{2}}+\sum_{5}\left[-\delta_{i_{2}, i_{1}}^{k_{1}, k_{3}, k_{3}} \mathcal{X}_{y}^{k_{2}}\right] y_{k_{1}} y_{k_{2}, k_{3}} .
\end{aligned}
$$

Next, we gather the underlined terms, ordering them according to their number. This yields 6 collections of sums of monomials in the pure jet variables:

$$
\begin{aligned}
\mathbf{Y}_{i_{1}, i_{2}}= & \mathcal{Y}_{x^{i_{1}} x^{i_{2}}}+\sum_{k_{1}}\left[\delta_{i_{1}}^{k_{1}} \mathcal{Y}_{x^{i_{2} y}}+\delta_{i_{2}}^{k_{1}} \mathcal{Y}_{x^{i_{1} y}}-\mathcal{X}_{x^{i_{1}} x^{i_{2}}}^{k_{1}}\right] y_{k_{1}}+ \\
& +\sum_{k_{1}, k_{2}}\left[\delta_{i_{1}, i_{2}}^{k_{1}, k_{2}} \mathcal{Y}_{y y}-\delta_{i_{1}}^{k_{1}} \mathcal{X}_{x^{i_{2} y}}^{k_{2}}-\delta_{i_{2}}^{k_{2}} \mathcal{X}_{x^{i_{1} y}}^{k_{1}}\right] y_{k_{1}} y_{k_{2}}+ \\
& +\sum_{k_{1}, k_{2}, k_{3}}\left[-\delta_{i_{1}, i_{2}}^{k_{1}, k_{3}} \mathcal{X}_{y y}^{k_{2}}\right] y_{k_{1}} y_{k_{2}} y_{k_{3}}+ \\
& +\sum_{k_{1}, k_{2}}\left[\delta_{i_{1}, i_{2}}^{k_{1}, k_{2}} \mathcal{Y}_{y}-\delta_{i_{2}}^{k_{2}} \mathcal{X}_{x^{i_{1}}}^{k_{1}}-\delta_{i_{1}}^{k_{2}} \mathcal{X}_{x^{i_{2}}}^{k_{1}}\right] y_{k_{1}, k_{2}}+ \\
& +\sum_{k_{1}, k_{2}, k_{3}}\left[-\delta_{i_{1}, i_{2}}^{k_{2}, k_{3}} \mathcal{X}_{y}^{k_{1}}-\delta_{i_{1}, i_{2}}^{k_{1}, k_{3}} \mathcal{X}_{y}^{k_{2}}-\delta_{i_{2}, i_{1}}^{k_{1}, k_{3}} \mathcal{X}_{y}^{k_{2}}\right] y_{k_{1}} y_{k_{2}, k_{3}} .
\end{aligned}
$$


To attain the real perfect harmony, this last expression has still to be worked out a little bit.

Lemma 3.19. The final expression of $\mathbf{Y}_{i_{1}, i_{2}}$ is as follows:

$$
\left\{\begin{aligned}
\mathbf{Y}_{i_{1}, i_{2}}= & \mathcal{Y}_{x^{i_{1}} x^{i_{2}}}+\sum_{k_{1}}\left[\delta_{i_{1}}^{k_{1}} \mathcal{Y}_{x^{i_{2}}}+\delta_{i_{2}}^{k_{1}} \mathcal{Y}_{x^{i_{1}} y}-\mathcal{X}_{x^{i_{1}} x^{i_{2}}}^{k_{1}}\right] y_{k_{1}}+ \\
& +\sum_{k_{1}, k_{2}}\left[\delta_{i_{1}, i_{2}}^{k_{1}, k_{2}} \mathcal{Y}_{y y}-\delta_{i_{1}}^{k_{1}} \mathcal{X}_{x^{i_{2} y}}^{k_{2}}-\delta_{i_{2}}^{k_{1}} \mathcal{X}_{x^{i_{1}} y}^{k_{2}}\right] y_{k_{1}} y_{k_{2}}+ \\
& +\sum_{k_{1}, k_{2}, k_{3}}\left[-\delta_{i_{1}, i_{2}}^{k_{1}, k_{2}} \mathcal{X}_{y y}^{k_{3}}\right] y_{k_{1}} y_{k_{2}} y_{k_{3}}+ \\
& +\sum_{k_{1}, k_{2}}\left[\delta_{i_{1}, i_{2}}^{k_{1}, k_{2}} \mathcal{Y}_{y}-\delta_{i_{1}}^{k_{1}} \mathcal{X}_{x^{i_{2}}}^{k_{2}}-\delta_{i_{2}}^{k_{1}} \mathcal{X}_{x^{i_{1}}}^{k_{2}}\right] y_{k_{1}, k_{2}}+ \\
& +\sum_{k_{1}, k_{2}, k_{3}}\left[-\delta_{i_{1}, i_{2}}^{k_{1}, k_{2}} \mathcal{X}_{y}^{k_{3}}-\delta_{i_{1}, i_{2}}^{k_{3}, k_{1}} \mathcal{X}_{y}^{k_{2}}-\delta_{i_{1}, i_{2}}^{k_{2}, k_{3}} \mathcal{X}_{y}^{k_{1}}\right] y_{k_{1}} y_{k_{2}, k_{3}} .
\end{aligned}\right.
$$

Proof. As promised, we explain every tiny detail.

The first lines of (3.18) and of (3.20) are exactly the same. For the transformations of terms in the second, in the third and in the fourth lines, we use the following device. Let $\Upsilon_{k_{1}, k_{2}}$ be an indexed quantity which is symmetric: $\Upsilon_{k_{1}, k_{2}}=\Upsilon_{k_{2}, k_{1}}$. Let $A_{k_{1}, k_{2}}$ be an arbitrary indexed quantity. Then obviously:

$$
\sum_{k_{1}, k_{2}} A_{k_{1}, k_{2}} \Upsilon_{k_{1}, k_{2}}=\sum_{k_{1}, k_{2}} A_{k_{2}, k_{1}} \Upsilon_{k_{1}, k_{2}}
$$

Similar relations hold with a quantity $\Upsilon_{i_{1}, i_{2}, \ldots, i_{\lambda}}$ which is symmetric with respect to its $\lambda$ indices. Consequently, in the second, in the third and in the fourth lines of (3.18), we may permute freely certain indices in some of the terms inside the brackets. This yields the passage from lines 2, 3 and 4 of (3.18) to lines 2, 3 and 4 of (3.20).

It remains to explain how we pass from the fifth (last) line of (3.18) to the fifth (last) line of (3.20). The bracket in the fifth line of (3.18) contains three terms: $\left[-T_{1}-T_{2}-T_{3}\right]$. The term $T_{3}$ involves the product $\delta_{i_{2}, i_{1}}^{k_{1}, k_{3}}$, which we rewrite as $\delta_{i_{1}, i_{2}}^{k_{3}, k_{1}}$, in order that $i_{1}$ appears before $i_{2}$. Then, we rewrite the three terms in the new order $\left[-T_{2}-T_{3}-T_{1}\right]$, which yields:

$$
\sum_{k_{1}, k_{2}, k_{3}}\left[-\delta_{i_{1}, i_{2}}^{k_{1}, k_{3}} \mathcal{X}_{y}^{k_{2}}-\delta_{i_{1}, i_{2}}^{k_{3}, k_{1}} \mathcal{X}_{y}^{k_{2}}-\delta_{i_{1}, i_{2}}^{k_{2}, k_{3}} \mathcal{X}_{y}^{k_{1}}\right] y_{k_{1}} y_{k_{2}, k_{3}}
$$

It remains to observe that we can permute $k_{2}$ and $k_{3}$ in the first term $-T_{2}$, which yields the last line of (3.20). The detailed proof is complete.

3.23. Final perfect expression of $\mathbf{Y}_{i_{1}, i_{2}, i_{3}}$. Thanks to similar (longer) computations, we have obtained an expression of $\mathbf{Y}_{i_{1}, i_{2}, i_{3}}$ which we consider to be in final harmonious 
shape. Without copying the intermediate steps, let us write down the result. The comments which are necessary to read it and to interpret it start just below.

$$
\begin{aligned}
& \mathbf{Y}_{i_{1}, i_{2}, i_{3}}=\mathcal{Y}_{x^{i_{1}} x^{i_{2}} x^{i_{3}}}+\sum_{k_{1}}\left[\delta_{i_{1}}^{k_{1}} \mathcal{Y}_{x^{i_{2}} x^{i_{3}} y}+\delta_{i_{2}}^{k_{1}} \mathcal{Y}_{x^{i_{1}} x^{i_{3} y}}+\delta_{i_{3}}^{k_{1}} \mathcal{Y}_{x^{i_{1}} x^{i_{2} y}}-\mathcal{X}_{x^{i_{1}} x^{i_{2}} x^{i_{3}}}^{k_{1}}\right] y_{k_{1}}+ \\
& +\sum_{k_{1}, k_{2}}\left[\delta_{i_{1}, i_{2}}^{k_{1}, k_{2}} \mathcal{Y}_{x^{i_{3}} y^{2}}+\delta_{i_{3}, i_{1}}^{k_{1}, k_{2}} \mathcal{Y}_{x^{i_{2}} y^{2}}+\delta_{i_{2}, i_{3}}^{k_{1}, k_{2}} \mathcal{Y}_{x^{i_{1}} y^{2}}\right. \\
& \left.-\delta_{i_{1}}^{k_{1}} \mathcal{X}_{x^{i_{2}} x^{i_{3} y}}^{k_{2}}-\delta_{i_{2}}^{k_{1}} \mathcal{X}_{x^{i_{1}} x^{i_{3}} y}^{k_{2}}-\delta_{i_{3}}^{k_{1}} \mathcal{X}_{x^{i_{1}} x^{i_{2} y}}^{k_{2}}\right] y_{k_{1}} y_{k_{2}}+ \\
& +\sum_{k_{1}, k_{2}, k_{3}}\left[\delta_{i_{1}, i_{2}, i_{3}}^{k_{1}, k_{2}, k_{3}} \mathcal{Y}_{y^{3}}-\delta_{i_{1}, i_{2}}^{k_{1}, k_{2}} \mathcal{X}_{x^{i_{3}} y^{2}}^{k_{3}}-\delta_{i_{1}, i_{3}}^{k_{1}, k_{2}} \mathcal{X}_{x^{i_{2}} y^{2}}^{k_{3}}-\delta_{i_{2}, i_{3}}^{k_{1}, k_{2}} \mathcal{X}_{x^{i_{1}} y^{2}}^{k_{3}}\right] y_{k_{1}} y_{k_{2}} y_{k_{3}}+ \\
& +\sum_{k_{1}, k_{2}, k_{3}, k_{4}}\left[-\delta_{i_{1}, i_{2}, i_{3}}^{k_{1}, k_{2}, k_{3}} \mathcal{X}_{y^{3}}^{k_{4}}\right] y_{k_{1}} y_{k_{2}} y_{k_{3}} y_{k_{4}}+ \\
& +\sum_{k_{1}, k_{2}}\left[\delta_{i_{1}, i_{2}}^{k_{1}, k_{2}} \mathcal{Y}_{x^{i_{3} y}}+\delta_{i_{3}, i_{1}}^{k_{1}, k_{2}} \mathcal{Y}_{x^{i_{2} y}}+\delta_{i_{2}, i_{3}}^{k_{1}, k_{2}} \mathcal{Y}_{x^{i_{1} y}}-\right. \\
& \left.-\delta_{i_{1}}^{k_{1}} \mathcal{X}_{x^{i_{2}} x^{i_{3}}}^{k_{2}}-\delta_{i_{2}}^{k_{1}} \mathcal{X}_{x^{i_{1}} x^{i_{3}}}^{k_{2}}-\delta_{i_{3}}^{k_{1}} \mathcal{X}_{x^{i_{1}} x^{i_{2}}}^{k_{2}}\right] y_{k_{1}, k_{2}}+ \\
& +\sum_{k_{1}, k_{2}, k_{3}}\left[\delta_{i_{1}, i_{2}, i_{3}}^{k_{1}, k_{2}, k_{3}} \mathcal{Y}_{y^{2}}+\delta_{i_{1}, i_{2}, i_{3}}^{k_{3}, k_{1}, k_{2}} \mathcal{Y}_{y^{2}}+\delta_{i_{1}, i_{2}, i_{3}}^{k_{2}, k_{3}, k_{1}} \mathcal{Y}_{y^{2}-}\right. \\
& -\delta_{i_{1}, i_{2}}^{k_{1}, k_{2}} \mathcal{X}_{x^{i_{3} y}}^{k_{3}}-\delta_{i_{1}, i_{2}}^{k_{3}, k_{1}} \mathcal{X}_{x^{i_{3}}}^{k_{2}}-\delta_{i_{1}, i_{2}}^{k_{2}, k_{3}} \mathcal{X}_{x^{i_{3}} y}^{k_{1}}- \\
& -\delta_{i_{1}, i_{3}}^{k_{1}, k_{2}} \mathcal{X}_{x^{i_{2}} y}^{k_{3}}-\delta_{i_{1}, i_{3}}^{k_{3}, k_{1}} \mathcal{X}_{x^{i_{2}} y}^{k_{2}}-\delta_{i_{1}, i_{3}}^{k_{2}, k_{3}} \mathcal{X}_{x^{i_{2}}}^{k_{1}}- \\
& \left.-\delta_{i_{2}, i_{3}}^{k_{1}, k_{2}} \mathcal{X}_{x^{i_{1} y}}^{k_{3}}-\delta_{i_{2}, i_{3}}^{k_{3}, k_{1}} \mathcal{X}_{x^{i_{1} y}}^{k_{2}}-\delta_{i_{2}, i_{3}}^{k_{2}, k_{3}} \mathcal{X}_{x^{i_{1} y}}^{k_{1}}\right] y_{k_{1}} y_{k_{2}, k_{3}}+ \\
& +\sum_{k_{1}, k_{2}, k_{3}, k_{4}}\left[-\delta_{i_{1}, i_{2}, i_{3}}^{k_{1}, k_{2}, k_{3}} \mathcal{X}_{y^{2}}^{k_{4}}-\delta_{i_{1}, i_{2}, i_{3}}^{k_{2}, k_{3}, k_{1}} \mathcal{X}_{y^{2}}^{k_{4}}-\delta_{i_{1}, i_{2}, i_{3}}^{k_{3}, k_{2}, k_{1}} \mathcal{X}_{y^{2}}^{k_{4}}-\right. \\
& \left.-\delta_{i_{1}, i_{2}, i_{3}}^{k_{3}, k_{4}, k_{1}} \mathcal{X}_{y^{2}}^{k_{2}}-\delta_{i_{1}, i_{2}, i_{3}}^{k_{3}, k_{1}, k_{4}} \mathcal{X}_{y^{2}}^{k_{2}}-\delta_{i_{1}, i_{2}, i_{3}}^{k_{1}, k_{3}, k_{4}} \mathcal{X}_{y^{2}}^{k_{2}}\right] y_{k_{1}} y_{k_{2}} y_{k_{3}, k_{4}}+ \\
& +\sum_{k_{1}, k_{2}, k_{3}, k_{4}}\left[-\delta_{i_{1}, i_{2}, i_{3}}^{k_{1}, k_{2}, k_{3}} \mathcal{X}_{y}^{k_{4}}-\delta_{i_{1}, i_{2}, i_{3}}^{k_{2}, k_{3}, k_{1}} \mathcal{X}_{y}^{k_{4}}-\delta_{i_{1}, i_{2}, i_{3}}^{k_{3}, k_{1}, k_{2}} \mathcal{X}_{y}^{k_{4}}\right] y_{k_{1}, k_{2}} y_{k_{3}, k_{4}}+ \\
& +\sum_{k_{1}, k_{2}, k_{3}}\left[\delta_{i_{1}, i_{2}, i_{3}}^{k_{1}, k_{2}, k_{3}} \mathcal{Y}_{y}-\delta_{i_{1}, i_{2}}^{k_{1}, k_{2}} \mathcal{X}_{x^{i_{3}}}^{k_{3}}-\delta_{i_{1}, i_{3}}^{k_{1}, k_{2}} \mathcal{X}_{x^{i_{2}}}^{k_{3}}-\delta_{i_{2}, i_{3}}^{k_{1}, k_{2}} \mathcal{X}_{x^{i_{1}}}^{k_{3}}\right] y_{k_{1}, k_{2}, k_{3}}+ \\
& +\sum_{k_{1}, k_{2}, k_{3}, k_{4}}\left[-\delta_{i_{1}, i_{2}, i_{3}}^{k_{1}, k_{2}, k_{3}} \mathcal{X}_{y}^{k_{4}}-\delta_{i_{1}, i_{2}, i_{3}}^{k_{4}, k_{1}, k_{2}} \mathcal{X}_{y}^{k_{3}}-\delta_{i_{1}, i_{2}, i_{3}}^{k_{3}, k_{4}, k_{1}} \mathcal{X}_{y}^{k_{2}}-\delta_{i_{1}, i_{2}, i_{3}}^{k_{2}, k_{3}, k_{4}} \mathcal{X}_{y}^{k_{1}}\right] y_{k_{1}} y_{k_{2}, k_{3}, k_{4}} .
\end{aligned}
$$

3.25. Comments, analysis and induction. First of all, by comparing this expression of $\mathbf{Y}_{i_{1}, i_{2}, i_{3}}$ with the expression (2.8) of $\mathbf{Y}_{3}$, we easily guess a part of the (inductional) dictionary beween the cases $n=1$ and the case $n \geqslant 1$. For instance, the three monomials $[\cdot]\left(y_{1}\right)^{3},[\cdot] y_{1} y_{2}$ and $[\cdot]\left(y_{1}\right)^{2} y_{2}$ in $\mathbf{Y}_{3}$ are replaced in $\mathbf{Y}_{i_{1}, i_{2}, i_{3}}$ by the following three sums:

$$
\sum_{k_{1}, k_{2}, k_{3}}[\cdot] y_{k_{1}} y_{k_{2}} y_{k_{3}}, \quad \sum_{k_{1}, k_{2}, k_{3}}[\cdot] y_{k_{1}} y_{k_{2}, k_{3}}, \quad \text { and } \quad \sum_{k_{1}, k_{2}, k_{3}, k_{4}}[\cdot] y_{k_{1}} y_{k_{2}} y_{k_{3}, k_{4}} \text {. }
$$

Similar formal correspondences may be observed for all the monomials of $\mathbf{Y}_{1}, \mathbf{Y}_{i_{1}}$, of $\mathbf{Y}_{2}, \mathbf{Y}_{i_{1}, i_{2}}$ and of $\mathbf{Y}_{3}, \mathbf{Y}_{i_{1}, i_{2}, i_{3}}$. Generally and inductively speaking, the monomial

$$
[\cdot]\left(y_{\lambda_{1}}\right)^{\mu_{1}} \cdots\left(y_{\lambda_{d}}\right)^{\mu_{d}}
$$

appearing in the expression (2.25) of $\mathbf{Y}_{\kappa}$ should be replaced by a certain multiple sum generalizing (3.26). However, it is necessary to think, to pause and to search for an appropriate formalism before writing down the desired multiple sum. 
The jet variable $y_{\lambda_{1}}$ should be replaced by a jet variable corresponding to a $\lambda_{1}$-th partial derivative, say $y_{k_{1}, \ldots, k_{\lambda_{1}}}$, where $k_{1}, \ldots, k_{\lambda_{1}}=1, \ldots, n$. For the moment, to simplify the discussion, we leave out the presence of a sum of the form $\sum_{k_{1}, \ldots, k_{\lambda_{1}}}$. The $\mu_{1}$-th power $\left(y_{\lambda_{1}}\right)^{\mu_{1}}$ should be replaced not by $\left(y_{k_{1}, \ldots, k_{\lambda_{1}}}\right)^{\mu_{1}}$, but by a product of $\mu_{1}$ different jet variables $y_{k_{1}, \ldots, k_{\lambda_{1}}}$ of length $\lambda$, with all indices $k_{\alpha}=1, \ldots, n$ being distinct. This rule may be confirmed by inspecting the expressions of $\mathbf{Y}_{i_{1}}$, of $\mathbf{Y}_{i_{1}, i_{2}}$ and of $\mathbf{Y}_{i_{1}, i_{2}, i_{3}}$. So $y_{k_{1}, \ldots, k_{\lambda_{1}}}$ should be developed as a product of the form

$$
y_{k_{1}, \ldots, k_{\lambda_{1}}} y_{k_{\lambda_{1}+1}, \ldots, k_{2 \lambda_{1}}} \cdots y_{k_{\left(\mu_{1}-1\right) \lambda_{1}+1}, \ldots, k_{\mu_{1} \lambda_{1}}}
$$

where

$$
k_{1}, \ldots, k_{\lambda_{1}}, \ldots, k_{\mu_{1} \lambda_{1}}=1, \ldots, n
$$

Consider now the product $\left(y_{\lambda_{1}}\right)^{\mu_{1}}\left(y_{\lambda_{2}}\right)^{\mu_{2}}$. How should it develope in the case of several independent variables? For instance, in the expression of $\mathbf{Y}_{i_{1}, i_{2}, i_{3}}$, we have developed the product $\left(y_{1}\right)^{2} y_{2}$ as $y_{k_{1}} y_{k_{2}} y_{k_{3}, k_{4}}$. Thus, a reasonable proposal of formalism would be that the product $\left(y_{\lambda_{1}}\right)^{\mu_{1}}\left(y_{\lambda_{2}}\right)^{\mu_{2}}$ should be developed as a product of the form

$$
\begin{aligned}
& y_{k_{1}, \ldots, k_{\lambda_{1}}} y_{k_{\lambda_{1}+1}, \ldots, k_{2 \lambda_{1}}} \cdots y_{k_{\left(\mu_{1}-1\right) \lambda_{1}+1}, \ldots, k_{\mu_{1} \lambda_{1}}} \\
& y_{k_{\mu_{1} \lambda_{1}+1}, \ldots, k_{\mu_{1} \lambda_{1}+\lambda_{2}}} \cdots y_{k_{\mu_{1} \lambda_{1}+\left(\mu_{2}-1\right) \lambda_{2}+1}, \ldots, k_{\mu_{1} \lambda_{1}+\mu_{2} \lambda_{2}}},
\end{aligned}
$$

where

$$
k_{1}, \ldots, k_{\lambda_{1}}, \ldots, k_{\mu_{1} \lambda_{1}}, \ldots, k_{\mu_{1} \lambda_{1}+\mu_{2} \lambda_{2}}=1, \ldots, n
$$

However, when trying to write down the development of the general monomial $\left(y_{\lambda_{1}}\right)^{\mu_{1}}\left(y_{\lambda_{2}}\right)^{\mu_{2}} \cdots\left(y_{\lambda_{d}}\right)^{\mu_{d}}$, we would obtain the complicated product

$$
\begin{aligned}
& y_{k_{1}, \ldots, k_{\lambda_{1}}} y_{k_{\lambda_{1}+1}, \ldots, k_{2 \lambda_{1}}} \cdots y_{k_{\left(\mu_{1}-1\right) \lambda_{1}+1}, \ldots, k_{\mu_{1} \lambda_{1}}}
\end{aligned}
$$

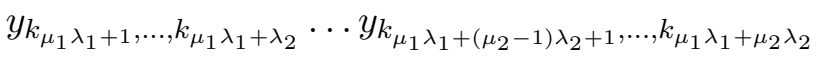

$$
\begin{aligned}
& y_{k_{\mu_{1} \lambda_{1}+\cdots+\mu_{d-1} \lambda_{d-1}+1}, \ldots, k_{\mu_{1} \lambda_{1}+\cdots+\mu_{d-1} \lambda_{d-1}+\lambda_{d}} \cdots} \cdots \\
& \cdots y_{k_{\mu_{1} \lambda_{1}+\cdots+\mu_{d-1} \lambda_{d-1}+\left(\mu_{d}-1\right) \lambda_{d}+1}, \ldots, k_{\mu_{1} \lambda_{1}+\cdots+\mu_{d} \lambda_{d}} .} .
\end{aligned}
$$

Essentially, this product is still readable. However, in it, some of the integers $k_{\alpha}$ have a too long index $\alpha$, often involving a sum. Such a length of $\alpha$ would be very inconvenient in writing down and in reading the general Kronecker symbols $\delta_{i_{1}, \ldots \ldots, i_{\lambda}}^{k_{\alpha_{1}, \ldots, k_{\lambda}}}$ which should appear in the final expression of $\mathbf{Y}_{i_{1}, \ldots, i_{\kappa}}$. One should read in advance Theorem 3.73 below to observe the presence of such multiple Kronecker symbols. Consequently, for $\alpha=1, \ldots, \mu_{1} \lambda_{1}, \ldots, \mu_{1} \lambda_{1}+\cdots+\mu_{d} \lambda_{d}$, we have to denote the indices $k_{\alpha}$ differently. 
Notational Convention 3.33. We denote d collection of $\mu_{d}$ groups of $\lambda_{d}$ (a priori distinct) integers $k_{\alpha}=1, \ldots, n$ by
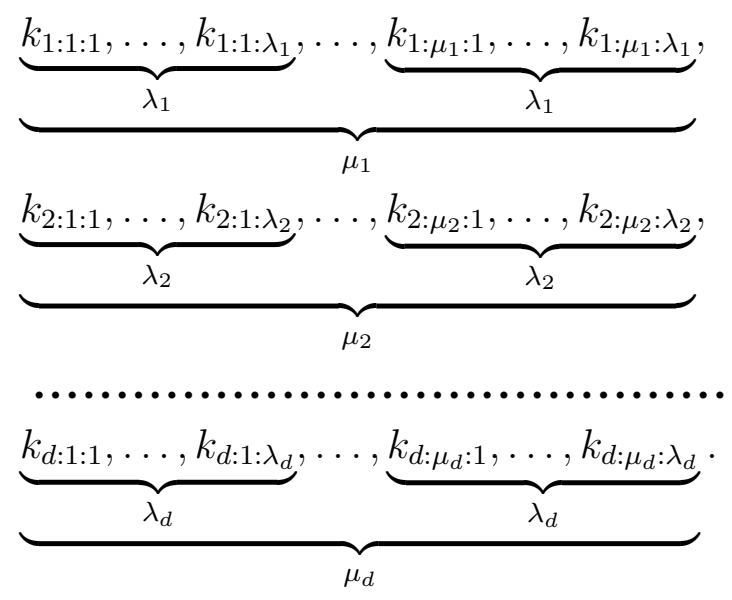

Correspondingly, we identify the set

$$
\left\{1, \ldots, \lambda_{1}, \ldots, \mu_{1} \lambda_{1}, \ldots \ldots, \mu_{1} \lambda_{1}+\mu_{2} \lambda_{2}, \ldots \ldots, \mu_{1} \lambda_{1}+\mu_{2} \lambda_{2}+\cdots+\mu_{d} \lambda_{d}\right\}
$$

of all integers $\alpha$ from 1 to $\mu_{1} \lambda_{1}+\mu_{2} \lambda_{2}+\cdots+\mu_{d} \lambda_{d}$ with the following specific set

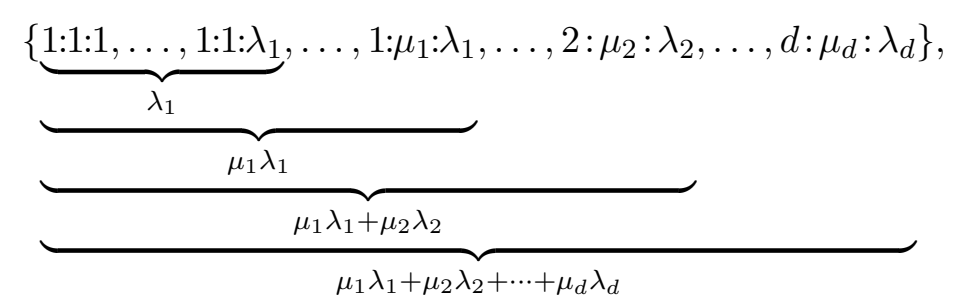

written in a lexicographic way which emphasizes clearly the subdivision in d collections of $\mu_{d}$ groups of $\lambda_{d}$ integers.

With this notation at hand, we see that the development, in several independent variables, of the general monomial $\left(y_{\lambda_{1}}\right)^{\mu_{1}} \cdots\left(y_{\lambda_{d}}\right)^{\mu_{d}}$, may be written as follows:

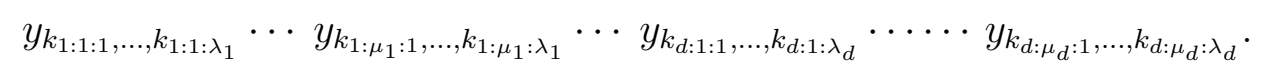

Formally speaking, this expression is better than (3.32). Using product symbols, we may even write it under the slightly more compact form

$$
\prod_{1 \leqslant \nu_{1} \leqslant \mu_{1}} y_{k_{1: \nu_{1}: 1,}, \ldots, k_{1: \nu_{1}: \lambda_{1}}} \cdots \prod_{1 \leqslant \nu_{d} \leqslant \mu_{d}} y_{k_{d: \nu_{d}: 1}, \ldots, k_{d: \nu_{d}: \lambda_{d}}} .
$$

Now that we have translated the monomial, we may add all the summation symbols: the general expression of $\mathbf{Y}_{\kappa}$ (which generalizes our three previous examples (3.26)) will be of the form:

$$
\begin{aligned}
& \mathbf{Y}_{\kappa}=\mathcal{Y}_{x^{i_{1} \ldots x^{i_{\kappa}}}}+\sum_{d=1}^{\kappa+1} \sum_{1 \leqslant \lambda_{1}<\cdots<\lambda_{d} \leqslant \kappa} \sum_{\mu_{1} \geqslant 1, \ldots, \mu_{d} \geqslant 1} \sum_{\mu_{1} \lambda_{1}+\cdots+\mu_{d} \lambda_{d} \leqslant \kappa+1}
\end{aligned}
$$

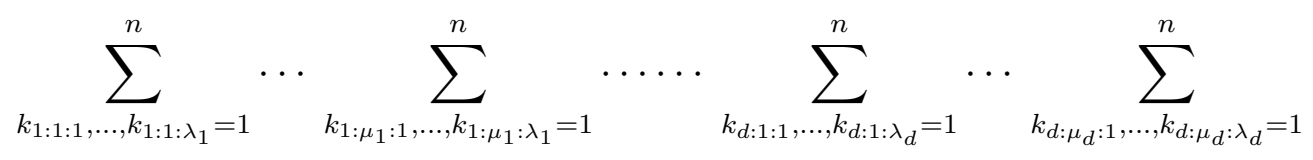

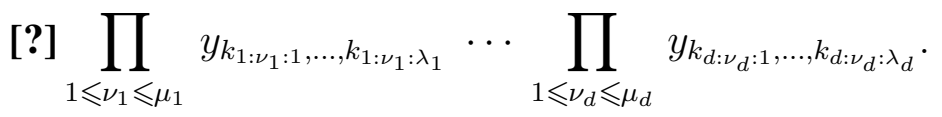

From now on, up to the end of the article, to be very precise, we will restitute the bounds $\sum_{k=1}^{n}$ of all the previously abbreviated sums $\sum_{k}$. This is justified by the fact that, 
since we shall deal in Section 5 below simultaneously with several independent variables $\left(x^{1}, \ldots, x^{n}\right)$ and with several dependent variables $\left(y^{1}, \ldots, y^{m}\right)$, we shall encounter sums $\sum_{l=1}^{m}$, not to be confused with sums $\sum_{k=1}^{n}$.

3.40. Combinatorics of the Kronecker symbols. Our next task is to determine what appears inside the brackets [?] of the above equation. We will treat this rather delicate question very progressively. Inductively, we have to guess how we may pass from the bracketed term of (2.25), namely from

$$
\begin{aligned}
& {\left[\frac{\kappa \cdots\left(\kappa-\mu_{1} \lambda_{1}-\cdots-\mu_{d} \lambda_{d}+1\right)}{\left(\lambda_{1} !\right)^{\mu_{1}} \mu_{1} ! \cdots\left(\lambda_{d} !\right)^{\mu_{d}} \mu_{d} !} \cdot \mathcal{Y}_{x^{\kappa-\mu_{1} \lambda_{1}-\cdots-\mu_{d} \lambda_{d}} y^{\mu_{1}+\cdots+\mu_{d}}-}-\right.} \\
& -\frac{\kappa \cdots\left(\kappa-\mu_{1} \lambda_{1}-\cdots-\mu_{d} \lambda_{d}+2\right)\left(\mu_{1} \lambda_{1}+\cdots+\mu_{d} \lambda_{d}\right)}{\left(\lambda_{1} !\right)^{\mu_{1}} \mu_{1} ! \cdots\left(\lambda_{d} !\right)^{\mu_{d}} \mu_{d} !} . \\
& \cdot \mathcal{X}_{\left.x^{\kappa-\mu_{1} \lambda_{1}-\cdots-\mu_{d} \lambda_{d}+1} y^{\mu_{1}+\cdots+\mu_{d}-1}\right]},
\end{aligned}
$$

to the corresponding (still unknown) bracketed term [?].

First of all, we examine the following term, extracted from the complete expression of $\mathbf{Y}_{i_{1}, i_{2}, i_{3}}$ (first line of (3.24)):

$$
\sum_{k_{1}=1}^{n}\left[\delta_{i_{1}}^{k_{1}} \mathcal{Y}_{x^{i_{2}} x_{3}{ }_{3}}+\delta_{i_{2}}^{k_{1}} \mathcal{Y}_{x^{i_{1}} x^{i_{3}} y}+\delta_{i_{3}}^{k_{1}} \mathcal{Y}_{x^{i_{1}} x^{i_{2} y}}-\mathcal{X}_{x^{i_{1}} x^{i_{2}} x^{i_{3}}}^{k_{1}}\right] y_{k_{1}}
$$

Here, the coefficient $\left[3 \mathcal{Y}_{x^{2} y}-\mathcal{X}_{x^{3}}\right]$ of the monomial $y_{1}$ in $\mathbf{Y}_{3}$ is replaced by the above bracketed terms.

Let us precisely analyze the combinatorics. Here, $\mathcal{X}_{x^{3}}$ is replaced by $\mathcal{X}_{x^{i_{1}} x^{i_{2}} x^{i_{3}}}^{k_{1}}$, where the lower indices $i_{1}, i_{2}, i_{3}$ come from $\mathbf{Y}_{i_{1}, i_{2}, i_{3}}$ and where the upper index $k_{1}$ is the summation index. Also, the integer 3 in $3 \mathcal{Y}_{x^{2} y}$ is replaced by a sum of exactly three terms, each involving a single Kronecker symbol $\delta_{i}^{k}$, in which the lower index is always an index $i=i_{1}, i_{2}, i_{3}$ and in which the upper index is always equal to the summation index $k_{1}$. By the way, more generally, we immediately observe that all the successive positive integers

$$
1,3,1,3,3,1,3,1,3,3,3,9,6,3,1,3,4
$$

appearing in the formula (2.8) for $\mathbf{Y}_{3}$ are replaced, in the formula (3.24) for $\mathbf{Y}_{i_{1}, i_{2}, i_{3}}$, by sums of exactly the same number of terms involving Kronecker symbols. This observation will be a precious guide. Finally, in the symbol $\delta_{i}^{k_{1}}$, if $i$ is chosen among the set $\left\{i_{1}, i_{2}, i_{3}\right\}$, for instance if $i=i_{1}$, it follows that the development of $\mathcal{Y}_{x^{2} y}$ necessarily involves the remaining indices, for instance $\mathcal{Y}_{x^{i_{2}} x^{i_{3}} y}$. Since there are three choices for $i=i_{1}, i_{2}, i_{3}$, we recover the number 3 .

Next, comparing $\left[\mathcal{Y}_{y y}-2 \mathcal{X}_{x y}\right]\left(y_{1}\right)^{2}$ with the term

$$
\sum_{k_{1}, k_{2}=1}^{n}\left[\delta_{i_{1}, i_{2}}^{k_{1}, k_{2}} \mathcal{Y}_{y y}-\delta_{i_{1}}^{k_{1}} \mathcal{X}_{x^{i_{2}} y}^{k_{1}}-\delta_{i_{2}}^{k_{1}} \mathcal{X}_{x^{i_{1}} y}^{k_{1}}\right] y_{k_{1}} y_{k_{2}}
$$

extracted from the complete expression of $\mathbf{Y}_{i_{1}, i_{2}}$ (second line of (3.18)), we learn and we guess that the number of Kronecker symbols before $\mathcal{Y}_{x^{\gamma} y^{\delta}}$ must be equal to the number of indices $k_{\alpha}$ minus $\gamma$. This rule is confirmed by examining the term (second and third line of (3.24))

$$
\begin{aligned}
\sum_{k_{1}, k_{2}}\left[\delta_{i_{1}, i_{2}}^{k_{1}, k_{2}} \mathcal{Y}_{x^{i_{3}} y^{2}}+\delta_{i_{3}, i_{1}}^{k_{1}, k_{2}} \mathcal{Y}_{x^{i_{2}} y^{2}}+\delta_{i_{2}, i_{3}}^{k_{1}, k_{2}} \mathcal{Y}_{x^{i_{1}} y^{2}}-\right. \\
\left.-\delta_{i_{1}}^{k_{1}} \mathcal{X}_{x^{i_{2} x^{i_{3} y}}}^{k_{2}}-\delta_{i_{2}}^{k_{1}} \mathcal{X}_{x^{i_{1}} x^{i_{3} y}}^{k_{2}}-\delta_{i_{3}}^{k_{1}} \mathcal{X}_{x^{i_{1}} x^{i_{2} y}}^{k_{2}}\right] y_{k_{1}} y_{k_{2}},
\end{aligned}
$$


developing $\left[3 \mathcal{Y}_{x y^{2}}-3 \mathcal{X}_{x^{2} y}\right]\left(y_{1}\right)^{2}$.

Also, we may examine the following term

$$
\begin{aligned}
\sum_{k_{1}, k_{2}=1}^{n}\left[\delta_{i_{1}, i_{2}}^{k_{1}, k_{2}} \mathcal{Y}_{x^{i_{3}} x^{i_{4}} y^{2}}+\delta_{i_{1}, i_{3}}^{k_{1}, k_{2}} \mathcal{Y}_{x^{i_{2}} x^{i_{4}} y^{2}}+\delta_{i_{1}, i_{4}}^{k_{1}, k_{2}} \mathcal{Y}_{x^{i_{2}} x^{i_{3}} y^{2}}+\right. \\
+\delta_{i_{2}, i_{3}}^{k_{1}, k_{2}} \mathcal{Y}_{x^{i_{1}} x^{i_{4}} y^{2}}+\delta_{i_{2}, i_{4}}^{k_{1}, k_{2}} \mathcal{Y}_{x^{i_{1}} x^{i_{3}} y^{2}}+\delta_{i_{3}, i_{4}}^{k_{1}, k_{2}} \mathcal{Y}_{x^{i_{1}} x^{i_{2}} y^{2}-} \\
\quad-\delta_{i_{1}}^{k_{1}} \mathcal{X}_{x^{i_{2}} x^{i_{3}} x^{i_{4}} y}^{k_{1}}-\delta_{i_{2}}^{k_{1}} \mathcal{X}_{x^{i_{1}} x^{i_{2}} x^{i_{3}} y}^{k_{1}}-\delta_{i_{3}}^{k_{1}} \mathcal{X}_{x^{i_{1}} x^{k_{2}} x^{i_{4}} y}^{k_{1}}- \\
\left.\quad-\delta_{i_{4}}^{k_{1}} \mathcal{X}_{x^{i_{1}} x^{i_{2}} x^{i_{3} y}}^{k_{1}}\right] y_{k_{1}} y_{k_{2}}
\end{aligned}
$$

extracted from $\mathbf{Y}_{i_{1}, i_{2}, i_{3}, i_{4}}$ and developing $\left[6 \mathcal{Y}_{x^{2} y^{2}}-4 \mathcal{X}_{x^{3} y}\right]\left(y_{1}\right)^{2}$. We would like to mention that we have not written the complete expression of $\mathbf{Y}_{i_{1}, i_{2}, i_{3}, i_{4}}$, because it would cover two and a half printed pages.

By inspecting the way how the indices are permuted in the multiple Kronecker symbols of the first two lines of this expression (3.46), we observe that the six terms correspond exactly to the six possible choices of two complementary ordered couples of integers in the set $\{1,2,3,4\}$, namely

$$
\begin{array}{lll}
\{1,2\} \cup\{3,4\}, & \{1,3\} \cup\{2,4\}, \quad\{1,4\} \cup\{2,3\}, \\
\{2,3\} \cup\{1,4\}, \quad\{2,4\} \cup\{1,3\}, \quad\{3,4\} \cup\{1,2\} .
\end{array}
$$

At this point, we start to devise the general combinatorics. Before proceeding further, we need some notation.

3.48. Permutation groups. For every $p \in \mathbb{N}$ with $p \geqslant 1$, we denote by $\mathfrak{S}_{p}$ the full permutation group of the set $\{1,2, \ldots, p-1, p\}$. Its cardinal equals $p$ !. The letters $\sigma$ and $\tau$ will be used to denote an element of $\mathfrak{S}_{p}$. If $p \geqslant 2$, and if $q \in \mathbb{N}$ satisfies $1 \leqslant q \leqslant p-1$, we denote by $\mathfrak{S}_{p}^{q}$ the subset of permutations $\sigma \in \mathfrak{S}_{p}$ satisfying the two collections of inequalities

$$
\sigma(1)<\sigma(2)<\cdots<\sigma(q) \quad \text { and } \quad \sigma(q+1)<\sigma(q+2)<\cdots<\sigma(p)
$$

The cardinal of $\mathfrak{S}_{p}^{q}$ equals $C_{p}^{q}=\frac{p !}{q !(p-q) !}$. 
Lemma 3.50. For $\kappa \geqslant 1$, the development of (2.20) to several independent variables $\left(x^{1}, \ldots, x^{n}\right)$ is:

$$
\begin{aligned}
& \mathbf{Y}_{i_{1}, i_{2}, \ldots, i_{\kappa}}=\mathcal{Y}_{x^{i_{1}} x^{i_{2} \ldots x^{i \kappa}}}+\sum_{k_{1}=1}^{n}\left[\sum_{\tau \in \mathfrak{S}_{\kappa}^{1}} \delta_{i_{\tau(1)}}^{k_{1}} \mathcal{Y}_{x^{i} \tau(2) \ldots x^{i} \tau(\kappa)}-\mathcal{X}_{x^{i_{1}} x^{i_{2} \ldots x^{i_{\kappa}}}}^{k_{1}}\right] y_{k_{1}}+
\end{aligned}
$$

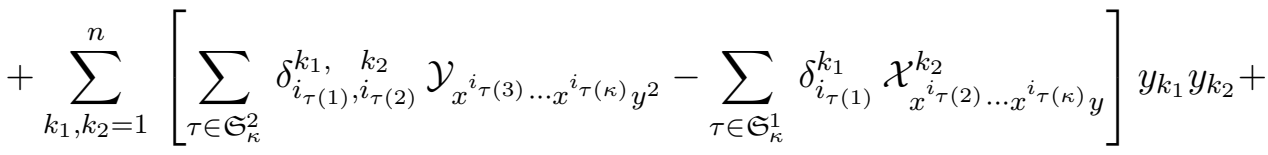

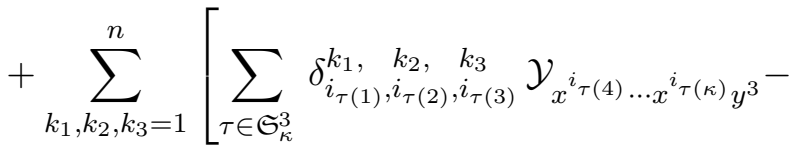

$$
\begin{aligned}
& \left.-\sum_{\tau \in \mathfrak{S}_{\kappa}^{2}} \delta_{i_{\tau(1), i} k_{\tau(2)}}^{k_{1}, \quad k_{2}} \mathcal{X}_{x^{i_{\tau}(3) \ldots x^{i} \tau(\kappa)} y^{2}}^{k_{3}}\right] y_{k_{1}} y_{k_{2}} y_{k_{3}}+ \\
& +\cdots+ \\
& +\sum_{k_{1}, \ldots, k_{\kappa}=1}^{n}\left[\delta_{i_{1}, \ldots, i_{\kappa}}^{k_{1}, \ldots, k_{\kappa}} \mathcal{Y}_{y^{\kappa}}-\sum_{\tau \in \mathfrak{S}_{\kappa}^{\kappa-1}} \delta_{i_{\tau(1)}, \ldots, i_{\tau(\kappa-1)}}^{k_{1}, \ldots \ldots, k_{\kappa-1}} \mathcal{X}_{x^{i_{\tau}(\kappa)} y^{\kappa-1}}^{k_{\kappa}}\right] y_{k_{1}} \cdots y_{k_{\kappa}}+ \\
& +\sum_{k_{1}, \ldots, k_{\kappa}, k_{\kappa+1}=1}^{n}\left[-\delta_{i_{1}, \ldots, i_{\kappa}}^{k_{1}, \ldots, k_{\kappa}} \mathcal{X}_{y^{\kappa}}^{k_{\kappa+1}}\right] y_{k_{1}} \cdots y_{k_{\kappa}} y_{k_{\kappa+1}}+\text { remainder }
\end{aligned}
$$

Here, the term remainder collects all remaining monomials in the pure jet variables $y_{k_{1}, \ldots, k_{\lambda}}$.

3.52. Continuation. Thus, we have devised how the part of $\mathbf{Y}_{i_{1}, \ldots, i_{\kappa}}$ which involves only the jet variables $y_{k_{\alpha}}$ must be written. To proceed further, we shall examine the following term, extracted from $\mathbf{Y}_{i_{1}, i_{2}, i_{3}}$ (lines 12 and 13 of (3.24))

$$
\begin{aligned}
\sum_{k_{1}, k_{2}, k_{3}, k_{4}}\left[-\delta_{i_{1}, i_{2}, i_{3}}^{k_{1}, k_{2}, k_{3}} \mathcal{X}_{y^{2}}^{k_{4}}-\delta_{i_{1}, i_{2}, i_{3}}^{k_{2}, k_{3}, k_{1}} \mathcal{X}_{y^{2}}^{k_{4}}-\delta_{i_{1}, i_{2}, i_{3}}^{k_{3}, k_{2}, k_{1}} \mathcal{X}_{y^{2}}^{k_{4}}-\right. \\
\left.\quad-\delta_{i_{1}, i_{2}, i_{3}}^{k_{3}, k_{4}, k_{1}} \mathcal{X}_{y^{2}}^{k_{2}}-\delta_{i_{1}, i_{2}, i_{3}}^{k_{3}, k_{1}, k_{4}} \mathcal{X}_{y^{2}}^{k_{2}}-\delta_{i_{1}, i_{2}, i_{3}}^{k_{1}, k_{3}, k_{4}} \mathcal{X}_{y^{2}}^{k_{2}}\right] y_{k_{1}} y_{k_{2}} y_{k_{3}, k_{4}},
\end{aligned}
$$

which developes the term $\left[-6 \mathcal{X}_{y^{2}}\right]\left(y_{1}\right)^{2} y_{2}$ of $\mathbf{Y}_{3}$ (third line of (2.8)). During the computation which led us to the final expression (3.24), we organized the formula in order that, in the six Kronecker symbols, the lower indices $i_{1}, i_{2}, i_{3}$ are all written in the same order. But then, what is the rule for the appearance of the four upper indices $k_{1}, k_{2}, k_{3}, k_{4}$ ?

In April 2001, we discovered the rule by inspecting both (3.53) and the following complicated term, extracted from the complete expression of $\mathbf{Y}_{i_{1}, i_{2}, i_{3}, i_{4}}$ written in one of our manuscripts:

$$
\begin{aligned}
& \sum_{k_{1}, k_{2}, k_{3}}\left[\delta_{i_{1}, i_{2}, i_{3}}^{k_{1}, k_{2}, k_{3}} \mathcal{Y}_{x^{i_{4}} y^{2}}+\delta_{i_{1}, i_{2}, i_{3}}^{k_{2}, k_{1}, k_{3}} \mathcal{Y}_{x^{i_{4}} y^{2}}+\delta_{i_{1}, i_{2}, i_{3}}^{k_{2}, k_{3}, k_{1}} \mathcal{Y}_{x^{i_{4}} y^{2}}+\right. \\
& +\delta_{i_{1}, i_{2}, i_{4}}^{k_{1}, k_{2}, k_{3}} \mathcal{Y}_{x^{i} 3} y^{2}+\delta_{i_{1}, i_{2}, i_{4}}^{k_{2}, k_{1}, k_{3}} \mathcal{Y}_{x^{i_{3}} y^{2}}+\delta_{i_{1}, i_{2}, i_{4}}^{k_{2}, k_{3}, k_{1}} \mathcal{Y}_{x^{i_{3}} y^{2}}+ \\
& +\delta_{i_{1}, i_{3}, i_{4}}^{k_{1}, k_{2}, k_{3}} \mathcal{Y}_{x^{i_{2}} y^{2}}+\delta_{i_{1}, i_{3}, i_{4}}^{k_{2}, k_{1}, k_{3}} \mathcal{Y}_{x^{i_{2}} y^{2}}+\delta_{i_{1}, i_{3}, i_{4}}^{k_{2}, k_{3}, k_{1}} \mathcal{Y}_{x^{i_{2}} y^{2}}+ \\
& +\delta_{i_{2}, i_{3}, i_{4}}^{k_{1}, k_{2}, k_{3}} \mathcal{Y}_{x^{i_{1}} y^{2}}+\delta_{i_{2}, i_{3}, i_{4}}^{k_{2}, k_{1}, k_{3}} \mathcal{Y}_{x^{i_{1}} y^{2}}+\delta_{i_{2}, i_{3}, i_{4}}^{k_{2}, k_{3}, k_{1}} \mathcal{Y}_{x^{i_{1}} y^{2}}- \\
& -\delta_{i_{1}, i_{2}}^{k_{1}, k_{2}} \mathcal{X}_{x^{i_{3} x^{i_{4} y}}}^{k_{3}}-\delta_{i_{1}, i_{2}}^{k_{2}, k_{1}} \mathcal{X}_{x^{i_{3} x^{i_{4}} y}}^{k_{3}}-\delta_{i_{1}, i_{2}}^{k_{2}, k_{3}} \mathcal{X}_{x^{i_{3} x^{i_{4}}}}^{k_{1}}-
\end{aligned}
$$




$$
\begin{aligned}
& -\delta_{i_{1}, i_{3}}^{k_{1}, k_{2}} \mathcal{X}_{x^{i_{2}} x^{i_{4} y}}^{k_{3}}-\delta_{i_{1}, i_{3}}^{k_{2}, k_{1}} \mathcal{X}_{x^{i_{2}} x^{i_{4}} y}^{k_{3}}-\delta_{i_{1}, i_{3}}^{k_{2}, k_{3}} \mathcal{X}_{x^{i_{2}} x^{i_{4}} y}^{k_{1}}- \\
& -\delta_{i_{1}, i_{4}}^{k_{1}, k_{2}} \mathcal{X}_{x^{i_{2}} x^{i_{3} y}}^{k_{3}}-\delta_{i_{1}, i_{4}}^{k_{2}, k_{1}} \mathcal{X}_{x^{i_{2}} x^{i_{3}} y}^{k_{3}}-\delta_{i_{1}, i_{4}}^{k_{2}, k_{3}} \mathcal{X}_{x^{i_{2}} x^{i_{3}} y}^{k_{1}}- \\
& -\delta_{i_{2}, i_{3}}^{k_{1}, k_{2}} \mathcal{X}_{x^{i_{1} x^{i_{4}} y}}^{k_{3}}-\delta_{i_{2}, i_{3}}^{k_{2}, k_{1}} \mathcal{X}_{x^{i_{1}} x^{i_{4} y}}^{k_{3}}-\delta_{i_{2}, i_{3}}^{k_{2}, k_{3}} \mathcal{X}_{x^{i_{1}} x^{i_{4}} y}^{k_{1}}- \\
& -\delta_{i_{2}, i_{4}}^{k_{1}, k_{2}} \mathcal{X}_{x^{i_{1}} x^{i_{3} y}}^{k_{3}}-\delta_{i_{2}, i_{4}}^{k_{2}, k_{1}} \mathcal{X}_{x^{i_{1}} x^{i_{3}} y}^{k_{3}}-\delta_{i_{2}, i_{4}}^{k_{2}, k_{3}} \mathcal{X}_{x^{i_{1}} x^{i_{3}} y}^{k_{1}}- \\
& \left.-\delta_{i_{3}, i_{4}}^{k_{1}, k_{2}} \mathcal{X}_{x^{i_{1}} x^{i_{2} y}}^{k_{3}}-\delta_{i_{3}, i_{4}}^{k_{2}, k_{1}} \mathcal{X}_{x^{i_{1}} x^{i_{2} y}}^{k_{3}}-\delta_{i_{3}, i_{4}}^{k_{2}, k_{3}} \mathcal{X}_{x^{i_{1}} x^{i_{2} y}}^{k_{1}}\right] y_{k_{1}} y_{k_{2}, k_{3}} .
\end{aligned}
$$

This sum developes the term $\left[12 \mathcal{Y}_{x y^{2}}-18 \mathcal{X}_{x^{2} y}\right] y_{1} y_{2}$ of $\mathbf{Y}_{3}$ (third line of (2.9)). Let us explain what are the formal rules.

In the bracketed terms of (3.53), there are no permutation of the indices $i_{1}, i_{2}, i_{3}$, but there is a certain unknown subset of all the permutations of the four indices $k_{1}, k_{2}, k_{3}, k_{4}$. In the bracketed terms of (3.54), two combinatorics are present:

- there are some permutations of the indices $i_{1}, i_{2}, i_{3}, i_{4}$ and

- there are some permutations of the indices $k_{1}, k_{2}, k_{3}$.

Here, the permutations of the indices $i_{1}, i_{2}, i_{3}, i_{4}$ are easily guessed, since they are the same as the permutations which were introduced in $\S 3.48$ above. Indeed, in the first four lines of (3.54), we see the four decompositions

$$
\left\{i_{1}, i_{2}, i_{3}\right\} \cup\left\{i_{4}\right\}, \quad\left\{i_{1}, i_{2}, i_{4}\right\} \cup\left\{i_{3}\right\}, \quad\left\{i_{1}, i_{3}, i_{4}\right\} \cup\left\{i_{2}\right\}, \quad\left\{i_{2}, i_{3}, i_{4}\right\} \cup\left\{i_{1}\right\},
$$

of the set $\left\{i_{1}, i_{2}, i_{3}, i_{4}\right\}$, and in the last six lines of (3.54), we see the six decompositions

$$
\begin{array}{lll}
\left\{i_{1}, i_{2}\right\} \cup\left\{i_{3}, i_{4}\right\}, & \left\{i_{1}, i_{3}\right\} \cup\left\{i_{2}, i_{4}\right\}, & \left\{i_{1}, i_{4}\right\} \cup\left\{i_{2}, i_{3}\right\}, \\
\left\{i_{2}, i_{3}\right\} \cup\left\{i_{1}, i_{4}\right\}, & \left\{i_{2}, i_{4}\right\} \cup\left\{i_{1}, i_{3}\right\}, & \left\{i_{3}, i_{4}\right\} \cup\left\{i_{1}, i_{2}\right\},
\end{array}
$$

so that (3.54) may be written under the form

$$
\sum_{k_{1}, k_{2}, k_{3}}\left[\sum_{\tau \in \mathfrak{S}_{4}^{3}} \sum_{\sigma \in ?} \delta_{i_{\tau(1)}, i_{\tau(2)}, i_{\tau(3)}}^{k_{\tau(1)}, k_{\tau(2)}, k_{\tau(3)}} \mathcal{Y}_{x^{i} \tau(4)} y^{2}-\sum_{\tau \in \mathfrak{S}_{4}^{2}} \sum_{\sigma \in \mathfrak{?}} \delta_{i_{\tau(1)}, i_{\tau(2)}}^{k_{\tau(1)}, k_{\tau(2)}} \mathcal{X}_{x^{i} \tau(3)}^{k_{\tau(3)} x^{i}(4) y}\right] y_{k_{1}} y_{k_{2}, k_{3}},
$$

where in the two above sums $\sum_{\sigma \in \text { ? }}$, the letter $\sigma$ denotes a permutation of the set $\{1,2,3\}$ and where the sign? refers to two (still unknown) subset of the full permutation group $\mathfrak{S}_{3}$. The only remaining question is to determine how the indices $k_{\alpha}$ are permuted in (3.53) and in (3.54).

The answer may be guessed by looking at the permutations of the set $\left\{k_{1}, k_{2}, k_{3}, k_{4}\right\}$ which stabilize the monomial $y_{k_{1}} y_{k_{2}} y_{k_{3}, k_{4}}$ in (3.53): we clearly have the following four symmetry relations between monomials:

$$
y_{k_{1}} y_{k_{2}} y_{k_{3}, k_{4}} \equiv y_{k_{2}} y_{k_{1}} y_{k_{3}, k_{4}} \equiv y_{k_{1}} y_{k_{2}} y_{k_{4}, k_{3}} \equiv y_{k_{2}} y_{k_{1}} y_{k_{4}, k_{3}}
$$

and nothing more. Then the number 6 of bracketed terms in (3.53) is exactly equal to the cardinal $24=4$ ! of the full permutation group of the set $\left\{k_{1}, k_{2}, k_{3}, k_{4}\right\}$ divided by the number 4 of these symmetry relations. The set of permutations $\sigma$ of $\{1,2,3,4\}$ satisfying these symmetry relations

$$
y_{k_{\sigma(1)}} y_{k_{\sigma(2)}} y_{k_{\sigma(3)}, k_{\sigma(4)}} \equiv y_{k_{1}} y_{k_{2}} y_{k_{3}, k_{4}}
$$

consitutes a subgroup of $\mathfrak{S}_{4}$ which we will denote by $\mathfrak{H}_{4}^{(2,1),(1,2)}$. Furthermore, the coset

$$
\mathfrak{F}_{4}^{(2,1),(1,2)}:=\mathfrak{S}_{4} / \mathfrak{H}_{4}^{(2,1),(1,2)}
$$


possesses the six representatives

$$
\begin{array}{lll}
\left(\begin{array}{llll}
1 & 2 & 3 & 4 \\
1 & 2 & 3 & 4
\end{array}\right), & \left(\begin{array}{llll}
1 & 2 & 3 & 4 \\
2 & 3 & 1 & 4
\end{array}\right), & \left(\begin{array}{llll}
1 & 2 & 3 & 4 \\
3 & 2 & 1 & 4
\end{array}\right), \\
\left(\begin{array}{llll}
1 & 2 & 3 & 4 \\
3 & 4 & 1 & 2
\end{array}\right), & \left(\begin{array}{llll}
1 & 2 & 3 & 4 \\
3 & 1 & 4 & 2
\end{array}\right), & \left(\begin{array}{llll}
1 & 2 & 3 & 4 \\
1 & 3 & 4 & 2
\end{array}\right),
\end{array}
$$

which exactly appear as the permutations of the upper indices of our example (3.53). Of course, the question arises whether the choice of such six representatives in the quotient $\mathfrak{S}_{4} / \mathfrak{H}_{4}^{(2,1),(1,2)}$ is legitimate.

Fortunately, we observe that after conjugation by any permutation $\sigma \in \mathfrak{H}_{4}^{(2,1),(1,2)}$, we do not perturb any of the six terms of (3.53), for instance the third term of (3.53) is not perturbed, as shown by the following computation

$$
\begin{aligned}
& \sum_{k_{1}, k_{2}, k_{3}, k_{4}}\left[-\delta_{i_{1},}^{k_{\sigma(3)}} \begin{array}{c}
k_{\sigma(2)}, k_{\sigma(1)} \\
i_{3}
\end{array} \mathcal{X}_{y^{2}}^{k_{\sigma(4)}}\right] y_{k_{1}} y_{k_{2}} y_{k_{3}, k_{4}}= \\
& =\sum_{k_{1}, k_{2}, k_{3}, k_{4}}\left[-\delta_{i_{1}, i_{2}, i_{3}}^{k_{3}, k_{2}, k_{1}} \mathcal{X}_{y^{2}}^{k_{\sigma(4)}}\right] y_{k_{\sigma^{-1}(1)}} y_{k_{\sigma^{-1}(2)}} y_{k_{\sigma^{-1}(3)}, k_{\sigma^{-1}(4)}} \\
& =\sum_{k_{1}, k_{2}, k_{3}, k_{4}}\left[-\delta_{i_{1}, i_{2}, i_{3}}^{k_{3}, k_{2}, k_{1}} \mathcal{X}_{y^{2}}^{k_{\sigma(4)}}\right] y_{k_{1}} y_{k_{2}} y_{k_{3}, k_{4}}
\end{aligned}
$$

thanks to the symmetry (3.59). Thus, as expected, the choice of 6 arbitrary representatives $\sigma \in \mathfrak{F}_{4}^{(2,1),(1,2)}$ in the bracketed terms of (3.53) is free. In conclusion, we have shown that (3.53) may be written under the form:

$$
\sum_{k_{1}, k_{2}, k_{3}, k_{4}}\left[-\sum_{\sigma \in \mathfrak{F}_{4}^{(2,1),(1,2)}} \delta_{i_{1}, i_{2}, \quad i_{3}}^{k_{\sigma(1)}, k_{\sigma(2)}, k_{\sigma(3)}} \mathcal{X}_{y^{2}}^{k_{\sigma(4)}}\right] y_{k_{1}} y_{k_{2}} y_{k_{3}, k_{4}},
$$

This rule is confirmed by inspecting (3.54) (as well as all the other terms of $\mathbf{Y}_{i_{1}, i_{2}, i_{3}}$ and of $\left.\mathbf{Y}_{i_{1}, i_{2}, i_{3}, i_{4}}\right)$. Indeed, the permutations $\sigma$ of the set $\left\{k_{1}, k_{2}, k_{3}\right\}$ which stabilize the monomial $y_{k_{1}} y_{k_{2}, k_{3}}$ consist just of the identity permutation and the transposition of $k_{2}$ and $k_{3}$. The coset $\mathfrak{S}_{3} / \mathfrak{H}_{3}^{(1,1),(1,2)}$ has the three representatives

$$
\left(\begin{array}{lll}
1 & 2 & 3 \\
1 & 2 & 3
\end{array}\right), \quad\left(\begin{array}{lll}
1 & 2 & 3 \\
2 & 1 & 3
\end{array}\right), \quad\left(\begin{array}{lll}
1 & 2 & 3 \\
2 & 3 & 1
\end{array}\right),
$$

which appear in the upper index position of each of the ten lines of (3.54). It follows that (3.54) may be written under the form

$$
\begin{aligned}
\sum_{k_{1}, k_{2}, k_{3}} & {\left[\sum_{\tau \in \mathfrak{S}_{4}^{3}} \sum_{\sigma \in \mathfrak{F}_{3}^{(1,1),(1,2)}} \delta_{i_{\tau(1)}, i_{\tau(2)}, i_{\tau(3)}}^{k_{\sigma(1)}, k_{\sigma(2)}, k_{\sigma(3)}} \mathcal{Y}_{x^{i_{\tau}(4)} y^{2}}-\right.} \\
& \left.-\sum_{\sigma \in \mathfrak{S}_{4}^{2}} \sum_{\tau \in \mathfrak{F}_{3}^{(1,1),(1,2)}} \delta_{i_{\tau(1)}, i_{\tau(2)}}^{k_{\sigma(1)}, k_{\sigma(2)}} \mathcal{X}_{x^{i_{\tau(3)}} x^{i_{\tau(4)}} y}^{k_{\sigma(3)}}\right] y_{k_{1}} y_{k_{2}, k_{3}} .
\end{aligned}
$$

3.66. General complete expression of $\mathbf{Y}_{i_{1}, \ldots, i_{\kappa}}$. As in the incomplete expression (3.39) of $\mathbf{Y}_{i_{1}, \ldots, i_{\kappa}}$, consider integers $1 \leqslant \lambda_{1}<\cdots<\lambda_{d} \leqslant \kappa$ and $\mu_{1} \geqslant 1, \ldots, \mu_{d} \geqslant 1$ satisfying $\mu_{1} \lambda_{1}+\cdots+\mu_{d} \lambda_{d} \leqslant \kappa+1$. By $\mathfrak{H}_{\mu_{1} \lambda_{1}+\cdots+\mathfrak{H}_{\mu_{d} \lambda_{d}}}$, we denote the subgroup of permutations $\tau \in$ 
$\mathfrak{S}_{\mu_{1} \lambda_{1}+\cdots+\mathfrak{H}_{\mu_{d} \lambda_{d}}}$ that leave unchanged the general monomial (3.38), namely that satisfy

$$
\begin{aligned}
& \prod_{1 \leqslant \nu_{1} \leqslant \mu_{1}} y_{k_{\sigma\left(1: \nu_{1}: 1\right)}, \ldots, k_{\sigma\left(1: \nu_{1}: \lambda_{1}\right)}} \cdots \prod_{1 \leqslant \nu_{d} \leqslant \mu_{d}} y_{k_{\sigma\left(d: \nu_{d}: 1\right)}, \ldots, k_{\sigma\left(d: \nu_{d}: \lambda_{d}\right)}}=
\end{aligned}
$$

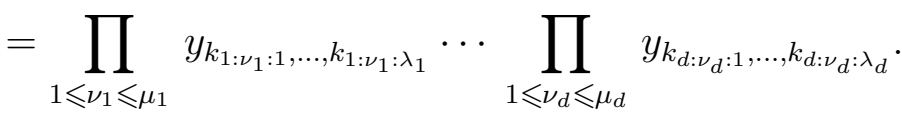

The structure of this group may be described as follows. For every $e=1, \ldots, d$, an arbitrary permutation $\sigma$ of the set

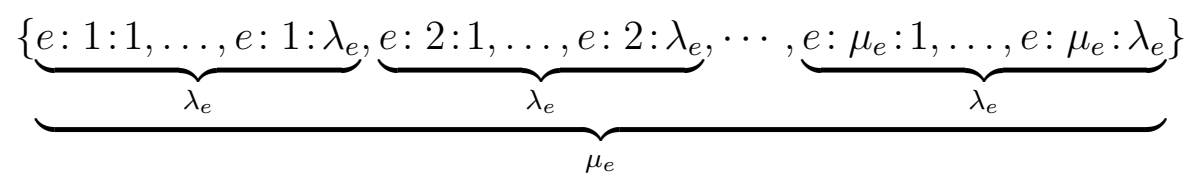

which leaves unchanged the monomial

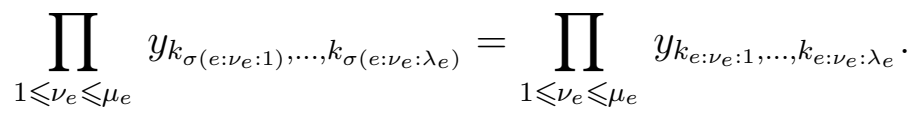

uniquely decomposes as the composition of

- $\mu_{e}$ arbitrary permutations of the $\mu_{e}$ groups of $\lambda_{e}$ integers $\left\{e: \nu_{e}: 1, \ldots, e: \nu_{e}: \lambda_{e}\right\}$ of total cardinal $\left(\lambda_{e} !\right)^{\mu_{e}}$;

- an arbitrary permutation between these $\mu_{e}$ groups, of total cardinal $\mu_{e}$ !.

Consequently

$$
\operatorname{Card}\left(\mathfrak{H}_{\mu_{1} \lambda_{1}+\cdots+\mu_{d} \lambda_{d}}^{\left(\mu_{1}, \lambda_{1}\right), \ldots,\left(\mu_{d}, \lambda_{d}\right)}\right)=\mu_{1} !\left(\lambda_{1} !\right)^{\mu_{1}} \cdots \mu_{d} !\left(\lambda_{d} !\right)^{\mu_{d}}
$$

Finally, define the coset

$$
\mathfrak{F}_{\mu_{1} \lambda_{1}+\cdots+\mu_{d} \lambda_{d}}^{\left(\mu_{1}, \lambda_{1}\right), \ldots,\left(\mu_{d}, \lambda_{d}\right)}:=\mathfrak{S}_{\mu_{1} \lambda_{1}+\cdots+\mu_{d} \lambda_{d}} / \mathfrak{H}_{\mu_{1} \lambda_{1}+\cdots+\mu_{d} \lambda_{d}}^{\left(\mu_{1}, \lambda_{1}\right), \ldots,\left(\mu_{d}, \lambda_{d}\right)}
$$

with

$$
\begin{aligned}
\operatorname{Card}\left(\mathfrak{F}_{\mu_{1} \lambda_{1}+\cdots+\mu_{d} \lambda_{d}}^{\left(\mu_{1}, \lambda_{1}\right), \ldots,\left(\mu_{d}, \lambda_{d}\right)}\right) & =\frac{\operatorname{Card}\left(\mathfrak{S}_{\mu_{1} \lambda_{1}+\cdots+\mu_{d} \lambda_{d}}\right)}{\operatorname{Card}\left(\mathfrak{H}_{\mu_{1} \lambda_{1}+\cdots+\mu_{d} \lambda_{d}}^{\left(\mu_{1}, \lambda_{1}\right), \ldots,\left(\mu_{d}, \lambda_{d}\right)}\right)} \\
& =\frac{\left(\mu_{1} \lambda_{1}+\cdots+\mu_{d} \lambda_{d}\right) !}{\mu_{1} !\left(\lambda_{1} !\right)^{\mu_{1}} \cdots \mu_{d} !\left(\lambda_{d} !\right)^{\mu_{d}}} .
\end{aligned}
$$

In conclusion, by means of this formalism, we may write down the complete generalization of Theorem 2.24 to several independent variables. 
Theorem 3.73. For every $\kappa \geqslant 1$ and for every choice of $\kappa$ indices $i_{1}, \ldots, i_{\kappa}$ in the set $\{1,2, \ldots, n\}$, the general expression of $\mathbf{Y}_{i_{1}, \ldots, i_{\kappa}}$ is as follows:

(3.74)

$$
\begin{aligned}
& \mathbf{Y}_{i_{1}, \ldots, i_{\kappa}}=\mathcal{Y}_{x^{i_{1} \ldots x^{i_{\kappa}}}}+\sum_{d=1}^{\kappa+1} \sum_{1 \leqslant \lambda_{1}<\cdots<\lambda_{d} \leqslant \kappa} \sum_{\mu_{1} \geqslant 1, \ldots, \mu_{d} \geqslant 1} \sum_{\mu_{1} \lambda_{1}+\cdots+\mu_{d} \lambda_{d} \leqslant \kappa+1}
\end{aligned}
$$

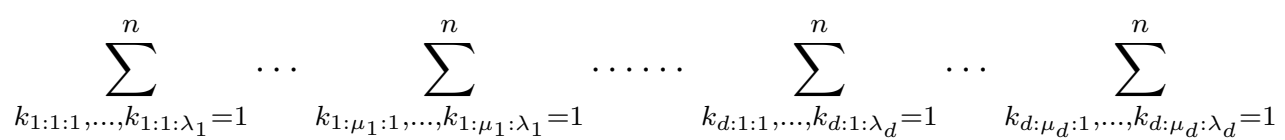

$$
\begin{aligned}
& \sum_{\sigma \in \mathfrak{F}_{\mu_{1} \lambda_{1}+\cdots+\mu_{d} \lambda_{d}}^{\left(\mu_{1}, \lambda_{1}\right), \ldots,\left(\mu_{d}, \lambda_{d}\right)}} \sum_{\tau \in \mathfrak{S}_{\kappa}^{\mu_{1} \lambda_{1}+\cdots+\mu_{d} \lambda_{d}}}
\end{aligned}
$$

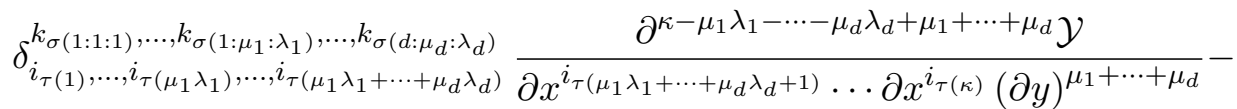

$$
\begin{aligned}
& -\sum_{\sigma \in \mathfrak{F}_{\mu_{1} \lambda_{1}+\cdots, \mu_{d} \lambda_{d}}^{\left.\left(\mu_{1}, \lambda_{1}\right), \ldots, \mu_{d}, \lambda_{d}\right)}} \sum_{\tau \in \mathfrak{S}_{\kappa}^{\mu_{1} \lambda_{1}+\cdots+\mu_{d} \lambda_{d}-1}}
\end{aligned}
$$

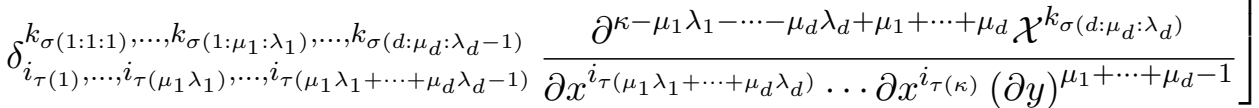

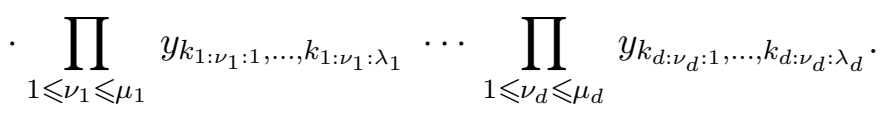

Since the fundamental monomials appearing in the last line of (3.74) just above are not independent of each other, this formula has still to be modified a little bit. We refer to Section 6 for details.

3.75. Deduction of a multivariate Faà di Bruno formula. Let $n \in \mathbb{N}$ with $n \geqslant 1$, let $x=\left(x^{1}, \ldots, x^{n}\right) \in \mathbb{K}^{n}$, let $g=g\left(x^{1}, \ldots, x^{n}\right)$ be a $\mathcal{C}^{\infty}$-smooth function from $\mathbb{K}^{n}$ to $\mathbb{K}$, let $y \in \mathbb{K}$ and let $f=f(y)$ be a $\mathcal{C}^{\infty}$ function from $\mathbb{K}$ to $\mathbb{K}$. The goal is to obtain an explicit formula for the partial derivatives of the composition $h:=f \circ g$, namely $h\left(x^{1}, \ldots, x^{n}\right):=f\left(g\left(x^{1}, \ldots, x^{n}\right)\right)$. For $\lambda \in \mathbb{N}$ with $\lambda \geqslant 1$ and for arbitrary indices $i_{1}, \ldots, i_{\lambda}=1, \ldots, n$, we shall abbreviate the partial derivative $\frac{\partial^{\lambda} g}{\partial x^{i_{1} \ldots \partial x^{i} \lambda}}$ by $g_{i_{1}, \ldots, i_{\lambda}}$ and similarly for $h_{i_{1}, \ldots, i_{\lambda}}$. The derivative $\frac{d^{\lambda} f}{d y^{\lambda}}$ will be abbreviated by $f_{\lambda}$.

Appying the chain rule, we may compute:

$$
\begin{aligned}
h_{i_{1}}= & f_{1}\left[g_{i_{1}}\right] \\
h_{i_{1}, i_{2}}= & f_{2}\left[g_{i_{1}} g_{i_{2}}\right]+f_{1}\left[g_{i_{1}, i_{2}}\right] \\
h_{i_{1}, i_{2}, i_{3}}= & f_{3}\left[g_{i_{1}} g_{i_{2}} g_{i_{3}}\right]+f_{2}\left[g_{i_{1}} g_{i_{2}, i_{3}}+g_{i_{2}} g_{i_{1}, i_{3}}+g_{i_{3}} g_{i_{1}, i_{2}}\right]+f_{1}\left[g_{i_{1}, i_{2}, i_{3}}\right], \\
h_{i_{1}, i_{2}, i_{3}, i_{4}}= & f_{4}\left[g_{i_{1}} g_{i_{2}} g_{i_{3}} g_{i_{4}}\right]+f_{3}\left[g_{i_{2}} g_{i_{3}} g_{i_{1}, i_{4}}+g_{i_{3}} g_{i_{1}} g_{i_{2}, i_{4}}+g_{i_{1}} g_{i_{2}} g_{i_{3}, i_{4}}+\right. \\
& \left.\quad+g_{i_{1}} g_{i_{4}} g_{i_{2}, i_{3}}+g_{i_{2}} g_{i_{4}} g_{i_{1}, i_{3}}+g_{i_{3}} g_{i_{4}} g_{i_{1}, i_{2}}\right]+ \\
& +f_{2}\left[g_{i_{1}, i_{2}} g_{i_{3}, i_{4}}+g_{i_{1}, i_{3}} g_{i_{2}, i_{4}}+g_{i_{1}, i_{4}} g_{i_{2}, i_{3}}\right]+ \\
& +f_{2}\left[g_{i_{1}} g_{i_{2}, i_{3}, i_{4}}+g_{i_{2}} g_{i_{1}, i_{3}, i_{4}}+g_{i_{3}} g_{i_{1}, i_{2}, i_{4}}+g_{i_{4}} g_{i_{1}, i_{2}, i_{3}}\right]+ \\
& +f_{1}\left[g_{i_{1}, i_{2}, i_{3}, i_{4}}\right] .
\end{aligned}
$$

Introducing the derivations

$$
\begin{aligned}
F_{i}^{2} & :=\sum_{k_{1}=1}^{n} g_{k_{1}, i} \frac{\partial}{\partial g_{k_{1}}}+g_{i}\left(f_{2} \frac{\partial}{\partial f_{1}}\right), \\
F_{i}^{3} & :=\sum_{k_{1}=1}^{n} g_{k_{1}, i} \frac{\partial}{\partial g_{k_{1}}}+\sum_{k_{1}, k_{2}=1}^{n} g_{k_{1}, k_{2}, i} \frac{\partial}{\partial g_{k_{1}, k_{2}}}+g_{i}\left(f_{2} \frac{\partial}{\partial f_{1}}+f_{3} \frac{\partial}{\partial f_{2}}\right),
\end{aligned}
$$




$$
\begin{aligned}
F_{i}^{\lambda}:=\sum_{k_{1}=1}^{n} g_{k_{1}, i} \frac{\partial}{\partial g_{k_{1}}}+\sum_{k_{1}, k_{2}=1}^{n} g_{k_{1}, k_{2}, i} \frac{\partial}{\partial g_{k_{1}, k_{2}}}+\cdots+ \\
+\sum_{k_{1}, \ldots, k_{\lambda-1}=1}^{n} g_{k_{1}, \ldots, k_{\lambda-1}, i} \frac{\partial}{\partial g_{k_{1}, \ldots, k_{\lambda-1}}}+ \\
+g_{i}\left(f_{2} \frac{\partial}{\partial f_{1}}+f_{3} \frac{\partial}{\partial f_{2}}+\cdots+f_{\lambda} \frac{\partial}{\partial f_{\lambda-1}}\right)
\end{aligned}
$$

we observe that the following induction relations hold:

$$
\begin{aligned}
& h_{i_{1}, i_{2}}=F_{i_{2}}^{2}\left(h_{i_{1}}\right), \\
& h_{i_{1}, i_{2}, i_{3}}=F_{i_{3}}^{3}\left(h_{i_{1}, i_{2}}\right) \text {, } \\
& \begin{array}{c}
\cdots \cdots \\
h_{i_{1}, i_{2}, \ldots, i_{\lambda}}
\end{array}=F_{i_{\lambda}}^{\lambda}\left(h_{i_{1}, i_{2}, \ldots, i_{\lambda-1}}\right) .
\end{aligned}
$$

To obtain the explicit version of the Faà di Bruno in the case of several variables $\left(x^{1}, \ldots, x^{n}\right)$ and one variable $y$, it suffices to extract from the expression of $\mathbf{Y}_{i_{1}, \ldots, i_{\kappa}}$ provided by Theorem 3.73 only the terms corresponding to $\mu_{1} \lambda_{1}+\cdots+\mu_{d} \lambda_{d}=\kappa$, dropping all the $\mathcal{X}$ terms. After some simplifications and after a translation by means of an elementary dictionary, we obtain a statement.

Theorem 3.79. For every integer $\kappa \geqslant 1$ and for every choice of indices $i_{1}, \ldots, i_{\kappa}$ in the set $\{1,2, \ldots, n\}$, the $\kappa$-th partial derivative of the composite function $h=h\left(x^{1}, \ldots, x^{n}\right)=$ $f\left(g\left(x^{1}, \ldots, x^{n}\right)\right)$ with respect to the variables $x^{i_{1}}, \ldots, x^{i_{\kappa}}$ may be expressed as an explicit polynomial depending on the derivatives of $f$, on the partial derivatives of $g$ and having integer coefficients:

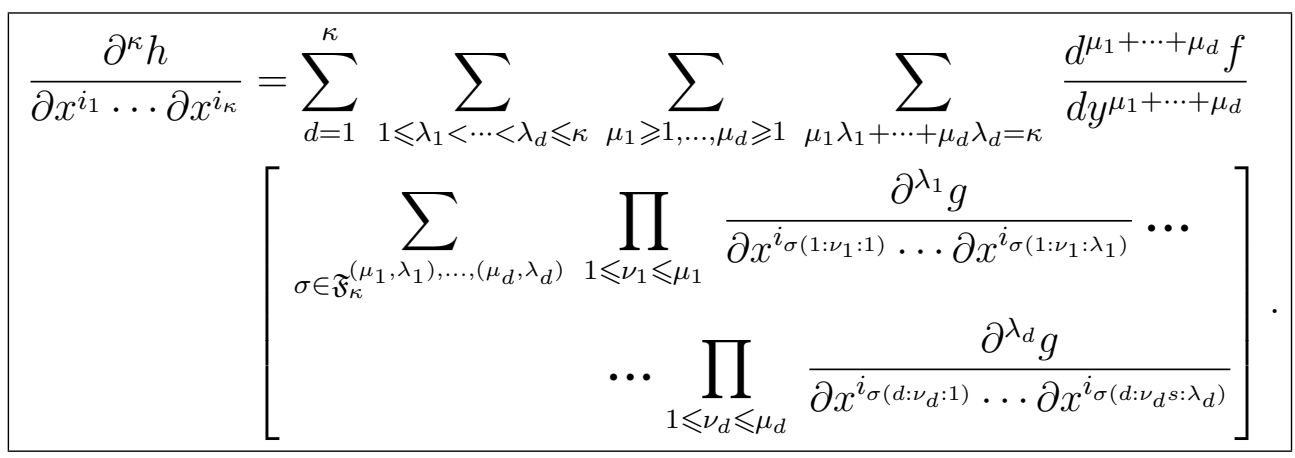

In this formula, the coset $\mathfrak{F}_{\kappa}^{\left(\mu_{1}, \lambda_{1}\right), \ldots,\left(\mu_{d}, \lambda_{d}\right)}$ was defined in equation (3.71); we have made the identification:

$$
\{1, \ldots, \kappa\} \equiv\left\{1: 1: 1, \ldots, 1: \mu_{1}: \lambda_{1}, \ldots, \ldots, d: 1: 1, \ldots, d: \mu_{d}: \lambda_{d}\right\} ;
$$

and also, for the sake of clarity, we have restituted the complete (not abbreviated) notation for the (partial) derivatives of $f$ and of $g$.

We refer to Section 6 for the final writing of the above formula (3.80).

\section{§4. ONE INDEPENDENT VARIABLE AND SEVERAL DEPENDENT VARIABLES}

4.1. Simplified adapted notations. Assume $n=1$ and $m \geqslant 1$, let $\kappa \in \mathbb{N}$ with $\kappa \geqslant 1$ and simply denote the jet variables by (instead of (1.2)):

$$
\left(x, y^{j}, y_{1}^{j}, y_{2}^{j}, \ldots, y_{\kappa}^{j}\right) \in \mathcal{J}_{1, m}^{\kappa} .
$$


Instead of (1.30), denote the $\kappa$-th prolongation of a vector field by:

$$
\left\{\begin{aligned}
\mathcal{L}^{(\kappa)}= & \mathcal{X} \frac{\partial}{\partial x}+\sum_{j=1}^{m} \mathcal{Y}^{j} \frac{\partial}{\partial y^{j}}+\sum_{j=1}^{m} \mathbf{Y}_{1}^{j} \frac{\partial}{\partial y_{1}^{j}}+\sum_{j=1}^{m} \mathbf{Y}_{2}^{j} \frac{\partial}{\partial y_{2}^{j}}+ \\
& +\cdots+\sum_{j=1}^{m} \mathbf{Y}_{\kappa}^{j} \frac{\partial}{\partial y_{\kappa}^{j}} .
\end{aligned}\right.
$$

The induction formulas are:

$$
\left\{\begin{aligned}
\mathbf{Y}_{1}^{j} & :=D^{1}\left(\mathcal{Y}^{j}\right)-D^{1}(\mathcal{X}) y_{1}^{j} \\
\mathbf{Y}_{2}^{j} & :=D^{2}\left(\mathbf{Y}_{1}^{j}\right)-D^{1}(\mathcal{X}) y_{2}^{j}, \\
\cdots & \cdots \cdots \\
\mathbf{Y}_{\lambda}^{j} & :=D^{\lambda}\left(\mathbf{Y}_{\lambda-1}^{j}\right)-D^{1}(\mathcal{X}) y_{\lambda}^{j},
\end{aligned}\right.
$$

where the total differentiation operators $D^{\lambda}$ are denoted by (instead of (1.22)):

$$
D^{\lambda}:=\frac{\partial}{\partial x}+\sum_{l=1}^{m} y_{1}^{l} \frac{\partial}{\partial y^{l}}+\sum_{l=1}^{m} y_{2}^{l} \frac{\partial}{\partial y_{1}^{l}}+\cdots+\sum_{l=1}^{m} y_{\lambda}^{l} \frac{\partial}{\partial y_{\lambda-1}^{l}}
$$

Applying the definitions in the first two lines of (4.4), we compute, we simplify and we organize the results in a harmonious way, using in an essential way the Kronecker symbol. Here, the computations are more elementary than the computations of $\mathbf{Y}_{i_{1}}$ and of $\mathbf{Y}_{i_{1}, i_{2}}$ achieved thoroughly in the previous Section 3, so that we do not provide a Latex track of the details. Firstly and secondly:

$$
\left\{\begin{aligned}
\mathbf{Y}_{1}^{j}= & \mathcal{Y}_{x}^{j}+\sum_{l_{1}=1}^{m}\left[\mathcal{Y}_{y^{l_{1}}}^{j}-\delta_{l_{1}}^{j} \mathcal{X}_{x}\right] y_{1}^{l_{1}}+\sum_{l_{1}, l_{2}=1}^{m}\left[-\delta_{l_{1}}^{j} \mathcal{X}_{y^{l_{2}}}\right] y_{1}^{l_{1}} y_{1}^{l_{2}} \\
\mathbf{Y}_{2}^{j}= & \mathcal{Y}_{x^{2}}^{j}+\sum_{l_{1}=1}^{m}\left[2 \mathcal{Y}_{x y^{l_{1}}}^{j}-\delta_{l_{1}}^{j} \mathcal{X}_{x^{2}}\right] y_{1}^{l_{1}}+\sum_{l_{1}, l_{2}=1}^{m}\left[\mathcal{Y}_{y^{l_{1}} y^{l_{2}}}^{j}-\delta_{l_{1}}^{j} 2 \mathcal{X}_{x y^{l_{2}}}\right] y_{1}^{l_{1}} y_{1}^{l_{2}+} \\
& +\sum_{l_{1}, l_{2}, l_{3}}^{m}\left[-\delta_{l_{1}}^{j} \mathcal{X}_{y^{l_{2}} y^{l_{3}}}\right] y_{1}^{l_{1}} y_{1}^{l_{2}} y_{1}^{l_{3}}+\sum_{l_{1}}\left[\mathcal{Y}_{y^{l_{1}}}^{j}-\delta_{l_{1}}^{j} 2 \mathcal{X}_{x}\right] y_{2}^{l_{1}}+ \\
& +\sum_{l_{1}, l_{2}=1}^{m}\left[-\delta_{l_{1}}^{j} \mathcal{X}_{y^{l_{2}}}-\delta_{l_{2}}^{j} 2 \mathcal{X}_{y^{l_{1}}}\right] y_{1}^{l_{1}} y_{2}^{l_{2}}
\end{aligned}\right.
$$

Thirdly:

$$
\begin{aligned}
\mathbf{Y}_{3}^{j}= & \mathcal{Y}_{x^{3}}^{j}+\sum_{l_{1}=1}^{m}\left[3 \mathcal{Y}_{x^{2} y^{l_{1}}}^{j}-\delta_{l_{1}}^{j} \mathcal{X}_{x^{3}}\right] y_{1}^{l_{1}}+\sum_{l_{1}, l_{2}=1}^{m}\left[3 \mathcal{Y}_{x y^{l_{1}} y^{l_{2}}}^{j}-\delta_{l_{1}}^{j} 3 \mathcal{X}_{x^{2} y^{l_{2}}}\right] y_{1}^{l_{1}} y_{1}^{l_{2}}+ \\
& +\sum_{l_{1}, l_{2}, l_{3}}\left[\mathcal{Y}_{y^{l_{1}} y^{l_{2}} y^{l_{3}}}^{j}-\delta_{l_{1}}^{j} 3 \mathcal{X}_{x y_{2} y_{2} y_{3}}\right] y_{1}^{l_{1}} y_{1}^{l_{2}} y_{1}^{l_{3}}+ \\
& +\sum_{l_{1}, l_{2}, l_{3}, l_{4}}\left[-\delta_{l_{1}}^{j} \mathcal{X}_{y^{l_{2}} y^{l_{3}} y^{l_{4}}}\right] y_{1}^{l_{1}} y_{1}^{l_{2}} y_{1}^{l_{3}} y_{1}^{l_{4}}+\sum_{l_{1}=1}^{m}\left[3 \mathcal{Y}_{x y_{1}^{l_{1}}}^{j}-\delta_{l_{1}}^{j} 3 \mathcal{X}_{x^{2}}\right] y_{2}^{l_{1}}+
\end{aligned}
$$




$$
\begin{aligned}
& +\sum_{l_{1}, l_{2}=1}^{m}\left[3 \mathcal{Y}_{y^{l_{1}} y^{l_{2}}}^{j}-\delta_{l_{1}}^{j} 3 \mathcal{X}_{x y^{l_{2}}}-\delta_{l_{2}}^{j} 6 \mathcal{X}_{x y^{l_{1}}}\right] y_{1}^{l_{1}} y_{2}^{l_{2}}+ \\
& +\sum_{l_{1}, l_{2}, l_{3}=1}^{m}\left[-\delta_{l_{1}}^{j} 3 \mathcal{X}_{y^{l_{2}} y^{l_{3}}}-\delta_{l_{3}}^{j} 3 \mathcal{X}_{y^{l_{1}} y^{l_{2}}}\right] y_{1}^{l_{1}} y_{1}^{l_{2}} y_{2}^{l_{3}}+\sum_{l_{1}, l_{2}=1}^{m}\left[-\delta_{l_{3}}^{j} 3 \mathcal{X}_{y^{l_{2}}}\right] y_{2}^{l_{1}} y_{2}^{l_{2}}+ \\
& +\sum_{l_{1}=1}^{m}\left[\mathcal{Y}_{y^{l_{1}}}^{j}-\delta_{l_{1}}^{j} 3 \mathcal{X}_{x}\right] y_{3}^{l_{1}}+\sum_{l_{1}, l_{2}=1}^{m}\left[-\delta_{l_{1}}^{j} \mathcal{X}_{y^{l_{2}}}-\delta_{l_{2}}^{j} 3 \mathcal{X}_{y^{l_{1}}}\right] y_{1}^{l_{1}} y_{3}^{l_{2}}
\end{aligned}
$$

Fourthly:

$$
\begin{aligned}
& \mathbf{Y}_{4}^{j}=\mathcal{Y}_{x^{4}}^{j}+\sum_{l_{1}=1}^{m}\left[4 \mathcal{Y}_{x^{3} y^{l_{1}}}^{j}-\delta_{l_{1}}^{j} \mathcal{X}_{x^{4}}\right] y_{1}^{l_{1}}+\sum_{l_{1}, l_{2}=1}^{m}\left[6 \mathcal{Y}_{x^{2} y^{l_{1}} y^{l_{2}}}^{j}-\delta_{l_{1}}^{j} 4 \mathcal{X}_{x^{3} y^{l_{2}}}\right] y_{1}^{l_{1}} y_{1}^{l_{2}}+ \\
& +\sum_{l_{1}, l_{2}, l_{3}=1}^{m}\left[4 \mathcal{Y}_{x y^{l_{1}} y^{l_{2}} y^{l_{3}}}^{j}-\delta_{l_{1}}^{j} 6 \mathcal{X}_{x^{2} y^{l_{2}} y^{l_{3}}}\right] y_{1}^{l_{1}} y_{1}^{l_{2}} y_{1}^{l_{3}}+ \\
& +\sum_{l_{1}, l_{2}, l_{3}, l_{4}=1}^{m}\left[\mathcal{Y}_{x y^{l_{1}} y^{l_{2}} y^{l_{3}} y^{l_{4}}}-\delta_{l_{1}}^{j} 4 \mathcal{X}_{\left.x y^{l_{2}} y^{l_{3}} y^{l_{4}}\right]}\right] y_{1}^{l_{1}} y_{1}^{l_{2}} y_{1}^{l_{3}} y_{1}^{l_{4}}+ \\
& +\sum_{l_{1}, l_{2}, l_{3}, l_{4}, l_{5}=1}^{m}\left[-\delta_{l_{1}}^{j} \mathcal{X}_{y^{l_{2}} y^{l_{3}} y^{l_{4}} y^{l_{5}}}\right] y_{1}^{l_{1}} y_{1}^{l_{2}} y_{1}^{l_{3}} y_{1}^{l_{4}} y_{1}^{l_{5}}+\sum_{l_{1}=1}^{m}\left[6 \mathcal{Y}_{x^{2} y^{l_{1}}}^{j}-\delta_{l_{1}}^{j} 4 \mathcal{X}_{x^{3}}\right] y_{2}^{l_{1}}+ \\
& +\sum_{l_{1}, l_{2}=1}^{m}\left[12 \mathcal{Y}_{x y^{l_{1}} y^{l_{2}}}^{j}-\delta_{l_{1}}^{j} 6 \mathcal{X}_{x^{2} y^{l_{2}}}-\delta_{l_{2}}^{j} 12 \mathcal{X}_{x^{2} y^{l_{1}}}\right] y_{1}^{l_{1}} y_{2}^{l_{2}}+ \\
& +\sum_{l_{1}, l_{2}, l_{3}=1}^{m}\left[6 \mathcal{Y}_{y^{l_{1}} y^{l_{2}} y^{l_{3}}}^{j}-\delta_{l_{1}}^{j} 12 \mathcal{X}_{x y_{2} y^{l_{3}}}-\delta_{l_{3}}^{j} 12 \mathcal{X}_{x y^{l_{1}} y^{l_{2}}}\right] y_{1}^{l_{1}} y_{1}^{l_{2}} y_{2}^{l_{3}}+ \\
& +\sum_{l_{1}, l_{2}, l_{3}, l_{4}=1}^{m}\left[-\delta_{l_{1}}^{j} 6 \mathcal{X}_{y^{l_{2}} y^{l_{3}} y^{l_{4}}}-\delta_{l_{4}}^{j} 4 \mathcal{X}_{y^{l_{1}} y^{l_{2}} y^{l_{3}}}\right] y_{1}^{l_{1}} y_{1}^{l_{2}} y_{1}^{l_{3}} y_{2}^{l_{4}}+ \\
& +\sum_{l_{1}, l_{2}=1}^{m}\left[3 \mathcal{Y}_{y^{l_{1}} y^{l_{2}}}^{j}-\delta_{l_{1}}^{j} 12 \mathcal{X}_{x y^{l_{2}}}\right] y_{2}^{l_{1}} y_{2}^{l_{2}}+ \\
& +\sum_{l_{1}, l_{2}, l_{3}=1}^{m}\left[-\delta_{l_{1}}^{j} 3 \mathcal{X}_{y^{l_{2}} y^{l_{3}}}-\delta_{l_{2}}^{j} 12 \mathcal{X}_{y^{l_{1}} y^{l_{3}}}\right] y_{1}^{l_{1}} y_{2}^{l_{2}} y_{2}^{l_{3}}+\sum_{l_{1}=1}^{m}\left[4 \mathcal{Y}_{x y^{l_{1}}}^{j}-\delta_{l_{1}}^{j} 6 \mathcal{X}_{x^{2}}\right] y_{3}^{l_{1}}+ \\
& +\sum_{l_{1}, l_{2}=1}^{m}\left[4 \mathcal{Y}_{y^{l_{1} y^{l_{2}}}}^{j}-\delta_{l_{1}}^{j} 4 \mathcal{X}_{x y^{l_{2}}}-\delta_{l_{2}}^{j} 12 \mathcal{X}_{x y^{l_{1}}}\right] y_{1}^{l_{1}} y_{3}^{l_{2}}+ \\
& +\sum_{l_{1}, l_{2}, l_{3}=1}^{m}\left[-\delta_{l_{1}}^{j} 4 \mathcal{X}_{y^{l_{2}} y^{l_{3}}}-\delta_{l_{3}}^{j} 6 \mathcal{X}_{y^{l_{1}} y^{l_{2}}}\right] y_{1}^{l_{1}} y_{1}^{l_{2}} y_{3}^{l_{3}}+ \\
& +\sum_{l_{1}, l_{2}=1}^{m}\left[-\delta_{l_{1}}^{j} 4 \mathcal{X}_{y^{l_{2}}}-\delta_{l_{2}}^{j} 6 \mathcal{X}_{y^{l_{1}}}\right] y_{2}^{l_{1}} y_{3}^{l_{2}}+ \\
& +\sum_{l_{1}=1}^{m}\left[\mathcal{Y}_{y^{l_{1}}}^{j}-\delta_{l_{1}}^{j} 4 \mathcal{X}_{x}\right] y_{4}^{l_{1}}+\sum_{l_{1}, l_{2}=1}^{m}\left[-\delta_{l_{1}}^{j} \mathcal{X}_{y^{l_{2}}}-\delta_{l_{2}}^{j} 4 \mathcal{X}_{y^{l_{1}}}\right] y_{1}^{l_{1}} y_{4}^{l_{2}}
\end{aligned}
$$

4.9. Inductive elaboration of the general formula. Now we compare the formula (2.9) for $\mathbf{Y}_{4}$ with the above formula (4.8) for $\mathbf{Y}_{4}^{j}$. The goal is to find the rules of transformation and of development by inspecting several instances, in order to devise how to transform and to develope the formula (2.25) to several dependent variables. 
First of all, we have to develope the general monomial $\left(y_{\lambda_{1}}\right)^{\mu_{1}} \cdots\left(y_{\lambda_{d}}\right)^{\mu_{d}}$. In every monomial present in the expressions of $\mathbf{Y}_{1}^{j}$, of $\mathbf{Y}_{2}^{j}$, of $\mathbf{Y}_{3}^{j}$ and of $\mathbf{Y}_{4}^{j}$ above, we see that the number $\alpha$ of indices $l_{\beta}$ appearing in all the sums $\sum_{l_{1}, \ldots, l_{\alpha}=1}^{m}$ is exactly equal to $\mu_{1}+$ $\cdots+\mu_{d}$. To denote these $\mu_{1}+\cdots+\mu_{d}$ indices $l_{\beta}$, we shall use the notation:

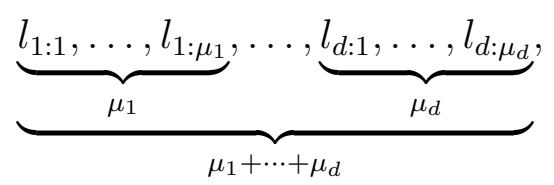

inspired by Convention 3.33. With such a choice of notation, we may avoid long subscripts in the indices $l_{\beta}$, like $l_{\mu_{1}+\cdots+\mu_{d}}$. It follows that the development of the general monomial $\left(y_{\lambda_{1}}\right)^{\mu_{1}} \cdots\left(y_{\lambda_{d}}\right)^{\mu_{d}}$ to several dependent variables yields $m^{\mu_{1}+\cdots+\mu_{d}}$ possible choices:

$$
\prod_{1 \leqslant \nu_{1} \leqslant \mu_{1}} y_{\lambda_{1}}^{l_{1: \nu_{1}}} \ldots \cdots \prod_{1 \leqslant \nu_{d} \leqslant \mu_{d}} y_{\lambda_{d}}^{l_{d: \nu_{d}}}
$$

where the indices $l_{1: 1}, \ldots, l_{1: \mu_{1}}, \ldots, l_{d: 1}, \ldots, l_{d: \mu_{d}}$ take their values in the set $\{1,2, \ldots, m\}$. Consequently, the general expression of $\mathbf{Y}_{\kappa}^{j}$ must be of the form:

$$
\begin{aligned}
& \mathbf{Y}_{\kappa}^{j}=\mathcal{Y}_{x^{\kappa}}^{j}+\sum_{d=1}^{\kappa+1} \sum_{1 \leqslant \lambda_{1}<\cdots<\lambda_{d} \leqslant \kappa} \sum_{\mu_{1} \geqslant 1, \ldots, \mu_{d} \geqslant 1} \sum_{\mu_{1} \lambda_{1}+\cdots+\mu_{d} \lambda_{d} \leqslant \kappa+1} \\
& \sum_{l_{1: 1}=1}^{m} \ldots \sum_{l_{1: \mu_{1}}=1}^{m} \ldots \ldots \sum_{l_{d: 1}=1}^{m} \ldots \sum_{l_{d: \mu_{d}}=1}^{m}[?] \\
& \prod_{1 \leqslant \nu_{1} \leqslant \mu_{1}} y_{\lambda_{1}}^{l_{1: \nu_{1}}} \ldots \ldots \prod_{1 \leqslant \nu_{d} \leqslant \mu_{d}} y_{\lambda_{d}}^{l_{d: \nu_{d}}},
\end{aligned}
$$

where the term in brackets [?] is still unknown. To determine it, let us examine a few instances.

According to (4.8) (fourth line), the term $\left[6 \mathcal{Y}_{x^{2} y}-4 \mathcal{X}_{x^{3}}\right] y_{2}$ of $\mathbf{Y}_{4}$ developes as $\sum_{l_{1}=1}^{m}\left[6 \mathcal{Y}_{x^{2} y^{l_{1}}}^{j}-\delta_{l_{1}}^{j} 4 \mathcal{X}_{x^{3}}\right] y_{2}^{l_{1}}$ in $\mathbf{Y}_{4}^{j}$. Here, $6 \mathcal{Y}_{x^{2} y}$ just becomes $6 \mathcal{Y}_{x^{2} y^{l_{1}}}^{j}$. Thus, we

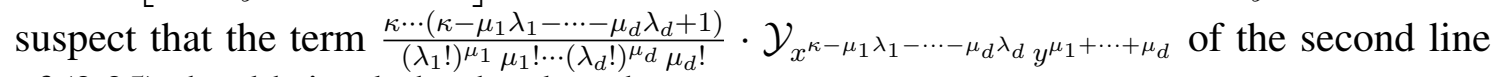
of (2.25) should simply be developed as

$$
\begin{aligned}
& \frac{\kappa(\kappa-1) \cdots\left(\kappa-\mu_{1} \lambda_{1}-\cdots-\mu_{d} \lambda_{d}+1\right)}{\left(\lambda_{1} !\right)^{\mu_{1}} \mu_{1} ! \cdots\left(\lambda_{d} !\right)^{\mu_{d}} \mu_{d} !} . \\
& \cdot \frac{\partial^{\kappa-\mu_{1} \lambda_{1}-\cdots-\mu_{d} \lambda_{d}+\mu_{1}+\cdots+\mu_{d}} \mathcal{Y}^{j}}{(\partial x)^{\kappa-\mu_{1} \lambda_{1}-\cdots-\mu_{d} \lambda_{d}} \partial y^{l_{1: 1}} \cdots \partial y^{l_{1: \mu_{1}}} \cdots \partial y^{l_{d: 1}} \cdots \partial y^{l_{d: \mu_{d}}}} .
\end{aligned}
$$

This rule is confirmed by inspecting all the other monomials of $\mathbf{Y}_{1}^{j}$, of $\mathbf{Y}_{2}^{j}$, of $\mathbf{Y}_{3}^{j}$ and of $\mathbf{Y}_{4}^{j}$.

It remains to determine how we must develope the term in $\mathcal{X}$ appearing in the last two lines of (2.25). To begin with, let us rewrite in advance this term in the slightly different shape, emphasizing a factorization:

$$
\frac{\kappa \cdots\left(\kappa-\mu_{1} \lambda_{1}-\cdots-\mu_{d} \lambda_{d}+2\right)}{\left(\lambda_{1} !\right)^{\mu_{1}} \mu_{1} ! \cdots\left(\lambda_{d} !\right)^{\mu_{d}} \mu_{d} !}\left[\left(\mu_{1} \lambda_{1}+\cdots+\mu_{d} \lambda_{d}\right) \mathcal{X}_{x^{\kappa-\mu_{1} \lambda_{1}-\cdots-\mu_{d} \lambda_{d}^{+1}} y^{\mu_{1}+\cdots+\mu_{d}-1}}\right] .
$$


Then we examine four instances extracted from the complete expression of $\mathbf{Y}_{4}^{j}$ :

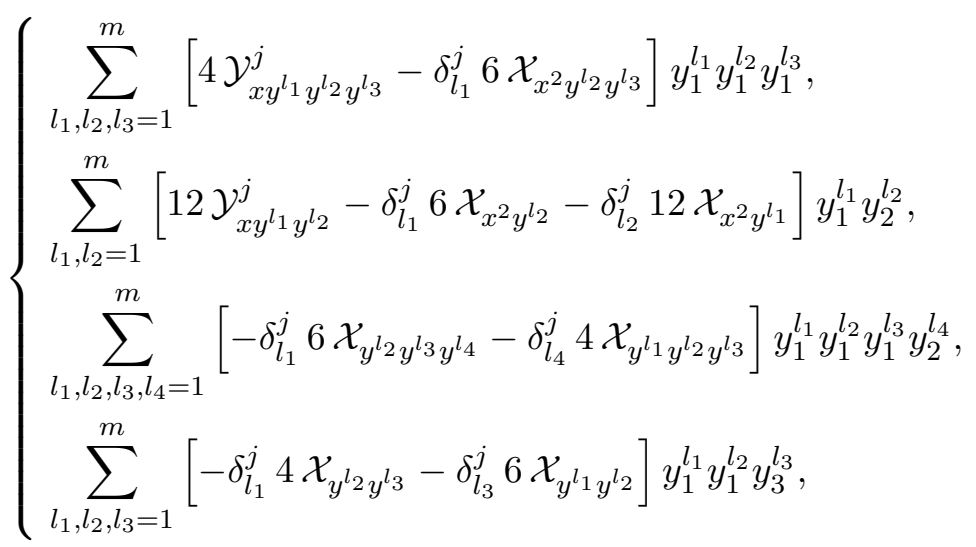

and we compare them to the corresponding terms of $\mathbf{Y}_{4}$ :

$$
\left\{\begin{array}{l}
{\left[4 \mathcal{Y}_{x y^{3}}-6 \mathcal{X}_{x^{2} y^{2}}\right]\left(y_{1}\right)^{3},} \\
{\left[12 \mathcal{Y}_{x y^{2}}-18 \mathcal{X}_{x^{2} y}\right] y_{1} y_{2},} \\
{\left[-10 \mathcal{X}_{y^{3}}\right]\left(y_{1}\right)^{3} y_{2},} \\
{\left[-10 \mathcal{X}_{y^{2}}\right]\left(y_{1}\right)^{2} y_{3}}
\end{array}\right.
$$

In the development from (4.16) to (4.15), we see that the four integers just before $\mathcal{X}$, namely $6=6,18=6+12,10=6+4$ and $10=4+6$, are split in a certain manner. Also, a single Kronecker symbol $\delta_{l_{\alpha}}^{j}$ is added as a factor. What are the rules?

In the second splitting $18=6+12$, we see that the relative weight of 6 and of 12 is the same as the relative weight of 1 and 2 in the splitting $3=1+2$ issued from the lower indices of the corresponding monomial $y_{1}^{l_{1}} y_{2}^{l_{2}}$. Similarly, in the third splitting $10=6+4$, the relative weight of 6 and of 4 is the same as the relative weight of $1+1+1$ and of 2 issued from the lower indices of the corresponding monomial $y_{1}^{l_{1}} y_{1}^{l_{2}} y_{1}^{l_{3}} y_{2}^{l_{4}}$. This rule may be confirmed by inspecting all the other monomials of $\mathbf{Y}_{2}, \mathbf{Y}_{2}^{j}$, of $\mathbf{Y}_{3}, \mathbf{Y}_{3}^{j}$ and of $\mathbf{Y}_{4}, \mathbf{Y}_{4}^{j}$. For a general $\kappa \geqslant 1$, the splitting of integers just amounts to decompose the sum appearing inside the brackets of (4.14) as $\mu_{1} \lambda_{1}, \mu_{2} \lambda_{2}, \ldots, \mu_{d} \lambda_{d}$. In fact, when we wrote (4.14), we emphasized in advance the decomposable factor $\left(\mu_{1} \lambda_{1}+\cdots+\mu_{d} \lambda_{d}\right)$.

Next, we have to determine what is the subscript $\alpha$ in the Kronecker symbol $\delta_{l_{\alpha}}^{j}$. We claim that in the four instances (4.15), the subscript $\alpha$ is intrinsically related to weight splitting. Indeed, recall that in the second line of (4.15), the number 6 of the splitting $18=6+12$ is related to the number 1 in the splitting $3=1+2$ of the lower indices of the monomial $y_{1}^{l_{1}} y_{2}^{l_{2}}$. It follows that the index $l_{\alpha}$ must be the index $l_{1}$ of the monomial $y_{1}^{l_{1}}$. Similarly, also in the second line of (4.15), the number 12 of the splitting $18=6+12$ being related to the number 2 in the splitting $3=1+2$ of the lower indices of the monomial $y_{1}^{l_{1}} y_{2}^{l_{2}}$, it follows that the index $l_{\alpha}$ attached to the second $\mathcal{X}$ term must be the index $l_{2}$ of the monomial $y_{2}^{l_{2}}$.

This rule is still ambiguous. Indeed, let us examine the third line of (4.15). We have the splitting $10=6+4$, homologous to the splitting of relative weights $5=(1+1+1)+2$ in the monomial $y_{1}^{l_{1}} y_{1}^{l_{2}} y_{1}^{l_{3}} y_{2}^{l_{4}}$. Of course, it is clear that we must choose the index $l_{4}$ for the Kronecker symbol associated to the second $\mathcal{X}$ term $-4 \mathcal{X}_{y^{3}}$, thus obtaining $-\delta_{l_{4}}^{j} 4 \mathcal{X}_{y^{l_{1}} y_{2} y_{2} l_{3}}$. However, since the monomial $y_{1}^{l_{1}} y_{1}^{l_{2}} y_{1}^{l_{3}}$ has three indices $l_{1}, l_{2}$ and $l_{3}$, there arises a question: what index $l_{\alpha}$ must we choose for the Kronecker symbol $\delta_{l_{\alpha}}^{j}$ attached to the first $\mathcal{X}$ term $6 \mathcal{X}_{y^{3}}$ : the index $l_{1}$, the index $l_{2}$ or the index $l_{3}$ ?

The answer is simple: any of the three indices $l_{1}, l_{2}$ or $l_{3}$ works. Indeed, since the monomial $y_{1}^{l_{1}} y_{1}^{l_{2}} y_{1}^{l_{3}}$ is symmetric with respect to all permutations of the set of three indices 
$\left\{l_{1}, l_{2}, l_{3}\right\}$, we have

(4.17)

$$
\begin{aligned}
\sum_{l_{1}, l_{2}, l_{3}, l_{4}=1}^{m}\left[-\delta_{l_{1}}^{j} 6 \mathcal{X}_{y^{l_{2}} y_{3} y^{l_{4}}}\right] y_{1}^{l_{1}} y_{1}^{l_{2}} y_{1}^{l_{3}} y_{2}^{l_{4}} & =\sum_{l_{1}, l_{2}, l_{3}, l_{4}=1}^{m}\left[-\delta_{l_{2}}^{j} 6 \mathcal{X}_{y^{l_{1}} y^{l_{3}} y^{l_{4}}}\right] y_{1}^{l_{1}} y_{1}^{l_{2}} y_{1}^{l_{3}} y_{2}^{l_{4}}= \\
& =\sum_{l_{1}, l_{2}, l_{3}, l_{4}=1}^{m}\left[-\delta_{l_{3}}^{j} 6 \mathcal{X}_{y^{l_{1}} y^{l_{2}} y^{l_{4}}}\right] y_{1}^{l_{1}} y_{1}^{l_{2}} y_{1}^{l_{3}} y_{2}^{l_{4}} .
\end{aligned}
$$

In fact, we have systematically used such symmetries during the intermediate computations (not exposed here) which we achieved manually to obtain the final expressions of $\mathbf{Y}_{1}^{j}$, of $\mathbf{Y}_{2}^{j}$, of $\mathbf{Y}_{3}^{j}$ and of $\mathbf{Y}_{4}^{j}$. To fix ideas, we have always choosen the first index. Here, the first index is $l_{1}$; in the first sum of line 9 of (4.8), the first index $l_{\alpha}$ for the second weight 12 is $l_{2}$.

This rule may be confirmed by inspecting all the monomials of $\mathbf{Y}_{2}^{j}$, of $\mathbf{Y}_{3}^{j}$, of $\mathbf{Y}_{4}^{j}$ (and also of $\mathbf{Y}_{5}^{j}$, which we have computed in a manuscript, but not copied in this Latex file).

From these considerations, we deduce that for the general formula, the weight decomposition is simply $\mu_{1} \lambda_{1}, \ldots, \mu_{d} \lambda_{d}$ and that the Kronecker symbol $\delta_{\alpha}^{j}$ is intrinsically associated to the weights. In conclusion, building on inductive reasonings, we have formulated the following statement.

Theorem 4.18. For one independent variable $x$, for several dependent variables $\left(y^{1}, \ldots, y^{m}\right)$ and for $\kappa \geqslant 1$, the general expression of the coefficient $\mathbf{Y}_{\kappa}^{j}$ of the prolongation (4.3) of a vector field is:

(4.19)

$$
\begin{aligned}
& \mathbf{Y}_{\kappa}^{j}=\mathcal{Y}_{x^{\kappa}}^{j}+\sum_{d=1}^{\kappa+1} \sum_{1 \leqslant \lambda_{1}<\cdots<\lambda_{d} \leqslant \kappa} \sum_{\mu_{1} \geqslant 1, \ldots, \mu_{d} \geqslant 1} \sum_{\mu_{1} \lambda_{1}+\cdots+\mu_{d} \lambda_{d} \leqslant \kappa+1} \\
& \sum_{l_{1: 1}=1}^{m} \cdots \sum_{l_{1: \mu_{1}}=1}^{m} \cdots \cdots \sum_{l_{d: 1}=1}^{m} \cdots \sum_{l_{d: \mu_{d}}=1}^{m} \frac{\kappa(\kappa-1) \cdots\left(\kappa-\mu_{1} \lambda_{1}+\cdots+\mu_{d} \lambda_{d}+2\right)}{\left(\lambda_{1} !\right)^{\mu_{1}} \mu_{1} ! \cdots\left(\lambda_{d} !\right)^{\mu_{d}} \mu_{d} !} \\
& {\left[\begin{array}{c}
\left(\kappa-\mu_{1} \lambda_{1}-\cdots-\mu_{d} \lambda_{d}+1\right) \frac{\partial^{\kappa-\mu_{1} \lambda_{1}-\cdots-\mu_{d} \lambda_{d}+\mu_{1}+\cdots+\mu_{d}} \mathcal{Y}^{j}}{(\partial x)^{\kappa-\mu_{1} \lambda_{1}-\cdots-\mu_{d} \lambda_{d}} \partial y^{l_{1: 1}} \cdots \partial y^{l_{1: \mu_{1}}} \cdots \partial y^{l_{d: 1}} \cdots \partial y^{l_{d: \mu_{d}}}}- \\
-\delta_{l_{1: 1}}^{j} \mu_{1} \lambda_{1} \frac{\partial^{\kappa-\mu_{1} \lambda_{1}-\cdots-\mu_{d} \lambda_{d}+\mu_{1}+\cdots+\mu_{d}} \mathcal{X}}{(\partial x)^{\kappa-\mu_{1} \lambda_{1}-\cdots-\mu_{d} \lambda_{d}+1} \widehat{\partial y^{l_{1: 1}}} \cdots \partial y^{l_{1: \mu_{1}}} \cdots \partial y^{l_{d: 1}} \cdots \partial y^{l_{d: \mu_{d}}}}- \\
-\cdots- \\
-\delta_{l_{d: 1}}^{j} \mu_{d} \lambda_{d} \frac{\partial^{\kappa-\mu_{1} \lambda_{1}-\cdots-\mu_{d} \lambda_{d}+\mu_{1}+\cdots+\mu_{d}} \mathcal{X}}{(\partial x)^{\kappa-\mu_{1} \lambda_{1}-\cdots-\mu_{d} \lambda_{d}+1} \partial y^{l_{1: 1}} \cdots \partial y^{l_{1: \mu_{1}}} \cdots \widehat{\partial y^{l_{d: 1}}} \cdots \partial y^{l_{d: \mu_{d}}}}
\end{array}\right]} \\
& \cdot \prod_{1 \leqslant \nu_{1} \leqslant \mu_{1}} y_{\lambda_{1}}^{l_{1: \nu_{1}}} \ldots \ldots \prod_{1 \leqslant \nu_{d} \leqslant \mu_{d}} y_{\lambda_{d}}^{l_{d: \nu_{d}}} \text {. }
\end{aligned}
$$

Here, the notation $\widehat{\partial y^{l}}$ means that the partial derivative is dropped.

Since the fundamental monomials appearing in the last line of (4.19) just above are not independent of each other, this formula has still to be modified a little bit. We refer to Section 6 for details.

4.20. Deduction of a multivariate Faà di Bruno formula. Let $m \in \mathbb{N}$ with $m \geqslant 1$, let $y=\left(y^{1}, \ldots, y^{m}\right) \in \mathbb{K}^{m}$, let $f=f\left(y^{1}, \ldots, y^{m}\right)$ be a $\mathcal{C}^{\infty}$-smooth function from $\mathbb{K}^{m}$ to $\mathbb{K}$, let $x \in \mathbb{K}$ and let $g^{1}=g^{1}(x), \ldots, g^{m}=g^{m}(x)$ be $\mathcal{C}^{\infty}$ functions from $\mathbb{K}$ to $\mathbb{K}$. The goal is to obtain an explicit formula for the derivatives, with respect to $x$, of the composition 
$h:=f \circ g$, namely $h(x):=f\left(g^{1}(x), \ldots, g^{m}(x)\right)$. For $\lambda \in \mathbb{N}$ with $\lambda \geqslant 1$, and for $j=1, \ldots, m$, we shall abbreviate the derivative $\frac{d^{\lambda} g^{j}}{d x^{\lambda}}$ by $g_{\lambda}^{j}$ and similarly for $h_{\lambda}$. The partial derivatives $\frac{\partial^{\lambda} f}{\partial y^{l_{1} \ldots \partial y^{l_{\lambda}}}}$ will be abbreviated by $f_{l_{1}, \ldots, l_{\lambda}}$.

Appying the chain rule, we may compute:

$$
\begin{aligned}
h_{1}= & \sum_{l_{1}=1}^{m} f_{l_{1}} g_{1}^{l_{1}}, \\
h_{2}= & \sum_{l_{1}, l_{2}=1}^{m} f_{l_{1}, l_{2}} g_{1}^{l_{1}} g_{1}^{l_{2}}+\sum_{l_{1}=1}^{m} f_{l_{1}} g_{2}^{l_{1}}, \\
h_{3}= & \sum_{l_{1}, l_{2}, l_{3}=1}^{m} f_{l_{1}, l_{2}, l_{3}} g_{1}^{l_{1}} g_{1}^{l_{2}} g_{1}^{l_{3}}+3 \sum_{l_{1}, l_{2}=1}^{m} f_{l_{1}, l_{2}} g_{1}^{l_{1}} g_{2}^{l_{2}}+\sum_{l_{1}=1}^{m} f_{l_{1}} g_{3}^{l_{1}}, \\
h_{4}= & \sum_{l_{1}, l_{2}, l_{3}, l_{4}=1}^{m} f_{l_{1}, l_{2}, l_{3}, l_{4}} g_{1}^{l_{1}} g_{1}^{l_{2}} g_{1}^{l_{3}} g_{1}^{l_{4}}+6 \sum_{l_{1}, l_{2}, l_{3}=1}^{m} f_{l_{1}, l_{2}, l_{3}} g_{1}^{l_{1}} g_{1}^{l_{2}} g_{2}^{l_{3}}+ \\
& +3 \sum_{l_{1}, l_{2}=1}^{m} f_{l_{1}, l_{2}} g_{2}^{l_{1}} g_{2}^{l_{2}}+4 \sum_{l_{1}, l_{2}=1}^{m} f_{l_{1}, l_{2}} g_{1}^{l_{1}} g_{3}^{l_{2}}+\sum_{l_{1}=1}^{m} f_{l_{1}} g_{4}^{l_{1}}, \\
& \sum_{l_{1}, l_{2}, l_{3}, l_{4}, l_{5}=1}^{m} f_{l_{1}, l_{2}, l_{3}, l_{4}, l_{5}} g_{1}^{l_{1}} g_{1}^{l_{2}} g_{1}^{l_{3}} g_{1}^{l_{4}} g_{1}^{l_{5}}+10 \sum_{l_{1}, l_{2}, l_{3}, l_{4}=1}^{m} f_{l_{1}, l_{2}, l_{3}, l_{4}} g_{1}^{l_{1}} g_{1}^{l_{2}} g_{1}^{l_{3}} g_{2}^{l_{4}}+ \\
& +15 \sum_{l_{1}, l_{2}, l_{3}=1}^{m} f_{l_{1}, l_{2}, l_{3}} g_{1}^{l_{1}} g_{2}^{l_{2}} g_{2}^{l_{3}}+10 \sum_{l_{1}, l_{2}, l_{3}=1}^{m} f_{l_{1}, l_{2}, l_{3}} g_{1}^{l_{1}} g_{1}^{l_{2}} g_{3}^{l_{3}}+ \\
& +10 \sum_{l_{1}, l_{2}=1}^{m} f_{l_{1}, l_{2}} g_{2}^{l_{1}} g_{3}^{l_{2}}+5 \sum_{l_{1}, l_{2}=1}^{m} f_{l_{1}, l_{2}} g_{1}^{l_{1}} g_{4}^{l_{2}}+\sum_{l_{1}=1}^{m} f_{l_{1}} g_{5}^{l_{1}} .
\end{aligned}
$$

Introducing the derivations

(4.22)

$$
\begin{aligned}
& F^{2}:=\sum_{l_{1}=1}^{m} g_{2}^{l_{1}} \frac{\partial}{\partial g_{1}^{l_{1}}}+\sum_{l_{1}=1}^{m} g_{1}^{l_{1}}\left(\sum_{l_{2}=1}^{m} f_{l_{1}, l_{2}} \frac{\partial}{\partial f_{l_{2}}}\right) \\
& F^{3}:=\sum_{l_{1}=1}^{m} g_{2}^{l_{1}} \frac{\partial}{\partial g_{1}^{l_{1}}}+\sum_{l_{1}=1}^{m} g_{3}^{l_{1}} \frac{\partial}{\partial g_{2}^{l_{1}}}+\sum_{l_{1}=1}^{m} g_{1}^{l_{1}}\left(\sum_{l_{2}=1}^{m} f_{l_{1}, l_{2}} \frac{\partial}{\partial f_{l_{2}}}+\sum_{l_{2}, l_{3}=1}^{m} f_{l_{1}, l_{2}, l_{3}} \frac{\partial}{\partial f_{l_{2}, l_{3}}}\right), \\
& F^{\lambda}:=\sum_{l_{1}=1}^{m} g_{2}^{l_{1}} \frac{\partial}{\partial g_{1}^{l_{1}}}+\sum_{l_{1}=1}^{m} g_{3}^{l_{1}} \frac{\partial}{\partial g_{2}^{l_{1}}}+\cdots+\sum_{l_{1}=1}^{m} g_{\lambda}^{l_{1}} \frac{\partial}{\partial g_{\lambda-1}^{l_{1}}}+ \\
& +\sum_{l_{1}=1}^{m} g_{1}^{l_{1}}\left(\sum_{l_{2}=1}^{m} f_{l_{1}, l_{2}} \frac{\partial}{\partial f_{l_{2}}}+\sum_{l_{2}, l_{3}=1}^{m} f_{l_{1}, l_{2}, l_{3}} \frac{\partial}{\partial f_{l_{2}, l_{3}}}+\cdots+\sum_{l_{2}, \ldots, l_{\lambda}=1}^{m} f_{l_{1}, l_{2}, \ldots, l_{\lambda}} \frac{\partial}{\partial f_{l_{2}, \ldots, l_{\lambda}}}\right),
\end{aligned}
$$

we observe that the following induction relations hold:

$$
\begin{aligned}
h_{2} & =F^{2}\left(h_{1}\right), \\
h_{3} & =F^{3}\left(h_{2}\right), \\
\ldots & \cdots \cdots . . . . \\
h_{\lambda}= & F^{\lambda}\left(h_{\lambda-1}\right) .
\end{aligned}
$$


To obtain the explicit version of the Faà di Bruno in the case of one variable $x$ and several variables $\left(y^{1}, \ldots, y^{m}\right)$, it suffices to extract from the expression of $\mathbf{Y}_{\kappa}^{j}$ provided by Theorem 4.18 only the terms corresponding to $\mu_{1} \lambda_{1}+\cdots+\mu_{d} \lambda_{d}=\kappa$, dropping all the $\mathcal{X}$ terms. After some simplifications and after a translation by means of an elementary dictionary, we may formulate a statement.

Theorem 4.24. For every integer $\kappa \geqslant 1$, the $\kappa$-th partial derivative of the composite function $h=h(x)=f\left(g^{1}(x), \ldots, g^{m}(x)\right)$ with respect to $x$ may be expressed as an explicit polynomial depending on the partial derivatives of $f$, on the derivatives of $g$ and having integer coefficients:

$$
\begin{gathered}
\frac{d^{\kappa} h}{d x^{\kappa}}=\sum_{d=1}^{\kappa} \sum_{\substack{1 \leqslant \lambda_{1}<\cdots<\lambda_{d} \leqslant \kappa \\
\mu_{1} \geqslant 1, \ldots, \mu_{d} \geqslant 1}} \sum_{\sum_{l_{1}, 1, \ldots, l_{1: \mu_{1}}=1}^{m} \cdots \sum_{l_{d: 1}, \ldots, l_{d: \mu_{d}}=1}^{m}} \frac{\kappa !}{\left(\lambda_{1} !\right)^{\mu_{1}} \mu_{1} ! \cdots\left(\lambda_{d} !\right)^{\mu_{d}} \mu_{d} !} \\
\frac{\partial^{\mu_{1}+\cdots+\mu_{d}} f}{\partial y^{l_{1: 1}} \cdots \partial y^{l_{1: \mu_{1}} \cdots \partial y^{l_{d: 1}} \cdots \partial y^{l_{d: \mu_{d}}}}} \prod_{1 \leqslant \nu_{1} \leqslant \mu_{1}} \frac{d^{\lambda_{1}} g^{l_{1: \nu_{1}}}}{d x^{\lambda_{1}}} \cdots \prod_{1 \leqslant \nu_{d} \leqslant \mu_{d}} \frac{d^{\lambda_{d}} g^{l_{d: \nu_{d}}}}{d x^{\lambda_{d}}} .
\end{gathered}
$$

We refer to Section 6 for the final writing of the above formula (4.25).

\section{§5. SEVERAL INDEPENDENT VARIABLES AND SEVERAL DEPENDENT VARIABLES}

5.1. Expression of $\mathbf{Y}_{i_{1}}^{j}$, of $\mathbf{Y}_{i_{1}, i_{2}}^{j}$ and of $\mathbf{Y}_{i_{1}, i_{2}, i_{3}}^{j}$. Applying the induction (1.31) and working out the obtained formulas until they take a perfect shape, we obtain firstly:

(5.2) $\mathbf{Y}_{i_{1}}^{j}=\mathcal{Y}_{x^{i_{1}}}^{j}+\sum_{l_{1}=1}^{m} \sum_{k_{1}=1}^{n}\left[\delta_{i_{1}}^{k_{1}} \mathcal{Y}_{y^{l_{1}}}^{j}-\delta_{l_{1}}^{j} \mathcal{X}_{x^{i_{1}}}^{k_{1}}\right] y_{k_{1}}^{l_{1}}+\sum_{l_{1}, l_{2}=1}^{m} \sum_{k_{1}, k_{2}=1}^{n}\left[-\delta_{l_{2}}^{j} \delta_{i_{1}}^{k_{1}} \mathcal{X}_{y^{l_{1}}}^{k_{2}}\right] y_{k_{1}}^{l_{1}} y_{k_{2}}^{l_{2}}$.

Secondly:

$$
\begin{aligned}
\mathbf{Y}_{i_{1}, i_{2}}^{j}= & \mathcal{Y}_{x^{i_{1}} x^{i_{2}}}^{j}+\sum_{l_{1}=1}^{m} \sum_{k_{1}=1}^{n}\left[\delta_{i_{1}}^{k_{1}} \mathcal{Y}_{x^{i_{2}} y^{l_{1}}}^{j}+\delta_{i_{2}}^{k_{1}} \mathcal{Y}_{x^{i_{1}} y^{l_{1}}}^{j}-\delta_{l_{1}}^{j} \mathcal{X}_{x^{i_{1}} x^{i_{2}}}^{k_{1}}\right] y_{k_{1}}^{l_{1}}+ \\
& +\sum_{l_{1}, l_{2}=1}^{m} \sum_{k_{1}, k_{2}=1}^{n}\left[\delta_{i_{1}, i_{2}}^{k_{1}, k_{2}} \mathcal{Y}_{y^{l_{1}} y^{l_{2}}}^{j}-\delta_{l_{2}}^{j} \delta_{i_{1}}^{k_{1}} \mathcal{X}_{x^{i_{2}} y^{l_{1}}}^{k_{2}}-\delta_{l_{2}}^{j} \delta_{i_{2}}^{k_{1}} \mathcal{X}_{x^{i_{1}} y^{l_{1}}}^{k_{2}}\right] y_{k_{1}}^{l_{1}} y_{k_{2}}^{l_{2}}+ \\
& +\sum_{l_{1}, l_{2}, l_{3}=1}^{m} \sum_{k_{1}, k_{2}, k_{3}=1}^{n}\left[-\delta_{l_{3}}^{j} \delta_{i_{1}, i_{2}}^{k_{1}, k_{2}} \mathcal{X}_{y^{l_{1}} y^{l_{2}}}^{k_{3}}\right] y_{k_{1}}^{l_{1}} y_{k_{2}}^{l_{2}} y_{k_{3}}^{l_{3}}+ \\
& +\sum_{l_{1}=1}^{m} \sum_{k_{1}, k_{2}=1}^{n}\left[\delta_{i_{1}, i_{2}}^{k_{1}, k_{2}} \mathcal{Y}_{y^{l_{1}}}^{j}-\delta_{l_{1}}^{j} \delta_{i_{1}}^{k_{1}} \mathcal{X}_{x^{i_{2}}}^{k_{2}}-\delta_{l_{1}}^{j} \delta_{i_{2}}^{k_{1}} \mathcal{X}_{x^{i_{1}}}^{k_{2}}\right] y_{k_{1}, k_{2}}^{l_{1}}+ \\
& +\sum_{l_{1}, l_{2}=1}^{m} \sum_{k_{1}, k_{2}, k_{3}=1}^{n}\left[-\delta_{l_{1}}^{j} \delta_{i_{1}, i_{2}}^{k_{2}, k_{3}} \mathcal{X}_{y^{l_{2}}}^{k_{1}}-\delta_{l_{2}}^{j} \delta_{i_{1}, i_{2}}^{k_{3}, k_{1}} \mathcal{X}_{y^{l_{1}}}^{k_{2}}-\delta_{l_{2}}^{j} \delta_{i_{1}, i_{2}}^{k_{1}, k_{2}} \mathcal{X}_{y_{1}}^{k_{3}}\right] y_{k_{1}}^{k_{1}} y_{k_{2}}^{l_{2}} y_{k_{3}}^{l_{3}} .
\end{aligned}
$$


Thirdly:

$$
\begin{aligned}
& \mathbf{Y}_{i_{1}, i_{2}, i_{3}}^{j}=\mathcal{Y}_{x^{i_{1}} x^{i_{2}} x^{i_{3}}}^{j}+\sum_{l_{1}=1}^{m} \sum_{k_{1}=1}^{n}\left[\delta_{i_{1}}^{k_{1}} \mathcal{Y}_{x^{i_{2}} x^{i_{3}} y^{l_{1}}}^{j}+\delta_{i_{2}}^{k_{1}} \mathcal{Y}_{x^{i_{1}} x^{i_{3}} y^{l_{1}}}^{j}+\delta_{i_{3}}^{k_{1}} \mathcal{Y}_{x^{i_{1}} x^{i_{2}} y^{l_{1}}}^{j}-\delta_{l_{1}}^{j} \mathcal{X}_{x^{i_{1}} x^{i_{2}} x^{i_{3}}}^{k_{1}}\right] y_{k_{1}}^{l_{1}}+ \\
& +\sum_{l_{1}, l_{2}=1}^{m} \sum_{k_{1}, k_{2}=1}^{n}\left[\delta_{i_{1}, i_{2}}^{k_{1}, k_{2}} \mathcal{Y}_{x^{i} 3}^{j} y^{l_{1}} y^{l_{2}}+\delta_{i_{3}, i_{1}}^{k_{1}, k_{2}} \mathcal{Y}_{x^{i_{2}} y^{l_{1}} y^{l_{2}}}^{j}+\delta_{i_{2}, i_{3}}^{k_{1}, k_{2}} \mathcal{Y}_{x^{i_{1}} y^{l_{1}} y^{l_{2}}}^{j}-\right. \\
& \left.-\delta_{l_{2}}^{j} \delta_{i_{1}}^{k_{1}} \mathcal{X}_{x^{i_{2}} x^{i_{3}} y^{l_{1}}}^{k_{1}}-\delta_{l_{2}}^{j} \delta_{i_{2}}^{k_{1}} \mathcal{X}_{x^{i_{1}} x^{i_{3}} y^{l_{1}}}^{k_{2}}-\delta_{l_{2}}^{j} \delta_{i_{3}}^{k_{1}} \mathcal{X}_{x^{i_{1}} x^{i_{2}} y^{l_{1}}}^{k_{2}}\right] y_{k_{1}}^{l_{1}} y_{k_{2}}^{l_{2}}+ \\
& +\sum_{l_{1}, l_{2}, l_{3}=1}^{m} \sum_{k_{1}, k_{2}, k_{3}=1}^{n}\left[\delta_{i_{1}, i_{2}, i_{3}}^{k_{1}, k_{2}, k_{3}} \mathcal{Y}_{y^{l_{1}} y^{l_{2}} y^{l_{3}}}^{j}-\delta_{l_{3}}^{j} \delta_{i_{1}, i_{2}}^{k_{1}, k_{2}} \mathcal{X}_{x^{i_{3}} y^{l_{1}} y^{l_{2}}}^{k_{3}}-\right. \\
& \left.-\delta_{l_{3}}^{j} \delta_{i_{1}, i_{3}}^{k_{1}, k_{2}} \mathcal{X}_{x^{i_{2}} y^{l_{1}} y^{l_{2}}}^{k_{3}}-\delta_{l_{3}}^{j} \delta_{i_{2}, i_{3}}^{k_{1}, k_{2}} \mathcal{X}_{x^{i_{1}} y^{l_{1}} y^{l_{2}}}^{k_{3}}\right] y_{k_{1}}^{l_{1}} y_{k_{2}}^{l_{2}} y_{k_{3}}^{l_{3}}+ \\
& +\sum_{l_{1}, l_{2}, l_{3}, l_{4}=1}^{m} \sum_{k_{1}, k_{2}, k_{3}, k_{4}=1}^{n}\left[-\delta_{l_{4}}^{j} \delta_{i_{1}, i_{2}, i_{3}}^{k_{1}, k_{2}, k_{3}} \mathcal{X}_{y^{l_{1}} y^{l_{2}} y^{l_{3}}}^{k_{4}}\right] y_{k_{1}}^{l_{1}} y_{k_{2}}^{l_{2}} y_{k_{3}}^{l_{3}} y_{k_{4}}^{l_{4}}+ \\
& +\sum_{l_{1}=1}^{m} \sum_{k_{1}, k_{2}=1}^{n}\left[\delta_{i_{1}, i_{2}}^{k_{1}, k_{2}} \mathcal{Y}_{x^{i_{3}} y^{l_{1}}}^{j}+\delta_{i_{3}, i_{1}}^{k_{1}, k_{2}} \mathcal{Y}_{x^{i_{2}} y^{l_{1}}}^{j}+\delta_{i_{2}, i_{3}}^{k_{1}, k_{2}} \mathcal{Y}_{x^{i_{1}} y^{l_{1}}}^{j}-\right. \\
& \left.-\delta_{l_{1}}^{j} \delta_{i_{1}}^{k_{1}} \mathcal{X}_{x^{i_{2}} x^{i_{3}}}^{k_{2}}-\delta_{l_{1}}^{j} \delta_{i_{2}}^{k_{1}} \mathcal{X}_{x^{i_{1}} x^{i_{3}}}^{k_{x_{2}}}-\delta_{l_{1}}^{j} \delta_{i_{3}}^{k_{1}} \mathcal{X}_{x^{i_{1}} x^{i_{2}}}^{k_{2}}\right] y_{k_{1}, k_{2}}^{l_{1}}+
\end{aligned}
$$

$$
\begin{aligned}
& +\sum_{l_{1}, l_{2}=1}^{m} \sum_{k_{1}, k_{2}, k_{3}=1}^{n}\left[\delta_{i_{1}, i_{2}, i_{3}}^{k_{1}, k_{2}, k_{3}} \mathcal{Y}_{y^{l_{1}} y^{l_{2}}}^{j}+\delta_{i_{1}, i_{2}, i_{3}}^{k_{3}, k_{1}, k_{2}} \mathcal{Y}_{y^{l_{1}} y^{l_{2}}}^{j}+\delta_{i_{1}, i_{2}, i_{3}}^{k_{2}, k_{3}, k_{1}} \mathcal{Y}_{y^{l_{1}} y^{l_{2}}}^{j}-\right. \\
& -\delta_{l_{1}}^{j} \delta_{i_{1}, i_{2}}^{k_{2}, k_{3}} \mathcal{X}_{x^{i_{3}} y^{l_{2}}}^{k_{1}}-\delta_{l_{1}}^{j} \delta_{i_{1}, i_{3}}^{k_{2}, k_{3}} \mathcal{X}_{x^{i_{2}} y^{l_{2}}}^{k_{1}}-\delta_{l_{1}}^{j} \delta_{i_{2}, i_{3}}^{k_{2}, k_{3}} \mathcal{X}_{x^{i_{1}} y^{l_{2}}}^{k_{1}}- \\
& -\delta_{l_{2}}^{j} \delta_{i_{1}, i_{2}}^{k_{3}, k_{1}} \mathcal{X}_{x^{i_{3}} y^{l_{1}}}^{k_{2}}-\delta_{l_{2}}^{j} \delta_{i_{1}, i_{3}}^{k_{3}, k_{1}} \mathcal{X}_{x^{i_{2}} y^{l_{1}}}^{k_{2}}-\delta_{l_{2}}^{j} \delta_{i_{2}, i_{3}}^{k_{3}, k_{1}} \mathcal{X}_{x^{i_{1}} y^{l_{1}}}^{k_{2}}- \\
& \left.-\delta_{l_{2}}^{j} \delta_{i_{1}, i_{2}}^{k_{1}, k_{2}} \mathcal{X}_{x^{i} y^{l_{1}}}^{k_{3}}-\delta_{l_{2}}^{j} \delta_{i_{1}, i_{3}}^{k_{1}, k_{2}} \mathcal{X}_{x^{i_{2}} y^{l_{1}}}^{k_{l_{3}}}-\delta_{l_{2}}^{j} \delta_{i_{2}, i_{3}}^{k_{1}, k_{2}} \mathcal{X}_{x^{i_{1}} y^{l_{1}}}^{k_{3}}\right] y_{k_{1}}^{l_{1}} y_{k_{2}, k_{3}}^{l_{2}}+ \\
& +\sum_{l_{1}, l_{2}, l_{3}=1}^{m} \sum_{k_{1}, k_{2}, k_{3}, k_{4}=1}^{n}\left[-\delta_{l_{3}}^{j} \delta_{i_{1}, i_{2}, i_{3}}^{k_{1}, k_{2}, k_{3}} \mathcal{X}_{y^{l_{1}} y^{l_{2}}}^{k_{4}}-\delta_{l_{3}}^{j} \delta_{i_{1}, i_{2}, i_{3}}^{k_{2}, k_{3}, k_{1}} \mathcal{X}_{y^{l_{1}} y^{l_{2}}}^{k_{4}}-\delta_{l_{3}}^{j} \delta_{i_{1}, i_{2}, i_{3}}^{k_{3}, k_{2}, k_{1}} \mathcal{X}_{y^{l_{1}} y^{l_{2}}}^{k_{4}}-\right. \\
& -\delta_{l_{2}}^{j} \delta_{i_{1}, i_{2}, i_{3}}^{k_{3}, k_{4}, k_{1}} \mathcal{X}_{y^{l_{1}} y^{l_{3}}}^{k_{2}}-\delta_{l_{2}}^{j} \delta_{i_{1}, i_{2}, i_{3}}^{k_{3}, k_{1}, k_{4}} \mathcal{X}_{y^{l_{1}} y^{l_{3}}}^{k_{2}}- \\
& \left.-\delta_{l_{2}}^{j} \delta_{i_{1}, i_{2}, i_{3}}^{k_{1}, k_{3}, k_{4}} \mathcal{X}_{y^{l_{1}} y^{l_{3}}}^{k_{2}}\right] y_{k_{1}}^{l_{1}} y_{k_{2}}^{l_{2}} y_{k_{3}, k_{4}}^{l_{3}}+ \\
& +\sum_{l_{1}, l_{2}=1}^{m} \sum_{k_{1}, k_{2}, k_{3}, k_{4}=1}^{n}\left[-\delta_{l_{2}}^{j} \delta_{i_{1}, i_{2}, i_{3}}^{k_{1}, k_{2}, k_{3}} \mathcal{X}_{y^{l_{1}}}^{k_{3}}-\delta_{l_{2}}^{j} \delta_{i_{1}, i_{2}, i_{3}}^{k_{2}, k_{4}, k_{1}} \mathcal{X}_{y^{l_{1}}}^{k_{3}}-\delta_{l_{2}}^{j} \delta_{i_{1}, i_{2}, i_{3}}^{k_{4}, k_{1}, k_{2}} \mathcal{X}_{y^{l_{1}}}^{k_{3}}\right] y_{k_{1}, k_{2}}^{l_{1}} y_{k_{3}, k_{4}}^{l_{2}}+ \\
& +\sum_{l_{1}=1}^{m} \sum_{k_{1}, k_{2}, k_{3}=1}^{n}\left[\delta_{i_{1}, i_{2}, 1_{3}}^{k_{1}, k_{2}, k_{3}} \mathcal{Y}_{y^{l_{1}}}^{j}-\delta_{l_{1}}^{j} \delta_{i_{1}, i_{2}}^{k_{1}, k_{2}} \mathcal{X}_{x^{i_{3}}}^{k_{3}}-\delta_{l_{1}}^{j} \delta_{i_{1}, i_{3}}^{k_{1}, k_{2}} \mathcal{X}_{x^{i_{2}}}^{k_{3}}-\delta_{l_{1}}^{j} \delta_{i_{2}, i_{3}}^{k_{1}, k_{2}} \mathcal{X}_{x^{i_{1}}}^{k_{3}}\right] y_{k_{1}, k_{2}, k_{3}}^{l_{1}}+ \\
& +\sum_{l_{1}, l_{2}=1}^{m} \sum_{k_{1}, k_{2}, k_{3}, k_{4}=1}^{n}\left[-\delta_{l_{2}}^{j} \delta_{i_{1}, i_{2}, i_{3}}^{k_{1}, k_{2}, k_{3}} \mathcal{X}_{y^{l_{1}}}^{k_{4}}-\delta_{l_{2}}^{j} \delta_{i_{1}, i_{2}, i_{3}}^{k_{4}, k_{1}, k_{2}} \mathcal{X}_{y^{l_{1}}}^{k_{3}}-\delta_{l_{2}}^{j} \delta_{i_{1}, i_{2}, i_{3}}^{k_{3}, k_{4}, k_{1}} \mathcal{X}_{y^{l_{1}}}^{k_{2}}-\right. \\
& \left.-\delta_{l_{1}}^{j} \delta_{i_{1}, i_{2}, i_{3}}^{k_{2}, k_{3}, k_{4}} \mathcal{X}_{y^{l_{2}}}^{k_{1}}\right] y_{k_{1}}^{l_{1}} y_{k_{2}, k_{3}, k_{4}}^{l_{2}}
\end{aligned}
$$

5.5. Final synthesis. To obtain the general formula for $\mathbf{Y}_{i_{1}, \ldots, i_{\kappa}}^{j}$, we have to achieve the synthesis between the two formulas (3.74) and (4.19). We start with (3.74) and we modify it until we reach the final formula for $\mathbf{Y}_{i_{1}, \ldots, i_{\kappa}}^{j}$. 
We have to add the $\mu_{1}+\cdots+\mu_{d}$ sums $\sum_{l_{1: 1}=1}^{m} \cdots \sum_{l_{1: \mu_{1}}=1}^{m} \cdots \cdots \sum_{l_{d: 1}=1}^{m} \cdots \sum_{l_{d: \mu_{d}=1}}^{m}$, together with various indices $l_{\alpha}$. About these indices, the only point which is not obvious may be analyzed as follows.

A permutation $\sigma \in \mathfrak{F}_{\mu_{1} \lambda_{1}+\cdots+\mu_{d} \lambda_{d}}^{\left(\mu_{1}, \lambda_{1}\right), \ldots,\left(\mu_{d}, \lambda_{d}\right)}$ yields the list:

$$
\begin{aligned}
& \sigma(1: 1: 1), \ldots, \sigma\left(1: 1: \lambda_{1}\right), \ldots \sigma\left(1: \mu_{1}: 1\right), \ldots, \sigma\left(1: \mu_{1}: \lambda_{1}\right), \ldots \\
& \ldots, \sigma(d: 1: 1), \ldots, \sigma\left(1: 1: \lambda_{d}\right), \ldots \sigma\left(d: \mu_{d}: 1\right), \ldots, \sigma\left(d: \mu_{d}: \lambda_{d}\right),
\end{aligned}
$$

In the sixth line of (3.74), the last term $\sigma\left(d: \mu_{d}: \lambda_{d}\right)$ of the above list appears as the subscript of the upper index $k_{\sigma\left(d: \mu_{d}: \lambda_{d}\right)}$ of the term $\mathcal{X}^{k_{\sigma\left(d: \mu_{d}: \lambda_{d}\right)} \text {. According to the formal }}$ rules of Theorem 4.19, we have to multiply the partial derivative of $\mathcal{X}^{k_{\sigma\left(d: \mu_{d}: \lambda_{d}\right)}}$ by a certain Kronecker symbol $\delta_{l_{\alpha}}^{j}$. The question is: what is the subscript $\alpha$ and how to denote it?

As explained before the statement of Theorem 4.19, the subscript $\alpha$ is obtained as follows. The term $\sigma\left(d: \mu_{d}: \lambda_{d}\right)$ is of the form $\left(e: \nu_{d}: \gamma_{e}\right)$, for some $e$ with $1 \leqslant e \leqslant d$, for some $\nu_{e}$ with $1 \leqslant \nu_{e} \leqslant \mu_{e}$ and for some $\gamma_{e}$ with $1 \leqslant \gamma_{e} \leqslant \lambda_{e}$. The single pure jet variable

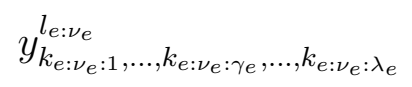

appears inside the total monomial

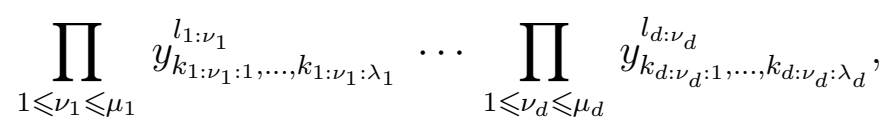

placed at the end of the formula for $\mathbf{Y}_{i_{1}, \ldots, i_{\kappa}}^{j}$ (see in advance formula (5.13) below; this total monomial generalizes to several dependent variables the total monomial appearing in the last line of (3.74)). According to the rule explained before the statement of Theorem 4.18, the index $l_{\alpha}$ must be equal to $l_{e: \nu_{e}}$, since $l_{e: \nu_{e}}$ is attached to the monomial (5.7). Coming back to the term $\sigma\left(d: \mu_{d}: \lambda_{d}\right)$, we shall denote this index by

$$
l_{e: \nu_{e}}=: l_{\pi\left(e: \nu_{e}: \gamma_{e}\right)}=: l_{\pi \sigma\left(d: \mu_{d}: \lambda_{d}\right)}
$$

where the symbol $\pi$ denotes the projection from the set

$$
\left\{1: 1: 1, \ldots, 1: \mu_{1}: \lambda_{1}, \ldots \ldots, d: 1: 1, \ldots, d: \mu_{d}: \lambda_{d}\right\}
$$

to the set

$$
\left\{1: 1, \ldots, 1: \mu_{1}, \ldots, d: 1, \ldots, d: \mu_{d}\right\}
$$

simply defined by $\pi\left(e: \nu_{e}: \gamma_{e}\right):=\left(e: \nu_{e}\right)$.

In conclusion, by means of this formalism, we may write down the complete generalization of Theorems 2.24, 3.73 and 4.18 to several independent variables and several dependent variables 
Theorem 5.12. For $j=1, \ldots, m$, for every $\kappa \geqslant 1$ and for every choice of $\kappa$ indices $i_{1}, \ldots, i_{\kappa}$ in the set $\{1,2, \ldots, n\}$, the general expression of $\mathbf{Y}_{i_{1}, \ldots, i_{\kappa}}^{j}$ is as follows: (5.13)

$$
\begin{aligned}
& \mathbf{Y}_{i_{1}, \ldots, i_{\kappa}}^{j}=\mathcal{Y}_{x^{i_{1} \ldots x^{i} \kappa}}^{j}+\sum_{d=1}^{\kappa+1} \sum_{1 \leqslant \lambda_{1}<\cdots<\lambda_{d} \leqslant \kappa} \sum_{\mu_{1} \geqslant 1, \ldots, \mu_{d} \geqslant 1} \sum_{\mu_{1} \lambda_{1}+\cdots+\mu_{d} \lambda_{d} \leqslant \kappa+1} \\
& \sum_{l_{1: 1}=1}^{m} \cdots \sum_{l_{1: \mu_{1}}=1}^{m} \cdots \cdots \sum_{l_{d: 1}=1}^{m} \ldots \sum_{l_{d: \mu_{d}}=1}^{m}
\end{aligned}
$$

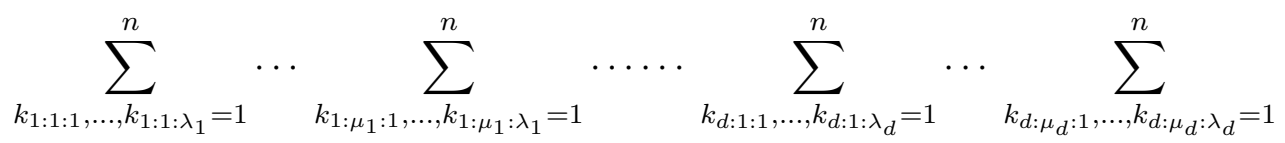

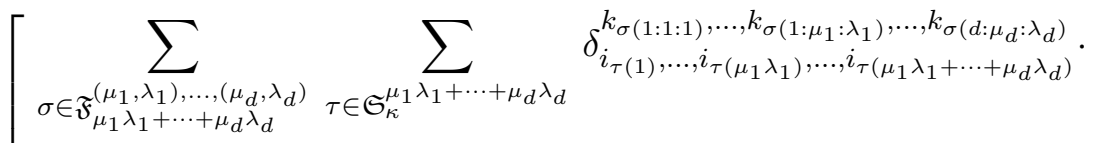

$$
\begin{aligned}
& \cdot \frac{\partial^{\kappa-\mu_{1} \lambda_{1}-\cdots-\mu_{d} \lambda_{d}+\mu_{1}+\cdots+\mu_{d}} \mathcal{Y}^{j}}{\partial x^{i_{\tau\left(\mu_{1} \lambda_{1}+\cdots+\mu_{d} \lambda_{d}+1\right)}} \cdots \partial x^{i_{\tau(\kappa)}} \partial y^{l_{1: 1}} \cdots \partial y^{l_{d: \mu_{d}}}}-
\end{aligned}
$$

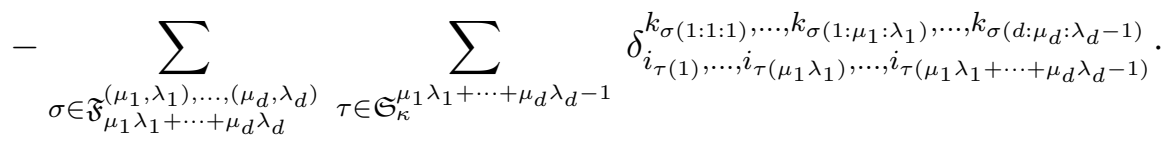

$$
\begin{aligned}
& \left.\cdot \delta_{l_{\pi \sigma\left(d: \mu_{d}: \lambda_{d}\right)}^{j}}^{j} \cdot \frac{\partial^{\kappa-\mu_{1} \lambda_{1}-\cdots-\mu_{d} \lambda_{d}+\mu_{1}+\cdots+\mu_{d}} \mathcal{X}^{k_{\sigma\left(d: \mu_{d}: \lambda_{d}\right)}}}{\partial x^{\left.i_{\tau\left(\mu_{1} \lambda_{1}+\cdots+\mu_{d} \lambda_{d}\right)}\right)} \cdots \partial x^{i_{\tau(\kappa)}} \partial y^{l_{1: 1}} \cdots \partial y^{\widehat{\left.l_{\pi\left(d: \mu_{d}: \lambda_{d}\right.}\right)}} \cdots \partial y^{l_{l_{d: \mu_{d}}}}}\right]
\end{aligned}
$$

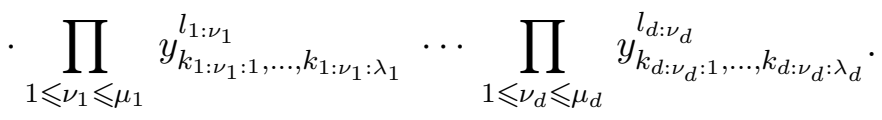

In this formula, the coset $\mathfrak{F}_{\mu_{1} \lambda_{1}+\cdots+\mu_{d} \lambda_{d}}^{\left(\mu_{1}, \lambda_{1}\right), \ldots,\left(\mu_{d}, \lambda_{d}\right)}$ was defined in equation (3.71); as in Theorem 3.73, we have made the identification:

$$
\{1, \ldots, \kappa\} \equiv\left\{1: 1: 1, \ldots, 1: \mu_{1}: \lambda_{1}, \ldots, \ldots, d: 1: 1, \ldots, d: \mu_{d}: \lambda_{d}\right\}
$$

Since the fundamental monomials appearing in the last line of (4.19) just above are not independent of each other, this formula has still to be modified a little bit. We refer to Section 6 for details.

5.15. Deduction of the most general multivariate Faà di Bruno formula. Let $n \in \mathbb{N}$ with $n \geqslant 1$, let $x=\left(x^{1}, \ldots, x^{n}\right) \in \mathbb{K}^{n}$, let $m \in \mathbb{N}$ with $m \geqslant 1$, let $g^{j}=g^{j}\left(x^{1}, \ldots, x^{n}\right)$, $j=1, \ldots, m$, be $\mathcal{C}^{\infty}$-smooth functions from $\mathbb{K}^{n}$ to $\mathbb{K}^{m}$, let $y=\left(y^{1}, \ldots, y^{m}\right) \in \mathbb{K}^{m}$ and let $f=f\left(y^{1}, \ldots, y^{m}\right)$ be a $\mathcal{C}^{\infty}$ function from $\mathbb{K}^{m}$ to $\mathbb{K}$. The goal is to obtain an explicit formula for the partial derivatives of the composition $h:=f \circ g$, namely

$$
h\left(x^{1}, \ldots, x^{n}\right):=f\left(g^{1}\left(x^{1}, \ldots, x^{n}\right), \ldots, g^{m}\left(x^{1}, \ldots, x^{n}\right)\right) .
$$

For $j=1, \ldots, m$, for $\lambda \in \mathbb{N}$ with $\lambda \geqslant 1$ and for arbitrary indices $i_{1}, \ldots, i_{\lambda}=1, \ldots, n$, we shall abbreviate the partial derivative $\frac{\partial^{\lambda} g^{j}}{\partial x^{i_{1} \ldots \partial x^{i} \lambda}}$ by $g_{i_{1}, \ldots, i_{\lambda}}^{j}$ and similarly for $h_{i_{1}, \ldots, i_{\lambda} \text {. For }}$ arbitrary indices $l_{1}, \ldots, l_{\lambda}=1, \ldots, m$, the partial derivative $\frac{\partial^{\lambda} f}{\partial y^{l_{1} \ldots \partial y^{l} \lambda}}$ will be abbreviated by $f_{l_{1}, \ldots, l_{\lambda}}$. 
Appying the chain rule, we may compute:

(5.17)

$$
\begin{aligned}
& h_{i_{1}}=\sum_{l_{1}=1}^{m} f_{l_{1}}\left[g_{i_{1}}^{l_{1}}\right] \\
& h_{i_{1}, i_{2}}=\sum_{l_{1}, l_{2}=1}^{m} f_{l_{1}, l_{2}}\left[g_{i_{1}}^{l_{1}} g_{i_{2}}^{l_{2}}\right]+\sum_{l_{1}=1}^{m} f_{l_{1}}\left[g_{i_{1}, i_{2}}^{l_{1}}\right] \text {, } \\
& h_{i_{1}, i_{2}, i_{3}}=\sum_{l_{1}, l_{2}, l_{3}=1}^{m} f_{l_{1}, l_{2}, l_{3}}\left[g_{i_{1}}^{l_{1}} g_{i_{2}}^{l_{2}} g_{i_{3}}^{l_{3}}\right]+\sum_{l_{1}, l_{2}=1}^{m} f_{l_{1}, l_{2}}\left[g_{i_{1}}^{l_{1}} g_{i_{2}, i_{3}}^{l_{2}}+g_{i_{2}}^{l_{1}} g_{i_{1}, i_{3}}^{l_{2}}+g_{i_{3}}^{l_{1}} g_{i_{1}, i_{2}}^{l_{2}}\right]+ \\
& +\sum_{l_{1}=1}^{m} f_{l_{1}}\left[g_{i_{1}, i_{2}, i_{3}}^{l_{1}}\right] \\
& h_{i_{1}, i_{2}, i_{3}, i_{4}}=\sum_{l_{1}, l_{2}, l_{3}, l_{4}=1}^{m} f_{l_{1}, l_{2}, l_{3}, l_{4}}\left[g_{i_{1}}^{l_{1}} g_{i_{2}}^{l_{2}} g_{i_{3}}^{l_{3}} g_{i_{4}}^{l_{4}}\right]+ \\
& \sum_{l_{1}, l_{2}, l_{3}=1}^{m} f_{l_{1}, l_{2}, l_{3}}\left[g_{i_{2}}^{l_{1}} g_{i_{3}}^{l_{2}} g_{i_{1}, i_{4}}^{l_{3}}+g_{i_{3}}^{l_{1}} g_{i_{1}}^{l_{2}} g_{i_{2}, i_{4}}^{l_{3}}+g_{i_{1}}^{l_{1}} g_{i_{2}}^{l_{2}} g_{i_{3}, i_{4}}^{l_{3}}+\right. \\
& \left.+g_{i_{1}}^{l_{1}} g_{i_{4}}^{l_{2}} g_{i_{2}, i_{3}}^{l_{3}}+g_{i_{2}}^{l_{1}} g_{i_{4}}^{l_{2}} g_{i_{3}, i_{1}}^{l_{3}}+g_{i_{3}}^{l_{1}} g_{i_{4}}^{l_{2}} g_{i_{1}, i_{2}}^{l_{3}}\right]+ \\
& +\sum_{l_{1}, l_{2}=1}^{m} f_{l_{1}, l_{2}}\left[g_{i_{1}, i_{2}}^{l_{1}} g_{i_{3}, i_{4}}^{l_{2}}+g_{i_{1}, i_{3}}^{l_{1}} g_{i_{2}, i_{4}}^{l_{2}}+g_{i_{1}, i_{4}}^{l_{1}} g_{i_{2}, i_{3}}^{l_{2}}\right]+ \\
& +\sum_{l_{1}, l_{2}=1}^{m} f_{l_{1}, l_{2}}\left[g_{i_{1}}^{l_{1}} g_{i_{2}, i_{3}, i_{4}}^{l_{2}}+g_{i_{2}}^{l_{1}} g_{i_{1}, i_{3}, i_{4}}^{l_{2}}+g_{i_{3}}^{l_{1}} g_{i_{1}, i_{2}, i_{4}}^{l_{2}}+g_{i_{4}}^{l_{1}} g_{i_{1}, i_{2}, i_{3}}^{l_{2}}\right]+ \\
& +\sum_{l_{1}=1}^{m} f_{l_{1}}\left[g_{i_{1}, i_{2}, i_{3}, i_{4}}^{l_{1}}\right] \text {. }
\end{aligned}
$$

Introducing the derivations

$$
\begin{aligned}
F_{i}^{2}:= & \sum_{k_{1}=1}^{n} \sum_{l_{1}=1}^{m} g_{k_{1}, i}^{l_{1}} \frac{\partial}{\partial g_{k_{1}}^{l_{1}}}+\sum_{l_{1}=1}^{m} g_{i}^{l_{1}}\left(\sum_{l_{2}=1}^{m} f_{l_{1}, l_{2}} \frac{\partial}{\partial f_{l_{2}}}\right), \\
F_{i}^{3}:= & \sum_{k_{1}=1}^{n} \sum_{l_{1}=1}^{m} g_{k_{1}, i}^{l_{1}} \frac{\partial}{\partial g_{k_{1}}^{l_{1}}}+\sum_{k_{1}, k_{2}=1}^{n} \sum_{l_{1}=1}^{m} g_{k_{1}, k_{2}, i}^{l_{1}} \frac{\partial}{\partial g_{k_{1}, k_{2}}^{l_{1}}}+ \\
& +\sum_{l_{1}=1}^{m} g_{i}^{l_{1}}\left(\sum_{l_{2}=1}^{m} f_{l_{1}, l_{2}} \frac{\partial}{\partial f_{l_{2}}}+\sum_{l_{2}, l_{3}=1}^{m} f_{l_{1}, l_{2}, l_{3}} \frac{\partial}{\partial f_{l_{2}, l_{3}}}\right),
\end{aligned}
$$




$$
\begin{aligned}
F_{i}^{\lambda}:= & \sum_{k_{1}=1}^{n} \sum_{l_{1}=1}^{m} g_{k_{1}, i}^{l_{1}} \frac{\partial}{\partial g_{k_{1}}^{l_{1}}}+\sum_{k_{1}, k_{2}=1}^{n} \sum_{l_{1}=1}^{m} g_{k_{1}, k_{2}, i}^{l_{1}} \frac{\partial}{\partial g_{k_{1}, k_{2}}^{l_{1}}}+\cdots+ \\
& +\sum_{k_{1}, k_{2}, \ldots, k_{\lambda-1}=1}^{n} \sum_{l_{1}=1}^{m} g_{k_{1}, k_{2}, \ldots, k_{\lambda-1}, i} \frac{\partial}{\partial g_{k_{1}, \ldots, k_{\lambda-1}}^{l_{1}}}+ \\
& +\sum_{l_{1}=1}^{m} g_{i}^{l_{1}}\left(\sum_{l_{2}=1}^{m} f_{l_{1}, l_{2}} \frac{\partial}{\partial f_{l_{2}}}+\sum_{l_{2}, l_{3}=1}^{m} f_{l_{1}, l_{2}, l_{3}} \frac{\partial}{\partial f_{l_{2}, l_{3}}}+\right. \\
& \left.+\cdots+\sum_{l_{2}, l_{3}, \ldots, l_{\lambda}} f_{l_{1}, l_{2}, l_{3}, \ldots, l_{\lambda}} \frac{\partial}{\partial f_{l_{2}, l_{3}, \ldots, l_{\lambda}}}\right),
\end{aligned}
$$

we observe that the following induction relations hold:

$$
\begin{aligned}
h_{i_{1}, i_{2}} & =F_{i_{2}}^{2}\left(h_{i_{1}}\right), \\
h_{i_{1}, i_{2}, i_{3}} & =F_{i_{3}}^{3}\left(h_{i_{1}, i_{2}}\right), \\
\ldots \ldots & \cdots \ldots \ldots \ldots \ldots \ldots . . . \\
h_{i_{1}, i_{2}, \ldots, i_{\lambda}} & =F_{i_{\lambda}}^{\lambda}\left(h_{i_{1}, i_{2}, \ldots, i_{\lambda-1}}\right) .
\end{aligned}
$$

To obtain the explicit version of the Faà di Bruno in the case of several variables $\left(x^{1}, \ldots, x^{n}\right)$ and several variables $\left(y^{1}, \ldots, y^{m}\right)$, it suffices to extract from the expression of $\mathbf{Y}_{i_{1}, \ldots, i_{\kappa}}^{j}$ provided by Theorem 5.12 only the terms corresponding to $\mu_{1} \lambda_{1}+\cdots+\mu_{d} \lambda_{d}=$ $\kappa$, dropping all the $\mathcal{X}$ terms. After some simplifications and after a translation by means of an elementary dictionary, we obtain the fourth and the most general multivariate Faà di Bruno formula.

Theorem 5.20. For every integer $\kappa \geqslant 1$ and for every choice of indices $i_{1}, \ldots, i_{\kappa}$ in the set $\{1,2, \ldots, n\}$, the $\kappa$-th partial derivative of the composite function

$$
h=h\left(x^{1}, \ldots, x^{n}\right)=f\left(g^{1}\left(x^{1}, \ldots, x^{n}\right), \ldots, g^{m}\left(x^{1}, \ldots, x^{n}\right)\right)
$$

with respect to the variables $x^{i_{1}}, \ldots, x^{i_{\kappa}}$ may be expressed as an explicit polynomial depending on the partial derivatives of $f$, on the partial derivatives of the $g^{j}$ and having integer coefficients:

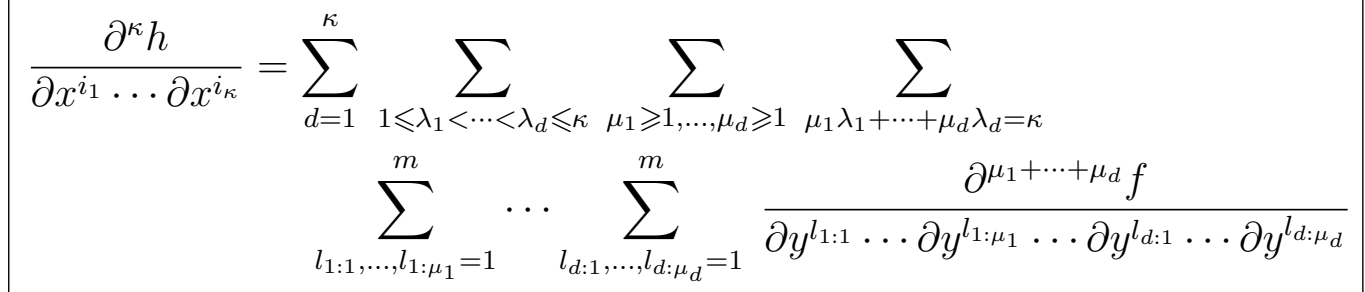

$$
\begin{aligned}
& {\left[\begin{array}{rl}
\sum_{\sigma \in \mathfrak{F}_{\kappa}^{\left(\mu_{1}, \lambda_{1}\right), \ldots,\left(\mu_{d}, \lambda_{d}\right)}} & \prod_{1 \leqslant \nu_{1} \leqslant \mu_{1}} \frac{\partial^{\lambda_{1}} g^{l_{1: \nu_{1}}}}{\partial x^{i_{\sigma\left(1: \nu_{1}: 1\right)}} \cdots \partial x^{i_{\sigma\left(1: \nu_{1}: \lambda_{1}\right)}}} \cdots \\
\cdots & \prod_{1 \leqslant \nu_{d} \leqslant \mu_{d}} \frac{\partial^{\lambda_{d}} g^{l_{d_{2} \nu_{d}}}}{\partial x^{i_{\sigma\left(d: \nu_{d}: 1\right)}} \cdots \partial x^{i_{\sigma\left(d: \nu_{d}: \lambda_{d}\right)}}}
\end{array}\right] .}
\end{aligned}
$$




\title{
III: Systems of second order
}

\author{
Table of contents
}

1. Explicit characterizations of flatness $\ldots \ldots \ldots \ldots \ldots \ldots \ldots \ldots \ldots \ldots \ldots \ldots \ldots \ldots .$,

2. Completely integrable systems of second order ordinary differential equations ... 100.

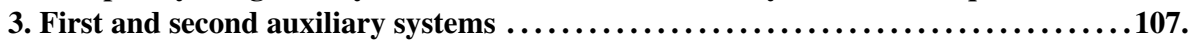

\section{§1. EXPLICIT CHARACTERIZATIONS OF FLATNESS}

In 1883, S. Lie obtained the following explicit characterization of the local equivalence of a second order ordinary differential equation $\left(\mathcal{E}_{1}\right): y_{x x}=F\left(x, y, y_{x}\right)$ to the Newtonian free particle equation with one degree of freedom $Y_{X X}=0$. All the functions are assumed to be analytic.

Theorem 1.1. ([Lie1883], pp. 362-365) Let $\mathbb{K}=\mathbb{R}$ of $\mathbb{C}$. Let $x \in \mathbb{K}$ and $y \in \mathbb{K}$. A local second order ordinary differential equation $y_{x x}=F\left(x, y, y_{x}\right)$ is equivalent under an invertible point transformation $(x, y) \mapsto(X(x, y), Y(x, y))$ to the free particle equation $Y_{X X}=0$ if and only if the following two conditions are satisfied:

(i) $F_{y_{x} y_{x} y_{x} y_{x}}=0$, or equivalently $F$ is a degree three polynomial in $y_{x}$, namely there exist four functions $G, H, L$ and $M$ of $(x, y)$ such that $F$ can be written as

$$
F\left(x, y, y_{x}\right)=G(x, y)+y_{x} \cdot H(x, y)+\left(y_{x}\right)^{2} \cdot L(x, y)+\left(y_{x}\right)^{3} \cdot M(x, y)
$$

(ii) the four functions $G, H, L$ and $M$ satisfy the following system of two second order quasi-linear partial differential equations:

$$
\left\{\begin{aligned}
0=-2 G_{y y} & +\frac{4}{3} H_{x y}-\frac{2}{3} L_{x x}+ \\
& +2(G L) y-2 G_{x} M-4 G M_{x}+\frac{2}{3} H L_{x}-\frac{4}{3} H H_{y}, \\
0=-\frac{2}{3} H_{y y} & +\frac{4}{3} L_{x y}-2 M_{x x}+ \\
& +2 G M_{y}+4 G_{y} M-2(H M)_{x}-\frac{2}{3} H_{y} L+\frac{4}{3} L L_{x} .
\end{aligned}\right.
$$

Open question 1.4. Deduce an explicit necessary and sufficient condition for the associated submanifold of solutions $y=\Pi(x, a, b)$ to be locally equivalent to $Y=B+X A$.

Assuming $F=F\left(x, y_{x}\right)$ to be independent of $y$, or equivalently assuming $\mathcal{M}_{\left(\mathcal{E}_{1}\right)}$ to be:

$$
y=b+\Pi(x, a)
$$


the author has checked that equivalence to $Y=B+X A$ holds if and only if two differential rational expressions annihilate:

$$
\begin{aligned}
0= & \frac{\Pi_{x^{2} a^{4}}}{\left(\Pi_{x a}\right)^{4}}-6 \frac{\Pi_{x^{2} a^{3}} \Pi_{x a^{2}}}{\left(\Pi_{x a}\right)^{5}}+15 \frac{\Pi_{x^{2} a^{2}}\left(\Pi_{x a^{2}}\right)^{2}}{\left(\Pi_{x a}\right)^{6}}-4 \frac{\Pi_{x^{2} a^{2}} \Pi_{x a^{3}}}{\left(\Pi_{x a}\right)^{5}} \\
& -\frac{\Pi_{x^{2} a} \Pi_{x a^{4}}}{\left(\Pi_{x a}\right)^{5}}+10 \frac{\Pi_{x a^{3}} \Pi_{x^{2} a} \Pi_{x a^{2}}}{\left(\Pi_{x a}\right)^{6}}-15 \frac{\Pi_{x^{2} a}\left(\Pi_{x a^{2}}\right)^{3}}{\left(\Pi_{x a}\right)^{7}} \text { and } \\
0= & \frac{\Pi_{x^{4} a^{2}}}{\left(\Pi_{x a}\right)^{2}}-6 \frac{\Pi_{x^{3} a^{2}} \Pi_{x^{2} a}}{\left(\Pi_{x a}\right)^{3}}-4 \frac{\Pi_{x^{3} a} \Pi_{x^{2} a^{2}}}{\left(\Pi_{x a}\right)^{3}}-\frac{\Pi_{x^{4} a} \Pi_{x a^{2}}}{\left(\Pi_{x a}\right)^{3}}+ \\
& +15 \frac{\Pi_{x^{2} a^{2}}\left(\Pi_{x^{2} a}\right)^{2}}{\left(\Pi_{x a}\right)^{4}}+10 \frac{\Pi_{x^{3} a} \Pi_{x^{2} a} \Pi_{x a^{2}}}{\left(\Pi_{x a}\right)^{4}}-15 \frac{\left(\Pi_{x^{2} a}\right)^{3} \Pi_{x a^{2}}}{\left(\Pi_{x a}\right)^{5}}
\end{aligned}
$$

As an application, this characterizes local sphericity of a rigid hypersurface $w=\bar{w}+$ $i \Theta(z, \bar{z})$ of $\mathbb{C}^{2}$. The answer for a general $y=\Pi(x, a, b)$, together with a proof, will appear elsewhere.

A modern restitution of Lie's original proof of Theorem 1.1 may be found in [Me2004]. In this reference, we generalize Theorem 1.1 to several dependent variables $y=$ $\left(y^{1}, y^{2}, \ldots, y^{m}\right)$. In the present Part III, we will instead pass to several independent variables $x=\left(x^{1}, x^{2}, \ldots, x^{n}\right)$.

Theorem 1.7. Let $\mathbb{K}=\mathbb{R}$ or $\mathbb{C}$, let $n \in \mathbb{N}$, suppose $n \geqslant 2$ and consider a system of completely integrable partial differential equations in $n$ independent variables $x=\left(x^{1}, \ldots, x^{n}\right) \in \mathbb{K}^{n}$ and in one dependent variable $y \in \mathbb{K}$ of the form:

$$
y_{x^{j_{1}} x^{j_{2}}}(x)=F^{j_{1}, j_{2}}\left(x, y(x), y_{x^{1}}(x), \ldots, y_{x^{n}}(x)\right), \quad 1 \leqslant j_{1}, j_{2} \leqslant n,
$$

where $F^{j_{1}, j_{2}}=F^{j_{2}, j_{1}}$. Under a local change of coordinates $(x, y) \mapsto(X, Y)=$ $(X(x, y), Y(x, y))$, this system (1.8) is equivalent to the simplest "flat" system

$$
Y_{X^{j_{1}} X^{j_{2}}}=0, \quad 1 \leqslant j_{1}, j_{2} \leqslant n,
$$

if and only if there exist arbitrary functions $G_{j_{1}, j_{2}}, H_{j_{1}, j_{2}}^{k_{1}}, L_{j_{1}}^{k_{1}}$ and $M^{k_{1}}$ of the variables $(x, y)$, for $1 \leqslant j_{1}, j_{2}, k_{1} \leqslant n$, satisfying the two symmetry conditions $G_{j_{1}, j_{2}}=G_{j_{2}, j_{1}}$ and $H_{j_{1}, j_{2}}^{k_{1}}=H_{j_{2}, j_{1}}^{k_{1}}$, such that the equation (1.8) is of the specific cubic polynomial form:

$$
y_{x^{j_{1}} x^{j_{2}}}=G_{j_{1}, j_{2}}+\sum_{k_{1}=1}^{n} y_{x^{k_{1}}}\left(H_{j_{1}, j_{2}}^{k_{1}}+\frac{1}{2} y_{x^{j_{1}}} L_{j_{2}}^{k_{1}}+\frac{1}{2} y_{x^{j_{2}}} L_{j_{1}}^{k_{1}}+y_{x^{j_{1}}} y_{x^{j_{2}}} M^{k_{1}}\right)
$$

for $j_{1}, j_{2}=1, \ldots, n$.

It may seem quite paradoxical and counter-intuitive (or even false?) that every system (1.10), for arbitrary choices of functions $G_{j_{1}, j_{2}}, H_{j_{1}, j_{2}}^{k_{1}}, L_{j_{1}}^{k_{1}}$ and $M^{k_{1}}$, is automatically equivalent to $Y_{X^{j_{1}} X^{j_{2}}}=0$. However, a strong hidden assumption holds: that of complete integrability. Shortly, this crucial condition amounts to say that

$$
D_{j_{3}}\left(F^{j_{1}, j_{2}}\right)=D_{j_{2}}\left(F^{j_{1}, j_{3}}\right)
$$

for all $j_{1}, j_{2}, j_{3}=1, \ldots, n$, where, for $j=1, \ldots, n$, the $D_{j}$ are the total differentiation operators defined by

$$
D_{j}:=\frac{\partial}{\partial x^{j}}+y_{x^{j}} \frac{\partial}{\partial y}+\sum_{l=1}^{n} F^{j, l} \frac{\partial}{\partial y_{x^{l}}} .
$$


These conditions are non-void precisely when $n \geqslant 2$. More concretely, developing out (1.11) when the $F^{j_{1}, j_{2}}$ are of the specific cubic polynomial form (1.10), after some nontrivial manual computation, we obtain the complicated cubic differential polynomial in the variables $y_{x^{k}}$. Equating to zero all the coefficients of this cubic polynomial, we obtain four familes (I'), (II'), (III') and (IV') of first order partial differential equations satisfied by $G_{j_{1}, j_{2}}, H_{j_{1}, j_{2}}^{k_{1}}, L_{j_{1}}^{k_{1}}$ and $M^{k_{1}}$ :

(I')

$$
\left\{0=G_{j_{1}, j_{2}, x^{j_{3}}}-G_{j_{1}, j_{3}, x^{j_{2}}}+\sum_{k_{1}=1}^{n} H_{j_{1}, j_{2}}^{k_{1}} G_{k_{1}, j_{3}}-\sum_{k_{1}=1}^{n} H_{j_{1}, j_{3}}^{k_{1}} G_{k_{1}, j_{2}} .\right.
$$

(II')

$$
\left\{\begin{aligned}
0= & \delta_{j_{3}}^{k_{1}} G_{j_{1}, j_{2}, y}-\delta_{j_{2}}^{k_{1}} G_{j_{1}, j_{3}, y}+H_{j_{1}, j_{2}, x^{j_{3}}}^{k_{1}}-H_{j_{1}, j_{3}, x^{j_{2}}}^{k_{1}}+ \\
& +\frac{1}{2} G_{j_{1}, j_{3}} L_{j_{2}}^{k_{1}}-\frac{1}{2} G_{j_{1}, j_{2}} L_{j_{3}}^{k_{1}}+ \\
& +\frac{1}{2} \delta_{j_{1}}^{k_{1}} \sum_{k_{2}=1}^{n} G_{k_{2}, j_{3}} L_{j_{2}}^{k_{2}}-\frac{1}{2} \delta_{j_{1}}^{k_{1}} \sum_{k_{2}=1}^{n} G_{k_{2}, j_{2}} L_{j_{3}}^{k_{2}}+ \\
& +\frac{1}{2} \delta_{j_{2}}^{k_{1}} \sum_{k_{2}=1}^{n} G_{k_{2}, j_{3}} L_{j_{1}}^{k_{2}}-\frac{1}{2} \delta_{j_{3}}^{k_{1}} \sum_{k_{2}=1}^{n} G_{k_{2}, j_{2}} L_{j_{1}}^{k_{2}}+ \\
& +\sum_{k_{2}=1}^{n} H_{k_{2}, j_{3}}^{k_{1}} H_{j_{1}, j_{2}}^{k_{2}}-\sum_{k_{2}=1}^{n} H_{k_{2}, j_{2}}^{k_{1}} H_{j_{1}, j_{3}}^{k_{2}} .
\end{aligned}\right.
$$

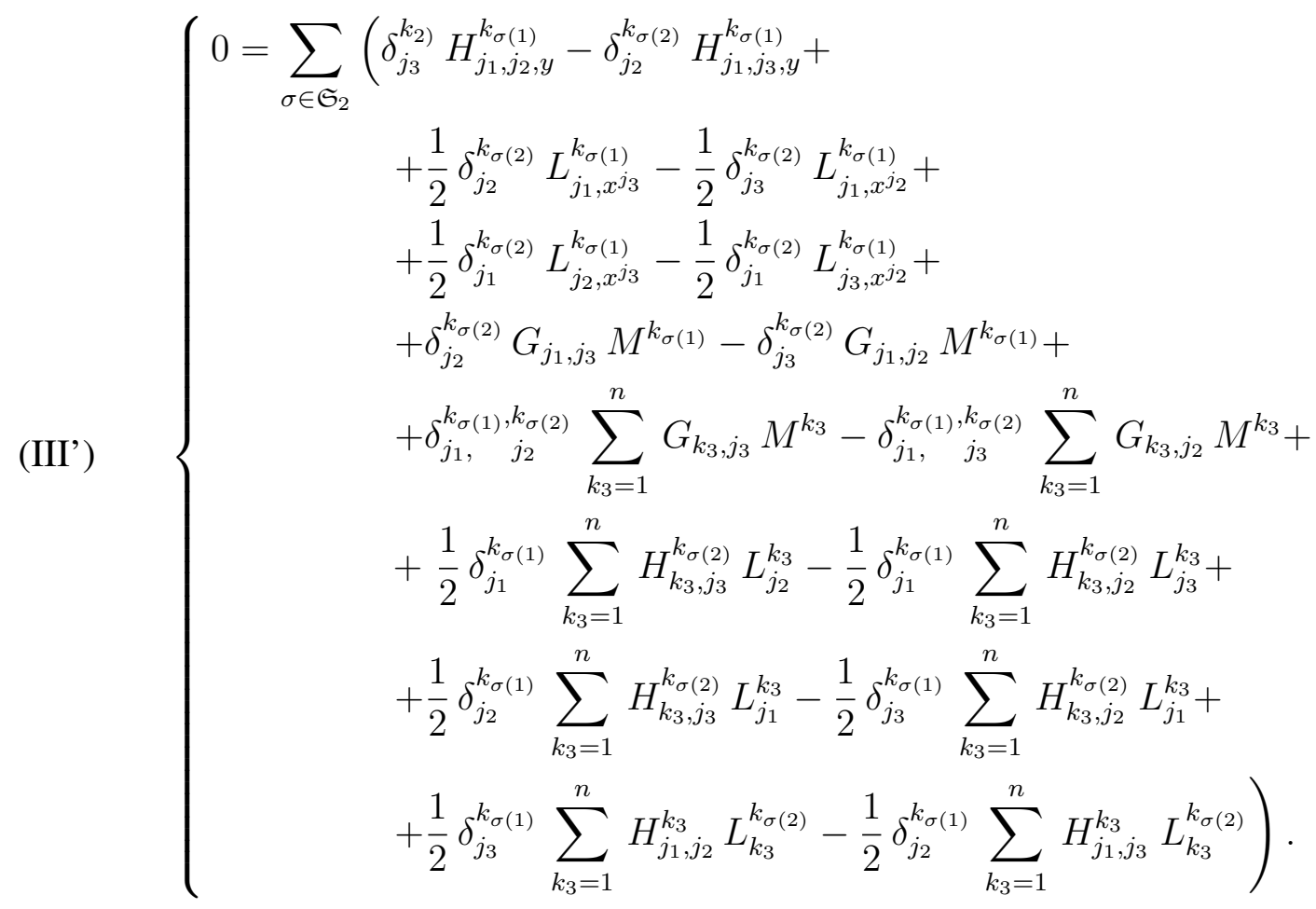


$(\mathrm{IV})$

$$
\left\{\begin{aligned}
0=\sum_{\sigma \in \mathfrak{S}_{3}} & \left(\frac{1}{2} \delta_{j_{3}, \quad j_{1}}^{k_{\sigma(3)}, k_{\sigma(2)}} L_{j_{2}, y}^{k_{\sigma(1)}}-\frac{1}{2} \delta_{j_{2}, \quad j_{1}}^{k_{\sigma(3)}, k_{\sigma(2)}} L_{j_{3}, y}^{k_{\sigma(1)}}+\right. \\
& +\delta_{j_{2}, \quad j_{1}}^{k_{\sigma(3)}, k_{\sigma(2)}} M_{x^{j_{3}}}^{k_{\sigma(1)}}-\delta_{j_{3}, \quad j_{1}}^{k_{\sigma(3)}, k_{\sigma(2)}} M_{x^{j_{2}}}^{k_{j(1)}}+ \\
& +\delta_{j_{2}, \quad j_{1}}^{k_{\sigma(3)}, k_{\sigma(1)}} \sum_{k_{4}=1}^{n} H_{k_{4}, j_{3}}^{k_{\sigma(2)}} M^{k_{4}}-\delta_{j_{3}, \quad j_{1}}^{k_{\sigma(3)}, k_{\sigma(1)}} \sum_{k_{4}=1}^{n} H_{k_{4}, j_{2}}^{k_{\sigma(2)}} M^{k_{4}}+ \\
& \left.+\frac{1}{4} \delta_{j_{1}, \quad j_{3}}^{k_{\sigma(1)}, k_{\sigma(3)}} \sum_{k_{4}=1}^{n} L_{k_{4}}^{k_{\sigma(2)}} L_{j_{2}}^{k_{4}}-\frac{1}{4} \delta_{j_{1}, \quad j_{2}}^{k_{\sigma(1)}, k_{\sigma(3)}} \sum_{k_{4}=1}^{n} L_{k_{4}}^{k_{\sigma(2)}} L_{j_{3}}^{k_{4}}\right) .
\end{aligned}\right.
$$

(These systems (I'), (II'), (III') and (IV') should be distinguished from the systems (I), (II), (III) and (IV) of Theorem 1.7 in [Me2004], although they are quite similar.) Here, the indices $j_{1}, j_{2}, j_{3}, k_{1}, k_{2}, k_{3}$ vary in $\{1,2, \ldots, n\}$. By $\mathfrak{S}_{2}$ and by $\mathfrak{S}_{3}$, we denote the permutation group of $\{1,2\}$ and of $\{1,2,3\}$. To facilitate hand- and Latex-writing, partial derivatives are denoted as indices after a comma; for instance, $G_{j_{1}, j_{2}, x^{j_{3}}}$ is an abreviation for $\partial G_{j_{1}, j_{2}} / \partial x^{j_{3}}$. To deduce (I'), (II'), (III') and (IV') from equation (1.11), we use the fact that every cubic polynomial equation of the form

$$
\begin{aligned}
0 \equiv A & +\sum_{k_{1}=1}^{n} B_{k_{1}} \cdot y_{x^{k_{1}}}+\sum_{k_{1}=1}^{n} \sum_{k_{2}=1}^{n} C_{k_{1}, k_{2}} \cdot y_{x^{k_{1}}} y_{x^{k_{2}}}+ \\
& +\sum_{k_{1}=1}^{n} \sum_{k_{2}=1}^{n} \sum_{k_{2}=1}^{n} D_{k_{1}, k_{2}, k_{3}} \cdot y_{x^{k_{1}}} y_{x^{k_{2}}} y_{x^{k_{3}}}
\end{aligned}
$$

is equivalent to the annihilation of the following symmetric sums of its coefficients:

$$
\left\{\begin{array}{l}
0=A \\
0=B_{k_{1}} \\
0=C_{k_{1}, k_{2}}+C_{k_{2}, k_{1}} \\
0=D_{k_{1}, k_{2}, k_{3}}+D_{k_{3}, k_{1}, k_{2}}+D_{k_{2}, k_{3}, k_{1}}+D_{k_{2}, k_{1}, k_{3}}+D_{k_{3}, k_{2}, k_{1}}+D_{k_{1}, k_{3}, k_{2}}
\end{array}\right.
$$

for all $k_{1}, k_{2}, k_{3}=1, \ldots, n$.

In conclusion, the functions $G_{j_{1}, j_{2}}, H_{j_{1}, j_{2}}^{k_{1}}, L_{j_{1}}^{k_{1}}$ and $M^{k_{1}}$ in the statement of Theorem 1.7 are far from being arbitrary: they satisfy the complicated system of first order partial differential equations (I'), (II'), (III') and (IV') above.

Our proof of Theorem 1.7 is similar to the one provided in [Me2004], in the case of systems of second order ordinary differential equations, so that most steps of the proof will be summarized.

In the end of this paper, we will delineate a complicated system of second order partial differential equations satisfied by $G_{j_{1}, j_{2}}, H_{j_{1}, j_{2}}^{k_{1}}, L_{j_{1}}^{k_{1}}$ and $M^{k_{1}}$ which is the exact analog of the system described in the abstract. The main technical part of the proof of Theorem 1.7 will be to establish that this second order system is a consequence, by linear combinations and by differentiations, of the first order system (I'), (II'), (III') and (IV').

Open question 1.15. Are Theorems 1.1 and 1.7 true under weaker smoothness assumptions, namely with a $\mathcal{C}^{2}$ or a $W_{\mathrm{loc}}^{1, \infty}$ right-hand side?

We refer to [Ma2003] for inspiration and appropriate tools.

Open question 1.16. Deduce from Theorem 1.7 an explicit necessary and sufficient condition for the associated submanifold of solutions $y=b+\Pi\left(x^{i}, a^{k}, b\right)$ to be locally equivalent to $Y=B+X^{1} A^{1}+\cdots+X^{n} A^{n}$. 
As an application, this would characterize local sphericity of a Levi nondegenerate hypersurface $M \subset \mathbb{C}^{n+1}$ with $n \geqslant 2$.

Generalizing the Lie-Tresse classification would be a great achievement.

Open problem 1.17. For $n=2$ establish a complete list of normal forms of all possible systems (1.?) according to their Lie symmetry group. In case of success, classify Levi nondegenerate real analytic hypersurfaces of $\mathbb{C}^{3}$ up to biholomorphisms.

\section{$\S 2$. COMPLETELY INTEGRABLE SYSTEMS OF SECOND ORDER ORDINARY DIFFERENTIAL EQUATIONS}

2.1. Prolongation of a point transformation to the second order jet space. Let $\mathbb{K}=\mathbb{R}$ or $\mathbb{C}$, let $n \in \mathbb{N}$, suppose $n \geqslant 2$, let $x=\left(x^{1}, \ldots, x^{n}\right) \in \mathbb{K}^{n}$ and let $y \in \mathbb{K}$. According to the main assumption of Theorem 1.7 , we have to consider a local $\mathbb{K}$-analytic diffeomorphism of the form

$$
\left(x^{j_{1}}, y\right) \longmapsto\left(X^{j}\left(x^{j_{1}}, y\right), Y\left(x^{j_{1}}, y\right)\right),
$$

which transforms the system (1.8) to the system $Y_{X^{i_{1}} X^{i_{2}}}=0,1 \leqslant j_{1}, j_{2} \leqslant n$. Without loss of generality, we shall assume that this transformation is close to the identity. To obtain the precise expression (2.35) of the transformed system (1.8), we have to prolong the above diffeomorphism to the second order jet space. We introduce the coordinates $\left(x^{j}, y, y_{x^{j_{1}}}, y_{x^{j_{1}} x^{j_{2}}}\right)$ on the second order jet space. Let

$$
D_{k}:=\frac{\partial}{\partial x^{k}}+y_{x^{k}} \frac{\partial}{\partial y}+\sum_{l=1}^{n} y_{x^{k} x^{l}} \frac{\partial}{\partial y_{x^{l}}},
$$

be the $k$-th total differentiation operator. According to [O11986, BK1989, O11995], for the first order partial derivatives, one has the (implicit, compact) expression:

$$
\left(\begin{array}{c}
Y_{X^{1}} \\
\vdots \\
Y_{X^{n}}
\end{array}\right)=\left(\begin{array}{ccc}
D_{1} X^{1} & \cdots & D_{1} X^{n} \\
\vdots & \cdots & \vdots \\
D_{n} X^{1} & \cdots & D_{n} X^{n}
\end{array}\right)^{-1}\left(\begin{array}{c}
D_{1} Y \\
\vdots \\
D_{n} Y
\end{array}\right)
$$

where $(\cdot)^{-1}$ denotes the inverse matrix, which exists, since the transformation (2.2) is close to the identity. For the second order partial derivatives, again according to [O11986, BK1989, Ol1995], one has the (implicit, compact) expressions:

$$
\left(\begin{array}{c}
Y_{X^{j} X^{1}} \\
\vdots \\
Y_{X^{j} X^{n}}
\end{array}\right)=\left(\begin{array}{ccc}
D_{1} X^{1} & \cdots & D_{1} X^{n} \\
\vdots & \cdots & \vdots \\
D_{n} X^{1} & \cdots & D_{n} X^{n}
\end{array}\right)^{-1}\left(\begin{array}{c}
D_{1} Y_{X^{j}} \\
\vdots \\
D_{n} Y_{X^{j}}
\end{array}\right)
$$

for $j=1, \ldots, n$. Let $D X$ denote the matrix $\left(D_{i} X^{j}\right)_{1 \leqslant i \leqslant n}^{1 \leqslant j \leqslant n}$, where $i$ is the index of lines and $j$ the index of columns, let $Y_{X}$ denote the column matrix $\left(Y_{X^{i}}\right)_{1 \leqslant i \leqslant n}$ and let $D Y$ be the column matrix $\left(D_{i} Y\right)_{1 \leqslant i \leqslant n}$.

By inspecting (2.5) above, we see that the equivalence between (i), (ii) and (iii) just below is obvious:

Lemma 2.6. The following conditions are equivalent:

(i) the differential equations $Y_{X^{j} X^{k}}=0$ hold for $1 \leqslant j, k \leqslant n$;

(ii) the matrix equations $D_{k}\left(Y_{X}\right)=0$ hold for $1 \leqslant k \leqslant n$;

(iii) the matrix equations $D X \cdot D_{k}\left(Y_{X}\right)=0$ hold for $1 \leqslant k \leqslant n$;

(iv) the matrix equations $0=D_{k}(D X) \cdot Y_{X}-D_{k}(D Y)$ hold for $1 \leqslant k \leqslant n$. 
Formally, in the sequel, it will be more convenient to achieve the explicit computations starting from condition (iv), since no matrix inversion at all is involved in it.

Proof. Indeed, applying the total differentiation operator $D_{k}$ to the matrix equation (2.4) written under the equivalent form $0=D X \cdot Y_{X}-D Y$, we get:

$$
0=D_{k}(D X) \cdot Y_{X}+D X \cdot D_{k}\left(Y_{X}\right)-D_{k}(D Y),
$$

so that the equivalence between (iii) and (iv) is now clear.

2.8. An explicit formula in the case $n=2$. Thus, we can start to develope explicitely the matrix equations

$$
0=D_{k}(D X) \cdot Y_{X}-D_{k}(D Y)
$$

In it, some huge formal expressions are hidden behind the symbol $D_{k}$. Proceeding inductively, we start by examinating the case $n=2$ thoroughly. By direct computations which require to be clever, we reconstitute some $3 \times 3$ determinants in the four (in fact three) developed equations (2.9). After some work, the first equation is:

$$
\begin{aligned}
& 0=y_{x^{1} x^{1}} \cdot\left|\begin{array}{ccc}
X_{x^{1}}^{1} & X_{x^{2}}^{1} & X_{y}^{1} \\
X_{x^{1}}^{2} & X_{x^{2}}^{2} & X_{y}^{2} \\
Y_{x^{1}} & Y_{x^{2}} & Y_{y}
\end{array}\right|+\left|\begin{array}{ccc}
X_{x^{1}}^{1} & X_{x^{2}}^{1} & X_{x^{1} x^{1}}^{1} \\
X_{x^{1}}^{2} & X_{x^{2}}^{2} & X_{x^{1} x^{1}}^{2} \\
Y_{x^{1}} & Y_{x^{2}} & Y_{x^{1} x^{1}}
\end{array}\right|+ \\
& +y_{x^{1}} \cdot\left\{2\left|\begin{array}{ccc}
X_{x^{1}}^{1} & X_{x^{2}}^{1} & X_{x^{1} y}^{1} \\
X_{x^{1}}^{2} & X_{x^{2}}^{2} & X_{x^{1} y}^{2} \\
Y_{x^{1}} & Y_{x^{2}} & Y_{x^{1} y}
\end{array}\right|-\left|\begin{array}{ccc}
X_{x^{1} x^{1}}^{1} & X_{x^{2}}^{1} & X_{y}^{1} \\
X_{x^{1} x^{1}} & X_{x^{2}}^{2} & X_{y}^{2} \\
Y_{x^{1} x^{1}} & Y_{x^{2}} & Y_{y}
\end{array}\right|\right\}+ \\
& +y_{x^{2}} \cdot\left\{-\left|\begin{array}{ccc}
X_{x^{1}}^{1} & X_{x^{1} x^{1}}^{1} & X_{y}^{1} \\
X_{x^{1}}^{2} & X_{x^{1} x^{1}}^{2} & X_{y}^{2} \\
Y_{x^{1}} & Y_{x^{1} x^{1}} & Y_{y}
\end{array}\right|\right\}+ \\
& +y_{x^{1}} y_{x^{1}} \cdot\left\{\left|\begin{array}{ccc}
X_{x^{1}}^{1} & X_{x^{2}}^{1} & X_{y y}^{1} \\
X_{x^{1}}^{2} & X_{x^{2}}^{2} & X_{y y}^{2} \\
Y_{x^{1}} & Y_{x^{2}} & Y_{y y}
\end{array}\right|-2\left|\begin{array}{ccc}
X_{x^{1} y}^{1} & X_{x^{2}}^{1} & X_{y}^{1} \\
X_{x^{1} y}^{2} & X_{x^{2}}^{2} & X_{y}^{2} \\
Y_{x^{1} y} & Y_{x^{2}} & Y_{y}
\end{array}\right|\right\}+ \\
& +y_{x^{1}} y_{x^{2}} \cdot\left\{-2\left|\begin{array}{ccc}
X_{x^{1}}^{1} & X_{x^{1} y}^{1} & X_{y}^{1} \\
X_{x^{1}}^{2} & X_{x^{1} y}^{2} & X_{y}^{2} \\
Y_{x^{1}} & Y_{x^{1} y} & Y_{y}
\end{array}\right|\right\}+ \\
& +y_{x^{1}} y_{x^{1}} y_{x^{1}} \cdot\left\{-\left|\begin{array}{ccc}
X_{y y}^{1} & X_{x^{2}}^{1} & X_{y}^{1} \\
X_{y y}^{2} & X_{x^{2}}^{2} & X_{y}^{2} \\
Y_{y y} & Y_{x^{2}} & Y_{y}
\end{array}\right|\right\}+ \\
& +y_{x^{1}} y_{x^{1}} y_{x^{2}} \cdot\left\{-\left|\begin{array}{ccc}
X_{x^{1}}^{1} & X_{y y}^{1} & X_{y}^{1} \\
X_{x^{1}}^{2} & X_{y y}^{2} & X_{y}^{2} \\
Y_{x^{1}} & Y_{y y} & Y_{y}
\end{array}\right|\right\}
\end{aligned}
$$

This formula and the two next (2.22), (2.23) have been checked by Sylvain Neut and Michel Petitot with the help of Maple.

2.11. Comparison with the coefficients of the second prolongation of a vector field. At present, it is useful to make an illuminating digression which will help us to devise what is the general form of the development of the equations (2.9). Consider an arbitrary vector field of the form

$$
\mathcal{L}:=\sum_{k=1}^{n} \mathcal{X}^{k} \frac{\partial}{\partial x^{k}}+\mathcal{Y} \frac{\partial}{\partial y}
$$


where the coefficients $\mathcal{X}^{k}$ and $\mathcal{Y}$ are functions of $\left(x^{i}, y\right)$. According to [O11986, BK1989, O11995], there exists a unique prolongation $\mathcal{L}^{(2)}$ of this vector field to the second order jet space, of the form

$$
\mathcal{L}^{(2)}:=\mathcal{L}+\sum_{j_{1}=1}^{n} \mathbf{Y}_{j_{1}} \frac{\partial}{\partial y_{x^{j_{1}}}}+\sum_{j_{1}=1}^{n} \sum_{j_{2}=1}^{n} \mathbf{Y}_{j_{1}, j_{2}} \frac{\partial}{\partial y_{x^{j_{1}} x^{j_{2}}}},
$$

where the coefficients $\mathbf{Y}_{j_{1}}, \mathbf{Y}_{j_{1}, j_{2}}$ may be computed by means of formulas (3.4) of Section 3(II). In Part II, we obtained the following perfect formulas:

$$
\left\{\begin{aligned}
\mathbf{Y}_{j_{1}, j_{2}}= & \mathcal{Y}_{x^{j_{1}} x^{j_{2}}}+\sum_{k_{1}=1}^{n} y_{x^{k_{1}}} \cdot\left\{\delta_{j_{1}}^{k_{1}} \mathcal{Y}_{x^{j_{2} y}}+\delta_{j_{2}}^{k_{1}} \mathcal{Y}_{x^{j_{1}} y}-\mathcal{X}_{x^{j_{1}} x^{j_{2}}}^{k_{1}}\right\}+ \\
& +\sum_{k_{1}=1}^{n} \sum_{k_{2}=1}^{n} y_{x^{k_{1}}} y_{x^{k_{2}}} \cdot\left\{\delta_{j_{1}, j_{2}}^{k_{1}, k_{2}} \mathcal{Y}_{y y}-\delta_{j_{1}}^{k_{1}} \mathcal{X}_{x^{j_{2}} y}^{k_{2}}-\delta_{j_{2}}^{k_{1}} \mathcal{X}_{x^{j_{1} y}}^{k_{2}}\right\}+ \\
& +\sum_{k_{1}=1}^{n} \sum_{k_{2}=1}^{n} \sum_{k_{3}=1}^{n} y_{x^{k_{1}}} y_{x^{k_{2}}} y_{x^{k_{3}}} \cdot\left\{-\delta_{j_{1}, j_{2}}^{k_{1}, k_{2}} \mathcal{X}_{y y}^{k_{3}}\right\}
\end{aligned}\right.
$$

for $j_{1}, j_{2}=1, \ldots, n$. The expression of $\mathbf{Y}_{j_{1}}$ does not matter for us here. Specifying this formula to the the case $n=2$ and taking account of the symmetry $\mathbf{Y}_{1,2}=\mathbf{Y}_{2,1}$ we get the following three second order coefficients:

$$
\left\{\begin{aligned}
\mathbf{Y}_{1,1}= & \mathcal{Y}_{x^{1} x^{1}}+y_{x^{1}} \cdot\left\{2 \mathcal{Y}_{x^{1} y}-\mathcal{X}_{x^{1} x^{1}}^{1}\right\}+y_{x^{2}} \cdot\left\{-\mathcal{X}_{x^{1} x^{1}}^{2}\right\}+ \\
& +y_{x^{1}} y_{x^{1}} \cdot\left\{\mathcal{Y}_{y y}-2 \mathcal{X}_{x^{1} y}^{1}\right\}+y_{x^{1}} y_{x^{2}} \cdot\left\{-2 \mathcal{X}_{x^{1} y}^{2}\right\}+ \\
& +y_{x^{1}} y_{x^{1}} y_{x^{1}} \cdot\left\{-\mathcal{X}_{y y}^{1}\right\}+y_{x^{1}} y_{x^{1}} y_{x^{2}} \cdot\left\{-\mathcal{X}_{y y}^{2}\right\} \\
\mathbf{Y}_{1,2}= & \mathcal{Y}_{x^{1} x^{2}}+y_{x^{1}} \cdot\left\{\mathcal{Y}_{x^{2} y}-\mathcal{X}_{x^{1} x^{2}}^{1}\right\}+y_{x^{2}} \cdot\left\{\mathcal{Y}_{x^{1} y}-\mathcal{X}_{x^{1} x^{2}}^{2}\right\}+ \\
& +y_{x^{1}} y_{x^{1}} \cdot\left\{-\mathcal{X}_{x^{2} y}^{1}\right\}+y_{x^{1}} y_{x^{2}} \cdot\left\{\mathcal{Y}_{y y}-\mathcal{X}_{x^{1} y}^{1}-\mathcal{X}_{x^{2} y}^{2}\right\}+ \\
& +y_{x^{2}} y_{x^{2}} \cdot\left\{-\mathcal{X}_{x^{1} y}^{2}\right\}+ \\
& +y_{x^{1}} y_{x^{1}} y_{x^{2}} \cdot\left\{-\mathcal{X}_{y y}^{1}\right\}+y_{x^{1}} y_{x^{2}} y_{x^{2}} \cdot\left\{-\mathcal{X}_{y y}^{2}\right\} \\
\mathbf{Y}_{2,2}= & \mathcal{Y}_{x^{2} x^{2}}+y_{x^{1}} \cdot\left\{-\mathcal{X}_{x^{2} x^{2}}^{1}\right\}+y_{x^{2}} \cdot\left\{2 \mathcal{Y}_{x^{2} y}-\mathcal{X}_{x^{2} x^{2}}^{2}\right\}+ \\
& +y_{x^{1}} y_{x^{2}} \cdot\left\{-2 \mathcal{X}_{x^{2} y}^{1}\right\}+y_{x^{2}} y_{x^{2}} \cdot\left\{\mathcal{Y}_{y y}-2 \mathcal{X}_{x^{2} y}^{2}\right\}+ \\
& +y_{x^{1}} y_{x^{2}} y_{x^{2}} \cdot\left\{-\mathcal{X}_{y y}^{1}\right\}+y_{x^{2}} y_{x^{2}} y_{x^{2}} \cdot\left\{-\mathcal{X}_{y y}^{2}\right\}
\end{aligned}\right.
$$

We would like to mention that the computation of $\mathbf{Y}_{j_{1}, j_{2}}, 1 \leqslant j_{1}, j_{2} \leqslant 2$, above is easier than the verification of (2.10). Based on the three formulas (2.15), we claim that we can guess the second and the third equations, which would be obtained by developing and by simplifying (2.9), namely with $y_{x^{1} x^{2}}$ and with $y_{x^{2} x^{2}}$ instead of $y_{x^{1} x^{2}}$ in (2.10). Our dictionary to translate from the first formula (2.15) to (2.10) may be described as follows. Begin with the Jacobian determinant

$$
\left|\begin{array}{ccc}
X_{x^{1}}^{1} & X_{x^{2}}^{1} & X_{y}^{1} \\
X_{x^{1}}^{2} & X_{x^{2}}^{2} & X_{y}^{2} \\
Y_{x^{1}} & Y_{x^{2}} & Y_{y}
\end{array}\right|
$$

of the change of coordinates (2.2). Since this change of coordinates is close to the identity, we may consider that the following Jacobian matrix approximation holds:

$$
\left(\begin{array}{ccc}
X_{x^{1}}^{1} & X_{x^{2}}^{1} & X_{y}^{1} \\
X_{x^{1}}^{2} & X_{x^{2}}^{2} & X_{y}^{2} \\
Y_{x^{1}} & Y_{x^{2}} & Y_{y}
\end{array}\right) \cong\left(\begin{array}{ccc}
1 & 0 & 0 \\
0 & 1 & 0 \\
0 & 0 & 1
\end{array}\right)
$$


The jacobian matrix has three columns. There are six possible second order derivatives with respect to the variables $\left(x^{1}, x^{2}, y\right)$, namely

$$
(\cdot)_{x^{1} x^{1}}, \quad(\cdot)_{x^{1} x^{2}}, \quad(\cdot)_{x^{2} x^{2}}, \quad(\cdot)_{x^{1} y}, \quad(\cdot)_{x^{2} y}, \quad(\cdot)_{y y} \cdot
$$

In the Jacobian determinant (2.16), by replacing any one of the three columns of first order derivatives with a column of second order derivatives, we obtain exactly $3 \times 6=18$ possible determinants. For instance, by replacing the third column by the second order derivative $(\cdot)_{x^{1} y}$ or the first column by the second order derivative $(\cdot)_{x^{1} x^{1}}$, we get:

$$
\left|\begin{array}{ccc}
X_{x^{1}}^{1} & X_{x^{2}}^{1} & X_{x^{1} y}^{1} \\
X_{x^{1}}^{2} & X_{x^{2}}^{2} & X_{x^{1} y}^{2} \\
Y_{x^{1}} & Y_{x^{2}} & Y_{x^{1} y}
\end{array}\right| \quad \text { or } \quad\left|\begin{array}{ccc}
X_{x^{1} x^{1}}^{1} & X_{x^{2}}^{1} & X_{y}^{1} \\
X_{x^{1} x^{1}}^{2} & X_{x^{2}}^{2} & X_{y}^{2} \\
Y_{x^{1} x^{1}} & Y_{x^{2}} & Y_{y}
\end{array}\right|
$$

We recover the two determinants appearing in the second line of (2.10). On the other hand, according to the approximation (2.17), these two determinants are essentially equal to

$$
\left|\begin{array}{ccc}
1 & 0 & X_{x^{1} y}^{1} \\
0 & 1 & X_{x^{1} y}^{2} \\
0 & 0 & Y_{x^{1} y}^{1}
\end{array}\right|=Y_{x^{1} y} \quad \text { or to } \quad\left|\begin{array}{ccc}
X_{x^{1} x^{1}}^{1} & 0 & 0 \\
X_{x^{1} x^{1}}^{2} & 1 & 0 \\
Y_{x^{1} x^{1}} & 0 & 1
\end{array}\right|=X_{x^{1} x^{1}}^{1}
$$

Consequently, in the second line of (2.10), up to a change to calligraphic letters, we recover the coefficient

$$
2 \mathcal{Y}_{x^{1} y}-\mathcal{X}_{x^{1} x^{1}}^{1}
$$

of $y_{x_{1}}$ in the expression of $\mathbf{Y}_{1,1}$ in (2.15). In conclusion, we have discovered how to pass symbolically from the first equation (2.15) to the equation (2.10) and conversely.

Translating the second equation (2.15), we deduce, without any further computation, that the second equation which would be obtained by developing (2.9) in length, is:

$$
\begin{aligned}
0=y_{x^{1} x^{2}} \cdot & \left|\begin{array}{ccc}
X_{x^{1}}^{1} & X_{x^{2}}^{1} & X_{y}^{1} \\
X_{x^{1}}^{2} & X_{x^{2}}^{2} & X_{y}^{2} \\
Y_{x^{1}} & Y_{x^{2}} & Y_{y}
\end{array}\right|+\left|\begin{array}{ccc}
X_{x^{1}}^{1} & X_{x^{2}}^{1} & X_{x^{1} x^{2}}^{1} \\
X_{x^{1}}^{2} & X_{x^{2}}^{2} & X_{x^{1} x^{2}}^{2} \\
Y_{x^{1}} & Y_{x^{2}} & Y_{x^{1} x^{2}}
\end{array}\right|+ \\
+y_{x^{1}} \cdot & \left\{\left|\begin{array}{ccc}
X_{x^{1}}^{1} & X_{x^{2}}^{1} & X_{x^{2} y}^{1} \\
X_{x^{1}}^{2} & X_{x^{2}}^{2} & X_{x^{2} y}^{2} \\
Y_{x^{1}} & Y_{x^{2}} & Y_{x^{2} y}
\end{array}\right|-\left|\begin{array}{ccc}
X_{x^{1} x^{2}}^{1} & X_{x^{2}}^{1} & X_{y}^{1} \\
X_{x^{1} x^{2}}^{2} & X_{x^{2}}^{2} & X_{y}^{2} \\
Y_{x^{1} x^{2}} & Y_{x^{2}} & Y_{y}
\end{array}\right|\right\}+ \\
+y_{x^{2}} \cdot & \left\{\begin{array}{ccc}
X_{x^{1}}^{1} & X_{x^{2}}^{1} & X_{x^{1} y}^{1} \\
X_{x^{1}}^{2} & X_{x^{2}}^{2} & X_{x^{1} y}^{2} \\
Y_{x^{1}} & Y_{x^{2}} & Y_{x^{1} y}
\end{array}|-| \begin{array}{ccc}
X_{x^{1}}^{1} & X_{x^{1} x^{2}}^{1} & X_{y}^{1} \\
X_{x^{1}}^{2} & X_{x^{1} x^{2}}^{2} & X_{y}^{2} \\
Y_{x^{1}} & Y_{x^{1} x^{2}} & Y_{y}
\end{array} \mid\right\}+
\end{aligned}
$$




$$
\begin{aligned}
& +y_{x^{1}} y_{x^{1}} \cdot\left\{-\left|\begin{array}{ccc}
X_{x^{2} y}^{1} & X_{x^{2}}^{1} & X_{y}^{1} \\
X_{x^{2} y}^{2} & X_{x^{2}}^{2} & X_{y}^{2} \\
Y_{x^{2} y} & Y_{x^{2}} & Y_{y}
\end{array}\right|\right\}+ \\
& +y_{x^{1}} y_{x^{2}} \cdot\left\{\begin{array}{ccc}
X_{x^{1}}^{1} & X_{x^{2}}^{1} & X_{y y}^{1} \\
X_{x^{1}}^{2} & X_{x^{2}}^{2} & X_{y y}^{2} \\
Y_{x^{1}} & Y_{x^{2}} & Y_{y y}
\end{array}|-| \begin{array}{ccc}
X_{x^{1} y}^{1} & X_{x^{2}}^{1} & X_{y}^{1} \\
X_{x^{1} y}^{2} & X_{x^{2}}^{2} & X_{y}^{2} \\
Y_{x^{1} y} & Y_{x^{2}} & Y_{y}
\end{array} \mid-\right. \\
& \left.-\left|\begin{array}{ccc}
X_{x^{1}}^{1} & X_{x^{2} y}^{1} & X_{y}^{1} \\
X_{x^{1}}^{2} & X_{x^{2} y}^{2} & X_{y}^{2} \\
Y_{x^{1}} & Y_{x^{2} y} & Y_{y}
\end{array}\right|\right\}+y_{x^{2}} y_{x^{2}}\left\{-\left|\begin{array}{ccc}
X_{x^{1}}^{1} & X_{x^{1} y}^{1} & X_{y}^{1} \\
X_{x^{1}}^{2} & X_{x^{1} y}^{2} & X_{y}^{2} \\
Y_{x^{1}} & Y_{x^{1} y} & Y_{y}
\end{array}\right|\right\}+ \\
& +y_{x^{1}} y_{x^{1}} y_{x^{2}} \cdot\left\{-\left|\begin{array}{ccc}
X_{y y}^{1} & X_{x^{2}}^{1} & X_{y}^{1} \\
X_{y y}^{2} & X_{x^{2}}^{2} & X_{y}^{2} \\
Y_{y y} & Y_{x^{2}} & Y_{y}
\end{array}\right|\right\}+ \\
& +y_{x^{1}} y_{x^{2}} y_{x^{2}} \cdot\left\{-\left|\begin{array}{ccc}
X_{x^{1}}^{1} & X_{y y}^{1} & X_{y}^{1} \\
X_{x^{1}}^{2} & X_{y y}^{2} & X_{y}^{2} \\
Y_{x^{1}} & Y_{y y} & Y_{y}
\end{array}\right|\right\} \text {. }
\end{aligned}
$$

Using the third equation (2.15), we also deduce, without any further computation, that the third equation which would be obtained by developing (2.9) in length, is:

$$
\begin{aligned}
& 0=y_{x^{2} x^{2}} \cdot\left|\begin{array}{ccc}
X_{x^{1}}^{1} & X_{x^{2}}^{1} & X_{y}^{1} \\
X_{x^{1}}^{2} & X_{x^{2}}^{2} & X_{y}^{2} \\
Y_{x^{1}} & Y_{x^{2}} & Y_{y}
\end{array}\right|+\left|\begin{array}{ccc}
X_{x^{1}}^{1} & X_{x^{2}}^{1} & X_{x^{2} x^{2}}^{1} \\
X_{x^{1}}^{2} & X_{x^{2}}^{2} & X_{x^{2} x^{2}}^{2} \\
Y_{x^{1}} & Y_{x^{2}} & Y_{x^{2} x^{2}}
\end{array}\right|+ \\
& +y_{x^{1}} \cdot\left\{-\left|\begin{array}{ccc}
X_{x^{2} x^{2}}^{1} & X_{x^{2}}^{1} & X_{y}^{1} \\
X_{x^{1} x^{2}}^{2} & X_{x^{2}}^{2} & X_{y}^{2} \\
Y_{x^{2} x^{2}} & Y_{x^{2}} & Y_{y}
\end{array}\right|\right\}+ \\
& +y_{x^{2}} \cdot\left\{2\left|\begin{array}{ccc}
X_{x^{1}}^{1} & X_{x^{2}}^{1} & X_{x^{2} y}^{1} \\
X_{x^{1}}^{2} & X_{x^{2}}^{2} & X_{x^{2} y}^{2} \\
Y_{x^{1}} & Y_{x^{2}} & Y_{x^{2} y}
\end{array}\right|-\left|\begin{array}{ccc}
X_{x^{1}}^{1} & X_{x^{2} x^{2}}^{1} & X_{y}^{1} \\
X_{x^{1}}^{2} & X_{x^{2} x^{2}}^{2} & X_{y}^{2} \\
Y_{x^{1}} & Y_{x^{2} x^{2}} & Y_{y}
\end{array}\right|\right\}+ \\
& +y_{x^{1}} y_{x^{2}} \cdot\left\{-2\left|\begin{array}{ccc}
X_{x^{2} y}^{1} & X_{x^{2}}^{1} & X_{y}^{1} \\
X_{x^{2} y}^{2} & X_{x^{2}}^{2} & X_{y}^{2} \\
Y_{x^{2} y} & Y_{x^{2}} & Y_{y}
\end{array}\right|\right\}+ \\
& +y_{x^{2}} y_{x^{2}} \cdot\left\{\left|\begin{array}{ccc}
X_{x^{1}}^{1} & X_{x^{2}}^{1} & X_{y y}^{1} \\
X_{x^{1}}^{2} & X_{x^{2}}^{2} & X_{y y}^{2} \\
Y_{x^{1}} & Y_{x^{2}} & Y_{y y}
\end{array}\right|-2\left|\begin{array}{ccc}
X_{x^{1}}^{1} & X_{x^{2} y}^{1} & X_{y}^{1} \\
X_{x^{1}}^{2} & X_{x^{2} y}^{2} & X_{y}^{2} \\
Y_{x^{1}} & Y_{x^{2} y} & Y_{y}
\end{array}\right|\right\}+ \\
& +y_{x^{1}} y_{x^{2}} y_{x^{2}} \cdot\left\{-\left|\begin{array}{ccc}
X_{y y}^{1} & X_{x^{2}}^{1} & X_{y}^{1} \\
X_{y y}^{2} & X_{x^{2}}^{2} & X_{y}^{2} \\
Y_{y y} & Y_{x^{2}} & Y_{y}
\end{array}\right|\right\}+ \\
& +y_{x^{2}} y_{x^{2}} y_{x^{2}} \cdot\left\{-\left|\begin{array}{ccc}
X_{x^{1}}^{1} & X_{y y}^{1} & X_{y}^{1} \\
X_{x^{1}}^{2} & X_{y y}^{2} & X_{y}^{2} \\
Y_{x^{1}} & Y_{y y} & Y_{y}
\end{array}\right|\right\} \text {. }
\end{aligned}
$$

2.24. Appropriate formalism. To describe the combinatorics underlying formulas (2.10), (2.22) and (2.23), as in [Me2004], let us introduce the following notation for the Jacobian determinant:

$$
\Delta\left(x^{1}\left|x^{2}\right| y\right):=\left|\begin{array}{ccc}
X_{x^{1}}^{1} & X_{x^{2}}^{1} & X_{y}^{1} \\
X_{x^{1}}^{2} & X_{x^{2}}^{2} & X_{y}^{2} \\
Y_{x^{1}} & Y_{x^{2}} & Y_{y}
\end{array}\right|
$$


Here, in the notation $\Delta\left(x^{1}\left|x^{2}\right| y\right)$, the three spaces between the two vertical lines $\mid$ refer to the three columns of the Jacobian determinant, and the terms $x^{1}, x^{2}, y$ in $\left(x^{1}\left|x^{2}\right| y\right)$ designate the partial derivatives appearing in each column. Accordingly, in the following two examples of modified Jacobian determinants:

$$
\left\{\begin{array}{l}
\Delta\left(\underline{x^{1} x^{2}}\left|x^{2}\right| y\right):=\left|\begin{array}{ccc}
X_{x^{1} x^{2}}^{1} & X_{x^{2}}^{1} & X_{y}^{1} \\
X_{x^{1} x^{2}}^{2} & X_{x^{2}}^{2} & X_{y}^{2} \\
Y_{x^{1} x^{2}}^{2} & Y_{x^{2}} & Y_{y}
\end{array}\right| \\
\Delta\left(x^{1}\left|x^{2}\right| \underline{x^{1} y}\right):=\left|\begin{array}{ccc}
X_{x^{1}}^{1} & X_{x^{2}}^{1} & X_{x^{1} y}^{1} \\
X_{x^{1}}^{2} & X_{x^{2}}^{2} & X_{x^{1} y}^{2} \\
Y_{x^{1}} & Y_{x^{2}} & Y_{\underline{x^{1} y}}^{2}
\end{array}\right|
\end{array} \quad\right. \text { and }
$$

we simply mean which column of first order derivatives is replaced by a column of second order derivatives in the original Jacobian determinant.

As there are 6 possible second order derivatives $(\cdot)_{x^{1} x^{1}},(\cdot)_{x^{1} x^{2}},(\cdot)_{x^{1} x^{y}},(\cdot)_{x^{2} x^{2}},(\cdot)_{x^{2} y}$ and $(\cdot)_{y y}$ together with 3 columns, we obtain $3 \times 6=18$ possible modified Jacobian determinants:

$$
\left\{\begin{array}{lrl}
\Delta\left(x^{1} x^{1}\left|x^{2}\right| y\right) & \Delta\left(x^{1}\left|x^{1} x^{1}\right| y\right) & \Delta\left(x^{1}\left|x^{2}\right| x^{1} x^{1}\right) \\
\Delta\left(x^{1} x^{2}\left|x^{2}\right| y\right) & \Delta\left(x^{1}\left|x^{1} x^{2}\right| y\right) & \Delta\left(x^{1}\left|x^{2}\right| x^{1} x^{2}\right) \\
\Delta\left(x^{1} y\left|x^{2}\right| y\right) & \Delta\left(x^{1}\left|x^{1} y\right| y\right) & \Delta\left(x^{1}\left|x^{2}\right| x^{1} y\right) \\
\Delta\left(x^{2} x^{2}\left|x^{2}\right| y\right) & \Delta\left(x^{1}\left|x^{2} x^{2}\right| y\right) & \Delta\left(x^{1}\left|x^{2}\right| x^{2} x^{2}\right) \\
\Delta\left(x^{2} y\left|x^{2}\right| y\right) & \Delta\left(x^{1}\left|x^{2} y\right| y\right) & \Delta\left(x^{1}\left|x^{2}\right| x^{2} y\right) \\
\Delta\left(y y\left|x^{2}\right| y\right) & \Delta\left(x^{1}|y y| y\right) & \Delta\left(x^{1}\left|x^{2}\right| y y\right) .
\end{array}\right.
$$

Next, we observe that if we want to solve with respect to $y_{x^{1} x^{1}}$ in (2.10), with respect to $y_{x^{1} x^{2}}$ in (2.22) and with respect to $y_{x^{2} x^{2}}$ in (2.23), we have to divide by the Jacobian determinant $\Delta\left(x^{1}\left|x^{2}\right| y\right)$. Consequently, we introduce 18 new square functions as follows: (2.28)

$$
\left\{\begin{array}{lll}
\square_{x^{1} x^{1}}^{1}:=\frac{\Delta\left(x^{1} x^{1}\left|x^{2}\right| y\right)}{\Delta\left(x^{1}\left|x^{2}\right| y\right)} & \square_{x^{1} x^{2}}^{1}:=\frac{\Delta\left(x^{1} x^{2}\left|x^{2}\right| y\right)}{\Delta\left(x^{1}\left|x^{2}\right| y\right)} & \square_{x^{1} y}^{1}:=\frac{\Delta\left(x^{1} y\left|x^{2}\right| y\right)}{\Delta\left(x^{1}\left|x^{2}\right| y\right)} \\
\square_{x^{2} x^{2}}^{1}:=\frac{\Delta\left(x^{2} x^{2}\left|x^{2}\right| y\right)}{\Delta\left(x^{1}\left|x^{2}\right| y\right)} & \square_{x^{2} y}^{1}:=\frac{\Delta\left(x^{2} y\left|x^{2}\right| y\right)}{\Delta\left(x^{1}\left|x^{2}\right| y\right)} & \square_{y y}^{1}:=\frac{\Delta\left(y y\left|x^{2}\right| y\right)}{\Delta\left(x^{1}\left|x^{2}\right| y\right)} \\
\square_{x^{1} x^{1}}^{2}:=\frac{\Delta\left(x^{1}\left|x^{1} x^{1}\right| y\right)}{\Delta\left(x^{1}\left|x^{2}\right| y\right)} & \square_{x^{1} x^{2}}^{2}:=\frac{\Delta\left(x^{1}\left|x^{1} x^{2}\right| y\right)}{\Delta\left(x^{1}\left|x^{2}\right| y\right)} & \square_{x^{1} y}^{2}:=\frac{\Delta\left(x^{1}\left|x^{1} y\right| y\right)}{\Delta\left(x^{1}\left|x^{2}\right| y\right)} \\
\square_{x^{2} x^{2}}^{2}:=\frac{\Delta\left(x^{1}\left|x^{2} x^{2}\right| y\right)}{\Delta\left(x^{1}\left|x^{2}\right| y\right)} & \square_{x^{2} y}^{2}:=\frac{\Delta\left(x^{1}\left|x^{2} y\right| y\right)}{\Delta\left(x^{1}\left|x^{2}\right| y\right)} & \square_{y y}^{2}:=\frac{\Delta\left(x^{1}|y| y\right)}{\Delta\left(x^{1}\left|x^{2}\right| y\right)} \\
\square_{x^{1} x^{1}}^{3}:=\frac{\Delta\left(x^{1}\left|x^{2}\right| x^{1} x^{1}\right)}{\Delta\left(x^{1}\left|x^{2}\right| y\right)} & \square_{x^{1} x^{2}}^{3}:=\frac{\Delta\left(x^{1}\left|x^{2}\right| x^{1} x^{2}\right)}{\Delta\left(x^{1}\left|x^{2}\right| y\right)} & \square_{x^{1} y}^{3}:=\frac{\Delta\left(x^{1}\left|x^{2}\right| x^{1} y\right)}{\Delta\left(x^{1}\left|x^{2}\right| y\right)} \\
\square_{x^{2} x^{2}}^{3}:=\frac{\Delta\left(x^{1}\left|x^{2}\right| x^{2} x^{2}\right)}{\Delta\left(x^{1}\left|x^{2}\right| y\right)} & \square_{x^{2} y}^{3}:=\frac{\Delta\left(x^{1}\left|x^{2}\right| x^{2} y\right)}{\Delta\left(x^{1}\left|x^{2}\right| y\right)} & \square_{y y}^{3}:=\frac{\Delta\left(x^{1}\left|x^{2}\right| y y\right)}{\Delta\left(x^{1}\left|x^{2}\right| y\right)}
\end{array}\right.
$$

Thanks to these notations, we can rewrite the three equations (2.10), (2.22) and (2.23) in a more compact style. 
Lemma 2.29. A completely integrable system of three second order partial differential equations

$$
\left\{\begin{array}{l}
y_{x^{1} x^{1}}(x)=F^{1,1}\left(x^{1}, x^{2}, y(x), y_{x^{1}}(x), y_{x^{2}}(x)\right), \\
y_{x^{1} x^{2}}(x)=F^{1,2}\left(x^{1}, x^{2}, y(x), y_{x^{1}}(x), y_{x^{2}}(x)\right), \\
y_{x^{2} x^{2}}(x)=F^{2,2}\left(x^{1}, x^{2}, y(x), y_{x^{1}}(x), y_{x^{2}}(x)\right),
\end{array}\right.
$$

is equivalent to the simplest system $Y_{X^{1} X^{1}}=0, Y_{X^{1} X^{2}}=0, Y_{X^{2}, X^{2}}=0$, if and only if there exist local $\mathbb{K}$-analytic functions $X^{1}, X^{2}, Y$ such that it may be written under the specific form:

$$
\left\{\begin{aligned}
y_{x^{1} x^{1}}= & -\square_{x^{1} x^{1}}^{3}+y_{x^{1}} \cdot\left(-2 \square_{x^{1} y}^{3}+\square_{x^{1} x^{1}}^{1}\right)+y_{x^{2}} \cdot\left(\square_{x^{1} x^{1}}^{2}\right)+ \\
& +y_{x^{1}} y_{x^{1}} \cdot\left(-\square_{y y}^{3}+2 \square_{x^{1} y}^{1}\right)+y_{x^{1}} y_{x^{2}} \cdot\left(2 \square_{x^{1} y}^{2}\right)+ \\
& +y_{x^{1}} y_{x^{1}} y_{x^{1}} \cdot\left(\square_{y y}^{1}\right)+y_{x^{1}} y_{x^{1}} y_{x^{2}} \cdot\left(\square_{y y}^{2}\right), \\
y_{x^{1} x^{2}}= & -\square_{x^{1} x^{2}}^{3}+y_{x^{1}} \cdot\left(-\square_{x^{2} y}^{3}+\square_{x^{1} x^{2}}^{1}\right)+y_{x^{2}} \cdot\left(-\square_{x^{1} y}^{3}+\square_{x^{1} x^{2}}^{2}\right)+ \\
& +y_{x^{1}} y_{x^{1}} \cdot\left(\square_{x^{2} y}^{1}\right)+y_{x^{1}} y_{x^{2}} \cdot\left(-\square_{y y}^{3}+\square_{x^{1} y}^{1}+\square_{x^{2} y}^{2}\right)+ \\
& +y_{x^{2}} y_{x^{2}} \cdot\left(\square_{x^{1} y}^{2}\right)+y_{x^{1}} y_{x^{1}} y_{x^{2}} \cdot\left(\square_{y y}^{1}\right)+y_{x^{1}} y_{x^{2}} y_{x^{2}} \cdot\left(\square_{y y}^{2}\right), \\
y_{x^{2} x^{2}=}= & \square_{x^{2} x^{2}}^{3}+y_{x^{1}} \cdot\left(\square_{x^{2} x^{2}}^{1}\right)+y_{x^{2}} \cdot\left(-2 \square_{x^{2} y}^{3}+\square_{x^{2} x^{2}}^{2}\right)+ \\
& +y_{x^{1}} y_{x^{2}} \cdot\left(2 \square_{x^{2} y}^{1}\right)+y_{x^{2}} y_{x^{2}} \cdot\left(-\square_{y y}^{3}+2 \square_{x^{2} y}^{2}\right)+ \\
& +y_{x^{1}} y_{x^{2}} y_{x^{2}} \cdot\left(\square_{y y}^{1}\right)+y_{x^{2}} y_{x^{2}} y_{x^{2}} \cdot\left(\square_{y y}^{2}\right) .
\end{aligned}\right.
$$

2.32. General formulas. The formal dictionary between the original determinantial formulas (2.10), (2.22), (2.23), between the coefficients (2.15) of the second order prolongation of a vector field and between the new square formulas (2.31) above is evident. Consequently, without any computation, just by translating the family of formulas (2.14), we may deduce the exact formulation of the desired generalization of Lemma 2.29 above.

Lemma 2.33. A completely integrable system of second order partial differential equations of the form

$$
y_{x^{j_{1}} x^{j_{2}}}(x)=F^{j_{1}, j_{2}}\left(x, y(x), y_{x^{1}}(x), \ldots, y_{x^{n}}(x)\right), \quad j_{1}, j_{2}=1, \ldots n,
$$

is equivalent to the simplest system $Y_{X^{j_{1}} X^{j_{2}}}=0, j_{1}, j_{2}=1, \ldots, n$, if and only if there exist local $\mathbb{K}$-analytic functions $X^{l}, Y$ such that it may be written under the specific form:

$$
\left\{\begin{aligned}
y_{x^{j_{1}} x^{j_{2}}}= & -\square_{x^{j_{1}} x^{j_{2}}}^{n+1}+\sum_{k_{1}=1}^{n} y_{x^{k_{1}}} \cdot\left\{\left(\square_{x^{j_{1}} x^{j_{2}}}^{k_{1}}-\delta_{j_{1}}^{k_{1}} \square_{x^{j_{2}} y}^{n+1}-\delta_{j_{2}}^{k_{1}} \square_{x^{j_{1}}}^{n+1}\right)+\right. \\
& +y_{x^{j_{1}}} \cdot\left(\square_{x^{j_{2}} y}^{k_{1}}-\frac{1}{2} \delta_{j_{2}}^{k_{1}} \square_{y y}^{n+1}\right)+y_{x^{j_{2}}} \cdot\left(\square_{x^{j_{1}} y}^{k_{1}}-\frac{1}{2} \delta_{j_{1}}^{k_{1}} \square_{y y}^{n+1}\right)+ \\
& \left.+y_{x^{j_{1}}} y_{x^{j_{2}}} \cdot\left(\square_{y y}^{k_{1}}\right)\right\} .
\end{aligned}\right.
$$

Of course, to define the square functions in the context of $n \geqslant 2$ independent variables $\left(x^{1}, x^{2}, \ldots, x^{n}\right)$, we introduce the Jacobian determinant

$$
\Delta\left(x^{1}\left|x^{2}\right| \cdots\left|x^{n}\right| y\right):=\left|\begin{array}{cccc}
X_{x^{1}}^{1} & \cdots & X_{x^{n}}^{1} & X_{y}^{1} \\
\vdots & \cdots & \vdots & \vdots \\
X_{x^{1}}^{n} & \cdots & X_{x^{n}}^{n} & X_{y}^{n} \\
Y_{x^{1}} & \cdots & Y_{x^{n}} & Y_{y}
\end{array}\right|,
$$


together with its modifications

$$
\Delta\left(x^{1}|\cdots|^{k_{1}} x^{j_{1}} x^{j_{2}}|\cdots| y\right)
$$

in which the $k_{1}$-th column of partial first order derivatives $\left.\right|^{k_{1}} x^{k_{1}} \mid$ is replaced by the column $\left|{ }^{k_{1}} x^{j_{1}} x^{j_{2}}\right|$ of partial derivatives. Here, the indices $k_{1}, j_{1}, j_{2}$ satisfy $1 \leqslant k_{1}, j_{1}, j_{2} \leqslant$ $n+1$, with the convention that we adopt the notational equivalence

$$
x^{n+1} \equiv y \text {. }
$$

This convention will be convenient to write some of our general formulas in the sequel.

As we promised to only summarize the proof of Theorem 1.7 in this paper, we will not develope the proof of Lemma 2.33: it is similar to the proof of Lemma 3.32 in [Me2004].

\section{$\S 3$. FIRST AND SECOND AUXILIARY SYSTEM}

3.1. Functions $G_{j_{1}, j_{2}}, H_{j_{1}, j_{2}}^{k_{1}}, L_{j_{1}}^{k_{1}}$ and $M^{k_{1}}$. To discover the four families of functions appearing in the statement of Theorem 1.7, by comparing (2.35) and (1.10), it suffices (of course) to set:

$$
\left\{\begin{aligned}
G_{j_{1}, j_{2}} & :=-\square_{x^{j_{1}} x^{j_{2}}}^{n+1}, \\
H_{j_{1}, j_{2}}^{k_{1}} & :=\square_{x^{j_{1}} x^{j_{2}}}^{k_{1}}-\delta_{j_{1}}^{k_{1}} \square_{x^{j_{2}}}^{n+1}-\delta_{j_{2}}^{k_{1}} \square_{x^{j_{1}} y}^{n+1}, \\
L_{j_{1}}^{k_{1}} & :=2 \square_{x^{j_{1}} y}^{k_{1}}-\delta_{j_{1}}^{k_{1}} \square_{y y}^{n+1} \\
M^{k_{1}} & :=\square_{y y}^{k_{1}}
\end{aligned}\right.
$$

Consequently, we have shown the "only if" part of Theorem 1.7, which is the easiest implication.

To establish the "if" part, by far the most difficult implication, the very main lemma can be stated as follows.

Lemma 3.3. The partial differerential relations (I'), (II'), (III') and (IV') which express in length the compatibility conditions (1.11) are necessary and sufficient for the existence of functions $X^{l}, Y$ of $\left(x^{l_{1}}, y\right)$ satisfying the second order nonlinear system of partial differential equations (3.2) above.

Indeed, the collection of equations (3.2) is a system of partial differential equations with unknowns $X^{l}, Y$, by virtue of the definition of the square functions.

3.4. First auxiliary system. To proceed further, we observe that there are $(m+1)$ more square functions than functions $G_{j_{1}, j_{2}}, H_{j_{1}, j_{2}}^{k_{1}}, L_{j_{1}}^{k_{1}}$ and $M^{k_{1}}$. Indeed, a simple counting yields:

$$
\left\{\begin{array}{lr}
\#\left\{\square_{x^{j_{1}} x^{j_{2}}}^{k_{1}}\right\}=\frac{n^{2}(n+1)}{2}, & \#\left\{\square_{x^{j_{1}}}^{k_{1}}\right\}=n^{2}, \\
\#\left\{\square_{y y}^{k_{1}}\right\}=n, & \#\left\{\square_{x^{j_{1}} x^{j_{2}}}^{n+1}\right\}=\frac{n(n+1)}{2}, \\
\#\left\{\square_{x^{j_{1}}}^{n+1}\right\}=n, & \#\left\{\square_{y y}^{n+1}\right\}=1,
\end{array}\right.
$$

whereas

$$
\left\{\begin{array}{lr}
\#\left\{G^{j_{1}, j_{2}}\right\}=\frac{n(n+1)}{2}, & \#\left\{H_{j_{1}, j_{2}}^{k_{1}}\right\}=\frac{n^{2}(n+1)}{2}, \\
\#\left\{L_{j_{1}}^{k_{1}}\right\}=n^{2}, & \#\left\{M^{k_{1}}\right\}=n .
\end{array}\right.
$$

Here, the indices $j_{1}, j_{2}, k_{1}$ satisfy $1 \leqslant j_{1}, j_{2}, k_{1} \leqslant n$. Similarly as in [Me2004], to transform the system (3.2) in a true complete system, let us introduce functions $\Pi_{j_{1}, j_{2}}^{k_{1}}$ of 
$\left(x^{l_{1}}, y\right)$, where $1 \leqslant j_{1}, j_{2}, k_{1} \leqslant n+1$, which satisfy the symmetry $\Pi_{j_{1}, j_{2}}^{k_{1}}=\Pi_{j_{1}, j_{1}}^{k_{1}}$, and let us introduce the following first auxiliary system:

$$
\left\{\begin{array}{llrl}
\square_{x^{j_{1}} x^{j_{2}}}^{k_{1}}=\Pi_{j_{1}, j_{2}}^{k_{1}}, & \square_{x^{j_{1} y}}^{k_{1}}=\Pi_{j_{1}, n+1}^{k_{1}}, & \square_{y y}^{k_{1}}=\Pi_{n+1, n+1}^{k_{1}}, \\
\square_{x^{j_{1}} x^{j_{2}}}^{n+1}=\Pi_{j_{1}, j_{2}}^{n+1}, & \square_{x^{j_{1} y}}^{n+1}=\Pi_{j_{1}, n+1}^{n+1}, & \square_{y y}^{n+1}=\Pi_{n+1, n+1}^{n+1} .
\end{array}\right.
$$

It is complete. The necessary and sufficient conditions for the existence of solutions $X^{l}$, $Y$ follow by cross differentiations.

Lemma 3.8. For all $j_{1}, j_{2}, j_{3}, k_{1}=1,2, \ldots, n+1$, we have the cross differentiation relations

$$
\left(\square_{x^{j_{1}} x^{j_{2}}}^{k_{1}}\right)_{x^{j_{3}}}-\left(\square_{x^{j_{1}} x^{j_{3}}}^{k_{1}}\right)_{x^{j_{2}}}=-\sum_{k_{2}=1}^{n+1} \square_{x^{j_{1}} x^{j_{2}}}^{k_{2}} \square_{x^{j_{3}} x^{k_{2}}}^{k_{1}}+\sum_{k_{2}=1}^{n+1} \square_{x^{j_{1}} x^{j_{3}}}^{k_{2}} \square_{x^{j_{2}} x^{k_{2}}}^{k_{1}} .
$$

The proof of this lemma is exactly the same as the proof of Lemma 3.40 in [Me2004]. As a direct consequence, we deduce that a necessary and sufficient condition for the existence of solutions $\Pi_{j_{1}, j_{2}}^{k_{1}}$ to the first auxiliary system is that they satisfy the following compatibility partial differential relations:

$$
\frac{\partial \Pi_{j_{1}, j_{2}}^{k_{1}}}{\partial x^{j_{3}}}-\frac{\partial \Pi_{j_{1}, j_{3}}^{k_{1}}}{\partial x^{j_{2}}}=-\sum_{k_{2}=1}^{n=1} \Pi_{j_{1}, j_{2}}^{k_{2}} \cdot \Pi_{j_{3}, k_{2}}^{k_{1}}+\sum_{k_{2}=1}^{n=1} \Pi_{j_{1}, j_{3}}^{k_{2}} \cdot \Pi_{j_{2}, k_{2}}^{k_{1}},
$$

for all $j_{1}, j_{2}, j_{3}, k_{1}=1, \ldots, n+1$.

We shall have to specify this system in length according to the splitting $\{1,2, \ldots, n\}$ and $\{n+1\}$ of the indices of coordinates. We obtain six families of equations equivalent 
to (3.10) just above:

(3.11)

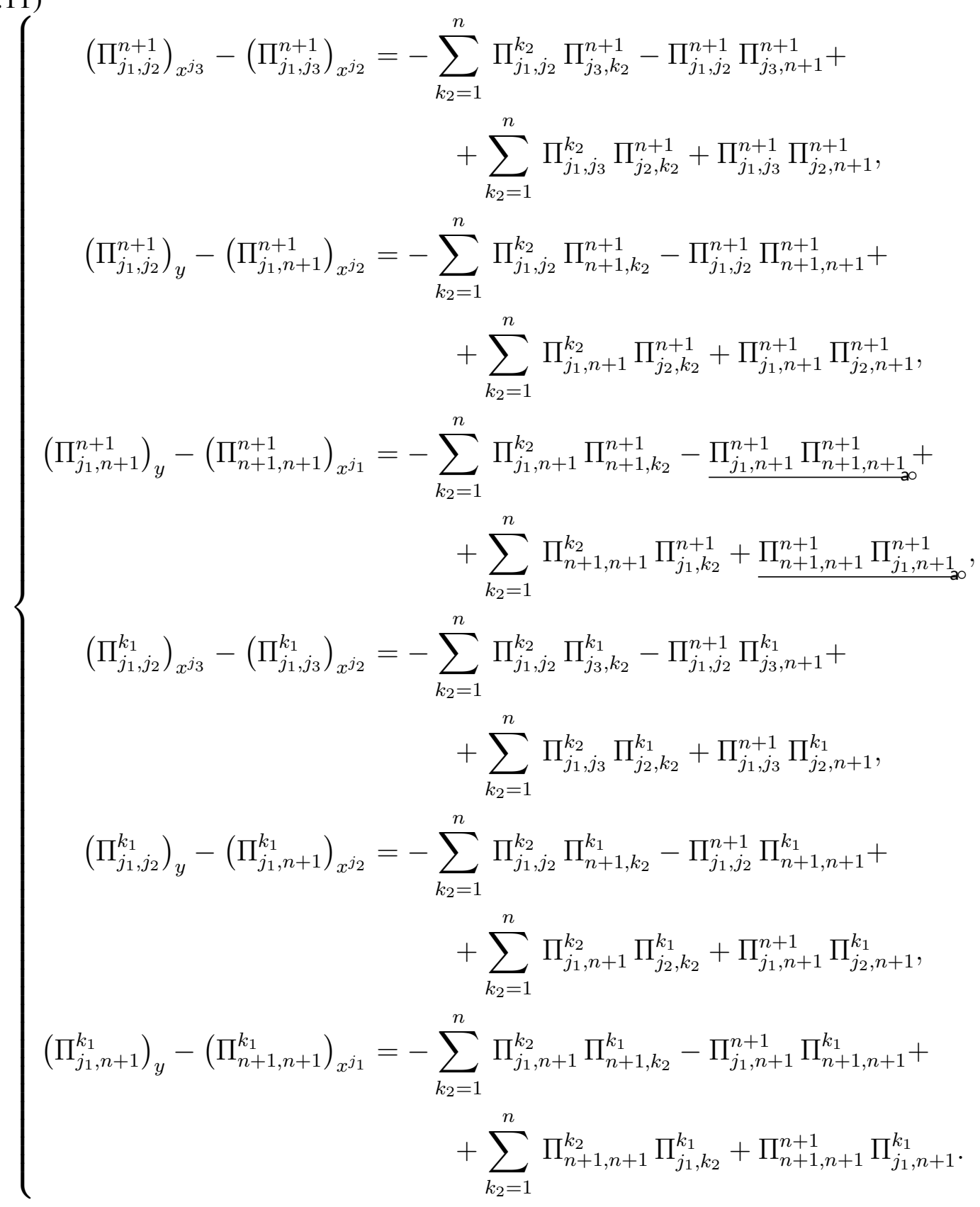

where the indices $j_{1}, j_{2}, j_{3}, k_{1}$ vary in the set $\{1,2,1, \ldots, n\}$.

3.12. Principal unknowns. As there are $(m+1)$ more square (or Pi) functions than the functions $G_{j_{1}, j_{2}}, H_{j_{1}, j_{2}}^{k_{1}}, L_{j_{1}}^{k_{1}}$ and $M^{k_{1}}$, we cannot invert directly the linear system (3.2). To quasi-inverse it, we choose the $(m+1)$ specific square functions

$$
\Theta^{1}:=\square_{x^{1} x^{1}}^{1}, \quad \Theta^{2}:=\square_{x^{2} x^{2}}^{2}, \cdots \cdots, \Theta^{n+1}:=\square_{x^{n+1} x^{n+1}}^{n+1},
$$


calling them principal unknowns, and we get the quasi-inversion:

$$
\left\{\begin{aligned}
\Pi_{j_{1}, j_{2}}^{k_{1}} & =\square_{x^{j_{1}} x^{j_{2}}}^{k_{1}}=H_{j_{1}, j_{2}}^{k_{1}}-\frac{1}{2} \delta_{j_{1}}^{k_{1}} H_{j_{2}, j_{2}}^{j_{2}}-\frac{1}{2} \delta_{j_{2}}^{k_{1}} H_{j_{1}, j_{1}}^{j_{1}}+\frac{1}{2} \delta_{j_{1}}^{k_{1}} \Theta^{j_{2}}+\frac{1}{2} \delta_{j_{2}}^{k_{1}} \Theta^{j_{1}} \\
\Pi_{j_{1}, n+1}^{k_{1}} & =\square_{x^{j_{1} y}}^{k_{1}}=\frac{1}{2} L_{j_{1}}^{k_{1}}+\frac{1}{2} \delta_{j_{1}}^{k_{1}} \Theta^{n+1} \\
\Pi_{n+1, n+1}^{k_{1}} & =\square_{y y}^{k_{1}}=M^{k_{1}} \\
\Pi_{j_{1}, j_{2}}^{n+1} & =\square_{x^{j_{1}} x^{j_{2}}}^{n+1}=-G_{j_{1}, j_{2}}, \\
\Pi_{j_{1}, n+1}^{n+1} & =\square_{x^{j_{1} y}}^{n+1}=-\frac{1}{2} H_{j_{1}, j_{1}}^{j_{1}}+\frac{1}{2} \Theta^{j_{1}} .
\end{aligned}\right.
$$

3.15. Second auxiliary system. Replacing the five families of functions $\Pi_{j_{1}, j_{2}}^{k_{1}}, \Pi_{j_{1}, n+1}^{k_{1}}$, $\Pi_{n+1, n+1}^{k_{1}}, \Pi_{j_{1}, j_{2}}^{n+1}, \Pi_{j_{1}, n+1}^{n+1}$ by their values obtained in (3.14) just above together with the principal unknowns

$$
\Pi_{j_{1}, j_{1}}^{j_{1}}=\Theta^{j_{1}}, \quad \Pi_{n+1, n+1}^{n+1}=\Theta^{n+1}
$$

in the six equations $(3.11)_{1},(3.11)_{2},(3.11)_{3},(3.11)_{4},(3.11)_{5}$ and $(3.11)_{6}$, after hard computations that we will not reproduce here, we obtain six families of equations. From now on, we abbreviate every sum $\sum_{k=1}^{n}$ as $\sum_{k_{1}}$.

Firstly:

$$
0=\underline{G_{j_{1}, j_{2}, x^{j_{3}}}-G_{j_{1}, j_{3}, x^{j_{2}}}}+\sum_{k_{1}} G_{j_{3}, k_{1}} H_{j_{1}, j_{2}}^{k_{1}}-\sum_{k_{1}} G_{j_{2}, k_{1}} H_{j_{1}, j_{3}}^{k_{1}}
$$

This is (I') of Theorem 1.7. Just above and below, we plainly underline the monomials involving a first order derivative. Secondly:

$$
\left\{\begin{aligned}
\Theta_{x^{j_{2}}}^{j_{1}}= & -2 G_{j_{1}, j_{2}, y}+H_{j_{1}, j_{1}, x^{j_{2}}}^{j_{1}}+ \\
& +\sum_{k_{1}} G_{j_{2}, k_{1}} L_{j_{1}}^{k_{1}}+\frac{1}{2} H_{j_{1}, j_{1}}^{j_{1}} H_{j_{2}, j_{2}}^{j_{2}}-\sum_{k_{1}} H_{j_{1}, j_{2}}^{k_{1}} H_{k_{1}, k_{1}}^{k_{1}}- \\
& -G_{j_{1}, j_{2}} \Theta^{n+1}-\frac{1}{2} H_{j_{1}, j_{1}}^{j_{1}} \Theta^{j_{2}}-\frac{1}{2} H_{j_{2}, j_{2}}^{j_{2}} \Theta^{j_{1}}+\sum_{k_{1}} H_{j_{1}, j_{2}}^{k_{1}} \Theta^{k_{1}}+ \\
& +\frac{1}{2} \Theta^{j_{1}} \Theta^{j_{2}}
\end{aligned}\right.
$$

Thirdly:

$$
\left\{\begin{aligned}
-\Theta_{x^{j_{1}}}^{n+1}+\frac{1}{2} \Theta_{y}^{j_{1}}= & \frac{1}{2} H_{j_{1}, j_{1}, y}^{j_{1}}- \\
& -\sum_{k_{1}} G_{j_{1}, k_{1}} M^{k_{1}}+\frac{1}{4} \sum_{k_{1}} H_{k_{1}, k_{1}}^{k_{1}} L_{j_{1}}^{k_{1}}+ \\
& +\frac{1}{4} H_{j_{1}, j_{1}}^{j_{1}} \Theta^{n+1}-\frac{1}{4} \sum_{k_{1}} L_{j_{1}}^{k_{1}} \Theta^{k_{1}}-\frac{1}{4} \Theta^{j_{1}} \Theta^{n+1}
\end{aligned}\right.
$$


Fourtly:

(3.20)

$$
\left\{\begin{aligned}
& \frac{1}{2} \delta_{j_{1}}^{k_{1}} \Theta_{x^{j_{3}}}^{j_{2}}-\frac{1}{2} \delta_{j_{1}}^{k_{1}} \Theta_{x^{j_{2}}}^{j_{3}}+\frac{1}{2} \delta_{j_{2}}^{k_{1}} \Theta_{x^{j_{3}}}^{j_{1}}-\frac{1}{2} \delta_{j_{3}}^{k_{1}} \Theta_{x^{j_{2}}}^{j_{1}}= \\
&=- \frac{H_{j_{1}, j_{2}, x^{j_{3}}}^{k_{1}}+H_{j_{1}, j_{3}, x^{j_{2}}}^{k_{1}}-\frac{1}{2} \delta_{j_{1}}^{k_{1}} H_{j_{3}, j_{3}, x^{j_{2}}}^{j_{3}}+\frac{1}{2} \delta_{j_{1}}^{k_{1}} H_{j_{2}, j_{2}, x^{j_{3}}}^{j_{2}}-}{\frac{1}{2} \delta_{j_{3}}^{k_{1}} H_{j_{1}, j_{1}, x^{j_{2}}}^{j_{1}}+\frac{1}{2} \delta_{j_{2}}^{k_{1}} H_{j_{1}, j_{1}, x^{j_{3}}}^{j_{1}}-} \\
&-\frac{1}{2} G_{j_{1}, j_{2}} L_{j_{3}}^{k_{1}}+\frac{1}{2} G_{j_{1}, j_{3}} L_{j_{2}}^{k_{1}}-\frac{1}{4} \delta_{j_{3}}^{k_{1}} H_{j_{1}, j_{1}}^{j_{1}} H_{j_{2}, j_{2}}^{j_{2}}+\frac{1}{4} \delta_{j_{2}}^{k_{1}} H_{j_{1}, j_{1}}^{j_{1}} H_{j_{3}, j_{3}}^{j_{3}}- \\
&-\sum \frac{1}{k_{2}} H_{j_{1}, j_{2}}^{k_{2}} H_{j_{3}, k_{2}}^{k_{1}}+\sum H_{k_{2}} H_{j_{1}, j_{3}}^{k_{2}} H_{j_{2}, k_{2}}^{k_{1}}-\frac{1}{2} \delta_{j_{2}}^{k_{1}} H_{j_{1}, j_{3}}^{k_{2}} H_{k_{2}, k_{2}}^{k_{2}}+\frac{1}{2} \delta_{j_{3}}^{k_{1}} H_{j_{1}, j_{2}}^{k_{2}} H_{k_{2}, k_{2}}^{k_{2}}- \\
&-\frac{1}{2} \delta_{j_{2}}^{k_{1}} G_{j_{1}, j_{3}} \Theta^{n+1}+\frac{1}{2} \delta_{j_{3}}^{k_{1}} G_{j_{1}, j_{2}} \Theta^{n+1}- \\
&-\frac{1}{4} \delta_{j_{2}}^{k_{1}} H_{j_{1}, j_{1}}^{j_{1}} \Theta^{j_{3}}+\frac{1}{4} \delta_{j_{3}}^{k_{1}} H_{j_{1}, j_{1}}^{j_{1}} \Theta^{j_{2}}-\frac{1}{4} \delta_{j_{2}}^{k_{1}} H_{j_{3}, j_{3}}^{j_{3}} \Theta^{j_{1}}+\frac{1}{4} \delta_{j_{3}}^{k_{1}} H_{j_{2}, j_{2}}^{j_{2}} \Theta^{j_{1}}- \\
&-\frac{1}{2} \delta_{j_{3}}^{k_{1}} \sum_{k_{1}} H_{j_{1}, j_{2}}^{k_{2}} \Theta^{k_{2}}+\frac{1}{2} \delta_{j_{2}}^{k_{1}} \sum_{k_{1}} H_{j_{1}, j_{3}}^{k_{2}} \Theta^{k_{2}}- \\
&-\frac{1}{4} \delta_{j_{3}}^{k_{1}} \Theta^{j_{1}} \Theta^{j_{2}}+\frac{1}{4} \delta_{j_{2}}^{k_{1}} \Theta^{j_{1}} \Theta^{j_{3}} \cdot
\end{aligned}\right.
$$

Fifthly:

(3.21)

$$
\left\{\begin{aligned}
\frac{1}{2} \delta_{j_{1}}^{k_{1}} \Theta_{y}^{j_{2}}+\frac{1}{2} \delta_{j_{2}}^{k_{1}} \Theta_{y}^{j_{1}}-\frac{1}{2} \delta_{j_{1}}^{k_{1}} \Theta_{x^{j_{2}}}^{n+1}= & \\
= & G_{j_{1}, j_{2}} M^{k_{1}}+\frac{1}{2} \sum_{k_{2}} H_{j_{1}, k_{2}}^{k_{1}} L_{j_{1}}^{k_{2}}-\frac{1}{2} \sum_{k_{2}} H_{j_{1}, j_{2}}^{k_{2}} L_{k_{2}}^{k_{1}}-\frac{1}{4} \delta_{j_{2}}^{k_{1}} \sum_{k_{2}} H_{k_{2}, k_{2}}^{k_{2}} L_{j_{1}}^{k_{2}}- \\
& -\frac{1}{4} \delta_{j_{2}}^{k_{1}} H_{j_{1}, j_{1}}^{j_{1}} \Theta^{n+1}+\frac{1}{4} \delta_{j_{2}}^{k_{1}} \sum_{k_{2}} L_{j_{1}}^{k_{2}} \Theta^{k_{2}}+\frac{1}{4} \delta_{j_{2}}^{k_{1}} \Theta^{j_{1}} \Theta^{n+1}
\end{aligned}\right.
$$

Sixthly:

$$
\left\{\begin{aligned}
\delta_{j_{1}}^{k_{1}} \Theta_{y}^{n+1}= & -\frac{L_{j_{1}, y}^{k_{1}}+2 M_{x^{j_{1}}}^{k_{1}}}{}\{ \\
& +2 \sum_{k_{2}} H_{j_{1}, k_{2}}^{k_{1}} M^{k_{2}}-\delta_{j_{1}}^{k_{1}} \sum_{k_{2}} H_{k_{2}, k_{2}}^{k_{2}} M^{k_{2}}-\frac{1}{2} \sum_{k_{2}} L_{j_{1}}^{k_{2}} L_{k_{2}}^{k_{1}}+ \\
& +\delta_{j_{1}}^{k_{1}} \sum_{k_{2}} M^{k_{2}} \Theta^{k_{2}}+\frac{1}{2} \delta_{j_{1}}^{k_{1}} \Theta^{n+1} \Theta^{n+1}
\end{aligned}\right.
$$

3.23. Solving $\Theta_{x^{j_{2}}}^{j_{1}}, \Theta_{y}^{j_{1}}, \Theta_{x^{j_{1}}}^{n+1}$ and $\Theta_{y}^{n+1}$. From the six families of equations (3.17), (3.18), (3.19), (3.20), (3.21) and (3.22), we can solve $\Theta_{x^{j_{2}}}^{j_{1}}, \Theta_{y}^{j_{1}}, \Theta_{x^{j_{1}}}^{n+1}$ and $\Theta_{y}^{n+1}$. Not mentioning the (hard) intermediate computations, we obtain firstly:

$$
\left\{\begin{aligned}
\Theta_{x^{j_{2}}}^{j_{1}}= & -2 G_{j_{1}, j_{2}, y}+H_{j_{1}, j_{1}, x^{j_{2}}}^{j_{1}} \\
& +\sum_{l} G_{j_{2}, l} L_{j_{1}}^{l}+\frac{1}{2} H_{j_{1}, j_{1}}^{j_{1}} H_{j_{2}, j_{2}}^{j_{2}}-\sum_{l} H_{j_{1}, j_{2}}^{l} H_{l, l}^{l}- \\
& -G_{j_{1}, j_{2}} \Theta^{n+1}-\frac{1}{2} H_{j_{1}, j_{1}} \Theta^{j_{1}}-\frac{1}{2} H_{j_{2}, j_{2}}^{j_{2}} \Theta^{j_{1}}+\sum_{l} H_{j_{1}, j_{2}}^{l} \Theta^{l}+\frac{1}{2} \Theta^{j_{1}} \Theta^{j_{2}}
\end{aligned}\right.
$$


Secondly:

(3.25)

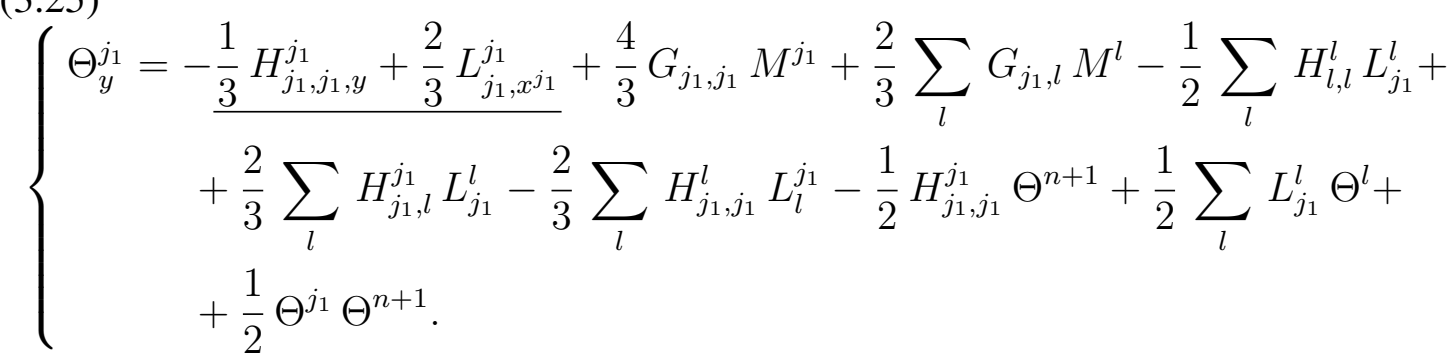

Thirdly:

(3.26)

$$
\left\{\begin{aligned}
\Theta_{x^{j_{1}}}^{n+1}=- & \frac{2}{3} H_{j_{1}, j_{1}, y}^{j_{1}}+\frac{1}{3} L_{j_{1}, x^{j_{1}}}^{j_{1}} \\
& +\frac{2}{3} G_{j_{1}, j_{1}} M^{j_{1}}+\frac{4}{3} \sum_{l} G_{j_{1}, l} M^{l}-\frac{1}{2} \sum_{l} H_{l, l}^{l} L_{j_{1}}^{l}+ \\
& +\frac{1}{3} \sum_{l} H_{j_{1}, l}^{j_{1}} L_{j_{1}}^{l}-\frac{1}{3} \sum_{l} H_{j_{1}, j_{1}}^{l} L_{l}^{j_{1}}-\frac{1}{2} H_{j_{1}, j_{1}}^{j_{1}} \Theta^{n+1}+\frac{1}{2} \sum_{l} L_{j_{1}}^{l} \Theta^{l}+ \\
& +\frac{1}{2} \Theta^{j_{1}} \Theta^{n+1} .
\end{aligned}\right.
$$

Fourtly:

$$
\left\{\begin{aligned}
\Theta_{y}^{n+1}= & -\frac{L_{j_{1}, y}^{j_{1}}+2 M_{x^{j_{1}}}^{j_{1}}}{}+2 \sum_{l} H_{j_{1}, l}^{j_{1}} M^{l}-\sum_{l} H_{l, l}^{l} M^{l}-\frac{1}{2} \sum_{l} L_{j_{1}}^{l} L_{l}^{j_{1}}+ \\
& +\sum_{l} M^{l} \Theta^{l}+\frac{1}{2} \Theta^{n+1} \Theta^{n+1} .
\end{aligned}\right.
$$

These four families of partial differential equations constitute the second auxiliary system. By replacing these solutions in the three remaining families of equations (3.20), (3.21) and (3.22), we obtain supplementary equations (which we do not copy) that are direct consequences of (I'), (II'), (III'), (IV').

To complete the proof of the main Lemma 3.3 above, it suffices now to establish the first implication of the following list, since the other three have been already established.

- Some given functions $G_{j_{1}, j_{2}}, H_{j_{1}, j_{2}}^{k_{1}}, L_{j_{1}}^{k_{1}}$ and $M^{k_{1}}$ of $\left(x^{l_{1}}, y\right)$ satisfy the four families of partial differential equations (I'), (II'), (III') and (IV') of Theorem 1.7. $\Downarrow$

- There exist functions $\Theta^{j_{1}}, \Theta^{n+1}$ satisfying the second auxiliary system (3.24), (3.25), (3.26) and (3.27).

$\Downarrow$

- These solution functions $\Theta^{j_{1}}, \Theta^{n+1}$ satisfy the six families of partial differential equations (3.17), (3.18), (3.19), (3.20), (3.21) and (3.22).

$\Downarrow$

- There exist functions $\Pi_{j_{1}, j_{2}}^{k_{1}}$ of $\left(x^{l_{1}}, y\right), 1 \leqslant j_{1}, j_{2}, k_{1} \leqslant m+1$, satisfying the first auxiliary system (3.7) of partial differential equations.

$\Downarrow$

- There exist functions $X^{l_{2}}, Y$ of $\left(x^{l_{1}}, y\right)$ transforming the system $y_{x^{j_{1}} x^{j_{2}}}=$ $F^{j_{1}, j_{2}}\left(x^{l_{1}}, y, y_{x_{2}}\right), j_{1}, j_{2}=1, \ldots, n$, to the simplest system $Y_{X^{j_{1}} X^{j_{2}}}=0$, $j_{1}, j_{1}=1, \ldots, n$.

3.28. Compatibility conditions for the second auxiliary system. We notice that the second auxiliary system is also a complete system. Thus, to establish the first above 
implication, it suffices to show that the four families of compatibility conditions:

$$
\left\{\begin{array}{l}
0=\left(\Theta_{x^{j_{2}}}^{j_{1}}\right)_{x^{j_{3}}}-\left(\Theta_{x^{j_{3}}}^{j_{1}}\right)_{x^{j_{2}}}, \\
0=\left(\Theta_{x^{j_{2}}}^{j_{1}}\right)_{y}-\left(\Theta_{y}^{j_{1}}\right)_{x^{j_{2}}}, \\
0=\left(\Theta_{x^{j_{1}}}^{n+1}\right)_{x^{j_{2}}}-\left(\Theta_{x^{j_{2}}}^{n+1}\right)_{x^{j_{1}}} \\
0=\left(\Theta_{x^{j_{2}}}^{n+1}\right)_{y}-\left(\Theta_{y}^{n+1}\right)_{x^{j_{2}}},
\end{array}\right.
$$

are a consequence of (I'), (I'), (III'), (IV').

For instance, in $(3.29)_{1}$, replacing $\Theta_{x^{j_{2}}}^{j_{1}}$ by its expression (3.24), differentiating it with respect to $x^{j_{3}}$, replacing $\Theta_{x^{j_{3}}}^{j_{1}}$ by its expression (3.24), differentiating it with respect to $x^{j_{2}}$ and substracting, we get:

(3.30)

$$
\begin{aligned}
& \left(0=-2 G_{j_{1}, j_{2}, y x^{j_{3}}}+2 G_{j_{1}, j_{3}, y x^{j_{2}}}+\underline{H_{j_{1}, j_{1}, x^{j_{2}} x^{j_{3}}}^{j_{1}}} \mathrm{a}-\underline{H_{j_{1}, j_{1}, x^{j_{3} x^{j_{2}}}}^{j_{1}} \mathrm{a}}+\right. \\
& +\frac{1}{2} \Theta_{x^{j_{3}}}^{j_{1}} \Theta^{j_{2}}+\frac{1}{2} \Theta^{j_{1}} \underline{\Theta_{x^{j_{3}}}^{j_{2}}}-\frac{1}{2} \Theta_{x^{j_{2}}}^{j_{1}} \Theta^{j_{3}}-\frac{1}{2} \Theta^{j_{1}} \underline{\Theta_{x^{j_{2}}}^{j_{3}}}- \\
& -\frac{1}{2} H_{j_{1}, j_{1}, x^{j_{3}}}^{j_{1}} \Theta^{j_{2}}-\frac{1}{2} H_{j_{1}, j_{1}}^{j_{1}} \underline{\Theta_{x^{j_{3}}}^{j_{2}}}+\frac{1}{2} H_{j_{1}, j_{1}, x^{j_{2}}}^{j_{1}} \Theta^{j_{3}}+\frac{1}{2} H_{j_{1}, j_{1}}^{j_{1}} \underline{\Theta_{x^{j_{2}}}^{j_{3}}}- \\
& -\frac{1}{2} H_{j_{2}, j_{2}, x^{j_{3}}}^{j_{2}} \Theta^{j_{1}}-\frac{1}{2} H_{j_{2}, j_{2}}^{j_{2}} \underline{\Theta_{x^{j_{3}}}^{j_{1}}}+\frac{1}{2} H_{j_{3}, j_{3}, x^{j_{2}}}^{j_{3}} \Theta^{j_{1}}+\frac{1}{2} H_{j_{3}, j_{3}}^{j_{3}} \underline{\Theta_{x^{j_{2}}}^{j_{1}}}- \\
& -G_{j_{1}, j_{2}, x^{j_{3}}} \Theta^{n+1}-G_{j_{1}, j_{2}} \underline{\Theta_{x^{j_{3}}}^{n+1}}+G_{j_{1}, j_{3}, x^{j_{2}}} \Theta^{n+1}+G_{j_{1}, j_{3}} \underline{\Theta_{x^{j_{2}}}^{n+1}}+ \\
& +\sum_{l} H_{j_{1}, j_{2}, x^{j_{3}}}^{l} \Theta^{l}+\sum_{l} H_{j_{1}, j_{2}}^{l} \underline{\Theta_{x^{j_{3}}}^{l}}-\sum_{l} H_{j_{1}, j_{3}, x^{j_{2}}}^{l} \Theta^{l}-\sum_{l} H_{j_{1}, j_{3}}^{l} \underline{\Theta_{x^{j_{2}}}^{l}}+ \\
& +\frac{1}{2} H_{j_{1}, j_{1}, x^{j_{3}}}^{j_{1}} H_{j_{2}, j_{2}}^{j_{2}}+\frac{1}{2} H_{j_{1}, j_{1}}^{j_{1}} H_{j_{2}, j_{2}, x^{j_{3}}}^{j_{2}}-\frac{1}{2} H_{j_{1}, j_{1}, x^{j}}^{j_{1}} H_{j_{3}, j_{3}}^{j_{3}}-\frac{1}{2} H_{j_{1}, j_{1}}^{j_{1}} H_{j_{3}, j_{3}, x^{j_{2}}}^{j_{3}}- \\
& -\sum_{l} H_{j_{1}, j_{2}, x^{j_{3}}}^{l} H_{l, l}^{l}-\sum_{l} H_{j_{1}, j_{2}}^{l} H_{l, l, x^{j_{3}}}^{l}+\sum_{l} H_{j_{1}, j_{3}, x^{j_{2}}}^{l} H_{l, l}^{l}+\sum_{l} H_{j_{1}, j_{3}}^{l} H_{l, l, x^{j_{2}}}^{l}+ \\
& +\sum_{l} G_{j_{2}, l, x^{j_{3}}} L_{j_{1}}^{l}+\sum_{l} G_{j_{2}, l} L_{j_{1}, x^{j_{3}}}^{l}-\sum_{l} G_{j_{3}, l, x^{j_{2}}} L_{j_{1}}^{l}-\sum_{l} G_{j_{3}, l} L_{j_{1}, x^{j_{2}}}^{l} .
\end{aligned}
$$

Next, replacing the twelve first order partial derivatives underlined just above:

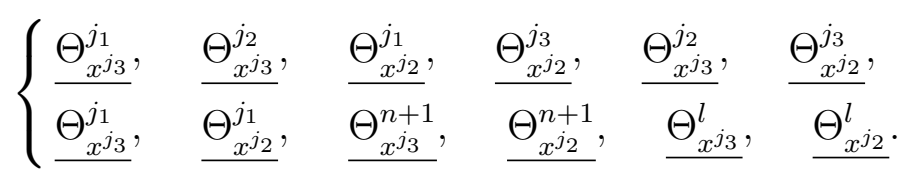


by their values issued from (3.24), (3.26) and adapting the summation indices, we get the explicit developed form of the first family of compatibility conditions $(3.29)_{1}$ :

(3.32)

$$
\begin{aligned}
& \begin{aligned}
0=?=- & \frac{2 G_{j_{1}, j_{2}, x^{j_{3} y}}+2 G_{j_{1}, j_{3}, x^{j_{2} y}}}{} \\
& -\sum_{l} G_{j_{3}, l, x^{j_{2}}} L_{j_{1}}^{l}+\sum_{l} G_{j_{2}, l, x^{j_{3}}} L_{j_{1}}^{l}-G_{j_{1}, j_{2}, y} H_{j_{3}, j_{3}}^{j_{3}}+G_{j_{1}, j_{3}, y} H_{j_{2}, j_{2}}^{j_{2}}-
\end{aligned} \\
& -2 \sum_{l} G_{l, j_{3}} H_{j_{1}, j_{2}}^{l}+2 \sum_{l} G_{l, j_{2}} H_{j_{1}, j_{3}}^{l}-\sum_{l} H_{j_{1}, j_{2}, x^{j} 3}^{l} H_{l, l}^{l}+\sum_{l} H_{j_{1}, j_{3}, x^{j_{2}}}^{l} H_{l, l}^{l}- \\
& -\underline{\frac{2}{3} H_{j_{2}, j_{2}, y}^{j_{2}} G_{j_{1}, j_{3}}+\frac{2}{3} H_{j_{3}, j_{3}, y}^{j_{3}} G_{j_{1}, j_{2}}-\frac{2}{3} L_{j_{3}, x^{j_{3}}}^{j_{3}} G_{j_{1}, j_{2}}+\frac{2}{3} L_{j_{2}, x^{j_{2}}}^{j_{2}} G_{j_{1}, j_{3}}-} \\
& -\sum_{l} L_{j_{1}, x^{j_{2}}}^{l} G_{j_{3}, l}+\sum_{l} L_{j_{1}, x^{j_{3}}}^{l} G_{j_{2}, l}- \\
& -\overline{\frac{2}{3} G_{j_{1}, j_{2}} G_{j_{3}, j_{3}} M^{j_{3}}+\frac{2}{3} G_{j_{1}, j_{3}} G_{j_{2}, j_{2}}} M^{j_{2}}-\frac{4}{3} \sum_{l} G_{j_{1}, j_{2}} G_{j_{3}, l} M^{l}+ \\
& +\frac{4}{3} \sum_{l} G_{j_{1}, j_{3}} G_{j_{2}, l} M^{l}-\frac{1}{2} \sum_{l} G_{j_{3}, l} H_{j_{1}, j_{1}}^{j_{1}} L_{j_{2}}^{l}+\frac{1}{2} \sum_{l} G_{j_{2}, l} H_{j_{1}, j_{1}}^{j_{1}} L_{j_{3}}^{l}- \\
& -\frac{1}{2} \sum_{l} G_{j_{3}, l} H_{j_{2}, j_{2}}^{j_{2}} L_{j_{1}}^{l}+\frac{1}{2} \sum_{l} G_{j_{2}, l} H_{j_{3}, j_{3}}^{j_{3}} L_{j_{1}}^{l}-\frac{1}{2} \sum_{l} G_{j_{1}, j_{3}} H_{l, l}^{l} L_{j_{2}}^{l}+ \\
& +\frac{1}{2} \sum_{l} G_{j_{1}, j_{2}} H_{l, l}^{l} L_{j_{3}}^{l}-\frac{1}{3} \sum_{l} G_{j_{1}, j_{2}} H_{j_{3}, l}^{j_{3}} L_{j_{3}}^{l}+\frac{1}{3} \sum_{l} G_{j_{1}, j_{3}} H_{j_{2}, l}^{j_{2}} L_{j_{2}}^{l}- \\
& -\frac{1}{3} G_{j_{1}, j_{3}} H_{j_{2}, j_{2}}^{l} L_{l}^{j_{2}}+\frac{1}{3} G_{j_{1}, j_{2}} H_{j_{3}, j_{3}}^{l} L_{l}^{j_{3}}- \\
& -\sum_{l} \sum_{p} G_{j_{2}, p} H_{j_{1}, j_{3}}^{l} L_{l}^{p}+\sum_{l} \sum_{p} G_{j_{3}, p} H_{j_{1}, j_{2}}^{l} L_{l}^{p}- \\
& -\sum_{l} \sum_{p} H_{j_{1}, j_{2}}^{l} H_{l, j_{3}}^{p} H_{p, p}^{p}+\sum_{l} \sum_{p} H_{j_{1}, j_{3}}^{l} H_{l, j_{2}}^{p} H_{p, p}^{p} .
\end{aligned}
$$

Lemma 3.33. ([Me2003, Me2004]) This first family of compatibility conditions for the second auxiliary system obtained by developing $(3.29)_{1}$ in length, together with the three remaining families obtained by developing $(3.29)_{2},(3.29)_{3},(3.29)_{4}$ in length, are consequences, by linear combinations and by differentiations, of (I'), (II'), (III'), (IV'), of Theorem 1.7.

The summarized proof of Theorem 1.7 is complete. 


\section{IV: Bibliography}

\section{REFERENCES}

[Ar1988] ARNOL'D, V.I.: Dynamical systems. I. Ordinary differential equations and smooth dynamical systems, Translated from the Russian. Edited by D. V. Anosov and V. I. Arnol'd. Encyclopaedia of Mathematical Sciences, vol. 1. Springer-Verlag, Berlin, 1988. x+233 pp.

[Ar1968] ARTIN, M.: On the solutions of analytic equations, Invent. Math. 5 (1968), $277-291$.

[BER1999] BAOUENDI, M.S.; EBENFELT, P.; RothSCHILD, L.P.: Real submanifolds in complex space and their mappings. Princeton Mathematical Series, vol. 47, Princeton University Press, Princeton, NJ, 1999, xii+404 pp.

[BJT1985] BaOUend, M.S.; JaCOBOwITZ, H.; Treves, F.: On the analyticity of CR mappings, Ann. of Math. 122 (1985), no. 2, 365-400.

[Bel1996] Bellaïche, A.: SubRiemannian Geometry, Progress in Mathematics, vol. 144, Birkhäuser Verlag, Basel/Switzerland, 1996, 1-78.

[Be1979] BELOSHAPKA, V.K.: On the dimension of the group of automorphisms of an analytic hypersurface, Izv. Akad. Nauk SSSR Ser. Mat. 43 (1979), 243-266; English transl. in Math. USSR-Izv. 14 (1980), 223-245.

[Be1988] BeLOSHAPKA, V.K.: Finite-dimensionality of the group of automorphisms of a real-analytic surface, Izv. Akad. Nauk SSSR Ser. Mat. 52 (1988), 437-442; English transl. in Math. USSRIzv. 32 (1989), 443-448.

[Be1997] BelOSHAPKA, V.K.: CR-varieties of the type $(1,2)$ as varieties of 'super-high' codimension, Russian J. Math. Phys. 5 (1997), 399-404.

[Be2002] BeloshAPKA, V.K.: Real submanifolds in complex space: polynomial models, automorphisms, and classification problems, Uspekhi Mat. Nauk 57 (2002), no. 1, 3-44; English transl. in Russian Math. Surveys 57 (2002), no. 1, 1-41.

[BES2005] BeloshapKa, V.K.; EZHov, V.; Schmalz, G.: Canonical Cartan connection and holomorphic invariants of Engel CR manifolds, arxiv.org/abs/math. CV/0508084.

[BK1989] Bluman, G.W.; KumeI, S.: Symmetries and differential equations, Springer Verlag, Berlin, 1989.

[Bo1991] Boggess, A.: CR manifolds and the tangential Cauchy-Riemann complex. Studies in Advanced Mathematics. CRC Press, Boca Raton, FL, 1991, xviii+364 pp.

[Bo1972] BourbaKI, N.: Groupes at algèbres de Lie, chapitre 2, Hermann, Paris, 1972.

[BSW1978] BURNS, D.Jr.; SHNIDER, S.; WELLS, R.O.Jr.: Deformations of strictly pseudoconvex domains Invent. Math. 46 (1978), no. 3, 237-253.

[Ca1922] CARTAN, É.: Sur les équations de la gravitation d'Einstein, J. Math. pures et appl. 1 (1922), 141-203.

[Ca1924] CARTAN, É.: Sur les variétés à connexion projective, Bull. Soc. Math. France 52 (1924), 205241.

[Ca1932a] CARTAN, É.: Sur la géométrie pseudo-conforme des hypersurfaces de l'espace de deux variables complexes, I, Ann. Math. Pura Appl. 11 (1932), 17-90.

[Ca1932b] CARTAN, É.: Sur la géométrie pseudo-conforme des hypersurfaces de l'espace de deux variables complexes, II, Ann. Scuola Norm. Sup. Pisa 1 (1932), 333-354.

[Ca1937] CARTAN, É.: Les problèmes d'équivalence (Séminaire de Math., 4 année, 1936-37), Euvres complètes, II, 1311-1334, Gauthier-Villars, Paris, 1953.

[CM1974] CHERN, S.S.; Moser, J.K.: Real hypersurfaces in complex manifolds, Acta Math. 133 (1974), no. 2, 219-271.

[Ch1975] CHERN, S.-S.: On the projective structure of a real hypersurface in $\mathbb{C}^{n+1}$, Math. Scand. 36 (1975), 74-82.

[Ch1989] ChIRKA, E.M.: Complex analytic sets, Mathematics and its applications (Soviet Series), vol. 46. Kluwer Academic Publishers Group, Dordrecht, 1989. xx+372 pp.

[Ch1991] CHIRKA, E.M.: An introduction to the geometry of CR manifolds (Russian), Uspekhi Mat. Nauk 46 (1991), no. 1(277), 81-164, 240; translation in Russian Math. Surveys 46 (1991), no. $1,95-197$

[CS1996] Constantine, G.M.; SAvits, T.H.: A multivariate Fà̀ di Bruno formula with applications, Trans. Amer. Math. Soc. 348 (1996), no. 2, 503-520.

[DF1988] DIEDERICH, K.; FORNÆSS, J.E.: Proper holomorphic mappings between real-analytic pseudoconvex domains in $\mathbb{C}^{n}$, Math. Ann 282 (1988), no. 4, 681-700. 
[DP2003] DIEDERICH, K.; PINCHUK, S.: Regularity of continuous CR-maps in arbitrary dimension, Michigan Math. J. 51 (2003), 111-140. Erratum: ib., no. 3, 667-668.

[DW1980] DIEDERICH, K.; WEBSTER, S.M.: A reflection principle for degenerate real hypersurfaces, Duke Math. J. 47 (1980), no. 4, 835-843.

[Eb1998] EBEnfElt, P.: Normal forms and biholomorphic equivalences of real hypersurfaces in $\mathbb{C}^{3}$, Indiana Univ. Math. J. 47 (1998), 311-366.

[Eb2006] Ebenfelt, P.: Correction to "Uniformly Levi degenerate CR manifolds: the 5-dimensional case, Duke Math. J. 131 (2006), 589-591.

[EL1890] ENGEL, F.; LIE, S.: Theorie der Transformationsgruppen, I, II, II, Teubner, Leipzig, 1889, $1891,1893$.

[EKV1985] Ezhov, V.V.; Kruzhilin, N.G.; Vitushkin, A.G.: Continuation of holomorphic mappings along real-analytic hypersurfaces (Russian). Current problems in mathematics. Mathematical analysis, algebra, topology. Trudy Mat. Inst. Steklov 167 (1985), 60-95, 276.

[Fa1980] FARAN, J.: Segre families and real hypersurfaces, Invent. Math. 60 (1980), no. 2, 135-172.

[F1969] FEDERER, H.: Geometric measure theory, Die Grundlehren der Mathematischen Wissenschaften, Band 153, Springer Verlag, New York, 1969, xiv+676 pp. Springer-Verlag, Berlin, 1969.

[Fe1995] FELS, M.: The equivalence problem for systems of second-order ordinary differential equations, Proc. London Math. Soc. 71 (1995), no. 2, 221-240.

[FK2005a] FELS, G.; Kaup, W.: CR-manifolds of dimension 5: A Lie algebra approach, J. Reine Angew. Math., to appear. arxiv.org/abs/math.DG/050811.

[FK2005b] FELS, G.; Kaup, W.: Homogeneous Levi degenerate CR-manifolds in dimension 5, to appear.

[Fr1877] Frobenius, G.: Ueber das Pfaffsche Problem, J. Reine Angew. Math. 82 (1877), 230-315.

[G1989] GARDNER, R.B.: The method of equivalence and its applications, CBMS-NSF Regional Conference Series in Applied Mathematics, vol. 58 (SIAM, Philadelphia, 1989), 127 pp.

[GM2003a] GAUSSIER, H.; MERKER, J.: Symmetries of partial differential equations, J. Korean Math. Soc. 40 (2003), no. 3, 517-561; e-print: http://fr.arxiv.org/abs/math. CV/0404127.

[GM2003b] GAUSSIER, H.; MERKER, J.: A new example of uniformly Levi nondegenerate hypersurface in $\mathbb{C}^{3}$, Ark. Mat. 41 (2003), no. 1, 85-94.

[GM2004] GAUSSIER, H.; MERKER, J.: Nonalgebraizable real analytic tubes in $\mathbb{C}^{n}$, Math. Z. 247 (2004), no. 2, 337-383.

[GM2006] GAUSSIER, H.; MERKer, J.: Erratum to "A new example of a uniformly Levi degenerate hypersurface in $\mathbb{C}^{3}$ ”, 2006, to appear.

[GV1987] Gershrovich, V.Ya.; Vershik, A.M.: Nonholonomic dynamical systems. Geometry of distributions and variational problems. Dynamical Systems VII, Encyclopædia of mathematical sciences, vol. 16, V.I. Arnol'd and S.P. Novikov (Eds.), 1-81, Springer-Verlag, Berlin, 1994.

[Gr2005] DE GRAAF, W.A.: Classification of solvable Lie algebras, Experiment. Math. 14 (2005), no. 1, $15-25$.

[GTW1989] GRISSOM, C.; ThOMPSOn, G.; Wilkens, G.: Linearization of second order ordinary differential equations via Cartan's equivalence method, J. Diff. Eq. 77 (1989), no. 1, 1-15.

[Gr2000] GRossmAN, D.A.: Torsion-free path geometries and integrable second order ODE systems, Selecta Math. (N.S.) 6 (2000), no. 4, 399-442.

[Ha1937] Hachtroudi, M.: Les espaces d'éléments à connexion projective normale, Actualités Scientifiques et Industrielles, vol. 565, Paris, Hermann, 1937.

[Ha1982] HaRTMan, P.: Ordinary Differential Equations. Birkhäuser, Boston 1982.

[Ha2003] HAUSER, H.: The Hironaka theorem on resolution of singularities (or: A proof we always wanted to understand), Bull. Amer. Math. Soc. (N.S.) 40 (2003), no. 3, 323-403.

[Hi1976] HiRsCH, M.W.: Differential topology, Graduate Texts in Mathematics, 33, Springer-Verlag, Berlin, 1976, x+222 pp.

[HK1989] HSU, L.; KAMRAN, N.: Classification of second order ordinary differential equations admitting Lie groups of fibre-preserving point symmetries, Proc. London Math. Soc. 58 (1989), no. 3, 387-416.

[Ib1992] IBRAGIMOV, N.H.: Group analysis of ordinary differential equations and the invariance principle in mathematical physics, Russian Math. Surveys 47:4 (1992), 89-156.

[Ib1999] IBRAGIMOV, N.H.: Elementary Lie group analysis and ordinary differential equations, Wiley Series in Mathematical Methods in Practice, 4. John Wiley \& Sons, Ltd., Chichester, 1999. xviii+347 pp. 
[IL2003] IVEY, J.A.; LANDSBERG, J.M.: Cartan for beginners: differential geometry via moving frames and exterior differential systems, Graduate Studies in Mathematics, 61. American Mathematical Society, Providence, RI, 2003. xiv+378 pp.

[Ja1990] JaCOBOWITZ, An introduction to CR structures, Math. Surveys and Monographs, 32. Amer. Math. Soc., Providence, 1990. x+237 pp.

[Ji2002] JI, S.: Algebraicity of real analytic hypersurfaces with maximal rank, Amer. J. Math. 124 (2002), no. 6, 1083-1102.

[JoPf2000] DE Jong, T.; Pfister, G.: Local analytic geometry. Basic theory and applications, Advanced Lectures in Mathematics. Friedr. Vieweg \& Sohn, Braunschweig, 2000. xii+382 pp.

[Kn2004] KNAPP, A.W.: Lie groups beyond an introduction, Progress in Mathematics, 140, Birkhäuser, Basel, third edition, 2004, xviii+812 pp.

[KN1963] KOBAYASHI, S.; NOMIZU, K.m: Foundations of differential geometry, I, Interscience publishers, John Wiley \& Sons, New York, 1963. xi+329 pp.

[Kr1985] KRUZHILIN, N.G.: Local automorphisms and mappings of smooth strictly pseudoconvex hypersurfaces (Russian). Izv. Akad. Nauk SSSR Ser. Mat. 49 (1985), no. 3, 566-591, 672.

[Kr1987] KRUZHILIN, N.G.: Description of the local automorphism groups of real hypersurfaces, Proceedings of the International Congress of Mathematicians, Vol. 1, 2 (Berkeley, Calif., 1986), 749-758, Amer. Math. Soc., Providence, RI, 1987.

[KV1987] KruZhilin, N.G.; Vitushkin, A.G.: Description of the automorphism groups of real hypersurfaces in complex space (Russian). Investigations in the theory of the approximation of functions, 26-69, Akad. Nauk SSSR Bashkir Filial, Otdel. Fiz. Mat., Ufa, 1987.

[Lie1880] LIE, S.: Theorie der Transformationsgruppen, Math. Ann. 16 (1880), 441-528.

[Lie1883] LIE, S.: Klassifikation und Integration von gewöhnlichen Differentialgleichungen zwischen $x$, y, die eine Gruppe von Transformationen gestaten I-IV. In: Gesammelte Abhandlungen, Vol. 5, B.G. Teubner, Leipzig, 1924, pp. 240-310; 362-427, 432-448.

[LS1893] LIE, S.; SCHEFFERS, G.: Vorlesungen b̈er continuierliche Gruppen mit Geometrischen und anderen Anwendungen. (German) Nachdruck der Auflage des Jahres 1893. Chelsea Publishing Co., Bronx, New York, 1971. xii+810 pp.

[Lo1981] LobodA, A.V.: Local automorphisms of real-analytic hypersurfaces (Russian), Izv. Akad. Nauk SSSR Ser. Mat. 45 (1981), no. 3, 620-645.

[Lo2001] LoBODA, A.V.: Homogeneous strictly pseudoconvex hypersurfaces in $\mathbb{C}^{3}$ with twodimensional isotropy groups (Russian) Mat. Sb. 192 (2001), no. 12, 3-24; translation in Sb. Math. 192 (2001), no. 11-12, 1741-1761.

[Lo2002] LobodA, A.V.: Homogeneous nondegenerate surfaces in $\mathbb{C}^{3}$ with two-dimensional isotropy groups (Russian) Mat. Sb. 192 (2001), no. 12, 3-24; translation in Sb. Math. 192 (2001), no. 11-12, 1741-1761.

[Lo2003] LOBODA, A.V.: On the determination of a homogeneous strictly pseudoconvex hypersurface from the coefficients of its normal form (Russian) Mat. Zametki 73 (2003), no. 3, 453-456; translation in Math. Notes 73 (2003), no. 3-4, 419-423.

[Ma2003] MARDARE, S.: On isometric immersions of a Riemannian space under a weak regularity assumption, C. R. Acad. Sci. Paris, Sér. I 337 (2003), 785-790.

[Me2001] MERKER, J.: On the partial algebraicity of holomorphic mappings between two real algebraic sets in the complex euclidean spaces of different dimensions, Bull. Soc. Math. France 129 (2001), no. 4, 547-591.

[Me2003] Merker, J.: hand manuscript I, 212 pp., May-July 2003; hand manuscript II, 114 pp., August 2003.

[Me2004] MERKER, J.: Explicit differential characterization of the Newtonian free particle system in $m \geqslant 2$ dependent variables, Acta Mathematicæ Applicandæ, to appear, 73 pp; e-print: arxiv.org/abs/math.DG/0411165.

[Me2005a] MERKER, J.: On the local geometry of generic submanifolds of $\mathbb{C}^{n}$ and the analytic reflection principle (Part I), Journal of Mathematical Sciences (N.Y.) 125 (2005), no. 6, 751-824.

[Me2005b] MERKER, J.: Étude de la régularité analytique de l'application de réflexion CR formelle, Annales Fac. Sci. Toulouse, XIV (2005), no. 2, 215-330.

[MP2005] MERKER, J.; PORTEN, E.: Holomorphic extension of CR functions, envelopes of holomorphy and removable singularities, $432 \mathrm{pp}$., to appear. Downloadable at: www. cmi . univ-mrs/ merker/index.html.

[N2003] NEUT, S.: Implantation et nouvelles applications de la méthode d'équivalence d'Élie Cartan, Thèse, Université Lille 1, October 2003. 
[OL1979] OLVER, P.J.: Symmetry groups and group invariant solutions of partial differential equations, J. Diff. Geom. 14 (1979), 497-542.

[O11986] Olver, P.J.: Applications of Lie groups to differential equations. Springer Verlag, New York, 1986. xxvi+497 pp.

[O11995] Olver, P.J.: Equivalence, Invariance and Symmetries. Cambridge, Cambridge University Press, 1995, xvi+525 pp.

[OV1994] ONISHCHIK, A.L.; VINBERG, E.B.: Lie groups and Lie algebras, III. Encyclopædia of mathematical sciences, 41. Springer Verlag, Berlin, 248 pp.

[Pi1975] PInchuk, S.: On the analytic continuation of holomorphic mappings (Russian), Mat. Sb. (N.S.) 98(140) (1975) no.3(11), 375-392, 416-435, 495-496.

[Pi1978] PINCHUK, S.: Holomorphic mappings of real-analytic hypersurfaces (Russian), Mat. Sb. (N.S.) 105(147) (1978), no. 4, 574-593, 640; English translation in Math. USSR Sbornik 34 (1978), 503-519.

[Re1993] Reutenauer, C.: Free Lie algebras, London Mathematical Society Monograph, New Series, 7. Oxford Science Publications, The Clarendon Press, Oxford University Press, New York, 1993. xviii+269 pp.

[Se1931] SEgRE, B.: Intorno al problema di Poincaré della rappresentazione pseudoconforme, Rend. Acc. Lincei, VI, Ser. 13 (1931), 676-683.

[Se1932] SEGRE, B.: Questioni geometriche legate colla teoria delle funzioni di due variabili complesse, Rendiconti del Seminario di Matematici di Roma, II, Ser. 7 (1932), no. 2, 59-107.

[Sh1997] ShARPE, R.W.: Differential geometry; Cartan's generalization of Klein's Erlangen program, Graduate texts in mathematics, vol. 166, Springer Verlag, Berlin, 1997, xix+421 pp.

[Sp1970] SPIVAK, M.: A comprehensive introduction to differential geometry, vols. 1 and 2. Published by M. Spivak, Brandeis Univ., Waltham, Mass. 1970.

[St1996] Stanton, N.: Infinitesimal CR automorphisms of real hypersurfaces, Amer. J. Math. 118 (1996), no. 1, 209-233.

[Ste1983] Sternberg, S.: Lectures in differential geometry, Second edition, Chelsea publishing co., New York, 1983, xviii+442 pp.

[Stk1982] Stormark, O.: On the theorem of Frobenius for complex vector fields, Ann. Scuola Norm. Sup. Pisa Cl. Sci. (4) 9 (1982), no. 1, 57-90.

[Stk2000] STORMARK, O.: Lie's structural approach to PDE systems, Encyclopædia of mathematics and its applications, vol. 80, Cambridge University Press, Cambridge, 2000, xv+572 pp.

[Su2001] SukHov, A.: Segre varieties and Lie symmetries, Math. Z. 238 (2001), no. 3, 483-492.

[Su2002] SukHov, A.: CR maps and point Lie transformations, Michigan Math. J. 50 (2002), 369-379.

[Su2003] SukHov, A.: On transformations of analytic structures (Russian). Izv. Ross. Akad. Nauk Ser. Mat. 67 (2003), no. 2, 101-132; transl. in Izv. Math. 67 (2003), no. 2, 303-332.

[Su1973] Sussmann, H.J.: Orbits of families of vector fields and integrability of distributions, Trans. Amer. Math. Soc. 180 (1973), no. 1, 171-188.

[Tr1896] TRESSE, A.: Détermination des invariants ponctuels de l'équation différentielle du second ordre $y^{\prime \prime}=\omega\left(x, y, y^{\prime}\right)$, Hirzel, Leipzig, 1896.

[Ve1924] VeSsIOT, E.: Sur une théorie nouvelle des problèmes généraux d'intégration, Bull. Soc. Math. France 52 (1924), 336-395.

[Vi1990] Vitushin, A.G.: Holomorphic mappings and the Geometry of Hypersurfaces, Encyclopædia of Mathematical Sciences, Volume 7, Several Complex Variables, I, Springer-Verlag, Berlin, 1990, pp. 159-214.

[We1977] WeBSTER, S.M.: On the mapping problem for algebraic real hypersurfaces, Invent. Math. 43 (1977), no.1, 53-68.

CNRS, Université de Provence, LATP, UMr 6632, CMi, 39 Rue Joliot-Curie, F-13453

MARSEILlE CEDEX 13, FRANCE. Internet: http://www. cmi . univ-mrs. fr/ merker/index. html E-mail address: merkerecmi .univ-mrs.fr 